DOE/FE-0360-1

Natural Gas

Imports and Exports

First Quarter Report

1997

Prepared By:

U.S. Department of Energy

Office of Fossil Energy

Office of Natural Gas \& Petroleum Import \& Export Activities

a 51

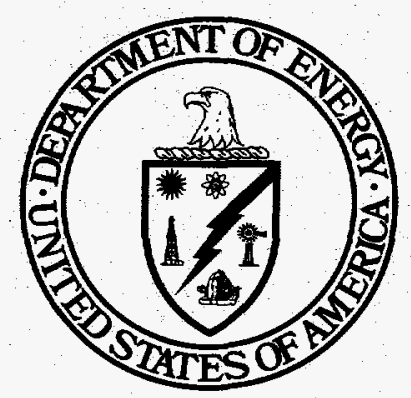




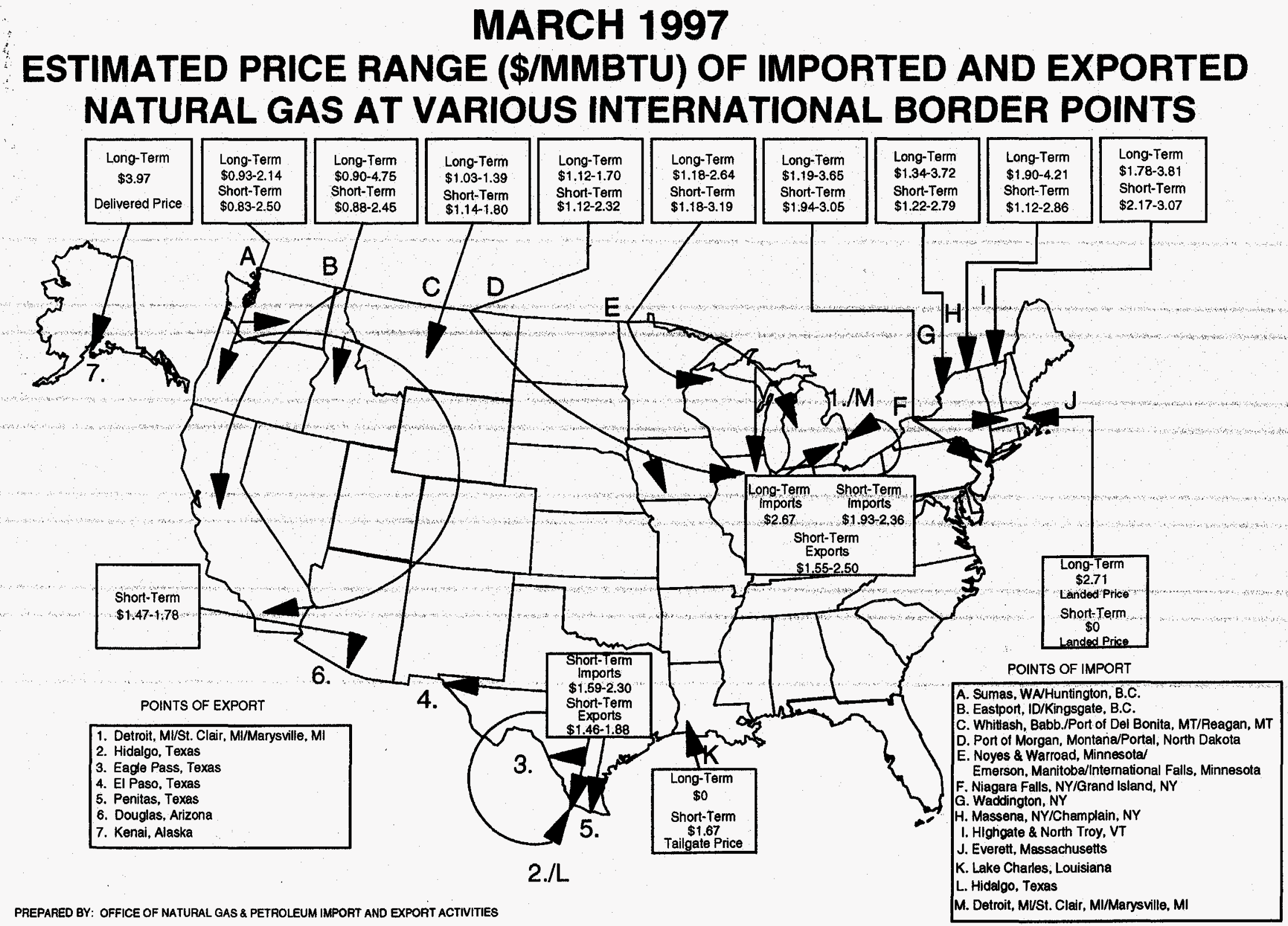




\section{Natural Gas}

Imports and Exports

\section{First Quarter Report \\ 1997}

Prepared By:

\section{U.S. Department of Energy}

Office of Fossil Energy

Office of Natural Gas \& Petroleum

Import \& Export Activities

Washington, DC 20585-0350

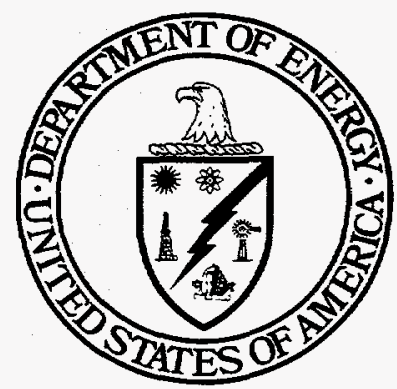




\section{DISCLAMERR}

Portions of this document may be illegible in electronic image products. Images are produced from the best available original document. 
The Office of Natural Gas and Petroleum Import and Export Activities prepares quarterly reports summarizing the data provided by companies authorized to import or export natural gas. Companies are required, as a condition of their authorizations, to file quarterly reports. This report is for the first quarter of 1997 (January through March).

Attachment A shows the percentage of takes to maximum firm contract levels and the weighted average per unit price for each of the long-term importers during the five most recent reporting quarters.

Attachment B shows volumes and prices of gas purchased by long-term importers and exporters during the past 12 months.

Attachment $C$ shows volume and price information pertaining to gas imported on a short-term or spot market basis.

Attachment $D$ shows the gas exported on a shortterm or spot market basis to Canada and Mexico.

First Quarter Highlights: Although the volume of Canadian gas imports declined slightly from its historic high level in the preceding quarter (by 1.7 percent or from 757.3 to $744.7 \mathrm{Bcf}$ ), total imports from all sources set a record high this quarter at $772 \mathrm{Bcf}$, exceeding the record set last quarter (771 Bcf). The highest level of Mexican imports (7 Bcf) since 1984 and the highest level of LNG imports $(20.2 \mathrm{Bcf}$ in the past three years were instrumental in setting this new record. The price of gas exported to Mexico in January reached a record high for the decade at $\$ 4.03$ per MMBtu.

First Quarter Data: Long-term imports for the quarter totaled $372.1 \mathrm{Bcf}$, representing a one percent increase over the first quarter of 1996. Long-term Canadian imports totaled 355.1 Bcf, which is one percent less than the first quarter of 1996. The average price of these supplies was $\$ 2.74$ per MMBtu, which was 20 cents or 8 percent higher than the preceding quarter. Under other long-term import arrangements, Distrigas Corporation imported 12.6 Bcf of Algerian LNG at an average landed price of $\$ 2.85$ per MMBtu and PanEnergy imported 4.4 Bcf of Algerian LNG at $\$ 2.00$ per MMBtu (tailgate price).

During the first quarter, 104 companies used short-term authorizations to import $399.8 \mathrm{Bcf}$ of gas. This volume represents a 7.4 percent increase compared to the short-term imports of the first quarter of 1996 (372.2 Bcf). Of the total imported this quarter, 389.6 Bcf were imported from Canada at an average price of $\$ 2.21$ per MMBtu (compared to \$2.04 per MMBtu in the fourth quarter), and 7 Bcf were imported from Mexico at an average price of $\$ 2.30$ per MMBtu. Under short-term LNG import contracts, Distrigas imported 2.4 Bcf from Abu Dhabi and PanEnergy imported 0.8 Bcf from Algeria.

Approximately 40 percent of the short-term Canadian imports occurred at Eastport, Idaho, at an average price of $\$ 1.71$ per MMBtu; 18 percent at Sumas, Washington, at $\$ 2.07$ per MMBtu; 14 percent at Port of Morgan, Montana, at $\$ 2.33$ per MMBtu; 13 percent at Noyes, Minnesota, at \$2.82 per MMBtu; 5 percent at Niagara Falls, New York, at \$3.31 per MMBtu; 3 percent at Waddington, New York, at \$2.86 per MMBtu; 3 percent at Grand Island, New York, at $\$ 3.62$ per MMBtu; and 4 percent at various other entry points, at $\$ 2.38$ per MMBtu.

In addition, 30 short-term export authorizations were used, exporting a total of 25 Bcf of gas. Thirteen authorizations were used to export 19.5 Bcf to Canada, at an average price of $\$ 2.76$ per MMBtu. Under 16 authorizations, 5.4 Bcf was exported to Mexico at an average price of $\$ 2.80$ per MMBtu. Finally, $0.1 \mathrm{Bcf}$ of LNG was exported to Japan at an average price of $\$ 3.97$ per MMBtu (delivered).

Year to Date Data: Comparing the first quarter of 1996 with this quarter, gas imports increased 4.4 percent or by 32.5 Bcf (772 v. 739.5 Bcf). Canadian imports increased by 2.3 percent $(744.8$ v. $728.4 \mathrm{Bcf}$ ); Mexican imports doubled (7 v. 3.5 Bcf); and LNG imports increased nearly 166 percent (20.2 v. $7.6 \mathrm{Bcf}$ ). During the same time period, total exports declined by 8.6 percent ( 41.7 v. 45.6 Bcf). Exports to Canada decreased by 12.6 percent (19.5 v. $22.3 \mathrm{Bcf}$ ); exports to Mexico decreased by 16.9 percent (5.4 v. $6.5 \mathrm{Bcf})$; and LNG exports remained the same at $16.8 \mathrm{Bcf}$ for both time periods.

This quarter's focus report is " 1996 Natural Gas Import/Export Trade: A Second Look." The quarterly report and any future revisions to the report will be resident on our Electronic Bulletin Board at (202) 586-7853 or on the Fossil Energy Web Site at http:/www.fe.doe.gov. Any questions or comments conceming this report should be directed to Yvonne Caudillo at (202) 586-4587 or by E-mail at wonne.caudillo @hq.doe.gov. 
NATURAL GAS IMPORTS

Volumes and Weighted Average Prices

By Point of Entry and Type of Authorization

(01/01/97 - 03/31/97)

WEIGHTED

AVERAGE

VOLUME

PRICE

POINT OF ENTRY

(MMCE)

Babb, Montana

Champlain, New York

Detroit, Michigan

Eastport, Idaho

Everett, Massachusetts

Grand Island, New York

Highgate Springs, Vermont

International Falls, Minnesota

Lake Charles, Louisiana

Massena, New York

Niagara Falls, New York

North Troy, Vermont

Noyes, Minnesota

Port of Del Bonita, Montana

Port of Morgan, Montana

Sumas, Washington

Waddington, New York

Warroad, Minnesota

Whitlash, Montana

LONG-TERM AUTHORIZATIONS

TOTAL LONG-TERM IMPORTS

$\begin{array}{rl}447.91 & 1.19 \\ 4,180.64 & 3.34 \\ 1,622.58 & 3.35 \\ 56,643.81 & 2.28 \\ 12,618.97 & 2.85^{*} \\ 4,896.57 & 3.52 \\ 2,883.30 & 2.46 \\ 1,932.46 & 2.31 \\ 4,378.48 & 2.00^{* *} \\ 3,118.96 & 3.13 \\ 54,086.90 & 3.04 \\ 2,828.79 & 3.23 \\ 56,300.02 & 2.81 \\ 15.36 & 1.03 \\ 82,025.27 & 2.41 \\ 19,637.28 & 2.44 \\ 62,503.17 & 3.25 \\ 176.32 & 2.40 \\ 1,784.22 & 1.78\end{array}$

$372,081.01$

2.73

\section{SHORT-TERM AUTHORIZATIONS}

Babb, Montana

Champlain, New York

Detroit, Michigan

Eastport, Idaho

Everett, Massachusetts

Grand Island, New York

Hidalgo, Texas

Highgate Springs, Vermont

Lake Charles, Louisiana

Massena, New York

Niagara Falls, New York

Noyes, Minnesota

Port of Morgan, Montana

Portal, North Dakota

St. Clair, Michigan

Sumas, Washington

Waddington, New York

Warroad, Minnesota

$\begin{array}{rl}5,933.25 & 1.32 \\ 320.05 & 2.78 \\ 1,862.22 & 2.82 \\ 153,831.67 & 1.71 \\ 2,416.67 & 3.35^{*} \\ 12,289.95 & 3.62 \\ 6,959.26 & 2.30 \\ 267.37 & 2.41 \\ 761.54 & 2.85^{* *} \\ 1,012.59 & 2.77 \\ 20,935.66 & 3.31 \\ 51,116.02 & 2.82 \\ 54,885.42 & 2.33 \\ 1,258.78 & 2.20 \\ 4,004.76 & 3.68 \\ 68,814.31 & 2.07 \\ 13,034.00 & 2.86 \\ 49.25 & 2.42 \\ & \\ 399,752.76 & 2.22\end{array}$

\section{TOTAL SHORT-TERM IMPORTS}

$399,752.76$

2.22

* Average landed price

** Average tailgate price

Note: Import figures in this table may vary slightly from sum of components in report due to independent rounding in calculations. 


\section{QUARTERLY FOCUS}

\section{NATURAL GAS IMPORT/EXPORT TRADE: A SECOND LOOK}

The Focus feature that was included in the fourth quarter 1996 Quarterly Report of Natural Gas Imports and Exports ("Report") provided an overview of natural gas import/export activity for calendar 1996. This Focus feature, like the one found in the Report issued for the first quarter of 1996, provides more detailed information pertaining to 1996 cross-border natural gas pipeline trade between the United States, Canada and Mexico. Specifically, this Focus provides additional information on market shares, prices, specific identities of major importers and exporters, as well as updated information on Canadian natural gas sales to its largest state market, California.

\section{Natural Gas Trade with Canada}

During 1996, there were a total of 201 importers of Canadian natural gas; this is an increase of ten over last year's figure of 191 . These 201 importers brought into the United States a record volume of 2,883 billion cubic feet (Bcf) of natural gas during the year. This represented an increase of $67 \mathrm{Bcf}$, or a 2.4 percent over the 1995 total of $2,816 \mathrm{Bcf}$. Canadian natural gas exports to the United States have increased every year over the past decade (1987 - 1996). Although Canadian gas supplies were competitively priced and demand for natural gas continued to increase in the United States during 1996, the rate of growth for Canadian gas imports was the lowest in ten years. The increase in Canadian gas imports abated somewhat in 1996 due in large part to the lack of sufficient pipeline capacity into the United States. Notwithstanding pipeline constraints, the greatest areas of growth for Canadian gas supplies in 1996 occurred in the Pacific Northwest and the Mid-Atlantic States.

With regard to U.S. natural gas exports to Canada during 1996, there were a total of 21 firms making sales of $61.4 \mathrm{Bcf}$ of natural gas to Canada at an average price of $\$ 2.78$ per MMBtu. Exports to
Canada in 1996 increased by 110 percent over the 1995 level of $29.2 \mathrm{Bcf}$. All of the natural gas export transactions were accomplished under the Department of Energy's (DOE) two-year "blanket" export authorizations (for gas sales contracts of two years or less). Like previous years, most of the natural gas exports to Canada occurred at the Michigan exit points of Detroit (30.4 Bcf) and St. Clair (19.3 Bcf). These two exit points accounted for 81 percent of all natural gas exports to Canada during the year. [The 1996 natural gas exports of 61.3 Bcf reflect exports to Canada on an equity (sales) basis rather than on a custody (physical movements) basis; total gas exports on a custody only basis equaled $51.9 \mathrm{Bcf}$ for the year.]

The weighted average international border price of Canadian natural gas imported into the United States during 1996 was $\$ 1.92$ per million British thermal unit (MMBtu). This represented an increase of 32 percent over last year's average price of $\$ 1.45$ per MMBtu. However, putting these figures into perspective, last year's prices were at a record low and the 1994 average price for Canadian gas supplies was $\$ 1.80$ per MMBtu. Under DOE's two-year "blanket" import authorizations (for gas purchase contracts of two years or less), the average border price of gas supplies imported from Canada in 1996 was $\$ 1.63$ per MMBtu. This price represented an increase of over 40 percent over last year's average price of $\$ 1.16$ per MMBtu for short-term Canadian imports. Under DOE's long-term import authorizations (for gas purchase contracts longer than two years), the average border price was $\$ 2.21$ per MMBtu, or an increase of 26 percent over last year's average price of $\$ 1.75$ per MMBtu.

Of the 2,883 Bcf of Canadian gas imported in 1996, 50.3 percent $(1,451 \mathrm{Bcf})$ was imported under DOE's short-term import authority, while 49.7 percent $(1,432 \mathrm{Bcf})$ was imported under its long-term authority. 1996 represented the second straight year in which more Canadian natural gas was imported 
under DOE's short-term import authorizations than under its long-term import authorizations. Comparing 1996 Canadian imports with 1995 imports by type of DOE authorization used, there virtually was no change. Figure 1 below illustrates the steady growth in the use of short-term import authorizations over the past 12 years (1985-1996). Canadian gas imports under long-term authorizations have remained relatively stable over the past three years, thanks largely to the growth in long-term sales to the non-utility generation sector (NUGS). For example, in 1996 nine new long-term supply contracts serving the NUGS were activated; the volumes imported under these contracts represented almost half of the incremental growth in long-term Canadian imports during the year.

Much of the growth in the use of short-term import authorizations as shown in Figure 1, particularly in 1994 and 1995, is due to changes that occurred in the way Canadian gas is marketed in the state of
California, the largest state market for Canadian gas. In 1996, almost 23 percent of all Canadian natural gas exported to the United States was marketed in California. New policies and regulations implemented by the Califomia Public Utilities Commission (CPUC) and the Federal Energy Regulatory Commission (FERC) during the early 1990 's, and the addition of new pipeline capacity serving the State, promoted a more open and competitive gas marketplace. With the implementation of FERC Order 636 and the start-up of the joint pipeline expansion project of Pacific Gas \& Electric Company (PG\&E) and Pacific Gas Transmission Company (PGT) on November 1, 1993, PGT, the largest importer of Canadian natural gas for over 30 years ceased being an importer and became a transportation-only pipeline. The termination of PGT's long-term gas supply contract resulted in the Canadian gas being sold primarily under short-term contracts rather than under longterm contracts.

Figure 1

\section{CANADIAN NATURAL GAS IMPORTS BY TYPE OF IMPORT AUTHORIZATION $1985-1996$}

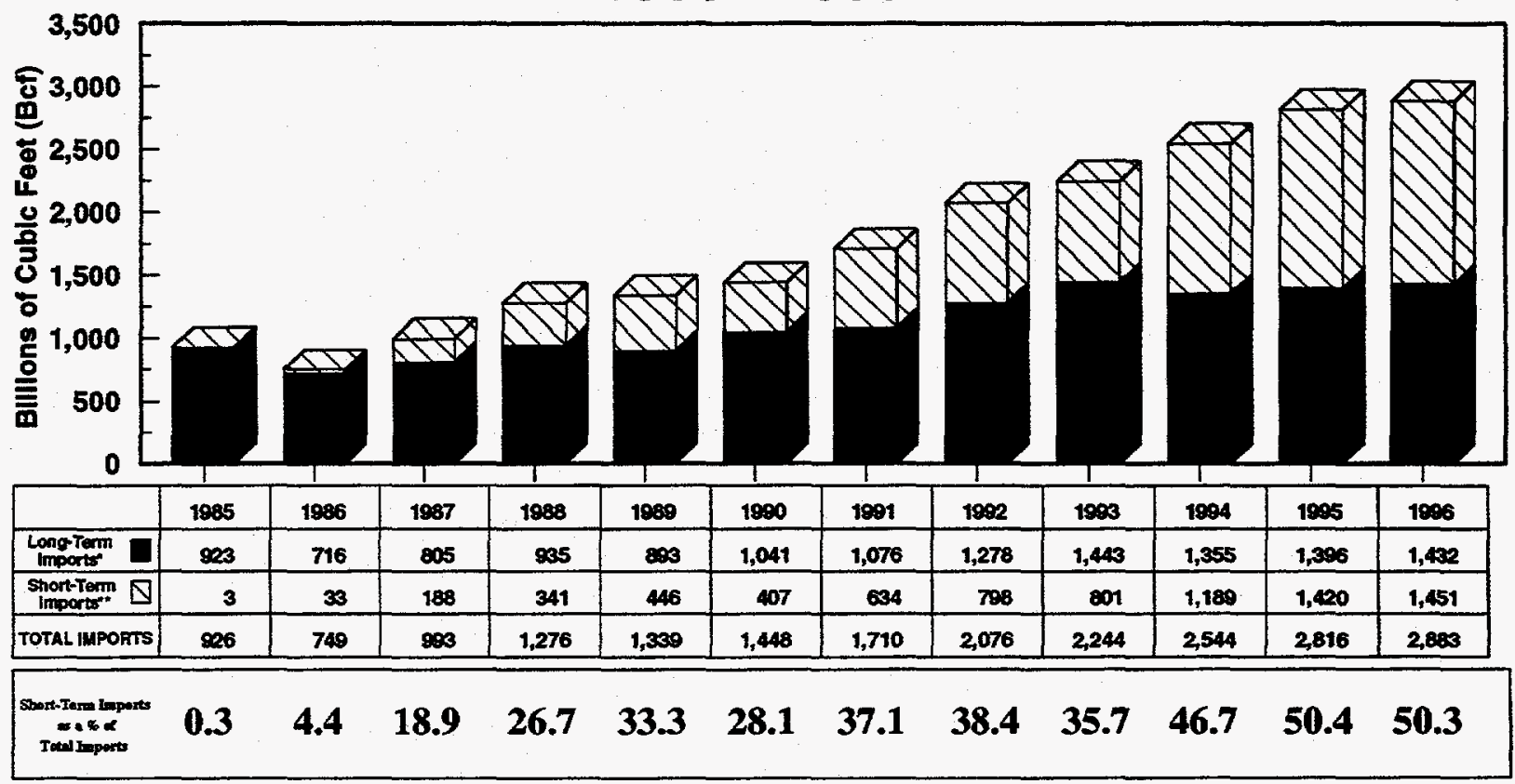

- Imports made under gas purchase contracts longer than 2 years.

* Imports made under gas purchase contracts which are 2 years or less. 
Figure 2 shows the volume of Canadian natural gas marketed in California under both short-term and long-term contracts during the past ten years (1987 1996). As noted above, prior to November 1993, most Canadian gas sales were sold in California under long-term contracts; subsequently, most Canadian gas sales have been made under short-term contracts. During 1996, 659.4 Bcf of Canadian natural gas was marketed in California. Almost 77 percent of the volume marketed in the state during the year was purchased under short-term contracts at an average international border price of $\$ 1.13$ per MMBtu. The remaining 23 percent of the Canadian gas volumes was marketed under long-term contracts at an average international border price of $\$ 1.25$ per MMBtu.

Based on preliminary figures published by the Energy Information Administration (EIA) in its Natural Gas Monthly [DOE/EIA-130 (April 1997), Tables 14-18], total natural gas deliveries to Califomia in 1996 declined by $132.5 \mathrm{Bcf}$, or 7.2 percent from the 1995 level (1707.2 v. $1839.7 \mathrm{Bcf}$ ). The 1996 decline in natural gas deliveries in the state of Califomia follows on the heels of a significant decline in 1995 compared with the 1994 level. Over the past two years, natural gas deliveries in Califomia have plunged $334.3 \mathrm{Bcf}$, or 16.4 percent.
Approximately 85 percent of the overall drop in natural gas deliveries during the past two years was directly the result of reduced demand for natural gas in the electric utility sector. Natural gas deliveries to electric utilities declined 34 and 19 percent in 1995 and 1996, respectively. This large decline in natural gas use in the electric utility sector was the result of power plants in California relying on the increased availability of less expensive hydroelectric generation due to the unusually heavy rains and snows in the Pacific Northwest and California over the past couple of years. Additional reductions in gas demand in this sector also may have been caused by the removal from service of smaller and older fossil plants by certain California utilities.

Consistent with the general decline in natural gas deliveries in Califomia during 1996, Canadian natural gas supplies marketed in California, as shown in Figure 2, also dropped by $55.3 \mathrm{Bcf}$, or 7.7 percent from the 1995 level. On a percentage basis, Canadian long-term and short-term imports declined by about the same amount. Long-term imports declined by 7.8 percent in 1996 and short-term imports declined by 7.6 percent. Short-term imports declined from 548.3 Bcf in 1995 to 505.7 Bcf in 1996, while long-term imports declined from 166.4 Bcf in 1995 to 153.7 Bcf in 1996.

Figure 2

CANADIAN NATURAL GAS EXPORTS TO CALIFORNIA UNDER LONG-TERM AND SHORT-TERM CONTRACTS OVER THE PAST TEN YEARS: 1987 - 1996 (MMCF)

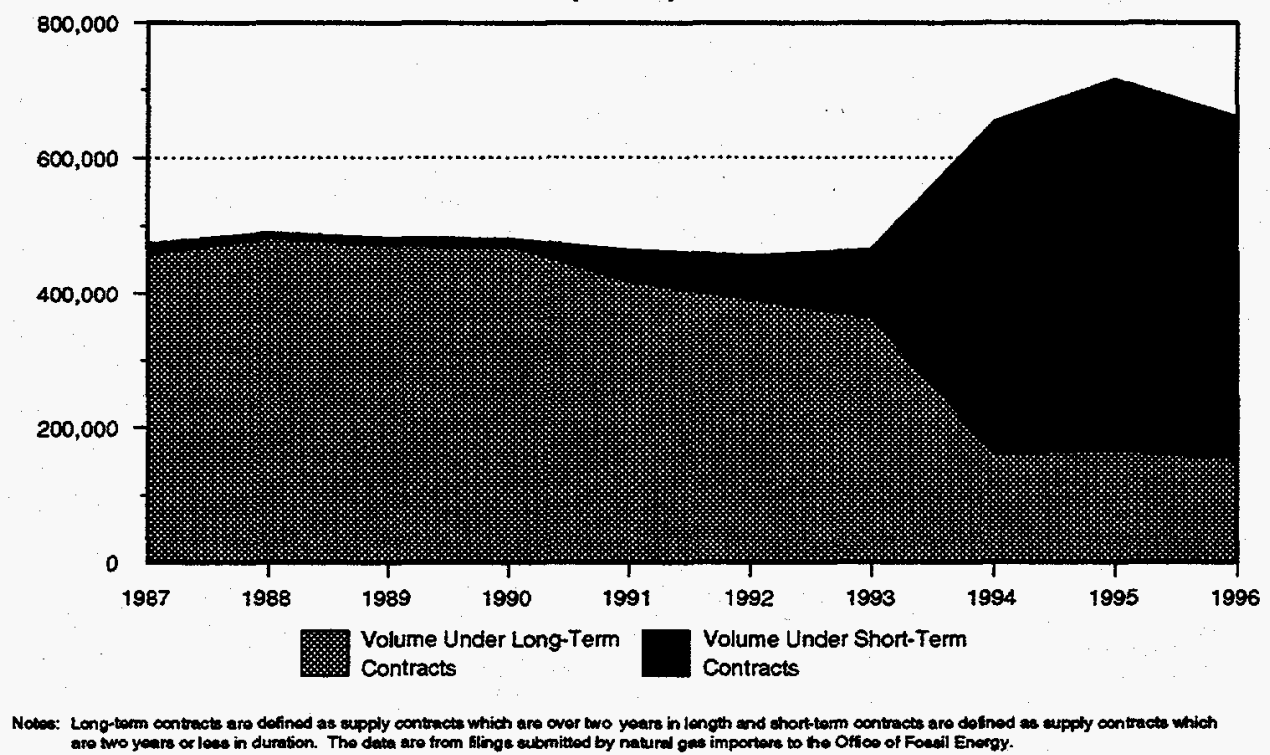

North American Gas Trade * North American Gas Trade * North American Gas Trade * North American Gas Trade * North American Gas Trade 
Figure 3 shows Canadian natural gas marketed in California as a percentage of total gas consumption for the state during the past ten years (1987 - 1996). This figure merely displays the growth in market shares for Canadian gas in California during this time period. With the advent of a more competitive marketplace and increased pipeline capacity serving the state, the market share for Canadian gas has grown from 24.6 percent in 1987 to approximately 37 percent in 1995 and 1996.

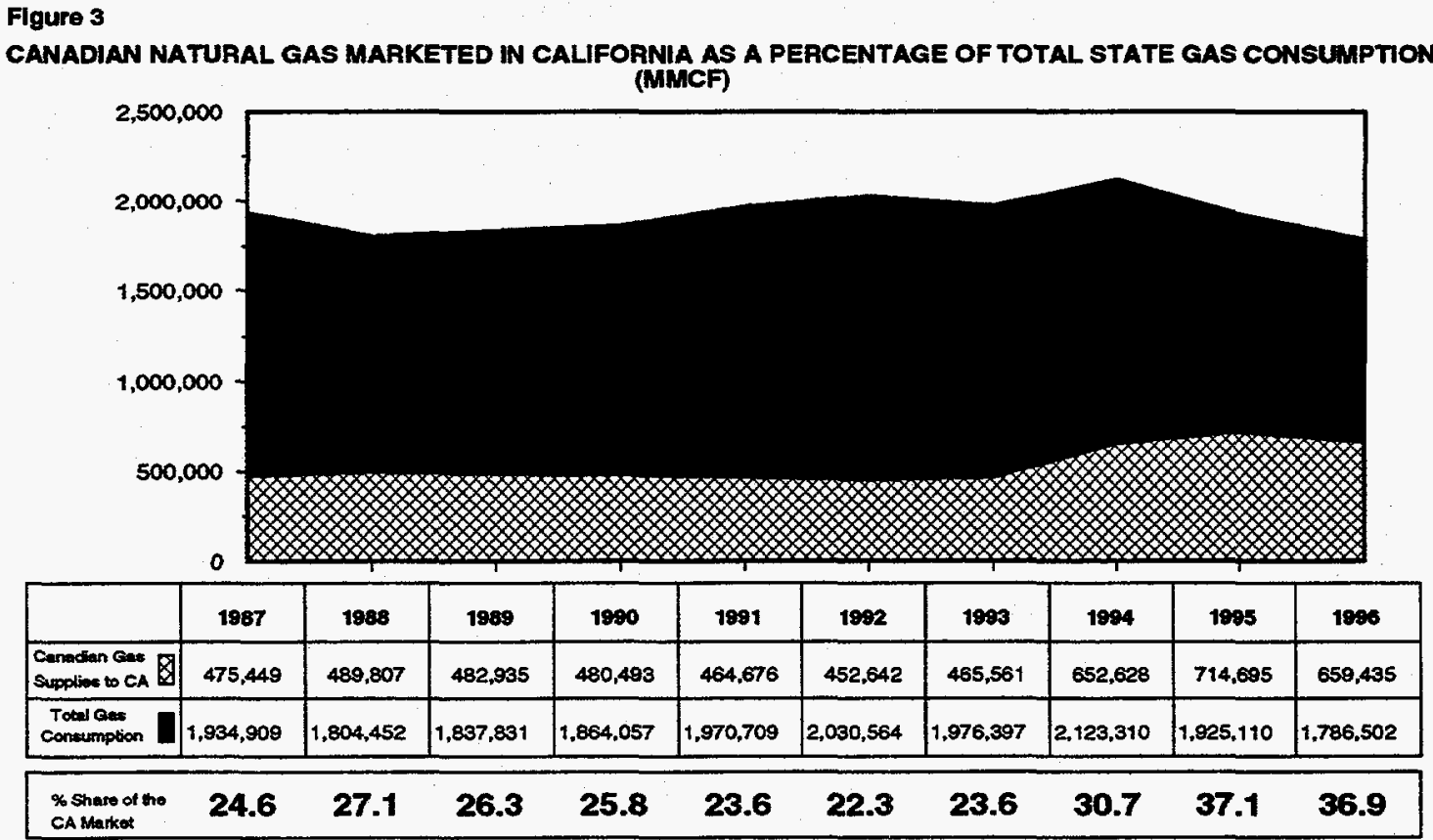

Sources: Consumption data for 1987 thru 1995 obtained from the Natural Cas Annual (DOEfElA-0131); 1996 consumption figure is an FE estimate based on preliminary ElA data. Canadian natural gas supplies marketed in Califomia are from reports filed by importers with FE.

Figure 4 lists the top ten importers of Canadian natural gas for the year. These ten firms imported a total of $1193.9 \mathrm{Bcf}$ of natural gas, or over 41 percent of the total Canadian gas imported for the year. Figure 4 also indicates whether the imports were made under short-term or long-term import authorizations. About 55 percent of the volumes imported by this group of importers was done under DOE's short-term import authority and 45 percent was under long-term import authority. Eight out of the top ten Canadian natural gas imports listed in 1995 were also among the top ten listed for 1996. The only year-to-year changes consisted of Coastal Gas Marketing Company and Washington Natural Gas Company replacing Mobil Natural Gas Inc., and Enron Capital \& Trade Resources Corporation from the list. Among the top importers in 1996, there were only three end-users: a local gas distributor, a combined electric/gas utility and an electric utility; the rest of the importers were marketers, producer affiliates, or gas aggregators.
Figure 4

TEN LARGEST IMPORTERS OF CANADIAN NATURAL GAS IN 1996

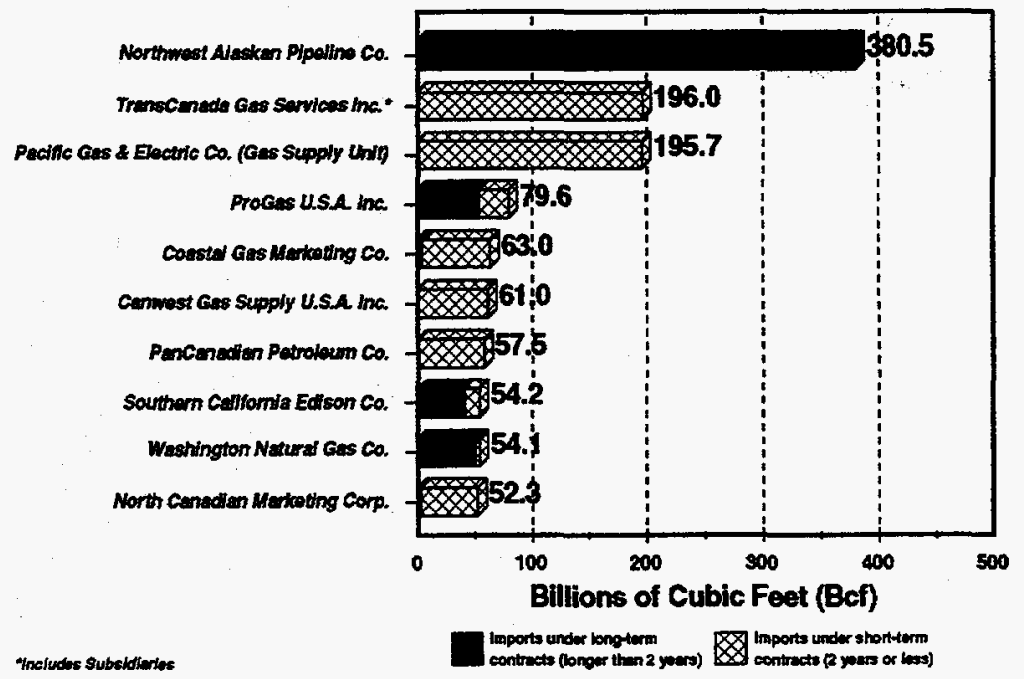


Figure 5 lists the ten largest suppliers of Canadian natural gas to the United States in 1996. The volumes supplied by each company include both short-term and long-term sales. Eight out of ten of these suppliers were also on the list of top gas suppliers for 1995; however, Pan Energy Marketing Canada and PanCanadian Petroleum Ltd., replaced Enron Capital \& Trade Resources Canada and Mobil Natural Gas Canada in 1996. This list of gas suppliers also does not reflect some recent marketing alliances and mergers of certain companies, e.g., Coastal and Westcoast Gas. Most of these marketing alliances occurred in late 1996, or the early part of 1997. As shown, most of the top suppliers of Canadian natural gas to the United States in 1996 were gas aggregators. The top ten suppliers of Canadian natural gas listed in Figure 5 supplied slightly over 67 percent of all Canadian gas imports during 1996 (1938.7 Bcf); in comparison, during 1995 the ten largest suppliers marketed almost 64 percent of all Canadian gas going to the United States.

The next three graphs provide information pertaining to Canadian natural gas imports made in 1996 under long-term import authorizations. Figure 6 indicates what type of importer was purchasing Canadian natural gas under long-term purchase contracts. The figure shows, by type of importer, the actual volumes imported and percentage of market share. Like 1995, local gas distribution companies (LDCs) and natural gas marketers continue to import the largest shares of Canadian natural gas under longterm contracts, 39.4 percent and 35.9 percent, respectively. For the most part, the volumes being imported today by LDCs and marketers have replaced those volumes imported by interstate pipelines in the past. Comparing the market share breakdown in 1996 with 1995, market shares in 1996 increased for LDCs, marketers, non-utility generation sector (NUGS), and municipalities/industrial customers, but declined for electric utilities and interstate pipelines. On a percentage basis, both marketers and NUGS showed over an 8 percent increase in the volumes they imported in 1996 over their 1995 levels.

Figure 7 on the following page illustrates the differences in average commodity prices paid by the various types of long-term importer for 1995 and
Figure 5

TEN LARGEST SUPPLIERS OF CANADIAN NATURAL GAS IN 1996

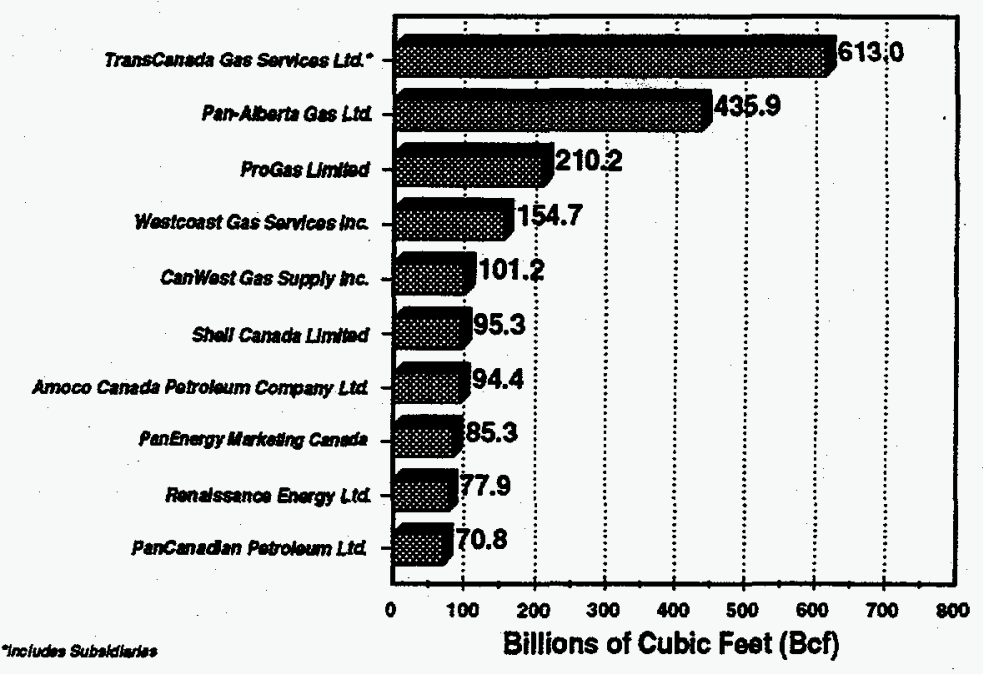

Figure 61996 CANADIAN NATURAL GAS IMPORTS UNDER LONG-TERM IMPORT AUTHORIZATIONS BY TYPE OF IMPORTER

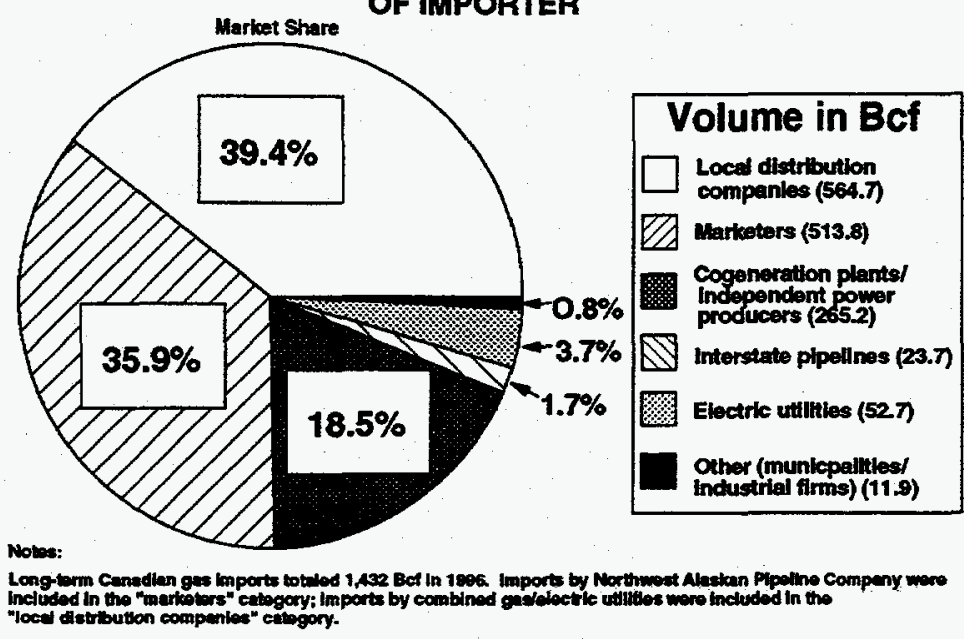

1996. In 1996 , the average commodity price paid by all importers of Canadian natural gas under longterm contracts was $\$ 1.70$ per MMBtu. This price is $\$ 0.50 / \mathrm{MMBtu}$, or 41.7 percent higher than last year's average price of $\$ 1.20$ per MMBtu. The average commodity price increase shown for all Canadian long-term imports tracks closely with the increases experienced in 1996 for average domestic wellhead prices. In 1996, natural gas wellhead 
prices rose to $\$ 2.25$ per thousand cubic feet (Mcf). The EIA indicated that the 1996 average wellhead price was 45 percent greater than the $\$ 1.55$ recorded in 1995 and, after adjustment for inflation, was the highest level since 1986 [DOE/EIA-130, Natural Gas Monthly, May 1997, Table 4, p.12].

Figure 7 shows there were some differences in commodity prices paid among the various types of importer in 1996, but the price differences were not nearly as large as those experienced in 1995. Among the four principal types of importer, only the LDCs, on average, paid less than the aggregate average price paid for the year, while interstate pipelines and NUGS paid more than the aggregate average price. Although the LDCs paid, on average, the lowest commodity price during 1996 ( $\$ 1.67 / \mathrm{MMBtu})$, this category of importer had the greatest year-to-year price increase. The 1996 LDC average commodity price represented a 79.6 percent increase over the 1995 average price of $\$ 0.93$ per MMBtu. The category of importer which experienced the next largest jump in prices over the 1995 level was marketers. Most of the underlying long-term supply contracts for both the LDCs and marketers have great price elasticity because they track closely to changes in the natural gas and oil markets. Therefore, the prices under these contracts dropped dramatically during 1995 when oil and gas prices fell, but when the prices of oil and gas climbed in 1996, the price under these contracts followed suit. As shown in Figure 7, the NUGS category of importer showed the smallest increase in commodity prices in 1996 compared with 1995 . The average commodity price of NUGS went from $\$ 1.55$ per MMBtu in 1995 to $\$ 1.77$ per MMBtu in 1996, or an annual increase of 14.2 percent. The principal reason why the commodity price for NUGS did not escalate nearly as fast as other importer groups is that most of the underlying contracts are not directly pegged to changes in the oil and natural gas marketplace. Many of the long-term gas supply contracts used by NUGS have fixed annual price adjustments; therefore, many of these contracts were not impacted by the price swings in the oil and gas marketplace experienced in 1996. The average commodity price of $\$ 1.77$ per MMBut paid by the NUGS in 1996 was 4.1 percent above the average aggregate price of $\$ 1.70$ per MMBtu paid by all importers, while the average price paid by LDCs was 1.8 percent less than the aggregate average price.

Figure 7

THE WEIGHTED AVERAGE COMMODITY PRICE IN 1995 AND 1996 FOR CANADIAN NATURAL GAS IMPORTED UNDER LONG-TERM CONTRACTS BY TYPE OF IMPORTER (\$MMBtU)

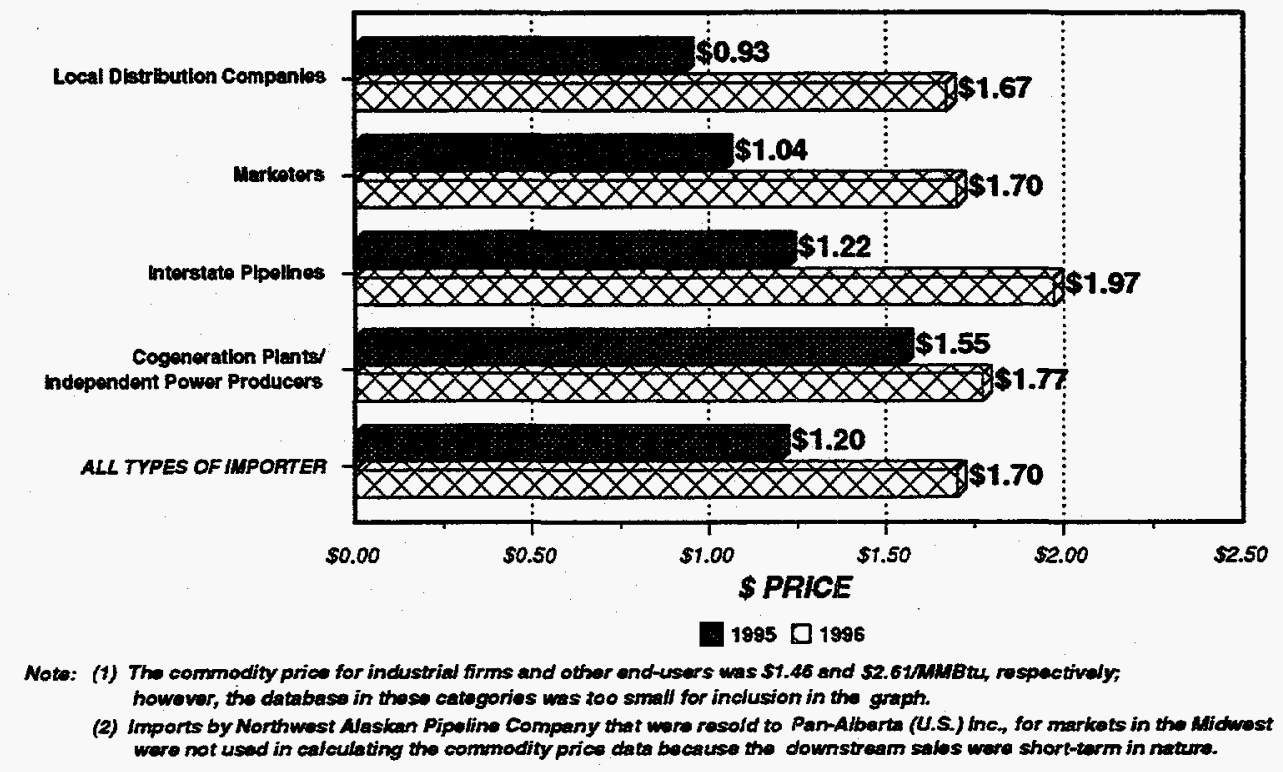

North American Gas Trade * North American Gas Trade * North American Gas Trade * North American Gas Trade * North American Gas Trade 
Figure 8 shows the different commodity prices, by Census Region, paid for Canadian natural gas imported under long-term contracts for both 1995 and 1996. It shows that the large differences in commodity prices among the various Census Regions which occurred in 1995 have not disappeared during 1996. In 1996, the areas of the country which continue to experience the least expensive commodity prices are in Census Regions 8 and 9, which cover the Western and Mountain States, with prices ranging from $\$ 0.97$ to $\$ 1.12$ per MMBtu. However, unlike 1995, this year's commodity prices in the Census Regions representing the rest of the country were found to fall into a fairly close price range. The only exception to this trend is Census Region 5 (South Atlantic); the relatively high average commodity price in this region is influenced by a small database and a distressed NUGS contract. During 1996, the average commodity prices in the Midwestem states (Census Regions 3 \& 4) rose marginally higher than those in the New England states (Census Region 1). The New England States average commodity price was favorably affected by NUGS contracts in 1996 as their commodity costs, on average, did not increase as fast as other types of long-term importers.

There continues to be a large difference in Canadian natural gas commodity costs among various parts of the country, as illustrated in Figure 8. However, the price dichotomy seems to have gone from a split between the West Coast and the East Coast in 1995 to more of a price split between the West Coast and the rest of the country in 1996. The relatively small variances in commodity prices among long-term importers in the Midwestern and Eastern states seem more of a function of the type of importer, or contract performance in the regions, rather than the geographical region served. The factors which contributed to less expensive commodity prices in the Western and Mountain States in 1995 went basically unchanged in 1996 . First, there currently exists a large surplus of natural gas production in both western Canada and western United States competing for the same western markets, with limited access to Midwestern and Eastern markets. Second, there continues to be a large surplus of natural gas pipeline capacity serving the western markets, particularly to California. Third, the pipeline transportation system used to deliver Canadian natural gas to the California market in Census Region 9 is relatively expensive compared with costs associated with delivering southwest gas to the same market; therefore, the commodity costs must remain lower in order to keep Canadian natural gas competitive in this market. Fourth, competition in the western markets continues to be particularly intense in light of the surplus indigenous supplies and the fact that for the second consecutive year, California experienced a substantial drop in natural gas demand, primarily caused by surplus hydro-electric generation capacity.

\section{Figure 8}

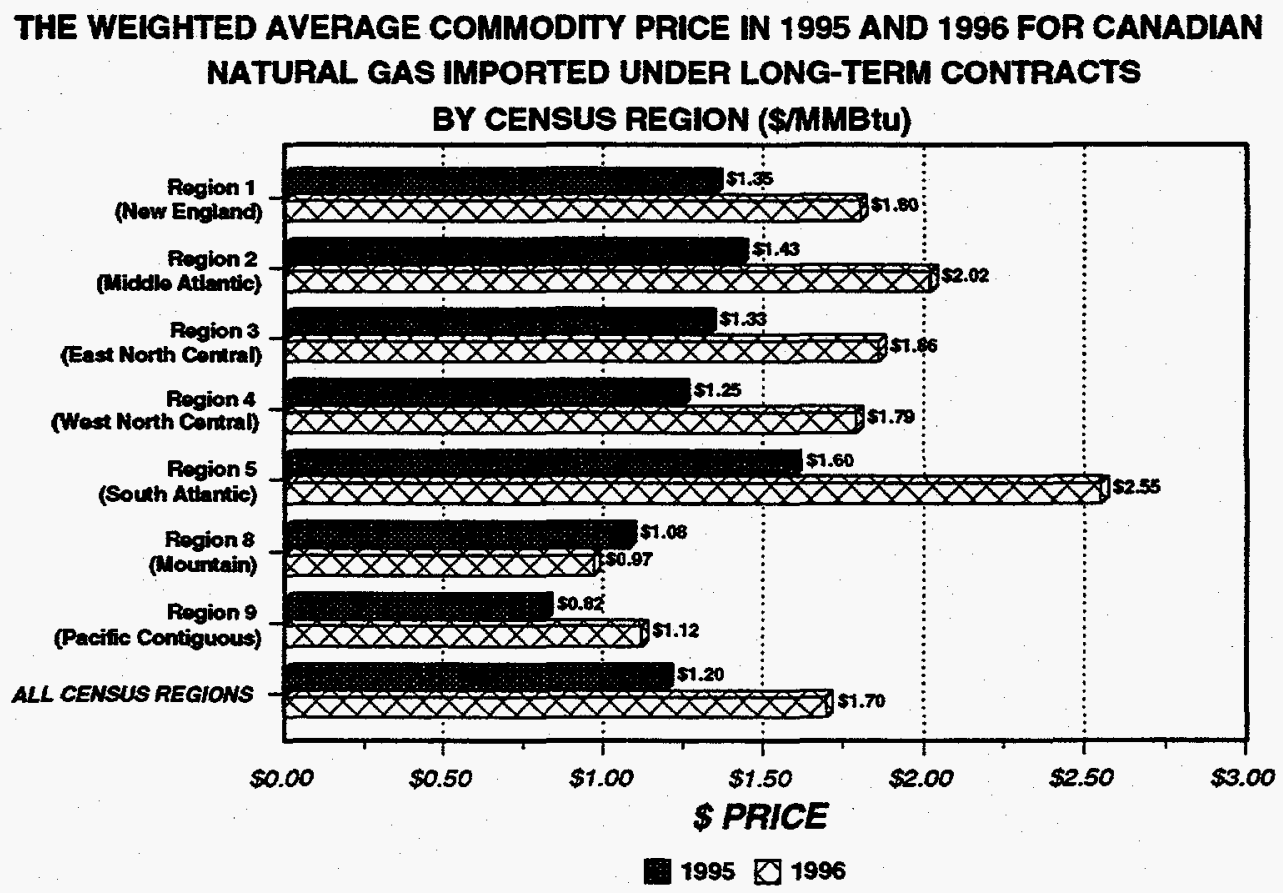

North American Gas Trade * North American Gas Trade * North American Gas Trade * North American Gas Trade * North American Gas Trade 
The next two graphs provide information on Canadian natural gas imported under DOE's shortterm blanket authorizations during 1996. As mentioned earlier, Canadian natural gas imports under this type of import authority have exceeded the volume imported under long-term authority for both 1995 and 1996. Figure 9 identifies, by class of importer, the market share of those who imported Canadian natural gas in 1996 under short-term import authorizations. As displayed in Figure 9, there were three principal types of short-term importer: marketers, LDCs, and Canadian gas producers or their U.S. affiliates. These three types of importer brought in almost 97 percent of the total short-term Canadian natural gas imports in 1996. Comparing the market share breakdown in 1996 with 1995, marketers and industrial firms were the only two categories of short-term importer that experienced growth in market shares during the past year.

Figure 10 shows for 1995 and 1996 the weighted average international border price for Canadian natural gas imported under short-term contracts by Census Region. Almost 99 percent of all short-term Canadian natural gas sales to the United States in 1996 were concentrated in five Census Regions $(2,3,4,8,9)$, with over 53 percent of the volumes marketed in Census Region 9 (Pacific Contiguous). As indicated, the average border price for all shortterm imports in 1996 was $\$ 1.63$ per MMBtu, compared with $\$ 1.16$ per MMBtu in 1995 ; this constitutes an increase of 40.5 percent. There were three Census Regions which showed year-to-year price changes that were below the national average of 40.5 percent: eight (15.7\%), nine ( $25.5 \%)$, and one
(38.9\%). It's easy to understand why Census Regions 8 (Mountain States) and 9 (Pacific Contiguous States) experienced less than average gas price increases in 1996 due to the indigenous gas supplies and surplus transportation capacity serving these regions, but the lower than average price increase in Census Region 1 (New England States) is less apparent. Although short-term Canadian gas sales to New England in 1996 remained relatively small $(13.1 \mathrm{Bcf})$, the lower than average price increases probably were the result of purchases by the NUGS and increased competition from alternative energy sources, including the increased availability of liquefied natural gas imports.

During 1996, the national average price of $\$ 1.63$ per MMBtu for short-term imports was $\$ 0.07$ per MMBtu less than the average commodity price paid for imports under long-term contracts (see Fig. 7). In 1995, the price differential between the average price for short-term imports and the average commodity price under long-term contracts was $\$ 0.04$ per MMBtu. The fact that the average commodity price for long-term contracts tracks so closely to the price under short-term contracts is indicative of how closely the pricing provisions of long-term sales arrangements are tied to the price fluctuations in the marketplace. Figure 10 also illustrates the price split among the various regions. Like the long-term import prices, as previously shown in Figure 8, there is a considerable price dichotomy between the natural gas prices in the Westem states (Census Regions 8 \& 9) and the rest of the country. Census Region 5 (South Atlantic) showed the highest average natural gas price Canadian supplies during 1996, but the price is skewed due to the small volume of Canadian natural gas (less than $1 \mathrm{Bcf}$ ) sold in this region. 
Figure 9

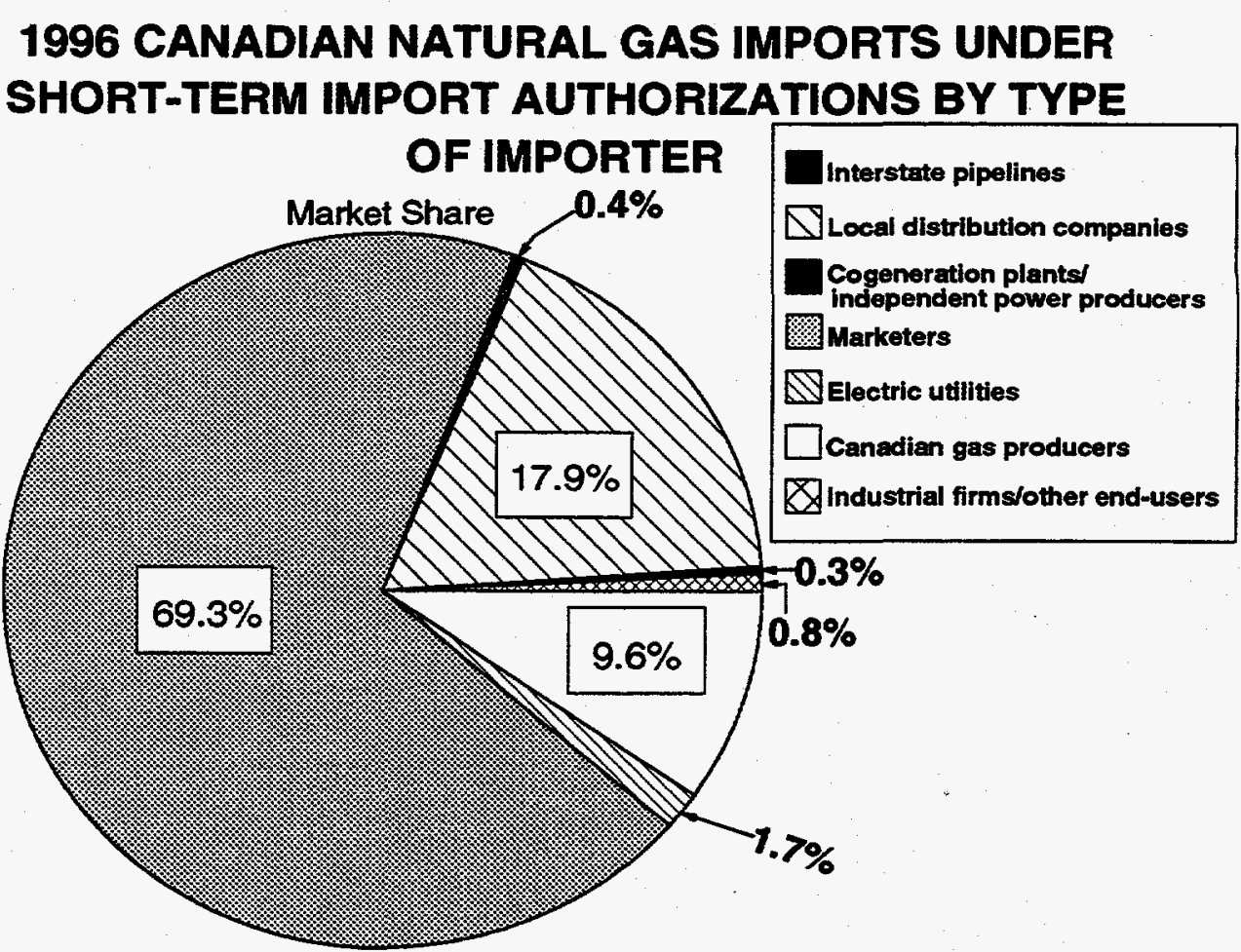

Total short-term imports: 1,451 Bef

Figure 10 THE WEIGHTED AVERAGE PRICE IN 1995 \& 1996 FOR CANADIAN NATURAL GAS IMPORTED UNDER SHORT-TERM CONTRACTS BY CENSUS REGION (\$MMBtu)

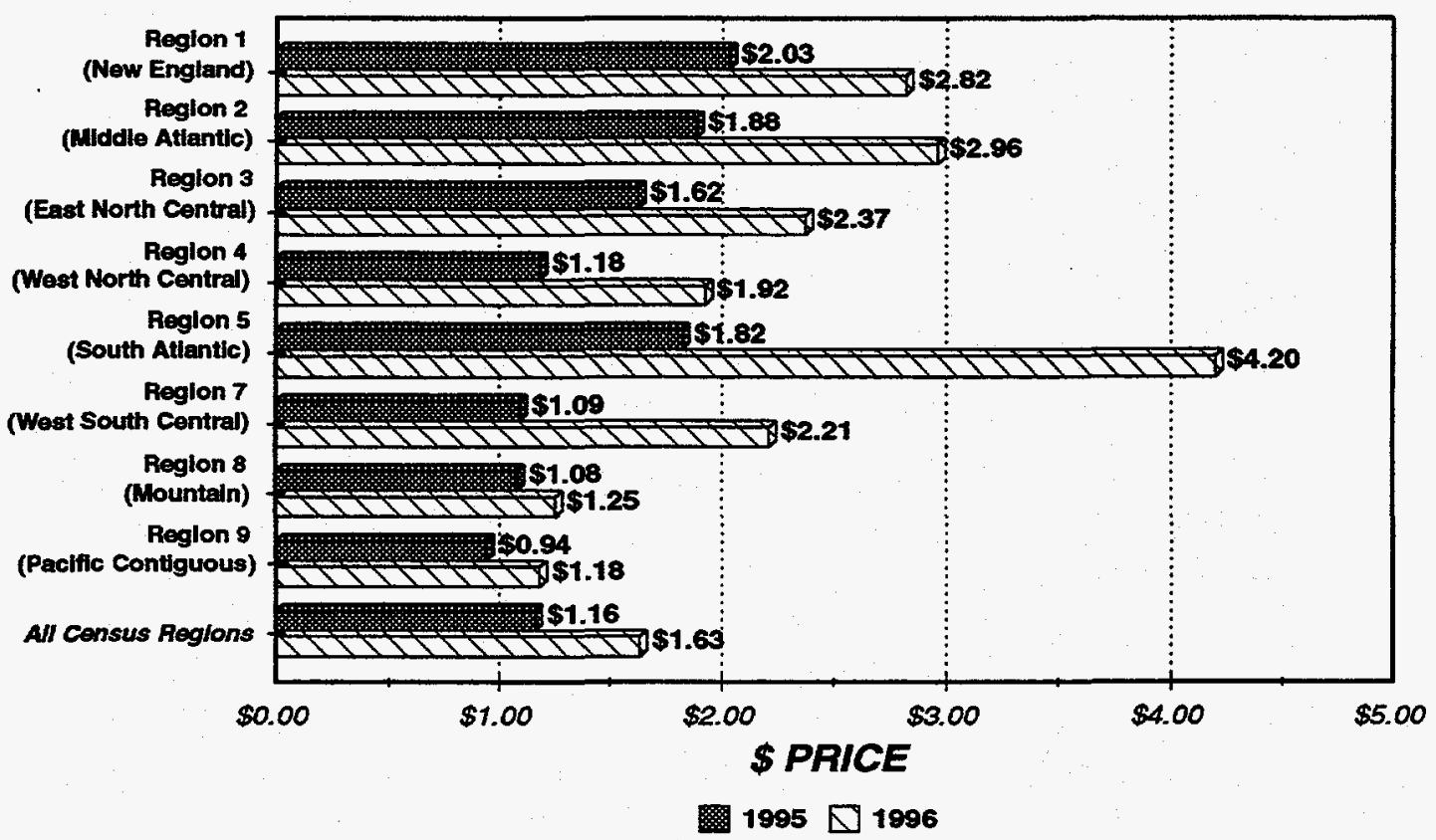

North American Gas Trade * North American Gas Trade * North American Gas Trade * North American Gas Trade * North American Gas Trade 


\section{Mexican Gas Trade}

The last four graphs provide information on Mexican gas trade during 1996. Figure 11 identifies the 29 firms that exported a total of $33.8 \mathrm{Bcf}$ of natural gas to Mexico in 1996, and indicates the market share of the six largest exporters. There were a record number of companies exporting natural gas to Mexico in 1996, an increase of 8 over last year's figure of 21 exporters; however, total volumes for 1996 declined by $27.5 \mathrm{Bcf}$, or 45 percent from the 1995 level. Amoco Energy Trading Corporation continued to be the largest exporter of natural gas to Mexico; however, its market share in 1996 represented only 18 percent of total export volumes as compared to 33 percent in 1995. Although there were a total of 29 exporters in 1996, Amoco and the other top five gas exporters represented about 64 percent of the Mexican import market.

Figure 12 shows the 20 companies that imported a total of 13.9 Bcf of Mexican natural gas into the United States, more than double the 1995 level $(6.7 \mathrm{Bcf})$. The majority of gas (13.6 Bcf) entered the U.S. at Hidalgo, Texas, while the remaining volumes $(.3 \mathrm{Bcf})$ entered the country at Penitas, Texas. The number of companies importing Mexican natural gas this year increased by 8 from last year's figure of 12 importers. Mexican sources predict that exports to the United States will continue to grow in 1997, with total volumes expected to approach the figure for Mexican gas imports from the U.S.

Figures 13 and 14 provide monthly volume and price information with regard to natural gas exports to Mexico over the past five years (January 1992 - December 1996). The monthly weighted average price of natural gas exports to Mexico was at its highest this decade in December 1996, when the price climbed to $\$ 3.68$ per MMBtu.
FIRMS THAT EXPORTED NATURAL GAS TO MEXICO IN 1996

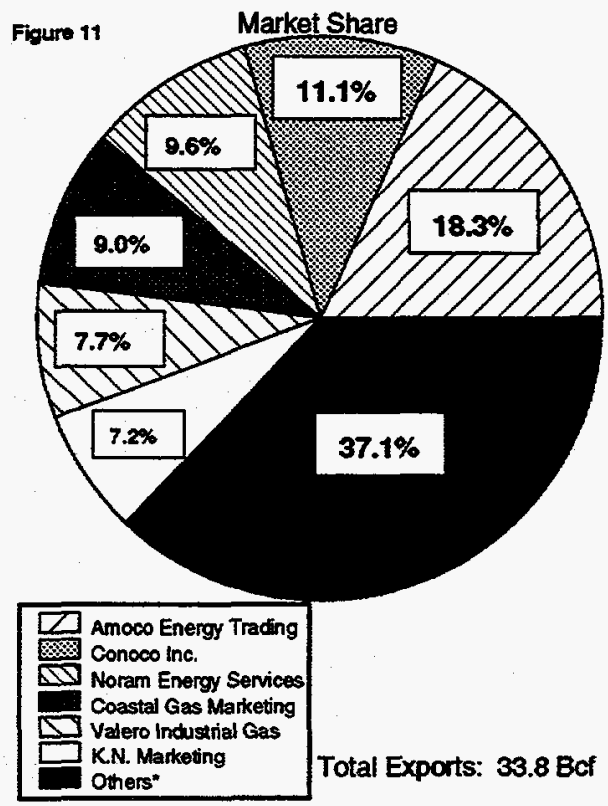

Others*

AIG Trading Corporation Anadarko Trading Company

Burlington Resources Trading inc. Channel Gas Marketing Company

Chevron U.S.A. Inc.

Coral Energy Resources

Eastex Hydrocarbons

El Paso Gas Marketing Company

Enron Capital \& Trade Resources Corp.

Gulf Energy Marketing Company

Louis Dreyfus Energy Corporation

Midcon Texas Gas Services Corporation

Mobil Natural Gas Inc.

Natural Gas Energy \& Trade, L.L.C.

Natural Gas Clearinghouse

Onyx Gas Marketing Company, L.C.

PanEnergy Gas Services, Inc.

Phibro Inc.

Texaco Natural Gas Inc.

Tristar Natural Gas inc.

Union Pacific Fuels, inc.

West Texas Gas, Inc.

Williams Energy Services Company

Figure 12

FIRMS THAT IMPORTED NATURAL GAS FROM MEXICO IN 1996

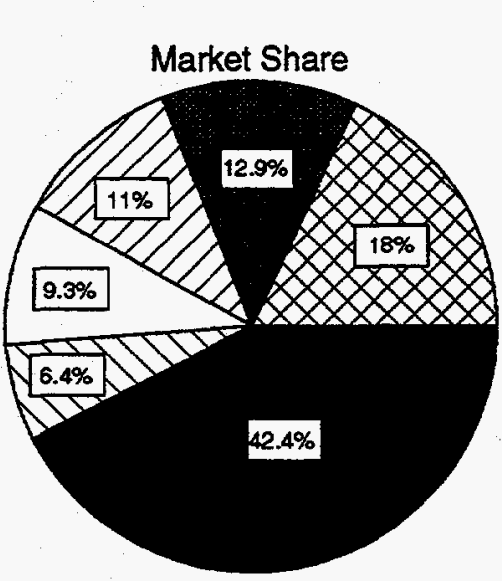

Total Imports: 13.9 Bcf
Amoco Energy Trading Corporation - Anadarko Trading Company ED Coastal Gas Marketing Company $\square$ El Paso Energy Marketing Company Corpus Christi Gas Marketing, L.P. Others"

\section{Others"}

AG Trading Corporation

Channel Gas Marketing Company

CNG Energy Services Corporation

Coral Energy Resources, L.P.

Enron Capital \& Trade Resources Corporation

Gulf Energy Marketing Company

Mobil Natural Gas Inc.

Natural Gas Clearinghouse

Noram Energy Services, Inc.

Onyx Gas Marketing Company, L.C.

PanEnergy Gas Services, inc.

Phibro Inc.

Texaco Natural Gas inc.

Union Pacific Fuels, Inc.

Valero Gas Marketing, L.P. 
Figure 13

\section{NATURAL GAS EXPORTS TO MEXICO}

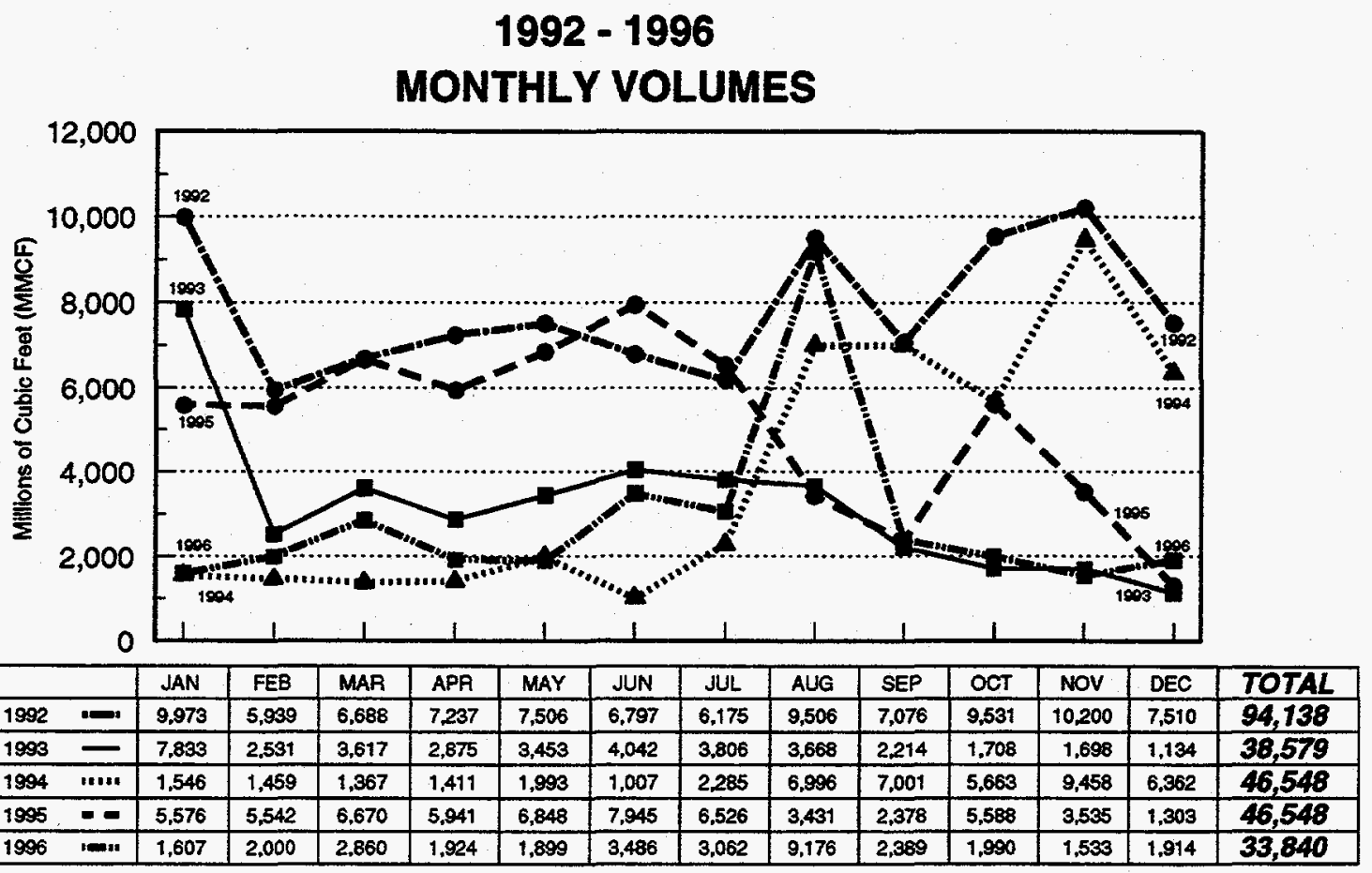

Figure 14

NATURAL GAS EXPORTS TO MEXICO

$1992-1996$

WEIGHTED AVERAGE PRICE

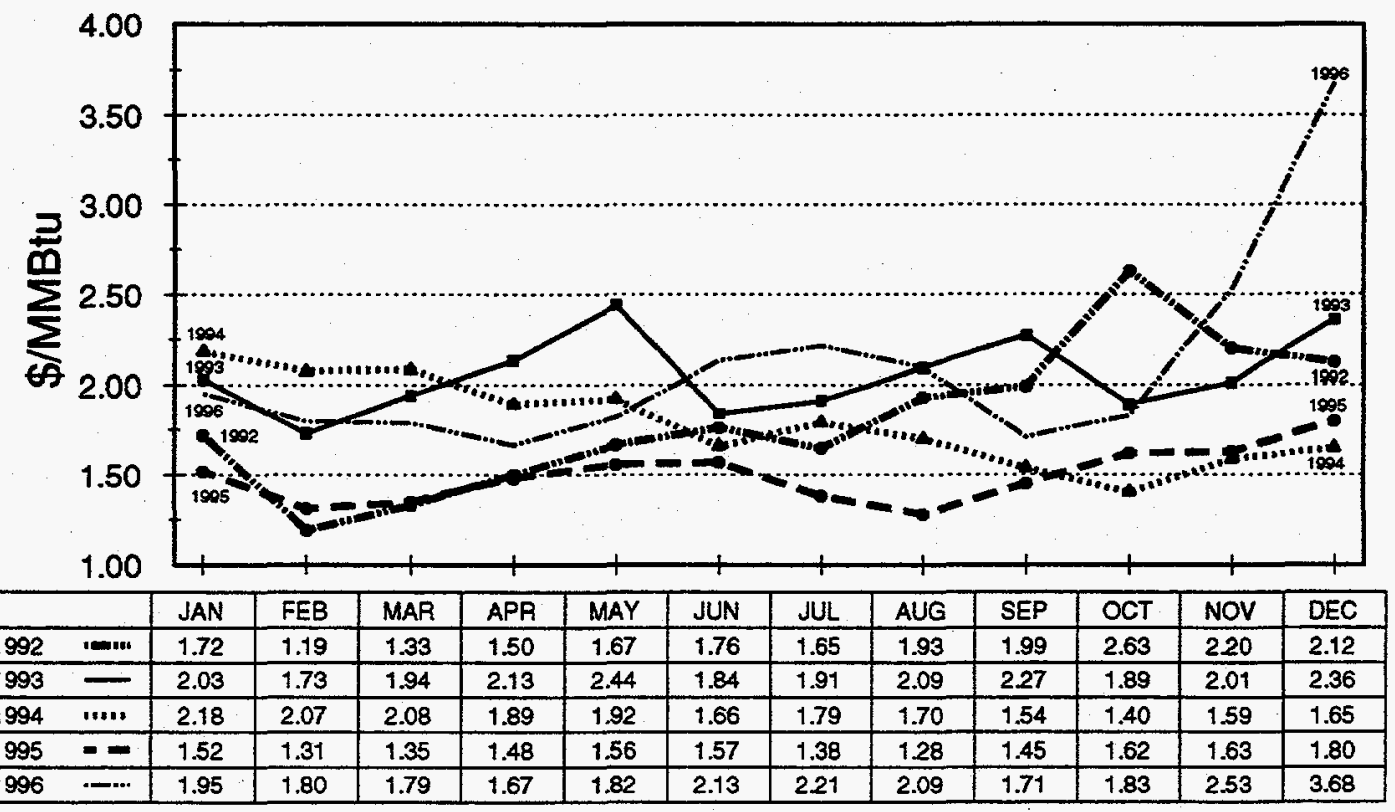

North American Gas Trade * North American Gas Trade * North American Gas Trade * North American Gas Trade * North American Gas Trade 
Page - 1

\section{LONG-TERM IMPORTS}

ACTUAL IMPORTS AS A PERCENT OF AUTHORIZED VOLUMES

\author{
IMPORTER/EXPORTER
}

AG-ENERGY, L.P./Anderson Exploration Litd.

BAY STATE GAS COMPANY/Renaissance Energy Ltd.

BOSTON GAS COMPANY/Alberta NE Gas (TCGS Ltd. 1)

BOSTON GAS COMPANY/Esso Resources Canada Limited

BOUNDARY GAS, INC./TransCanada Pipelines Limited

BROOKL.YN NAVY YARD COGEN. PARTNERS, L.P./Crestar Energy

BROOKLYYN NAVY YARD COGEN. PARTNERS, L.P.JPanCanadian Petroleum Ltd.

BROOKLYN UNION GAS COMPANY/Alberta NE Gas (AEC Oil \& Gas Company)

BROOKLYN UNION GAS COMPANY/Alberta NE Gas (TCGS LId. 1)

BROOKLYN UNION GAS COMPANY/Alberta NE Gas (TCGS LId. 2)

BROOKLYN UNION GAS COMPANY/Alberta Northeast Gas (ProGas)

BROOKLYN UNION GAS COMPANY/Alberta Northeast Gas (Producers Marketing)

CASCADE NATURAL GAS COPORATION/IGI Resources, Inc.

CASCADE NATURAL GAS CORPORATIONMMobil Oil Canada, Lid.

CASCADE NATURAL GAS CORPORATION/Westcoast Gas Services

CASCADE NATURAL GAS CORPORATION/Westcoast Gas Services

CASCADE NATURAL GAS CORPORATION/Westcoast Gas Services

CENTRAL HUDSON COMPANY/Alberta NE Gas (AEC Oil \& Gas Company)

CENTRAL HUDSON COMPANY/Alberta NE Gas (TCGS Ltd. 1)

CENTRAL HUDSON COMPANY/Alberta NE Gas (TCGS Ltd. 2)

CENTRAL HUDSON COMPANY/Alberta Northeast Gas (ProGas)

CENTRAL HUDSON COMPANY/Alberta Northeast Gas (Producers Marketing)

CHEVRON NATURAL GAS SERVICES, INC./Chevron Canada Resources

CHEVRON U.S.A. INC./Chevron Canada Resources

CITY OF BURBANK, CALIFORNIA/Westcoast Gas Services

CITY OF GLENDALE, CALIFORNIAWestcoast Gas Services

CITY OF PASADENA, CALIFORNIAWUestcoast Gas Services

COASTAL GAS MARKETING COMPANYMMorgan Hydrocarbons Inc.

COASTAL GAS MARKETING COMPANYMMorison Petroleums Ltd.

COASTAL GAS MARKETING COMPANY/Petro-Canada Resources

COGEN ENERGY TECHNOLOGY L.P./Shell Canada Limited

COLONIAL NATURAL GAS/Alberta NE Gas (TCGS Litd. 1)

COMMONWEALTH GAS COMPANY/Alberta NE Gas (TCGS Lid. 1)

CONNECTICUT NATURAL GAS/Alberta NE Gas (AEC Oil \& Gas Company)

CONNECTICUT NATURAL GAS/Alberta NE Gas (TCGS Ltd. 1)

\begin{tabular}{|c|c|c|c|c|}
\hline $\begin{array}{c}1996 \\
\text { qtr } \\
\# 1\end{array}$ & $\begin{array}{c}1996 \\
\text { qtr } \\
\# 2\end{array}$ & $\begin{array}{c}1996 \\
\text { qtr } \\
\# 3\end{array}$ & $\begin{array}{c}1996 \\
\text { qtr } \\
\# 4\end{array}$ & 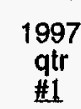 \\
\hline 86 & 84 & 83 & 86 & 9 \\
\hline 101 & 100 & 99 & 100 & 10 \\
\hline 100 & 100 & 99 & 100 & \\
\hline 100 & 100 & 99 & 100 & 9 \\
\hline 100 & 98 & 99 & 100 & 9 \\
\hline$N / A$ & N/A & $N / A$ & 109 & 10 \\
\hline$N / A$ & $N / A$ & $N / A$ & 109 & 10 \\
\hline 99 & 99 & 99 & 99 & 9 \\
\hline 100 & 100 & 100 & 100 & 100 \\
\hline 100 & 96 & 100 & 100 & 100 \\
\hline 100 & 100 & 100 & 100 & 100 \\
\hline 100 & 100 & 100 & 100 & 10 \\
\hline 99 & N/A & $\mathrm{N} / \mathrm{A}$ & 98 & 5 \\
\hline 96 & 92 & 96 & 96 & \\
\hline 54 & 63 & 77 & 77 & 7 \\
\hline 95 & 98 & 95 & 98 & \\
\hline 100 & 100 & 100 & 100 & 100 \\
\hline 96 & 96 & 96 & 96 & 9 \\
\hline 100 & 100 & 100 & 100 & 10 \\
\hline 100 & 96 & 100 & 100 & 10 \\
\hline 102 & 102 & 102 & 102 & 10 \\
\hline 99 & 99 & 99 & 99 & \\
\hline$N / A$ & N/A & 73 & 75 & \\
\hline $\mathrm{N} / \mathrm{A}$ & $N / A$ & 98 & 101 & \\
\hline 83 & 98 & 101 & 99 & \\
\hline 95 & 96 & 97 & 97 & \\
\hline 94 & 98 & 88 & 99 & \\
\hline N/A & 102 & 98 & 99 & \\
\hline N/A & $\mathrm{N} / \mathrm{A}$ & $N / A$ & 99 & \\
\hline $\mathrm{N} / \mathrm{A}$ & $\mathrm{N} / \mathrm{A}$ & N/A & 98 & \\
\hline 103 & 80 & 85 & 90 & 10 \\
\hline 100 & 100 & 100 & 100 & 10 \\
\hline 100 & 100 & 99 & 100 & 10 \\
\hline 101 & 101 & 101 & 101 & 10 \\
\hline 100 & 100 & 100 & 100 & 10 \\
\hline
\end{tabular}

WEIGHTED AVERAGE PRICE (\$MMBTU) OF IMPORTS

\begin{tabular}{|c|c|c|c|c|}
\hline $\begin{array}{c}1996 \\
\text { qtr } \\
\# 1\end{array}$ & $\begin{array}{c}1996 \\
\text { qtr } \\
\# 2\end{array}$ & $\begin{array}{c}1996 \\
\text { qtr } \\
\# 3\end{array}$ & $\begin{array}{c}1996 \\
\text { qtr } \\
\# 4\end{array}$ & $\begin{array}{c}1997 \\
\text { qtr } \\
\# 1\end{array}$ \\
\hline 2.32 & 2.31 & 2.31 & 2.36 & 2.39 \\
\hline 3.19 & 3.36 & 2.87 & 3.47 & 3.57 \\
\hline 3.07 & 2.67 & 2.43 & 3.17 & 3.29 \\
\hline 3.35 & 3.03 & 2.89 & 3.65 & 3.77 \\
\hline 3.06 & 2.75 & 2.49 & 3.26 & 3.38 \\
\hline N/A & N/A & N/A & 3.27 & 3.60 \\
\hline N/A & N/A & N/A & 3.21 & 3.54 \\
\hline 3.07 & 2.67 & 2.42 & 3.17 & 3.29 \\
\hline 3.07 & 2.67 & 2.43 & 3.17 & 3.29 \\
\hline 3.07 & 2.67 & 2.43 & 3.17 & 3.29 \\
\hline 2.96 & 2.76 & 2.50 & 3.11 & 3.26 \\
\hline 3.08 & 2.66 & 2.42 & 3.17 & 3.29 \\
\hline 1.24 & N/A & N/A & 1.22 & 5.01 \\
\hline 1.30 & 1.00 & 1.10 & 2.60 & 2.77 \\
\hline 1.66 & 1.43 & 1.29 & 2.18 & 2.60 \\
\hline 1.61 & 1.34 & 1.41 & 2.57 & 2.83 \\
\hline 1.29 & 0.99 & 1.05 & 2.54 & 2.65 \\
\hline 3.07 & 2.67 & 2.42 & 2.84 & 3.29 \\
\hline 3.07 & 2.67 & 2.43 & 3.17 & 3.29 \\
\hline 3.07 & 2.67 & 2.43 & 3.17 & 3.29 \\
\hline 2.96 & 2.76 & 2.50 & 3.11 & 3.26 \\
\hline 3.08 & 2.66 & 2.42 & 3.17 & 3.29 \\
\hline N/A & N/A & 2.15 & 2.23 & 2.66 \\
\hline N/A & N/A & 0.97 & 1.98 & 2.13 \\
\hline 1.50 & 1.31 & 1.38 & 1.44 & 1.54 \\
\hline 1.49 & 1.28 & 1.21 & 1.45 & 1.55 \\
\hline 1.39 & 1.21 & 1.15 & 1.38 & 1.47 \\
\hline N/A & 2.68 & 2.51 & 1.35 & 3.79 \\
\hline N/A & N/A & N/A & 2.17 & 3.05 \\
\hline N/A & N/A & N/A & 2.18 & 3.03 \\
\hline 2.97 & 2.97 & 2.97 & 2.97 & 2.97 \\
\hline 3.07 & 2.67 & 2.43 & 3.17 & 3.29 \\
\hline 3.07 & 2.67 & 2.43 & 3.17 & 3.29 \\
\hline 3.07 & 2.67 & 2.42 & 3.17 & 3.29 \\
\hline 3.07 & 2.67 & 2.43 & 3.17 & 3.29 \\
\hline
\end{tabular}




\section{LONG-TERM IMPORTS}

ACTUAL IMPORTS AS A PERCENT OF AUTHORIZED VOLUMES

IMPORTERIEXPORTER

CONNECTICUT NATURAL GAS/Alberta NE Gas (TCGS Ltd. 2) CONNECTICUT NATURAL GAS/Alberta Northeast Gas (ProGas) CONNECTICUT NATURAL GAS/Alberta Northeast Gas (Producers Marketing) CONSOLIDATED EDISON COMPANY OF N.Y./Alberta NE Gas (AEC Oil \& Gas) CONSOLIDATED EDISON COMPANY OF N.Y./Alberta NE Gas (ProGas) CONSOLIDATED EDISON COMPANY OF N.Y./Alberta NE Gas (Producers Marketing) CONSOLIDATED EDISON COMPANY OF N.Y./Alberta NE Gas (TCGS Ltd. 1) CONSOLIDATED EDISON COMPANY OF N.Y./Alberta NE Gas (TCGS Ltd. 2) CONSOLIDATED EDISON COMPANY OF N.Y.IAMOCo Canada CONSUMERS POWER COMPANY/Norcen Energy Resources CONSUMERS POWER COMPANY/North Canadian Oils CRESTAR ENERGY MARKETING CORPORATION/Crestar Energy CRESTAR ENERGY MARKETING CORPORATION/Crestar Energy DARTMOUTH POWER ASSOCIATES, L.P./Anderson Exploration Ltd. DARTMOUTH POWER ASSOCIATES, L.P./BXL Energy Ltd. DARTMOUTH POWER ASSOCIATES, L.P./Crestar Energy Inc. DARTMOUTH POWER ASSOCIATES, L.P./Remington Energy Ltd. DISTRIGAS CORPORATION (88-37-LNG)/Sonatrach DISTRIGAS CORPORATION (89-16-LNG)/Sonatrach EASTERN ENERGY MARKETING, INC./Talisman Energy inc. EASTERN ENERGY MARKETING, INC.Tralisman Energy Inc. ELIZABETHTOWN GAS COMPANYITCGS LId.

ENCOGEN FOUR PARTNERS, L.P./Sceptre Resources Limited ENCOGEN NORTHWEST, L.P./CanWest Gas Supply, Inc. ENERGYNORTH, INC./Alberta NE Gas (TCGS Ltd. 1) ESSEX COUNTY GAS CO./Alberta NE Gas (TCGS Lid. 1) FULTON COGENERATION ASSOCIATES/Star OII \& Gas Limited GRANITE STATE GAS TRANSMISSION, INC./Direct Energy Marketing LId. GRANITE STATE GAS TRANSMISSION, INC./Shell Canada Limited GRANITE STATE GAS TRANSMISSION, INC.IShell Canada Limited GREAT FALLS GAS COMPANY/Shell Canada Limited HERMISTON GENERATING COMPANY, L.P./CanStates Gas Marketing HERMISTON GENERATING COMPANY, L.P.IHome Oil Company Limited IGI RESOURCES, INC.Mobil Oil Canada

INDECK ENERGY SERVICES OF OSWEGO, INC./Indeck Gas Supply Corp.

\begin{tabular}{|c|c|c|c|c|}
\hline $\begin{array}{c}1996 \\
\text { qtr } \\
\# 1\end{array}$ & $\begin{array}{c}1996 \\
\text { qur } \\
\# 2\end{array}$ & $\begin{array}{c}1996 \\
\text { qtr } \\
\# 3\end{array}$ & $\begin{array}{c}1996 \\
\text { qtr } \\
\# 4\end{array}$ & $\begin{array}{c}1997 \\
\text { qtr } \\
\# 1\end{array}$ \\
\hline 99 & 95 & 99 & 99 & 99 \\
\hline 100 & 100 & 100 & 100 & 100 \\
\hline 101 & 101 & 101 & 101 & 101 \\
\hline 101 & 101 & 101 & 101 & 101 \\
\hline 100 & 100 & 100 & 100 & 100 \\
\hline 100 & 100 & 100 & 100 & 100 \\
\hline 100 & 100 & 100 & 100 & 99 \\
\hline 100 & 96 & 100 & 100 & 100 \\
\hline 100 & 100 & 100 & 100 & 100 \\
\hline 100 & 100 & 100 & 124 & 171 \\
\hline 71 & 71 & 71 & 72 & N/A \\
\hline 99 & 84 & 54 & 95 & 98 \\
\hline N/A & 20 & 99 & 100 & 101 \\
\hline 78 & 106 & 113 & 113 & 118 \\
\hline N/A & N/A & N/A & N/A & N/A \\
\hline 72 & 73 & 83 & 83 & 89 \\
\hline 78 & 76 & 82 & 81 & 89 \\
\hline$N / A$ & N/A & N/A & N/A & $N / A$ \\
\hline N/A & $\mathrm{N} / \mathrm{A}$ & N/A & $\mathrm{N} / \mathrm{A}$ & N/A \\
\hline N/A & $\mathrm{N} / \mathrm{A}$ & N/A & 60 & 95 \\
\hline N/A & N/A & N/A & 74 & 94 \\
\hline 100 & 100 & 100 & 100 & 100 \\
\hline 96 & 95 & 96 & 96 & 98 \\
\hline 43 & 37 & 61 & 81 & 88 \\
\hline 100 & 100 & 100 & 100 & 100 \\
\hline 100 & 100 & 100 & 100 & 100 \\
\hline 99 & 99 & 100 & 100 & 94 \\
\hline 101 & 101 & 48 & 101 & 102 \\
\hline 100 & 100 & 99 & 100 & 101 \\
\hline 99 & 100 & 99 & 99 & 100 \\
\hline 100 & 100 & 100 & 99 & 99 \\
\hline N/A & N/A & 97 & 83 & 39 \\
\hline N/A & N/A & 97 & 83 & 39 \\
\hline N/A & N/A & N/A & N/A & 0 \\
\hline 78 & 72 & 79 & 79 & 79 \\
\hline
\end{tabular}

\section{WEIGHTED AVERAGE PRICE (\$MMBTU) OF IMPORTS}

\begin{tabular}{|c|c|c|c|c|}
\hline $\begin{array}{c}1996 \\
\text { qtr } \\
\# 1\end{array}$ & $\begin{array}{l}1996 \\
\text { qtr } \\
\# 2\end{array}$ & $\begin{array}{c}1996 \\
\text { qtr } \\
\# 3\end{array}$ & $\begin{array}{c}1996 \\
\text { qtr } \\
\# 4\end{array}$ & $\begin{array}{c}1997 \\
\text { qtr } \\
\# 1\end{array}$ \\
\hline 3.07 & 2.67 & 2.43 & 3.17 & 3.29 \\
\hline 2.96 & 2.76 & 2.50 & 3.11 & 3.26 \\
\hline 3.08 & 2.66 & 2.42 & 3.17 & 3.29 \\
\hline 3.07 & 2.67 & 2.42 & 3.17 & 3.29 \\
\hline 2.96 & 2.76 & 2.50 & 3.11 & 3.26 \\
\hline 3.08 & 2.66 & 2.42 & 3.17 & 3.29 \\
\hline 3.07 & 2.67 & 2.43 & 3.17 & 3.30 \\
\hline 3.07 & 2.67 & 2.43 & 3.17 & 3.29 \\
\hline 2.91 & 2.85 & 2.77 & 2.31 & 3.98 \\
\hline 2.10 & 2.10 & 2.20 & 2.20 & 2.20 \\
\hline 2.10 & 2.10 & 2.20 & 2.20 & N/A \\
\hline 2.18 & 2.44 & 2.46 & 2.84 & 3.38 \\
\hline N/A & 2.15 & 1.74 & 2.32 & 2.44 \\
\hline 2.65 & 2.66 & 2.45 & 2.29 & 3.02 \\
\hline$N / A$ & N/A & N/A & N/A & 1.63 \\
\hline 3.01 & 2.66 & 2.45 & 2.28 & 3.02 \\
\hline 3.01 & 2.66 & 2.45 & 2.28 & 3.02 \\
\hline 2.63 & 2.33 & 2.53 & 2.70 & 2.85 \\
\hline N/A & N/A & N/A & $N / A$ & N/A \\
\hline N/A & N/A & N/A & 2.12 & 1.97 \\
\hline N/A & N/A & N/A & 2.12 & 1.92 \\
\hline 2.38 & 3.05 & 3.06 & 3.10 & 3.43 \\
\hline 2.90 & 2.44 & 2.73 & 2.75 & 2.75 \\
\hline 2.03 & 2.03 & 2.13 & 2.13 & 2.13 \\
\hline 3.07 & 2.67 & 2.43 & 3.17 & 3.29 \\
\hline 3.07 & 2.67 & 2.43 & 3.17 & 3.29 \\
\hline 2.51 & 2.56 & 2.55 & 2.83 & 3.09 \\
\hline 2.52 & 2.44 & 2.50 & 2.30 & 2.29 \\
\hline 3.09 & 2.84 & 2.80 & 3.46 & 3.46 \\
\hline 3.21 & 3.09 & 2.63 & 3.17 & 3.16 \\
\hline 1.22 & 1.04 & 0.96 & 1.14 & 1.19 \\
\hline N/A & N/A & 2.58 & 2.67 & 3.44 \\
\hline N/A & N/A & 2.58 & 2.67 & 3.44 \\
\hline N/A & $N / A$ & N/A & N/A & N/A \\
\hline 2.69 & 2.69 & 2.72 & 2.74 & 2.78 \\
\hline
\end{tabular}




\section{LONG-TERM IMPORTS}

\section{ACTUAL IMPORTS AS A PERCENT OF AUTHORIZED VOLUMES}

\section{IMPORTER/EXPORTER}

INDECK-YERKES ENERGY SERVICES, INC./Indeck Gas Supply Corp. INDECK-YERKES LTD. PARTNERSHIPITalisman Energy Inc.

KAMINE/BESICORP BEAVER FALLS L.P./North Canadian Marketing Inc. KAMINE/BESICORP CARTHAGE L.P./Renaissance Energy Ltd.

KAMINE/BESICORP NATURAL DAM L.P./North Canadian Markeling Inc. KAMINE/BESICORP SOUTH GLENS FALLS L.P./Renaissance Energy Ltd. KAMINE/BESICORP SYRACUSE L.P./North Canadian Marketing Inc. KCS ENERGY MARKETING, INC./Ramarro Resources Ltd.

LG\&E WESTMORELAND RENSSELAER/TCGS Ltd.

LOCKPORT ENERGY ASSOCIATES, L.P.IProGas Limited

LONG ISLAND LIGHTING COMPANYIAlberta NE Gas (AEC Oil \& Gas)

LONG ISLAND LIGHTING COMPANY/Alberta NE Gas (TCGS Ltd. 1)

LONG ISLAND LIGHTING COMPANY/Alberta NE Gas (TCGS Ltd. 2)

LONG ISLAND LIGHTING COMPANY/Alberta Northeast Gas (ProGas)

LONG ISLAND LIGHTING COMPANY/Alberta Northeast Gas (Producers Marketing)

MEGAN-RACINE ASSOCIATES, INC./TCGS Ltd.

MICHIGAN CONSOLIDATED GAS COMPANYITCGS Ltd.

MIDLAND COGENERATION VENTURE1/Norcen Energy Resources Ltd.

MIDLAND COGENERATION VENTURE2/Shell Canada Limited

MIDLAND COGENERATION VENTURE3/Canterra Energy Limited

MIDLAND COGENERATION VENTURE4/TransCanada Pipelines Limiled

MIDLAND COGENERATION VENTURE5/Poco Petroleums Lit.

MIDLAND COGENERATION VENTURE6/North Canadian Oils, Litd.

MINNEGASCO, INC./TransCanada Pipelines Limited

N.Y. STATE ELECTRIC \& GAS CO./Alberta NE Gas (AEC Oil \& Gas Co.)

N.Y. STATE ELECTRIC \& GAS CO./Alberta NE Gas (TCGS Ltd. 2)

N.Y. STATE ELECTRIC \& GAS CO./Alberta Northeast Gas (ProGas)

N.Y. STATE ELECTRIC \& GAS CO./Alberta Northeast Gas (Producers Marketing)

N.Y. STATE ELECTRIC \& GAS CO./Crestar Energy

N.Y. STATE ELECTRIC \& GAS CO./Progas Limited

NATIONAL FUEL GAS DISTRIBUTION CORP./Alberta NE Gas (TCGS Ltd.)

NATIONAL STEEL. CORPORATION/Direct Energy Marketing, Ltd.

NEW ENGLAND POWER COMPANY/Canadian Natural Resources

NEW ENGLAND POWER COMPANY/Renaissance Energy Limited

NEW JERSEY NATURAL GAS COMPANYIAlberta NE Gas (AEC Oil \& Gas Company)

$\begin{array}{rrrrr}1996 & 1996 & 1996 & 1996 & 1997 \\ \text { qtr } & \text { qtr } & \text { qtr } & \text { qtr } & \text { qtr } \\ \# 1 & \# 2 & \# 3 & \text { \#4 } & \text { \#1 } \\ & & & & \\ 73 & 73 & 73 & 75 & 75 \\ \text { N/A } & \text { N/A } & \text { N/A } & \text { N/A } & \text { N/A } \\ 5 & 6 & 5 & 6 & 6 \\ 42 & 58 & 55 & 50 & 42 \\ 3 & 4 & 4 & 4 & 4 \\ 67 & 55 & 55 & 47 & 38 \\ 3 & 3 & \text { N/A } & 3 & 3 \\ \text { N/A } & \text { N/A } & 0 & \text { N/A } & \text { N/A } \\ 100 & 100 & 100 & 100 & 100 \\ 98 & 85 & 74 & 90 & 98 \\ 99 & 99 & 99 & 99 & 99 \\ 100 & 100 & 100 & 100 & 100 \\ 100 & 96 & 100 & 100 & 100 \\ 100 & 100 & 100 & 100 & 100 \\ 99 & 99 & 99 & 99 & 99 \\ 96 & 45 & \text { N/A } & 3 & 3 \\ 93 & 94 & 92 & 94 & \text { N/A } \\ 100 & 100 & 100 & 100 & 100 \\ 100 & 100 & 100 & 100 & 100 \\ 100 & 100 & 100 & 100 & 100 \\ 100 & 100 & 100 & 100 & 100 \\ 100 & 100 & 73 & 83 & 84 \\ 100 & 75 & 19 & 39 & 100 \\ 97 & 74 & 68 & 94 & 99 \\ 102 & 102 & 102 & 102 & 102 \\ 99 & 95 & 99 & 99 & 99 \\ 101 & 101 & 101 & 101 & 101 \\ 99 & 99 & 99 & 99 & 99 \\ 101 & 96 & 100 & 100 & 91 \\ 89 & 90 & 90 & 77 & 63 \\ 100 & 100 & 100 & 100 & 92 \\ 155 & 103 & 88 & 101 & 100 \\ 43 & 43 & 41 & 41 & 41 \\ 100 & 101 & 108 & 110 & 110 \\ 102 & 102 & 102 & 102 & 102\end{array}$

WEIGHTED AVERAGE PRICE (\$MMBTU) OF IMPOATS

\begin{tabular}{|c|c|c|c|c|}
\hline $\begin{array}{c}1996 \\
\text { qtr } \\
\# 1\end{array}$ & $\begin{array}{c}1996 \\
\text { qtr } \\
\# 2\end{array}$ & 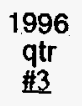 & $\begin{array}{c}1996 \\
\text { qtr } \\
\# 4\end{array}$ & $\begin{array}{c}1997 \\
\text { qtr } \\
\# 1\end{array}$ \\
\hline 2.37 & 2.38 & 2.32 & 2.36 & 2.55 \\
\hline N/A & 2.25 & 2.25 & 2.32 & 2.33 \\
\hline 3.23 & 3.21 & 3.53 & 3.31 & 3.64 \\
\hline 3.57 & 3.46 & 3.32 & 3.46 & 3.77 \\
\hline 3.03 & 3.01 & 2.99 & 3.03 & 3.58 \\
\hline 2.54 & 2.57 & 2.39 & 2.27 & 2.49 \\
\hline 3.02 & 3.03 & N/A & 3.07 & 3,54 \\
\hline N/A & N/A & N/A & N/A & N/A \\
\hline 3.02 & 2.80 & 2.49 & 3.04 & 3.06 \\
\hline 3.51 & 3.71 & 3.87 & 3.75 & 3.73 \\
\hline 3.07 & 2.67 & 2.42 & 3.17 & 3.29 \\
\hline 3.07 & 2.67 & 2.43 & 3.17 & 3.29 \\
\hline 3.07 & 2.67 & 2.43 & 3.17 & 3.29 \\
\hline 2.96 & 2.76 & 2.50 & 3.11 & 3.26 \\
\hline 3.08 & 2.66 & 2.42 & 3.17 & 3.29 \\
\hline 2.65 & 3.84 & N/A & 3.14 & 4.13 \\
\hline 2.50 & 2.26 & 2.12 & 1.72 & N/A \\
\hline 1.78 & 1.80 & 1.79 & 1.80 & 1.80 \\
\hline 1.69 & 1.69 & 1.71 & 1.71 & 1.72 \\
\hline 1.90 & 1.90 & 1.89 & 1.90 & 1.90 \\
\hline 1.69 & 1.70 & 1.70 & 1.70 & 1.71 \\
\hline 2.08 & 2.14 & 2.34 & 2.29 & 2.32 \\
\hline 2.58 & 2.48 & 4.30 & 3.58 & 2.83 \\
\hline 2.38 & 2.38 & 2.26 & 3.12 & 3.34 \\
\hline 3.07 & 2.67 & 2.42 & 3.17 & 3.29 \\
\hline 3.07 & 2.67 & 2.43 & 3.17 & 3.29 \\
\hline 2.96 & 2.76 & 2.50 & 3.11 & 3.26 \\
\hline 3.08 & 2.66 & 2.42 & 3.17 & 3.29 \\
\hline 2.57 & 2.43 & 2.39 & 2.64 & 3.02 \\
\hline 3.00 & 3.01 & 2.96 & 3.14 & 3.41 \\
\hline 3.06 & 2.65 & 2.42 & 3.17 & 3.28 \\
\hline 2.69 & 2.47 & 2.61 & 2.63 & 3.35 \\
\hline 2.54 & 2.38 & 2.26 & 2.23 & 2.41 \\
\hline 2.93 & 2.88 & 2.68 & 2.62 & 2.76 \\
\hline 3.07 & 2.67 & 2.42 & 3.17 & 3.29 \\
\hline
\end{tabular}




\section{LONG-TERM IMPORTS}

ACTUALIMPORTS AS A PERCENT OF AUTHORIZED VOLUMES

IMPORTER/EXPORTER

NEW JERSEY NATURAL GAS COMPANY/Alberta NE Gas (TCGS Ltd. 1 ) NEW JERSEY NATURAL GAS COMPANYIAlberta NE Gas (TCGS Ltd. 2) NEW JERSEY NATURAL GAS COMPANY/Alberta Northeast Gas (ProGas) NEW JERSEY NATURAL GAS COMPANY/Alberta Northeast Gas (Producers Mktg.) NIAGARA MOHAWK POWER CORPORATION/TCGS Ltd.

NORTH CANADIAN MARKETING CORPORATION/North Canadian Marketing Inc. NORTH JERSEY ENERGY ASSOCIATES/ProGas Limiled

NORTHEAST ENERGY ASSOCIATES/ProGas Limited

NORTHERN MINNESOTA UTILITIES/TCGS LId.

NORTHERN MINNESOTA UTILITIES - EASTERN MARKET/TCGS Ltd. NORTHERN MINNESOTA UTILITIES - WESTERN MARKET/TCGS LId. NORTHERN STATES POWER COMPANYIAMOco Canada (WI) NORTHERN STATES POWER COMPANYICanadian Occidental (WI) NORTHERN STATES POWER COMPANY/ProGas Limited (WI) NORTHERN STATES POWER COMPANY (MINNESOTA)/Amoco Canada (MN) NORTHERN STATES POWER COMPANY (MINNESOTA)/TCGS LId. NORTHERN UTILITIES, INC./Renaissance Energy Ltd.

NORTHWEST NATURAL GAS COMPANY/Amoco Canada NORTHWEST NATURAL GAS COMPANY/CanWest Gas Supply NORTHWEST NATURAL GAS COMPANY/Poco Potroleums Limited NORTHWEST NATURAL GAS COMPANY/Summit Resources Limited NORTHWEST NATURAL GAS COMPANYMWestcoast Gas Services NUI CORPORATION/TCGS Ltd.

NW ALASKAN PIPELINE CO-Pacific Interstate/Pan-Alberta Gas Ltd. NW ALASKAN PIPELINE CO-Pan-Alberta Gas (I)/Pan-Alberta Gas Lid. NW ALASKAN PIPELINE CO-Pan-Alberta Gas (II)/Pan-Alberta Gas Ltd. OCEAN STATE POWER/ProGas Limited OCEAN STATE POWER I/ProGas Limited OCEAN STATE POWER IITCGS Ltd.

ORANGE AND ROCKLAND UTILITIES, INC.Mascana Energy Marketing ORCHARD GAS CORPORATION/ProGas Limited

PAN ENERGY LNG SALES, INC./Sonatrading

PAWTUCKET POWER ASSOCIATES/Anderson Exploration Ltd.

PAWTUCKET POWER ASSOCIATES/Tarragon Oil \& Gas Limited

PEOPLES NATURAL GAS COMPANYITCGS Ltd.

\begin{tabular}{|c|c|c|c|c|}
\hline $\begin{array}{c}1996 \\
\text { qtr } \\
\# 1\end{array}$ & $\begin{array}{c}1996 \\
\text { qtr } \\
\# 2\end{array}$ & $\begin{array}{c}1996 \\
\text { qtr } \\
\# 3\end{array}$ & $\begin{array}{c}1996 \\
\mathrm{qtr} \\
\# 4\end{array}$ & $\begin{array}{c}1997 \\
\text { qtr } \\
\# 1\end{array}$ \\
\hline 100 & 100 & 100 & 100 & 100 \\
\hline 99 & 95 & 99 & 99 & 99 \\
\hline 100 & 100 & 100 & 100 & 100 \\
\hline 99 & 99 & 99 & 99 & 99 \\
\hline 100 & 100 & 100 & 100 & 100 \\
\hline N/A & $N / A$ & 65 & 67 & 32 \\
\hline 92 & 92 & 92 & 93 & 93 \\
\hline 102 & 102 & 102 & 103 & 103 \\
\hline 93 & 100 & 100 & 100 & 100 \\
\hline 59 & 56 & 54 & 65 & 72 \\
\hline 159 & 46 & 27 & 105 & 131 \\
\hline 98 & 99 & 97 & 99 & 99 \\
\hline 100 & 55 & 65 & 88 & 100 \\
\hline 100 & 79 & 68 & 89 & 100 \\
\hline 98 & 84 & 58 & 93 & 98 \\
\hline 99 & 99 & 91 & 99 & 99 \\
\hline 99 & 99 & 98 & 99 & 101 \\
\hline 100 & 89 & 93 & 99 & 90 \\
\hline 99 & 88 & 82 & 97 & 80 \\
\hline 97 & 68 & 67 & 96 & 61 \\
\hline 86 & 61 & 60 & 94 & 95 \\
\hline 74 & 58 & 51 & 89 & 90 \\
\hline N/A & 97 & 54 & 92 & 77 \\
\hline 100 & 100 & 99 & 100 & 91 \\
\hline 101 & 100 & 100 & 100 & 102 \\
\hline 101 & 100 & 100 & 100 & 101 \\
\hline 100 & 100 & 100 & 100 & 100 \\
\hline 100 & 100 & 100 & 100 & 100 \\
\hline 100 & 100 & 100 & 100 & 100 \\
\hline 99 & 100 & 100 & 100 & 99 \\
\hline 100 & 100 & 95 & 100 & 89 \\
\hline N/A & N/A & N/A & 59 & 60 \\
\hline 44 & 59 & 77 & 89 & 82 \\
\hline 43 & 56 & 74 & 85 & 79 \\
\hline N/A & N/A & N/A & N/A & 0 \\
\hline
\end{tabular}

WEIGHTED AVEBAGE PBICE (\$MMBTU) OF IMPORTS

\begin{tabular}{|c|c|c|c|c|}
\hline $\begin{array}{c}1996 \\
\text { qtr } \\
\# 1\end{array}$ & $\begin{array}{c}1996 \\
\text { qtr } \\
\# 2\end{array}$ & $\begin{array}{c}1996 \\
\text { qtr } \\
\# 3\end{array}$ & $\begin{array}{c}1996 \\
\text { qtr } \\
\# 4\end{array}$ & $\begin{array}{c}1997 \\
\text { qtr } \\
\# 1\end{array}$ \\
\hline 3.07 & 2.67 & 2.43 & 3.17 & 3 \\
\hline 3.07 & 2.67 & 2.43 & 3.17 & 3. \\
\hline 2.96 & 2.76 & 2.50 & 3.11 & \\
\hline 3.08 & 2.66 & 2.42 & 3.17 & 3.2 \\
\hline 3.15 & 2.88 & 2.72 & 3.36 & 0 \\
\hline N/A & $N / A$ & 2.71 & 2.57 & \\
\hline 2.37 & 2.38 & 2.39 & 2.37 & 2.3 \\
\hline 3.12 & 3.17 & 3.19 & 3.18 & 3.2 \\
\hline 2.45 & 2.19 & 2.01 & 2.43 & 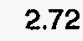 \\
\hline 1.77 & 1.52 & 1.45 & 1.90 & 2. \\
\hline 2.05 & 1.92 & 1.58 & 2.19 & 2 \\
\hline 1.62 & 1.69 & 1.69 & 1.65 & 2.3 \\
\hline 3.02 & 2.92 & 2.69 & 3.13 & 3. \\
\hline 2.42 & 2.54 & 2.52 & 3.14 & 3. \\
\hline 2.07 & 2.30 & 2.31 & 2.21 & 2.6 \\
\hline 2.12 & 2.24 & 2.20 & 2.90 & 3.2 \\
\hline 3.26 & 3.36 & 2.87 & 3.46 & 3.5 \\
\hline 1.29 & 1.22 & 1.26 & 1.86 & 1.8 \\
\hline 1.29 & 1.20 & 1.32 & 2.36 & 2.4 \\
\hline 1.15 & 1.20 & 1.25 & 1.80 & 2.8 \\
\hline 1.26 & 1.32 & 1.29 & 1.42 & 1.1 \\
\hline 1.28 & 1.33 & 1.28 & 1.38 & 1.4 \\
\hline N/A & 3.04 & 3.53 & 3.41 & $?$ \\
\hline 0.75 & 0.74 & 1.13 & 1.97 & 2. \\
\hline 1.92 & 2.14 & 2.00 & 2.49 & 2.57 \\
\hline 1.59 & 1.70 & 1.56 & 2.23 & 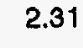 \\
\hline 2.02 & 2.07 & 2.09 & 2.16 & \\
\hline 2.02 & 2.07 & 2.09 & 2.16 & \\
\hline 2.02 & 2.07 & 2.09 & 2.16 & 2. \\
\hline 3.45 & 3.16 & 3.03 & 3.56 & \\
\hline 2.94 & 3.19 & 3.07 & 3.18 & \\
\hline N/A & $N / A$ & N/A & 2.00 & \\
\hline 4.01 & 3.44 & 3.06 & 3.02 & 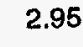 \\
\hline 4.00 & 3.39 & 3.05 & 3.23 & \\
\hline N/A & N/A & N/A & N/A & \\
\hline
\end{tabular}




\section{LONG-TERM IMPORTS}

ACTUAL IMPORTS AS A PERCENT OF AUTHORIZED VOLUMES

\begin{tabular}{|c|c|c|c|c|}
\hline $\begin{array}{c}1996 \\
\text { qtr } \\
\# 1\end{array}$ & $\begin{array}{c}1996 \\
\text { qtr } \\
\# 2\end{array}$ & $\begin{array}{c}1996 \\
\text { qtr } \\
\# 3\end{array}$ & $\begin{array}{c}1996 \\
\text { qtr } \\
\sharp 4\end{array}$ & $\begin{array}{c}1997 \\
\text { qtr } \\
\# 1\end{array}$ \\
\hline 101 & 102 & 102 & 102 & 101 \\
\hline 92 & 78 & 96 & 97 & 99 \\
\hline 75 & 79 & 96 & 97 & 99 \\
\hline 98 & 96 & 98 & 98 & 96 \\
\hline 3 & 1 & 0 & 3 & N/A \\
\hline 88 & 65 & 80 & 86 & 92 \\
\hline 100 & 100 & 100 & 100 & 100 \\
\hline 6 & 93 & 99 & 91 & 95 \\
\hline$N / A$ & N/A & N/A & 25 & 29 \\
\hline N/A & N/A & N/A & 98 & 101 \\
\hline 98 & 98 & 98 & 98 & 96 \\
\hline 97 & 89 & 98 & 126 & 96 \\
\hline N/A & N/A & N/A & N/A & N/A \\
\hline 100 & 100 & 100 & 100 & 100 \\
\hline 102 & 102 & 103 & 104 & 101 \\
\hline 100 & 100 & 100 & 100 & 100 \\
\hline 96 & 96 & N/A & 100 & 110 \\
\hline 99 & 98 & 98 & 99 & 98 \\
\hline 99 & 99 & 99 & 99 & 99 \\
\hline$N / A$ & $N / A$ & N/A & $N / A$ & 0 \\
\hline 100 & 100 & 109 & N/A & N/A \\
\hline 99 & 98 & 107 & 97 & 99 \\
\hline N/A & N/A & N/A & N/A & 0 \\
\hline 85 & 79 & 80 & 72 & 86 \\
\hline 93 & 84 & 92 & 90 & 95 \\
\hline 93 & 84 & 92 & 90 & 95 \\
\hline 49 & 47 & 44 & 59 & 48 \\
\hline 92 & 83 & 92 & 89 & 95 \\
\hline 89 & 89 & 87 & 88 & 87 \\
\hline 100 & 100 & 89 & 101 & 100 \\
\hline 68 & 67 & 68 & 68 & 68 \\
\hline 48 & 48 & 36 & 48 & 49 \\
\hline 98 & 98 & 98 & 98 & 99 \\
\hline 68 & 67 & 48 & 67 & 68 \\
\hline 99 & 99 & 99 & 99 & 99 \\
\hline
\end{tabular}

1997

1

01

99

96

N/A

92

100

29

101

96

N/A

100

101

110

98

99

0

N/A

0

86

95

48

7

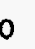

68

49

99

99
WEIGHTED AVERAGEPRICE (\$MMBTU) OE IMPORTS

\begin{tabular}{|c|c|c|c|c|}
\hline $\begin{array}{l}1996 \\
\text { qtr } \\
\# 1\end{array}$ & $\begin{array}{c}1996 \\
\text { qtr } \\
\# 2\end{array}$ & $\begin{array}{c}1996 \\
\text { qtr } \\
\# 3\end{array}$ & $\begin{array}{c}1996 \\
\text { qtr } \\
\mathbb{4}\end{array}$ & $\begin{array}{c}1997 \\
\text { qtr } \\
\# 1\end{array}$ \\
\hline 3.48 & 3.00 & 2.85 & 3.39 & 3.46 \\
\hline 2.52 & 2.56 & 2.64 & 2.89 & 2.73 \\
\hline 2.96 & 2.87 & 2.81 & 3.19 & 2.95 \\
\hline 1.28 & 1.00 & 1.04 & 2.32 & 2.58 \\
\hline 2.33 & 2.25 & 2.25 & 2.25 & N/A \\
\hline 3.36 & 3.59 & 3.45 & 3.35 & 3.61 \\
\hline 2.34 & 2.11 & 2.07 & 2.28 & 2.36 \\
\hline 2.80 & 2.59 & 2.42 & 2.81 & 3.10 \\
\hline N/A & N/A & N/A & 2.93 & 2.74 \\
\hline N/A & N/A & N/A & 2.93 & 3.14 \\
\hline 2.83 & 2.25 & 2.07 & 2.78 & 3.02 \\
\hline 2.83 & 2.31 & 2.07 & 2.72 & 3.02 \\
\hline 2.19 & 2.30 & 2.28 & 2.27 & 2.38 \\
\hline 3.07 & 2.67 & 2.43 & 3.17 & 3.29 \\
\hline 1.72 & 1.62 & 1.57 & 2.21 & 2.23 \\
\hline 3.50 & 3.03 & 2.85 & 3.42 & 3.55 \\
\hline 3.01 & 2.83 & N/A & 4.32 & 3.70 \\
\hline 1.67 & 1.74 & 1.67 & 2.01 & 2.04 \\
\hline 1.52 & 1.62 & 1.51 & 2.19 & 2.37 \\
\hline N/A & N/A & N/A & N/A & N/A \\
\hline 0.49 & 0.35 & 0.74 & $\mathrm{~N} / \mathrm{A}$ & N/A \\
\hline 0.79 & 0.72 & 1.06 & 1.96 & 2.10 \\
\hline N/A & N/A & $\mathrm{N} / \mathrm{A}$ & N/A & N/A \\
\hline 3.21 & 3.21 & 3.21 & 3.29 & 3.34 \\
\hline 2.63 & 2.58 & 2.44 & 2.77 & 2.92 \\
\hline 2.63 & 2.57 & 2.44 & 2.79 & 2.91 \\
\hline 2.62 & 2.57 & 2.43 & 2.62 & 3.29 \\
\hline 2.58 & 2.51 & 2.37 & 2.73 & 2.84 \\
\hline 3.23 & 3.22 & 3.23 & 3.23 & 3.56 \\
\hline 2.62 & 2.78 & 2.75 & 3.10 & 3.56 \\
\hline 1.17 & 1.60 & 1.54 & 1.98 & 3.13 \\
\hline 1.17 & 1.60 & 1.54 & 1.97 & 3.13 \\
\hline 1.16 & 1.57 & 1.48 & 1.65 & 3.35 \\
\hline 1.17 & 1.60 & 1.54 & 1.98 & 3.13 \\
\hline 3.07 & 2.67 & 2.42 & 3.17 & 3.29 \\
\hline
\end{tabular}

3.07
PG ENERGY $\pi$ ransCanada PipeLines Limited

PITTSFIELD GENERATING CO., L.P./Anderson Exploration Lid. PITTSFIELD GENERATING CO., L.P.Tralisman Energy Inc.

POCO PETROLEUM INC./IGI Resources, Inc.

PORTAL MUNICIPAL GAS/SaskEnergy Inc.

POWER CITY PARTNERS, L.P./Husky Oil Operations Ltd.

PROGAS U.S.A. INC./ProGas Limited

PROGAS U.S.A. INC./ProGas Limited

PROGAS U.S.A. INC./ProGas Limited

PROGAS U.S.A. INC./ProGas Limited

PROGAS U.S.A. INC. - (NATURAL GAS CLEARINGHOU/ProGas Limited

PROGAS U.S.A. INC. - (TENASKA GAS COMPANY)/ProGas Limited

PROJECT ORANGE ASSOCIATES, L.P./Noranda, Inc.

PUBLIC SERVICE ELECTRIC \& GAS/Alberta NE Gas (TCGS Ltd. 1)

RENAISSANCE ENERGY (U.S.) INC./Renaissance Energy Limited

RENAISSANCE ENERGY (U.S.) INC./Renaissance Energy Limited

ROCHESTER GAS AND ELECTRIC CORPORATION/Renaissance Energy Limited

SALMON RESOURCES LTD.-ENRON GAS MARKETING/Shell Canada Limited

SALMON RESOURCES LTD.-MIDAMERICAN ENERGY COMP/Shell Canada Limited

SAN DIEGO GAS \& ELECTRIC COMPANY/Bow Valley Industries Lid.

SAN DIEGO GAS \& ELECTRIC COMPANY/Canadian Hunter Marketing Ltd.

SAN DIEGO GAS \& ELECTRIC COMPANY/Husky Oil Operations Lid.

SAN DIEGO GAS \& ELECTRIC COMPANY/Summit Resources Lid.

SARANAC POWER PARTNERS, L.P.IShell Canada Limited

SELKIRK COGEN PARTNERS, L.P./Imperial Oil Resources Limited

SELKIRK COGEN PARTNERS, L.P./PanCanadian Petroleum Ltd.

SELKIRK COGEN PARTNERS, L.P.PParamount Resources Ltd.

SELKIRK COGEN PARTNERS, L.P.IProducers Marketing, LId.

SITHE/NDEPENDENCE POWER PARTNERS, L.P./Enron Power Services inc.

SOUTHEASTERN MICHIGAN GAS COMPANYITCGS LId.

SOUTHERN CALIFORNIA EDISON COMPANYIAEC Oil \& Gas Company

SOUTHERN CALIFORNIA EDISON COMPANY/Imperial Oil Resources Limited

SOUTHERN CALIFORNIA EDISON COMPANY/Shell Canada Limited

SOUTHERN CALIFORNIA EDISON COMPANYITCGS Ltd.

SOUTHERN CONNECTICUT GAS/Alberta NE Gas (AEC Oil \& Gas Company) 


\section{LONG-TERM IMPORTS}

\section{ACTUAL IMPORTS AS A PERCENT OF AUTHORIZED VOLUMES}

\section{IMPORTER/EXPORTER}

SOUTHERN CONNECTICUT GAS/Alberta NE Gas (TCGS Ltd. 1)

SOUTHERN CONNECTICUT GAS/Alberta NE Gas (TCGS Ltd. 2)

SOUTHERN CONNECTICUT GAS/Alberta Northeast Gas (ProGas)

SOUTHERN CONNECTICUT GAS/Alberta Northeast Gas (Producers Marketing)

ST. LAWRENCE GAS COMPANY, INC./Niagara Gas Transmission Ltd.

ST. LAWRENCE GAS COMPANY, INC./TCGS Ltd.

SUMAS COGENERATION COMPANY, L.P./ENCO Gas, Ltd.

SUMAS COGENERATION COMPANY, L.P.Mestcoast Gas Services

TENASKA GAS COMPANY/Husky Oil Operations Ltd.

TENASKA GAS COMPANY/Petro-Canada Ltd.

TENASKA GAS COMPANYITalisman Energy inc.

TENNESSEE GAS PIPELINE COMPANY/KannGaz Producers Ltd.

TENNESSEE GAS PIPELINE COMPANY/ProGas Limited

THE MONTANA POWER COMPANY/Canadian-Montana Pipeline Company

THE MONTANA POWER COMPANY/Canadian-Montana Pipeline Company

THE U.S. GENERAL SERVICES ADMIN./Chief Mountain Natural Gas Coop.

TM STAR FUEL COMPANY/CanWest Gas Supply

TRANSCO ENERGY MARKETING COMPANY/Canstates Gas Marketing

TRANSCO ENERGY MARKETING COMPANY-BG\&E/Canstates Gas Marketing

TRANSCO ENERGY MARKETING COMPANY-LILCO/Canstates Gas Marketing

TRANSCO ENERGY MARKETING COMPANY.PSE\&G/Canstates Gas Marketing

UTILICORP UNITED, INC./ProGas Limited

UTILICORP UNITED, INC./TCGS Ltd.

VALLEY GAS COMPANYIAlberta NE Gas (TCGS Ltd. 1)

VERMONT GAS SYSTEMS INC./TCGS Ltd.

VERMONT GAS SYSTEMS, INC.Narious Suppliers

WASHINGTON NATURAL GAS COMPANY/Amoco Canada

WASHINGTON NATURAL GAS COMPANY/Mobil Oil Canada

WASHINGTON NATURAL GAS COMPANY/Poco Petroleums Ltd.

WASHINGTON NATURAL GAS COMPANYMestcoast Gas Services

WASHINGTON NATURAL GAS COMPANYMUstcoast Gas Services

WASHINGTON WATER POWER COMPANYIAEC Oil \& Gas Company

WASHINGTON WATER POWER COMPANY/Amerada Hess Canada Ltd.

WASHINGTON WATER POWER COMPANY/PanCanadian Petroleum Limited

WASHINGTON WATER POWER COMPANYM Westcoast Gas Services

$\begin{array}{rrrrr}1996 & 1996 & 1996 & 1996 & 1997 \\ \text { qtr } & \text { qtr } & \text { qtr } & \text { qtr } & \text { qtr } \\ \# 1 & \# 2 & \# 3 & \# 4 & \text { \#1 } \\ & & & & \\ 150 & 150 & 150 & 150 & 150 \\ \text { N/A } & 88 & \text { N/A } & \text { N/A } & \text { N/A } \\ 100 & 100 & 100 & 100 & 100 \\ 101 & 101 & 101 & 101 & 101 \\ 55 & 20 & \text { N/A } & \text { N/A } & \text { N/A } \\ \text { N/A } & \text { N/A } & 15 & 58 & 74 \\ 82 & 79 & 77 & 92 & 88 \\ 104 & 103 & 103 & 99 & 35 \\ 20 & 15 & 73 & 76 & 96 \\ 20 & 39 & 73 & 74 & 24 \\ 22 & 10 & 91 & 91 & \text { N/A } \\ \text { N/A } & \text { N/A } & \text { N/A } & \text { N/A } & 0 \\ \text { N/A } & \text { N/A } & \text { N/A } & \text { N/A } & 0 \\ 40 & 37 & 34 & 38 & 40 \\ 20 & 17 & 19 & 17 & 16 \\ 2 & 1 & 0 & 1 & 1 \\ 93 & 70 & 66 & 94 & 95 \\ 97 & 98 & 99 & 99 & 97 \\ 62 & 51 & \text { N/A } & \text { N/A } & \text { N/A } \\ 61 & 105 & 88 & 88 & 101 \\ 59 & 93 & 76 & 76 & 99 \\ 99 & 98 & 94 & 95 & 95 \\ 100 & 99 & 97 & 100 & 100 \\ 98 & 99 & 98 & 99 & 99 \\ 100 & 65 & 42 & 82 & 100 \\ 49 & 2 & \text { N/A } & 46 & 58 \\ 104 & 103 & 102 & 104 & 92 \\ 103 & 103 & 73 & 103 & 93 \\ 89 & 102 & 104 & 104 & 95 \\ 103 & 111 & 118 & 103 & 101 \\ 61 & 79 & 75 & 80 & 81 \\ 77 & 76 & 76 & 80 & 82 \\ 60 & 62 & 62 & 65 & 55 \\ 79 & 80 & 79 & 79 & 68\end{array}$

WEIGHTED AVERAGE PRICE (\$MMBTU) OF IMPORTS

\begin{tabular}{|c|c|c|c|c|}
\hline $\begin{array}{c}1996 \\
\text { qtr } \\
\# 1\end{array}$ & $\begin{array}{c}1996 \\
\text { qtr } \\
\# 2\end{array}$ & $\begin{array}{l}1996 \\
\text { qtr } \\
\# 3\end{array}$ & $\begin{array}{l}1996 \\
\text { qtr } \\
\# 4\end{array}$ & $\begin{array}{c}1997 \\
\text { qtr } \\
\# 1\end{array}$ \\
\hline 3.07 & 2.67 & 2.43 & 3.17 & 3.29 \\
\hline$N / A$ & 2.67 & N/A & N/A & $N / A$ \\
\hline 2.96 & 2.76 & 2.50 & 3.11 & 3.26 \\
\hline 3.08 & 2.66 & 2.42 & 3.17 & 3.29 \\
\hline 2.88 & 2.15 & N/A & N/A & N/A \\
\hline N/A & N/A & 2.11 & 2.23 & 2.50 \\
\hline 2.43 & 2.43 & 2.43 & 2.18 & 2.07 \\
\hline 1.29 & 1.29 & 1.29 & 1.23 & 1.20 \\
\hline 5.32 & 4.19 & 2.20 & 2.20 & 2.31 \\
\hline 6.20 & 2.46 & 2.33 & 2.33 & 2.45 \\
\hline 1.78 & 1.83 & 1.66 & 2.00 & $N / A$ \\
\hline N/A & N/A & N/A & N/A & N/A \\
\hline N/A & N/A & N/A & N/A & $N / A$ \\
\hline 1.18 & 1.00 & 0.95 & 1.30 & 1.79 \\
\hline 1.03 & 1.02 & 1.00 & 1.03 & 1.03 \\
\hline 1.70 & 1.54 & 1.66 & 1.67 & 2.23 \\
\hline 2.06 & 2.06 & 2.06 & 2.06 & 2.14 \\
\hline 3.84 & 2.17 & 3.53 & 3.53 & 2.32 \\
\hline 3.56 & 3.63 & N/A & $N / A$ & N/A \\
\hline 3.87 & 2.87 & 3.46 & 3.46 & 3.79 \\
\hline 2.67 & 2.92 & 3.52 & 3.53 & 4.09 \\
\hline 2.53 & 2.42 & 2.33 & 2.90 & 3.08 \\
\hline 2.58 & 2.47 & 2.36 & 2.96 & 3.14 \\
\hline 3.07 & 2.67 & 2.43 & 3.18 & 3.29 \\
\hline 2.82 & 3.15 & 3.79 & 3.12 & 2.70 \\
\hline 2.00 & 20.32 & N/A & 1.84 & 1.99 \\
\hline 1.24 & 0.96 & 1.03 & 2.30 & 2.43 \\
\hline 1.23 & 0.95 & 1.30 & 2.31 & 2.45 \\
\hline 1.21 & 0.86 & 0.93 & 2.21 & 2.34 \\
\hline 1.22 & 0.91 & 1.08 & 2.31 & 2.53 \\
\hline 1.32 & 1.10 & 1.24 & 1.90 & 2.22 \\
\hline 1.20 & N/A & N/A & 1.15 & 1.15 \\
\hline 1.15 & 0.97 & 0.92 & 1.24 & 1.74 \\
\hline 1.07 & 0.98 & 0.93 & 1.26 & 1.74 \\
\hline 48 & 1.31 & 1.35 & 2.07 & 2.35 \\
\hline
\end{tabular}


Page - 7

\section{LONG-TERM IMPORTS}

\section{ACTUAL IMPORTS AS A PERCENT OE AUTHORIZED VOLUMES}

$\begin{array}{rrrrr}1996 & 1996 & 1996 & 1996 & 1997 \\ \text { qtr } & \begin{array}{c}\text { qtr } \\ \text { qtr }\end{array} & \begin{array}{c}\text { qtr } \\ \text { qtr }\end{array} & \begin{array}{r}\# 3 \\ \# 1\end{array} \\ & & & & \\ 98 & 99 & 101 & 97 & 100 \\ 105 & 106 & 108 & 109 & 106 \\ 95 & 89 & 101 & 102 & 99 \\ 101 & 99 & 101 & 102 & 86 \\ 106 & 105 & 107 & 105 & 94 \\ 102 & 100 & 102 & 103 & 100 \\ 100 & 99 & 98 & 100 & 100 \\ 99 & 100 & 96 & 100 & 100 \\ 100 & 100 & 99 & 100 & 100 \\ 99 & 99 & 74 & 100 & 85 \\ 98 & 98 & 90 & 98 & 99 \\ 100 & 100 & 97 & 101 & 95 \\ 99 & 99 & 96 & 78 & 88 \\ 100 & 100 & 100 & 100 & 100 \\ 99 & 99 & 96 & 99 & 100 \\ 98 & 98 & 63 & 97 & 88 \\ 99 & 99 & 61 & 99 & 88 \\ 100 & 100 & 98 & 100 & 100 \\ 99 & 99 & 97 & 100 & 100 \\ 99 & 99 & 96 & 99 & 100 \\ 101 & 101 & 101 & 101 & 101 \\ 100 & 100 & 100 & 100 & 100 \\ 100 & 96 & 100 & 100 & 100 \\ 100 & 100 & 100 & 100 & 100 \\ 100 & 100 & 100 & 100 & 100\end{array}$

97

100

86

94

100

00

85

99

100

100

88

100

100

100

100

100

100
WEIGHTED AVERAGE PRICE (\$MMBTU) OF IMPORTS

\begin{tabular}{|c|c|c|c|c|}
\hline $\begin{array}{l}1996 \\
\text { qtr } \\
\# 1\end{array}$ & $\begin{array}{c}1986 \\
\text { qtr } \\
\# 2\end{array}$ & $\begin{array}{c}1996 \\
\text { qtr } \\
\# 3\end{array}$ & $\begin{array}{c}1996 \\
\text { qtr } \\
\# 4\end{array}$ & 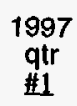 \\
\hline 1.78 & 1.82 & 1.77 & 2.11 & 2.79 \\
\hline 1.86 & 2.03 & 1.85 & 2.53 & 2.76 \\
\hline 1.87 & 2.09 & 1.69 & 2.62 & 2.89 \\
\hline 1.84 & 1.90 & 1.49 & 2.39 & 2.77 \\
\hline 1.99 & 2.08 & 1.91 & 3.55 & 2.87 \\
\hline 1.89 & 2.01 & 1.62 & 2.56 & 2.80 \\
\hline 2.47 & 2.44 & 2.38 & 2.88 & 3.06 \\
\hline 2.55 & 2.45 & 2.37 & 2.87 & 3.02 \\
\hline 2.43 & 2.33 & 2.21 & 1.99 & 2.98 \\
\hline 2.43 & 2.32 & 2.38 & 1.63 & 3.00 \\
\hline 2.53 & 2.43 & 2.34 & 2.88 & 3.07 \\
\hline 2.53 & 2.42 & 2.31 & 2.86 & 2.90 \\
\hline 2.51 & 2.42 & 2.33 & 2.86 & 3.10 \\
\hline 2.51 & 2.42 & 2.31 & 2.85 & 3.13 \\
\hline 2.53 & 2.43 & 2.30 & 2.88 & 3.07 \\
\hline 2.09 & 2.26 & 2.57 & 2.43 & 3.16 \\
\hline 2.55 & 2.45 & 2.72 & 11.93 & 3.11 \\
\hline 2.52 & 2.43 & 2.31 & 2.88 & 3.06 \\
\hline 2.53 & 2.43 & 2.31 & 2.88 & 3.06 \\
\hline 2.54 & 2.45 & 2.37 & 2.87 & 3.02 \\
\hline 3.07 & 2.67 & 2.42 & 3.17 & 3.29 \\
\hline 3.07 & 2.67 & 2.43 & 3.17 & 3.29 \\
\hline 3.07 & 2.67 & 2.43 & 3.17 & 3.29 \\
\hline 2.96 & 2.76 & 2.50 & 3.11 & 3.26 \\
\hline 3.08 & 2.66 & 2.42 & 3.17 & 3.29 \\
\hline
\end{tabular}

WESTCOAST GAS SERVICES INC. - CIBOLA CORPORATMEstcoast Gas WESTCOAST GAS SERVICES INC. - INTERSTATE POWEMEstcoast Gas WESTCOAST GAS SERVICES INC. - METRO. UTIL. DIMestcoast Gas WESTCOAST GAS SERVICES INC. - MIDAMERICAN ENE/Westcoast Gas WESTCOAST GAS SERVICES INC. - N'WESTERN PUB./Westcoast Gas WESTCOAST GAS SERVICES INC. - WISCONSIN GAS CN Westcoast Gas WISCONSIN ELECTRIC POWER CO. (GAS OPS)/Progas Limited WISCONSIN ELECTRIC POWER CO. (GAS OPS)/TCGS LId. WISCONSIN FUEL \& LIGHT COMPANY/Progas Limited WISCONSIN FUEL \& LIGHT COMPANYITCGS LId. WISCONSIN GAS COMPANYIProGas Limited (I) WISCONSIN GAS COMPANY/ProGas Limited (II) WISCONSIN GAS COMPANY/TCGS LId WISCONSIN GAS COMPANYITCGS LId WISCONSIN POWER \& LIGHT COMPANYIProGas Limited WISCONSIN POWER \& LIGHT COMPANY/TCGS LId. WISCONSIN POWER \& LIGHT COMPANYITCGS LId. WISCONSIN PUBLIC SERVICE CORPORATION/ProGas Limited WISCONSIN PUBLIC SERVICE CORPORATION/ProGas Limited WISCONSIN PUBLIC SERVICE CORPORATION/TCGS LId.

YANKEE GAS SERVICES CO./Alberta NE Gas (AEC Oil \& Gas Company) YANKEE GAS SERVICES CO./Alberta NE Gas (TCGS Ltd. 1)

YANKEE GAS SERVICES CO./Alberta NE Gas (TCGS Ltd. 2)

YANKEE GAS SERVICES CO./Alberta Northeast Gas (ProGas)

YANKEE GAS SERVICES CO./Alberta Northeast Gas (Producers Marketing) 


\section{Quarterly Attachment B}

Volumes and Weighted Average Prices

\begin{tabular}{|c|c|c|c|c|c|c|}
\hline $\begin{array}{l}\text { Year \& } \\
\text { Month }\end{array}$ & $\begin{array}{l}\text { Number of } \\
\text { Active } \\
\text { Contracts }\end{array}$ & $\begin{array}{l}\text { Est. Volumes } \\
\text { (Mcf) }\end{array}$ & $\begin{array}{c}\text { Weighted Avg. } \\
\text { Price (\$MMMtu) }\end{array}$ & $\begin{array}{l}\text { Number of } \\
\text { Active } \\
\text { Contracts }\end{array}$ & $\begin{array}{l}\text { Est. Volumes } \\
\text { (Mcf) }\end{array}$ & $\begin{array}{l}\text { Weighted Avg. } \\
\text { Price (\$MMMtu) }\end{array}$ \\
\hline \multicolumn{7}{|l|}{1995} \\
\hline January & 192 & $120,871,930$ & 1.86 & 1 & $2,510,858$ & 2.19 \\
\hline February & 196 & $110,425,827$ & 1.73 & 0 & 0 & 0.00 \\
\hline March & 193 & $119,417,643$ & 1.65 & 1 & $2,620,808$ & 2.23 \\
\hline April & 193 & $114,765,576$ & 1.65 & 0 & 0 & 0.00 \\
\hline May & 187 & $114,393,763$ & 1.72 & 0 & 0 & 0.00 \\
\hline June & 192 & $108,006,049$ & 1.75 & 0 & 0 & 0.00 \\
\hline July & 196 & $116,297,223$ & 1.65 & 0 & 0 & 0.00 \\
\hline August & 194 & $120,272,510$ & 1.60 & 1 & $2,648,297$ & 2.20 \\
\hline September & 193 & $116,543,147$ & 1.71 & 0 & 0 & 0.00 \\
\hline October & 194 & $118,728,537$ & 1.75 & 0 & 0 & 0.00 \\
\hline November & 204 & $112,259,468$ & 1.87 & 1 & $2,487,498$ & 2.25 \\
\hline December & 204 & $122,670,525$ & 2.03 & 1 & $2,501,885$ & 2.41 \\
\hline \multicolumn{7}{|l|}{1996} \\
\hline January & 202 & $123,290,469$ & 2.22 & 1 & $2,460,298$ & 2.56 \\
\hline February & 204 & $115,273,648$ & 2.11 & 1 & $2,511,896$ & 2.54 \\
\hline March & 201 & $120,563,725$ & 2.14 & 1 & $2,600,073$ & 2.79 \\
\hline April & 203 & $117,202,764$ & 2.17 & 1 & $2,599,455$ & 2.32 \\
\hline May & 202 & $118,885,146$ & 2.04 & 1 & $2,611,639$ & 2.35 \\
\hline June & 198 & $110,192,089$ & 2.06 & 0 & 0 & 0.00 \\
\hline July & 203 & $117,462,331$ & 2.15 & 1 & $2,642,277$ & 2.73 \\
\hline August & 204 & $119,938,020$ & 2.12 & 1 & $2,629,351$ & 2.33 \\
\hline September & 203 & $115,102,880$ & 1.91 & 0 & 0 & 0.00 \\
\hline October & 210 & $123,079,406$ & 1.96 & 1 & $5,114,221$ & 2.70 \\
\hline November & 215 & $123,737,301$ & 2.48 & 2 & $4,634,428$ & 2.37 \\
\hline December & 213 & $127,236,577$ & 3.20 & 2 & $4,790,631$ & 2.38 \\
\hline \multicolumn{7}{|l|}{1997} \\
\hline January & 213 & $125,351,689$ & 3.49 & 2 & $7,301,466$ & 2.51 \\
\hline February & 210 & $108,723,149$ & 2.77 & 2 & $7,165,944$ & 2.72 \\
\hline March & 205 & $121,008,720$ & 1.93 & 1 & $2,530,039$ & 2.71 \\
\hline
\end{tabular}




\section{VOLUME AND PRICE REPORT}

Long-Term Imports

Volumes (MMct/d) \& Prices ( $\$ M M M B T U$ ) of Natural

Gas Imported During the Past 12 Months 04/01/96 - 03/31/97

Long-Term Importer

AG-ENERGY, L.P

(Anderson Exploration Ltd.)

AVG Daily Quantity

Total Price (Waddington. NY)

Commodity Component

Demand Component

Reservation Fee Component

BAY STATE GAS COMPANY

(Renaissance Energy Ltd.)

AVG Daily Quantity

Total Price (Niagara Falls. NY)

Commodity Component

Demand Component

Reservation Fee Component

BOSTON GAS COMPANY

(Alberta NE Gas (TCGS Ltd. 1))

AVG Daily Quantity

Total Price (Waddington. NY)

Commodity Component

Demand Component

Reservation Fee Component

BOSTON GAS COMPANY

(Esso Resources Canada Limited)

AVG Daily Quantity

Total Price (Waddington. NY)

Commodity Component

Demand Component

Reservation Fee Component

BOUNDARY GAS, INC

(TransCanada Pipelines Limited)

AVG Daily Quantity

Total Price (Niagara Falls, NY)

Commodity Component

Demand Component

Reservation Fee Component

\begin{tabular}{|c|c|c|c|c|c|c|c|c|c|c|c|c|c|c|}
\hline $\begin{array}{l}\text { Auth. } \\
\text { Vols. }\end{array}$ & $\begin{array}{l}1996 \\
\text { Apr. }\end{array}$ & $\begin{array}{l}1996 \\
\text { May. }\end{array}$ & $\begin{array}{l}1996 \\
\text { Jun. }\end{array}$ & $\begin{array}{l}1996 \\
\text { Jul. }\end{array}$ & $\begin{array}{l}1996 \\
\text { Aug. }\end{array}$ & $\begin{array}{c}1996 \\
\text { Sepp. }\end{array}$ & $\begin{array}{l}1996 \\
\text { Oct. }\end{array}$ & $\begin{array}{l}1996 \\
\text { Nov. }\end{array}$ & $\begin{array}{l}1996 \\
\text { Dec. }\end{array}$ & $\begin{array}{l}1997 \\
\operatorname{Jan} .\end{array}$ & $\begin{array}{l}1997 \\
\text { Feb. }\end{array}$ & $\begin{array}{l}1997 \\
\text { Mar. }\end{array}$ & $\begin{array}{l}1097 \\
\text { IOTAL Mcf }\end{array}$ & $\begin{array}{c}\text { YTD } \\
\text { IOTAL Mcf }\end{array}$ \\
\hline 17.5 & $\begin{array}{l}14.7 \\
2.30 \\
1.45 \\
0.85 \\
0.00\end{array}$ & $\begin{array}{l}15.0 \\
2.32 \\
1.45 \\
0.87 \\
0.00\end{array}$ & $\begin{array}{l}14.3 \\
2.30 \\
1.45 \\
0.85 \\
0.00\end{array}$ & $\begin{array}{l}15.0 \\
2.32 \\
1.45 \\
0.87 \\
0.00\end{array}$ & $\begin{array}{l}14.8 \\
2.32 \\
1.45 \\
0.87 \\
0.00\end{array}$ & $\begin{array}{l}14.0 \\
2.29 \\
1.45 \\
0.84 \\
0.00\end{array}$ & $\begin{array}{l}14.7 \\
2.32 \\
1.45 \\
0.87 \\
0.00\end{array}$ & $\begin{array}{l}14.4 \\
2.37 \\
1.53 \\
0.84 \\
0.00\end{array}$ & $\begin{array}{l}15.9 \\
2.40 \\
1.53 \\
0.87 \\
0.00\end{array}$ & $\begin{array}{l}15.3 \\
2.40 \\
1.53 \\
0.87 \\
0.00\end{array}$ & $\begin{array}{l}16.0 \\
2.38 \\
1.53 \\
0.85 \\
0.00\end{array}$ & $\begin{array}{l}15.7 \\
2.40 \\
1.53 \\
0.87 \\
0.00\end{array}$ & $1,412,923$ & $1,412,923$ \\
\hline 6.4 & $\begin{array}{r}6.4 \\
3.30 \\
2.13 \\
1.17 \\
0.00\end{array}$ & $\begin{array}{r}6.4 \\
3.39 \\
2.22 \\
1.17 \\
0.00\end{array}$ & $\begin{array}{r}6.4 \\
3.38 \\
2.21 \\
1.17 \\
0.00\end{array}$ & $\begin{array}{r}6.2 \\
3.20 \\
2.03 \\
1.17 \\
0.00\end{array}$ & $\begin{array}{r}6.4 \\
2.93 \\
1.76 \\
1.17 \\
0.00\end{array}$ & $\begin{array}{r}6.4 \\
2.48 \\
1.31 \\
1.17 \\
0.00\end{array}$ & $\begin{array}{r}6.4 \\
2.61 \\
1.44 \\
1.17 \\
0.00\end{array}$ & $\begin{array}{r}6.4 \\
3.35 \\
2.18 \\
1.17 \\
0.00\end{array}$ & $\begin{array}{r}6.4 \\
4.44 \\
3.27 \\
1.17 \\
0.00\end{array}$ & $\begin{array}{r}6.4 \\
4.82 \\
3.65 \\
1.17 \\
0.00\end{array}$ & $\begin{array}{r}6.9 \\
3.44 \\
2.27 \\
1.17 \\
0.00\end{array}$ & $\begin{array}{r}6.4 \\
2.45 \\
1.28 \\
1.17 \\
0.00\end{array}$ & 591,008 & 591,008 \\
\hline 8.6 & $\begin{array}{r}8.6 \\
2.74 \\
1.80 \\
0.94 \\
0.00\end{array}$ & $\begin{array}{r}8.6 \\
2.67 \\
1.72 \\
0.95 \\
0.00\end{array}$ & $\begin{array}{r}8.5 \\
2.60 \\
1.64 \\
0.96 \\
0.00\end{array}$ & $\begin{array}{r}8.5 \\
2.57 \\
1.62 \\
0.95 \\
0.00\end{array}$ & $\begin{array}{r}8.6 \\
2.44 \\
1.50 \\
0.94 \\
0.00\end{array}$ & $\begin{array}{r}8.6 \\
2.27 \\
1.30 \\
0.97 \\
0.00\end{array}$ & $\begin{array}{r}8.6 \\
2.38 \\
1.40 \\
0.98 \\
0.00\end{array}$ & $\begin{array}{r}8.6 \\
3.28 \\
2.27 \\
1.01 \\
0.00\end{array}$ & $\begin{array}{r}8.6 \\
3.86 \\
2.87 \\
0.99 \\
0.00\end{array}$ & $\begin{array}{r}8.6 \\
3.89 \\
2.90 \\
0.99 \\
0.00\end{array}$ & $\begin{array}{r}8.3 \\
3.37 \\
2.39 \\
0.98 \\
0.00\end{array}$ & $\begin{array}{r}8.6 \\
2.62 \\
1.66 \\
0.96 \\
0.00\end{array}$ & 763,624 & 763,624 \\
\hline 35.0 & $\begin{array}{l}35.0 \\
3.17 \\
2.19 \\
0.98 \\
0.00\end{array}$ & $\begin{array}{l}35.0 \\
2.91 \\
1.97 \\
0.94 \\
0.00\end{array}$ & $\begin{array}{l}35.0 \\
3.02 \\
2.05 \\
0.97 \\
0.00\end{array}$ & $\begin{array}{l}34.7 \\
3.21 \\
2.26 \\
0.95 \\
0.00\end{array}$ & $\begin{array}{l}34.8 \\
2.92 \\
1.98 \\
0.94 \\
0.00\end{array}$ & $\begin{array}{l}34.9 \\
2.53 \\
1.56 \\
0.97 \\
0.00\end{array}$ & $\begin{array}{l}34.9 \\
2.57 \\
1.62 \\
0.95 \\
0.00\end{array}$ & $\begin{array}{l}35.0 \\
3.70 \\
2.71 \\
0.99 \\
0.00\end{array}$ & $\begin{array}{l}35.0 \\
4.69 \\
3.75 \\
0.94 \\
0.00\end{array}$ & $\begin{array}{l}34.9 \\
4.82 \\
3.86 \\
0.96 \\
0.00\end{array}$ & $\begin{array}{l}34.8 \\
3.93 \\
2.87 \\
1.06 \\
0.00\end{array}$ & $\begin{array}{l}34.7 \\
2.55 \\
1.61 \\
0.94 \\
0.00\end{array}$ & $3,134,153$ & $3,134,153$ \\
\hline 92.5 & $\begin{array}{l}90.2 \\
2.82 \\
1.91 \\
0.91 \\
0.00\end{array}$ & $\begin{array}{l}92.5 \\
2.75 \\
1.82 \\
0.93 \\
0.00\end{array}$ & $\begin{array}{l}90.2 \\
2.68 \\
1.74 \\
0.94 \\
0.00\end{array}$ & $\begin{array}{l}92.5 \\
2.64 \\
1.71 \\
0.93 \\
0.00\end{array}$ & $\begin{array}{l}92.5 \\
2.51 \\
1.59 \\
0.92 \\
0.00\end{array}$ & $\begin{array}{l}91.1 \\
2.33 \\
1.38 \\
0.95 \\
0.00\end{array}$ & $\begin{array}{l}92.5 \\
2.45 \\
1.49 \\
0.96 \\
0.00\end{array}$ & $\begin{array}{l}92.5 \\
3.36 \\
2.37 \\
0.99 \\
0.00\end{array}$ & $\begin{array}{l}92.5 \\
3.97 \\
3.00 \\
0.97 \\
0.00\end{array}$ & $\begin{array}{l}92.5 \\
4.00 \\
3.03 \\
0.97 \\
0.00\end{array}$ & $\begin{array}{l}81.2 \\
3.45 \\
2.50 \\
0.95 \\
0.00\end{array}$ & $\begin{array}{l}92.5 \\
2.70 \\
1.76 \\
0.94 \\
0.00\end{array}$ & $8,009,332$ & $8,009,332$ \\
\hline
\end{tabular}




\section{VOLUME AND PRICE REPORT}

Long-Term Imports

Volumes (MMcf/d) \& Prices (\$MMBTU) of Natural

Gas Imported During the Past 12 Months 04/01/96 - 03/31/97

Long-Term importer

Auth
Vols.

1996

99619961996

BROOKLYN NAVY YARD COGEN. PARTNERS, L.P.

Crestar Energy)

AVG Daily Quantity

Total Price (Waddington, NY)

10.0

Commodity Component

Demand Component

Reservation Fee Component

$\begin{array}{rrr}0.0 & 0.0 & 0.0 \\ \frac{0.00}{0.00} & \frac{0.00}{0.00} & 0 \\ 0.00 & 0.00 & 0.00 \\ 0.00 & 0.00 & 0.00\end{array}$

BROOKLYN NAVY YARD COGEN. PARTNERS, L.P.

(PanCanadian Petroleum Ltd.)

AVG Daily Quantity

Total Price (Waddington, NY)

Commodity Component

Demand Component

Reservation Fee Component

$\begin{array}{rrrr}0.0 & 0.0 & 0.0 & 0.0 \\ \frac{0.00}{0.00} & \frac{0.00}{0.00} & \frac{0.00}{0.00} & \frac{0.00}{0.00} \\ 0.00 & 0.00 & 0.00 & 0.00 \\ 0.00 & 0.00 & 0.00 & 0.00\end{array}$

$\begin{array}{llll}0.00 & 0.00 & 0.00 \quad 0.00\end{array}$

BROOKLYN UNION GAS COMPANY

(Alberta NE Gas (AEC Oil \& Gas Company))

AVG Daily Quantity

4.0

\begin{tabular}{rrrr}
4.0 & 4.0 & 4.0 & 4.0 \\
2.74 & 2.67 & 2.60 & 2.56 \\
\hline 1.83 & 1.74 & 1.67 & 1.63 \\
0.91 & 0.93 & 0.93 & 0.93
\end{tabular}

Total Price (Waddington, NY)

Commodity Component

Demand Component

Reservation Fee Component

$0.00 \quad 0.00$

\section{BROOKLYN UNION GAS COMPANY}

(Alberta NE Gas (TCGS Ltd. 1))

AVG Daily Quantity

Total Price (Waddington, NY)

Commodity Component

Demand Component

Reservation Fee Component

$\begin{array}{ll}24.0 & 24 \\ 2.74 & 2 \\ 1.80 & 1 \\ 0.94 & 0.9 \\ 0.00 & 0.0\end{array}$

24.0
2.67
1.72
0.95
0.00

23.9

$\frac{2.60}{1.64}$

1.64
0.96

$\frac{2.57}{1.62}$

BROOKLYN UNION GAS COMPANY

(Alberta NE Gas (TCGS Ltd. 2))

AVG Daily Quantity

\begin{tabular}{|c|c|}
\hline $\begin{array}{l}18.0 \\
2.74 \\
1.80 \\
0.94 \\
0.00\end{array}$ & $\begin{array}{l}15.8 \\
2.67 \\
1.72 \\
0.95 \\
0.00\end{array}$ \\
\hline
\end{tabular}

Total Price (Waddington, NY)

Commodity Component

Demand Component

Resenvation Fee Component

$\begin{array}{rrr}0.0 & 0.0 & 16.2 \\ 0.00 & \frac{0.00}{0.00} & \frac{2.33}{1.56} \\ 0.00 & 0.00 & 0.77 \\ 0.00 & 0.00 & 0.77\end{array}$

16.4

16.5

$\frac{3.08}{2.31} \quad \frac{4.22}{3.46}$

0.00

00

0.00

0.00

15.2

$\begin{array}{lrr}4.0 & 4.0 & 4.0\end{array}$

24.0
2.57

$\frac{2.44}{1.52}$

1.52

0.00

24.0
2.44
1.50
0.94
0.00

$24.0 \quad 24.0$

$\frac{2.27}{1.30} \quad \frac{2.38}{1.40}$

$0.97 \quad 0.98$

4.0
$\frac{3.27}{2.32}$
0.95

4.0

4.0

$\begin{array}{r}4.0 \\ 3.37 \\ \hline\end{array}$

1.43

0.95

4.0

$\frac{2.63}{1.65}$

0.65

0.98
0.00

1097

IOTAL Mcf

909,639

$1,364,408$

355,860

0.00

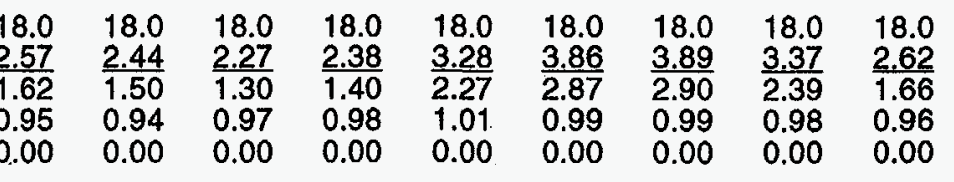

909,639

$1,364,408$

355,860

YTD

TOTAL MCI

$2,160,000$

$, 620,000$ 
VOLUME AND PRICE REPORT

Long-Term Imports

Volumes (MMct/d) \& Prices (\$MMBTU) of Natural Gas Imported During the Past 12 Months 04/01/96 - 03/31/97
Long-Term Importer

BROOKL YN UNION GAS COMPANY

(Alberta Northeast Gas (ProGas))

AVG Daily Quantity

Total Price (Waddington. NY)

Commodity Component

Demand Component

Reservation Fee Component

BROOKLYN UNION GAS COMPANY

(Alberta Northeast Gas (Producers Marketing))

AVG Daily Quantity

Total Price (Waddington. NY)

Commodity Component

Demand Component

Reservation Fee Component

CASCADE NATURAL GAS COPORATION

(IGI Resources, Inc.)

AVG Daily Quantity

Total Price (Eastport, Idaho)

Commodity Component

Demand Component

Reservation Fee Component

CASCADE NATURAL GAS CORPORATION (Mobil Oil Canada, Ltd.)

AVG Daily Quantity

Total Price (Sumas, Washington)

Commodity Component

Demand Component

Reservation Fee Component

CASCADE NATURAL GAS CORPORATION (Westcoast Gas Services)

AVG Daily Quantity

Total Price (Eastport, Idaho)

Commodity Component

Demand Component

Reservation Fee Component
Auth

$\begin{array}{lllllllll}\text { Auth. } & 1996 & 1996 & 1996 & 1996 & 1996 & 1996 & 1996 & 1996\end{array}$

$\begin{array}{lll}1996 & 1996 & 1996 \\ \text { Apr. } & \text { May. Jun. }\end{array}$

1996
Jull.

Aug.

Sep.

Nov.

$1996 \quad 1997$

1997
Feb.

997

15.8

$\begin{array}{lll}15.8 & 15.8 & 15.8 \\ 2.82 & 2.76 & 2.69 \\ 1.82 & 1.77 & 1.69 \\ 1.00 & 0.99 & 1.00 \\ 0.00 & 0.00 & 0.00\end{array}$

5.8
.69
.69
.00

15.8
2.65
1.66
0.99

15.8

15.8

15.8

$\begin{array}{ll}1.66 & 1.53\end{array}$

$\begin{array}{lll}0.00 & 0.00 & 0.00\end{array}$

$\begin{array}{ll}0.00 & 0.00\end{array}$

$\frac{2.34}{1.35}$

$\frac{2.47}{1.46}$

15.8

$\frac{3.16}{2.14}$

15.8
3.71

0.99
0.00

0.00

0.00

2.71
1.00

15.8

$\frac{4.00}{2.99}$

15.8
$\frac{3.25}{2.25}$

15.8

$\frac{2.53}{1.54}$

1.54
0.99

$\begin{array}{lll}0.00 & 0.00 & 0.00\end{array}$

8.2

\begin{tabular}{rrr}
8.2 & 8.2 & 8.2 \\
2.74 & $\frac{2.67}{1.72}$ & $\frac{2.58}{1.62}$ \\
\hline 1.80 & 1.72 & 1.94 \\
0.94 & 0.95 & 0.96
\end{tabular}

8.2
2.56
1.61
0.95
0.00

8.2
2.44
1.49

$\frac{2.44}{1.49}$

8.2
2.26

8.2
2.38
1.42

8.2
$\frac{3.28}{2.30}$

$\begin{array}{r}8.2 \\ 3.86 \\ \hline\end{array}$

0.95

0.95

0.96

0.00

$0.00 \quad 0.00$

7.4

$\begin{array}{rrr}0.0 & 0.0 & 0.0 \\ \frac{0.00}{0.00} & \underline{0.00} & 0.00 \\ 0.00 & 0.00 & 0.00 \\ 0.00 & 0.00 & 0.00\end{array}$

$\begin{array}{ll}0.0 & 0.0\end{array}$

$\underline{0.00} \quad \underline{0.00}$

0.00

0.00

0.00

0.00

0.00

12.0

\begin{tabular}{lll}
11.6 & 10.9 & 10.7 \\
1.01 & 1.01 & $\frac{0.98}{0.30}$ \\
\hline 0.33 & 0.33 & \\
0.60 & 0.60 & 0.60 \\
0.08 & 0.08 & 0.08
\end{tabular}

11.5
1.05
0.36
0.60
0.09

11.5
1.10
0.41
0.60
0.09

\begin{tabular}{l}
11.5 \\
1.15 \\
\hline 0.41 \\
0.64 \\
0.10
\end{tabular}

11.5
1.15
0.43
0.62
0.10

\begin{tabular}{l}
11.5 \\
2.41 \\
\hline 1.55 \\
0.65 \\
0.21
\end{tabular}

11.5
4.25
3.23
0.67
0.35

\begin{tabular}{l}
11.4 \\
4.53 \\
\hline 3.51 \\
0.64 \\
0.38
\end{tabular}

11.4
2.58
1.74
0.63

11.0

$\frac{1.16}{0.42}$

0.64

0.10

33.2

\begin{tabular}{|c|c|}
\hline $\begin{array}{l}24.9 \\
1.40 \\
0.99 \\
0.30 \\
0.11\end{array}$ & $\begin{array}{l}19.8 \\
1.45 \\
0.95 \\
0.36 \\
0.14\end{array}$ \\
\hline
\end{tabular}

\begin{tabular}{l}
24.9 \\
1.27 \\
\hline 0.88 \\
0.28 \\
0.11
\end{tabular}

\begin{tabular}{l}
25.5 \\
1.31 \\
\hline 0.92 \\
0.28
\end{tabular}

25.8
1.30
0.91
0.28

\begin{tabular}{l}
25.5 \\
1.32 \\
\hline 0.93 \\
0.28
\end{tabular}

24.3
2.16
1.74
0.31

26.3
$\frac{3.04}{2.67}$
0.27
0.10

\begin{tabular}{l}
26.5 \\
3.45 \\
\hline 3.07 \\
0.28
\end{tabular}

$\begin{array}{lll}0.11 & 0.10 & 0.10\end{array}$

\begin{tabular}{ll}
23.4 & 24.8 \\
2.69 & 1.61 \\
\hline 2.22 & 1.21 \\
0.35 & 0.29 \\
0.12 & 0.11
\end{tabular}
YTD

1097

TOTAL MCI

TOTALMCI

$1,425,507$

$1,425,507$

738,540

738,540

364,368

364,368

$1,015,258$

$1,015,258$

$2,243,957$

$2,243,957$ 
VOLUME AND PRICE REPORT

\section{Long-Term Imports}

Volumes (MMcf/d) \& Prices (\$MMBTU) of Natural Gas Imported During the Past 12 Months 04/01/96 - 03/31/97
Long-Term Importer

\begin{abstract}
Auth. $\quad \begin{array}{llll}1996 & 1996 & 1996 & 1996\end{array}$
\end{abstract}
Vols.

10.0

CASCADE NATURAL GAS
(Westcoast Gas Services)

AVG Daily Quantity

Total Price (Sumas, Washington)

Commodity Component

Demand Component

Reservation Fee Component

CASCADE NATURAL GAS CORPORATION (2)

(Westcoast Gas Services)

AVG Daily Quantity

Total Price (Sumas. Washington)

Commodity Component

Demand Component

Reservation Fee Component

CENTRAL HUDSON COMPANY

(Alberta NE Gas (AEC Oil \& Gas Company))

AVG Daily Quantity

Total Price (Waddíngton, NY)

Commodily Component

Demand Component

Reservation $\mathrm{Fee}$ Component

CENTRAL HUDSON COMPANY

(Alberta NE Gas (TCGS Ltd. 1))

AVG Daily Quantity

Total Price (Waddington. NY)

Commodity Component

Demand Component

Reservation Fee Component

CENTRAL HUDSON COMPANY

(Alberta NE Gas (TCGS Ltd. 2))

AVG Daily Quantity

Total Price (Waddington. NY)

Commodity Componen

Demand Component

Reservation Fee Component

\begin{tabular}{rrr}
9.8 & 9.8 & 9.8 \\
1.36 & 1.34 & 1.33 \\
\hline 0.85 & 0.85 & 0.82 \\
0.51 & 0.49 & 0.51 \\
0.00 & 0.00 & 0.00
\end{tabular}

8.8
1.42
0.87
0.55
0.00

\begin{tabular}{rrr}
4.9 & 4.9 & 4.9 \\
1.02 & $\frac{1.01}{0.91}$ & 0.95 \\
\hline 0.50 & 0.50 & 0.44 \\
0.22 & 0.21 & 0.21 \\
0.30 & 0.30 & 0.30
\end{tabular}

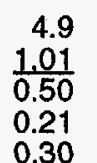

\section{1}

\begin{tabular}{|c|c|c|c|c|}
\hline $\begin{array}{r}0.1 \\
2.74 \\
1.83 \\
0.91\end{array}$ & $\begin{array}{r}0.1 \\
2.67 \\
1.74 \\
0.93\end{array}$ & $\begin{array}{r}0.1 \\
2.60 \\
1.67\end{array}$ & $\begin{array}{r}0 \\
2.5 \\
1.6 \\
0.9 \\
0.0\end{array}$ & $\frac{2}{1}$ \\
\hline
\end{tabular}

\begin{tabular}{lll}
14.1 & 14.1 & 14.0 \\
2.74 & 2.67 & 2.60 \\
\hline 1.80 & 1.72 & 1.64 \\
0.94 & 0.95 & 0.96 \\
0.00 & 0.00 & 0.00
\end{tabular}

14.1
2.57
1.62
0.95
0.00

1.5

\begin{tabular}{rrr}
1.5 & 1.3 & 1.5 \\
2.74 & 2.67 & 2.60 \\
\hline 1.80 & 1.72 & 1.64 \\
0.94 & 0.95 & 0.96 \\
0.00 & 0.00 & 0.00
\end{tabular}

\begin{tabular}{l}
1.5 \\
2.57 \\
\hline 1.62 \\
0.95 \\
0.00
\end{tabular}

\begin{tabular}{r}
9.8 \\
1.40 \\
\hline 0.91 \\
0.49 \\
0.00
\end{tabular}

\begin{tabular}{r}
9.8 \\
1.42 \\
\hline 0.91 \\
0.51 \\
0.00
\end{tabular}

\begin{tabular}{r}
9.8 \\
1.49 \\
\hline 0.99 \\
0.50 \\
0.00
\end{tabular}

9.6
2.50
1.97
0.53
0.00

9.8
$\frac{3.71}{3.21}$
0.50

$\begin{array}{r}9.8 \\ 4.30 \\ \hline 3.77\end{array}$

$\frac{4.30}{3.77}$

0.53

\begin{tabular}{rr}
9.8 & 9.8 \\
2.69 & 1.51 \\
\hline 2.11 & 0.97 \\
0.58 & 0.54
\end{tabular}

$0.00 \quad 0.00$
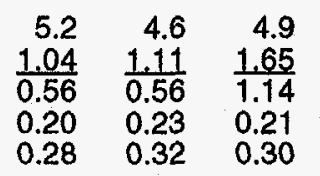

4.9
2.30
1.78
0.22
0.30

\begin{tabular}{r}
4.9 \\
3.68 \\
\hline 3.17 \\
0.21 \\
0.30
\end{tabular}

4.9
$\frac{4.33}{3.82}$

4.9
1.12

$\frac{1.12}{0.60}$

$0.25 \quad 0.22$

$0.30 \quad 0.30$

0.1
2.27
1.34
0.93

0.1
2.38
1.43
0.95
0.00

0.1
$\frac{3.27}{2.32}$
0.95
0.00

0.1
2.86
1.79
1.07

$\begin{array}{r}0.1 \\ 3.88 \\ \hline\end{array}$

$\frac{3.88}{2.94}$

0.94

$\begin{array}{r}0.1 \\ 3.37 \\ \hline 2.41\end{array}$

$\frac{3.37}{2.41}$
0.96

0.96
0.00

0.1
2.63
1.65
0.98
0.00

\begin{tabular}{l}
14.1 \\
2.44 \\
\hline 1.50 \\
0.94 \\
0.00
\end{tabular}

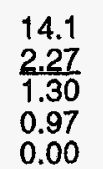

14.1
2.38
1.40
0.98
0.00

\begin{tabular}{l}
14.1 \\
3.28 \\
\hline 2.27 \\
1.01 \\
0.00
\end{tabular}

\begin{tabular}{l}
14.1 \\
3.86 \\
\hline 2.87 \\
0.99 \\
0.00
\end{tabular}

\begin{tabular}{l}
14.1 \\
3.89 \\
\hline 2.90 \\
0.99 \\
0.00
\end{tabular}

\begin{tabular}{ll}
14.1 & 14.1 \\
3.37 & 2.62 \\
\hline 2.39 & 1.66 \\
0.98 & 0.96 \\
0.00 & 0.00
\end{tabular}

$1,269,000$

$1,269,000$

1.5
2.44
1.50
0.94
0.00

1.5
2.27
1.30
0.97
0.00

1.5
2.38
1.40
0.98
0.00

\begin{tabular}{r}
1.5 \\
3.28 \\
\hline 2.27 \\
1.01 \\
0.00
\end{tabular}

1.5
3.86
2.87
0.99
0.00

\begin{tabular}{r}
1.5 \\
3.89 \\
\hline 2.90 \\
0.99 \\
0.00
\end{tabular}

$\begin{array}{lr}1.5 & 1.5 \\ \frac{3.37}{2.39} & 2.62 \\ 0.98 & 0.66 \\ 0.00 & 0.00\end{array}$

135,000

135,000

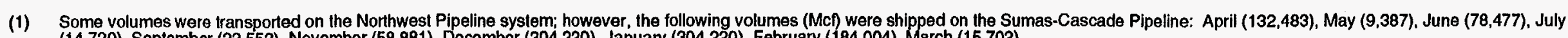

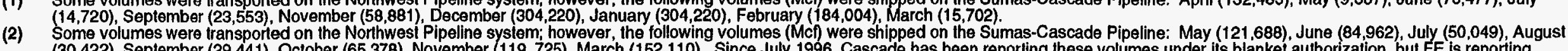

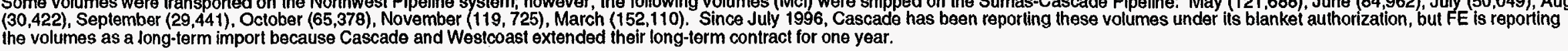


VOLUME AND PRICE REPORT

\section{Long-Term Imports}

Volumes (MMct/d) \& Prices (\$MMBTU) of Natural

Gas Imported During the Past 12 Months 04/01/96 - 03/31/97
Long-Term Importer

CENTRAL HUDSON COMPANY

(Alberta Northeast Gas (ProGas))

AVG Daily Quantity

Total Price (Waddington. NY)

Commodity Component

Demand Component

Reservation Fee Component

CENTRAL HUDSON COMPANY

(Alberta Northeast Gas (Producers Marketing))

AVG Daily Quantity

Total Price (Waddington. NY)

Commodity Component

Demand Component

Reservation Fee Component

Vols.

$19961996 \quad 1996$

Apr

1.3

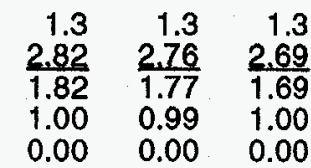

1.3
2.65
1.66
0.99
0.00

1.3
2.52
1.53

$\frac{2.52}{1.53}$

0.00

$0.00 \quad 0.00$

$\begin{array}{ll}0.99 & 0.99 \\ 0.00 & 0.00\end{array}$

$\frac{2.34}{1.35}$

1.35
0.99
0.00

$\frac{2.47}{1.46}$

1.01
0.00

$\frac{3.16}{2.14}$

1.02

$\frac{3.71}{2.71}$

1.00
0.00

4.00

$\frac{4.00}{2.99}$

1.01

$\frac{3.25}{2.25}$

1.00

2.53

1.54

0.99

3.0

$$
\begin{array}{r}
3.0 \\
2.74 \\
1.80
\end{array}
$$

3.0
2.67
1.72
0.95

3.0
2.58
1.62
0.96

0.00

CHEVRON NATURAL GAS SERVICES, INC. (1)

(Chevron Canada Pesources)

AVG Daily Quantity

Total Price (Eastport, Idaho)

Commodity Component

Demand Component

Reservation Fee Component

\begin{tabular}{rrrr}
0.0 & 0.0 & 0.0 & 13 \\
0.00 & 0.00 & 0.00 & 2 \\
\hline 0.00 & 0.00 & 0.00 & 1.8 \\
0.00 & 0.00 & 0.00 & 0.4 \\
0.00 & 0.00 & 0.00 & 0.0
\end{tabular}

74.0

\begin{tabular}{rr}
0.0 & 0.0 \\
0.00 & 0.00 \\
\hline 0.00 & 0.00 \\
0.00 & 0.00 \\
0.00 & 0.00
\end{tabular}

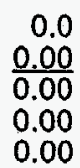

(Chevron Canada Resources)

AVG Daily Quantity

Tolal Price (Eastport. Idaho)

Commodity Component

Reservation Fee Component

CITY OF BURBANK, CALIFORNIA

(Westcoast Gas Services)

AVG Daily Quantity

Total Price (Eastpon, Idaho)

Commodity Component

Demand Component

Reservation Fee Component
4.8

\begin{tabular}{rr}
4.6 & 4.8 \\
1.56 & 1.25 \\
\hline 1.21 & 0.93 \\
0.35 & 0.32 \\
0.00 & 0.00
\end{tabular}

$0.00 \quad 0.00$

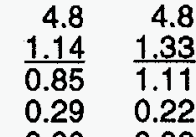

$\begin{array}{ll}0.29 & 0.22 \\ 0.00 & 0.00\end{array}$ \begin{tabular}{ll}
72.5 & 72.8 \\
0.97 & 100 \\
\hline
\end{tabular}

$\frac{3.54}{3.54} \quad \frac{1.95}{1.95}$
0.0
0.00
0.00
0.00
0.00

0.0
0.00
0.00

3.0
2.56
1.61
0.95
0.00

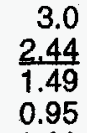

3.0

$\frac{2.26}{1.31}$

0.95

3.0
238

$\frac{2.38}{1.42}$

0.96

3.0
3.28

$\frac{3.28}{2.30}$

0.98

3.0
386

$\frac{3.86}{2.90}$

0.96

3.0
3.89

$\frac{3.89}{2.92}$

0.97

$\begin{array}{r}3.0 \\ 3.37 \\ \hline\end{array}$

$\frac{3.37}{2.41}$

0.96

$\frac{3.62}{1.67}$

1.67

0.00

268,560

268,560

$\begin{array}{lllll}3.4 & 17.0 & 17.6 & 17.6 & 17.9\end{array}$

$\frac{2.13}{1.80}$

1.80

1.80
0.33

$\frac{2.13}{1.80}$

1.80
0.33

$\frac{2.14}{1.80}$
0.34

2.25

14.3

$\frac{2.31}{1.90}$

90
.35
.00

0.41
0.00

13.0

$\frac{2.35}{1.90}$

1.90
0.45

2.7

$\frac{4.17}{1.90}$

1.90
2.27

0.0

$\frac{0.00}{0.00}$

0.00
0.00

0.00

477,718

477,718

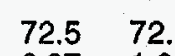

73.4

77.0

67.1

70.9

70.6

$\frac{0.98}{0.98}$

0.00

$\begin{array}{ll}0.00 & 0.00 \\ 0.00 & 0.00\end{array}$

0.00

$\frac{3.10}{3.10}$

0.00

$0.00 \quad 0.00$

0.00

0.00

$6,251,410$

$6,251,410$

422,784

422,784

(1) Supplies Hermiston cogeneration facility.
(2) Supplies are resold to Natural Gas Clearinghouse, primarily for markets in California. 
VOLUME AND PRICE REPORT

Long-Term Imports

Volumes (MMcf/d) \& Prices (\$MMBTU) of Natural

Gas Imported During the Past 12 Months 04/01/96 - 03/31/97
Demand Component

Total Price (Waddington, NY)

Commodity Componen

Reservation Fee Component

Total Price (Noves. Minnesota)
Auth

Vols.

1996

Apr. $\quad 1996$

4.1

$\begin{array}{rr}3.9 & 4 \\ 1.50 & 1.21 \\ 1.21 & 0.93 \\ 0.29 & 0.28 \\ 0.00 & 0.00\end{array}$

4.1

$\begin{array}{rrr}4.0 & 4.0 & \\ \frac{1.42}{1.21} & \frac{1.14}{0.93} & \frac{1}{0.06} \\ 0.21 & 0.21 & 0.21 \\ 0.00 & 0.00 & 0.00\end{array}$

10.0

\begin{tabular}{rr}
9.8 & 9.8 \\
3.18 & 2.38 \\
\hline 3.18 & 2.38 \\
0.00 & 0.00
\end{tabular}

$\begin{array}{ll}0.00 & 0.00\end{array}$

7.0

\begin{tabular}{rr}
0.0 & 0.0 \\
0.00 & 0.00 \\
\hline 0.00 & 0.00 \\
0.00 & 0.00 \\
0.00 & 0.00
\end{tabular}

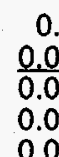

0.0
0.00
0.00
0.00
0.00

0.0

$\frac{0.00}{0.00}$

$0.00 \quad 0.00$

11.1

\begin{tabular}{rrrrr}
0.0 & 0.0 & 0.0 & 0.0 & 0.0 \\
$\underline{0.00}$ & $\underline{0.00}$ & $\underline{0.00}$ & $\underline{0.00}$ & $\underline{0.00}$ \\
\hline 0.00 & 0.00 & 0.00 & 0.00 & 0.00 \\
0.00 & 0.00 & 0.00 & 0.00 & 0.00 \\
0.00 & 0.00 & 0.00 & 0.00 & 0.00
\end{tabular}

$0.00 \quad 0.00$

$0.00 \quad 0.00$
Long-Term Importer

CITY OF GLENDALE, CALIFORNIA

(Westcoast Gas Services)

AVG Daily Quantity

Total Price (Eastpont, Idaho)

Commodity Component

Demand Component

Reservation Fee Component

CITY OF PASADENA, CALIFORNIA

(Westcoast Gas Services)

AVG Daily Quantity

Total Price (Eastport. Idaho)

Commodity Component

Reservation $\mathrm{Fe \theta}$ Component

COASTAL GAS MARKETING COMPANY (1)

(Morgan Hydrocarbons Inc.)

AVG Daily Quantity

Demand Component

COASTAL GAS MARKETING COMPANY (2)

(Morrison Petroleums Lid.)

AVG Daily Quantity

Commodity Componen

Demand Component

Reservation Fee Component

19961996

COASTAL GAS MARKETING COMPANY (3)

(Petro-Canada Resources)

AVG Daily Quantity

Reservation Fee Component

0.0
0.00

$\begin{array}{ll}0.00 & 0.00 \\ 0.00 & 0.00\end{array}$

$\begin{array}{ll}0.0 & 0.0\end{array}$

$\frac{0.00}{0.00}$

0.00
Total Price (Noyes, Minnesota)

Commodity Componen

\begin{tabular}{rrrrrrrrrr}
3.9 & 4.0 & 4.0 & 4.0 & 4.0 & 3.9 & 4.0 & 4.2 & 4.1 & 3.9 \\
1.14 & 1.16 & 1.24 & 1.22 & 1.27 & 1.55 & 1.53 & 1.54 & $\frac{1.57}{1.25}$ & $\frac{1.53}{1.25}$ \\
\hline 0.85 & 0.89 & 0.97 & 0.95 & 0.99 & 1.25 & $\frac{1.25}{1.25}$ & 0.20 & 1.25 & 0.28 \\
0.29 & 0.27 & 0.27 & 0.27 & 0.28 & 0.30 & 0.28 & 0.29 & 0.32 & 0.00 \\
0.00 & 0.00 & 0.00 & 0.00 & 0.00 & 0.00 & 0.00 & 0.00 & 0.00 & 0.00
\end{tabular}

366,734

364,400

$\frac{1.46}{1.25}$

0.21

0.21
0.00

0.00

0.22
0.00

0.21
0.00

$\frac{1.47}{1.25}$

0.22
0.00

0.00

876,735

876,735

2.65

2.65

0.00

0.00

0.65

$\frac{1.76}{1.76}$

$\frac{1.63}{1.63}$

$\begin{array}{r}9.9 \\ 3.56 \\ \hline .56\end{array}$

$3.56 \quad 5.1$

0.00

0.00 $\begin{array}{rrr}7.1 & 7.0 & 6.6 \\ \frac{3.22}{3.22} & \frac{3.85}{3.85} & \frac{2.01}{2.01} \\ 0.00 & 0.00 & 0.00\end{array}$

\begin{tabular}{rrr}
7.1 & 7.0 & 6.6 \\
$\frac{3.22}{3.22}$ & $\frac{3.85}{3.85}$ & $\frac{2.01}{2.01}$ \\
\hline .00 & 0.00 & 0.00
\end{tabular}

$0.0 \quad 7.0$

6.9
2.15
2.15

0.00

$0.00 \quad 0.00$

0.00

$\begin{array}{lll}0.00 & 0.00 & 0.00\end{array}$

621,368

621,368

0.0
0.00
0.00

10.9
219

\begin{tabular}{l}
10.9 \\
2.16 \\
\hline
\end{tabular}

\begin{tabular}{l}
11.1 \\
3.22 \\
\hline 3.22 \\
0.00
\end{tabular}

$11.1 \quad 10.4$

\begin{tabular}{l}
10.4 \\
2.01 \\
\hline
\end{tabular}

$\frac{2.01}{2.01}$

0.00

978,791

1) Supplies will serve CGM's markets in the U.S. northeast, including CGM's Eagle Point Refinery in Westville, New Jersey.

(2) Supplies sold to ProGold L.L.C. for ils corn milling facilities in North Dakota. 


\section{VOLUME AND PRICE REPORT}

\section{Long-Term Imports}

Volumes (MMct/d) \& Prices (\$MMBTU) of Natural

Gas Imported During the Past 12 Months 04/01/96 - 03/31/97

Long-Term Importer

COGEN ENERGY TECHNOLOGY L.P. (1)

(Shell Canada Limited)

AVG Daily Quantity

Total Price (Niagara Falls, NY)

Commodity Componen

Demand Component

Peservation Fee Component

COLONIAL NATURAL GAS

(Alberta NE Gas (TCGS Ltd. 1))

AVG Daily Quantity

Total Price (Waddington. NY)

Commodity Component

Demand Component

COMMONWEALTH GAS COMPANY

(Alberta NE Gas (TCGS Ltd. 1))

AVG Daily Quantity

Total Price (Waddington, NY)

Commodity Componen

Reservation Fee Component

CONNECTICUT NATURAL GAS

(Alberta NE Gas (AEC Oil \& Gas Company))

AVG Daily Quantity

Total Price (Waddington. NY)

Commodity Component

Demand Component

Reservation Fee Component

CONNECTICUT NATURAL GAS

(Alberta NE Gas (TCGS Ltd. 1))

AVG Daily Quantity

Total Price (Waddington. NY)

Commodity Component

Demand Component

Reservation Fee Component
Reservation Fee Component

Demand Component
……

Vols.

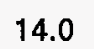

$$
\begin{array}{ll}
12.1 & 10.9 \\
2.97 & 2.97 \\
\hline 2.07 & 2.08 \\
0.90 & 0.89 \\
0.00 & 0.00
\end{array}
$$

10.6
$\frac{2.97}{2.08}$
0.89
0.00

11.1
2.97
2.08
0.89
0.00

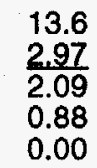

11.0
2.97
2.05
0.92
0.00

11.9
2.97
2.07
0.90
0.00

11.7
2.97
2.03
0.94
0.00

14.4
2.97

$\frac{2.97}{2.08}$

0.89
0.00

6.0

$\begin{array}{rrrrr}6.0 & 6.0 & 6.0 & 6.0 \\ 2.74 & 2.67 & 2.60 & 2.57 & 2.72 \\ 1.80 & 1.72 & 1.64 & 1.62 & 1.5 \\ 0.94 & 0.95 & 0.96 & 0.95 & 0.9 \\ 0.00 & 0.00 & 0.00 & 0.00 & 0.00\end{array}$

6.0
2.44
1.50
0.94
0.00

6.0
2.27
1.30
0.97

6.0
3.8

$\frac{2.38}{1.40}$

1.40
0.98

6.0
3.28
2.27
1.01

6.0
3.86

$\frac{3.86}{2.87}$

0.99

0.99
0.00

4.5

$\begin{array}{rrr}4.5 & 4.5 & 4.5 \\ 2.74 & 2.67 & 2.60 \\ 1.80 & 1.72 & 1.64 \\ 0.94 & 0.95 & 0.96 \\ 0.00 & 0.00 & 0.00\end{array}$

4.5
2.57
1.62
0.95
0.00

4.5
2.44
1.50
0.94
0.00

4.5

2.27
1.30

1.30
0.97

4.5

$\frac{2.38}{1.40}$

1.40
0.98

0.98
0.00

4.5

$\frac{3.28}{2.27}$

2.27
1.01

1.01
0.00

4.5

$\frac{3.86}{2.87}$

2.87
0.99

0.00 .

4.5

$\frac{3.89}{2.90}$

2.90
0.99

0.00

4.5
3.57

$\frac{3.37}{2.39}$

0.98
0.00

2.62

1.66
0.96

0.00

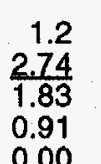

1.2
2.67
1.74
0.93
0.00

1.2
2.60
1.67
0.93
0.00

1.2
2.56
1.63
0.93
0.00

1.2
2.44
1.52
0.92
0.00

1.2
2.27
1.34
0.93
0.00

1.2
2.38
1.43
0.95
0.00

1.2
3.27
2.32
0.95
0.00

\begin{tabular}{r}
1.2 \\
3.86 \\
\hline 2.79 \\
1.07 \\
0.00
\end{tabular}

\begin{tabular}{r}
1.2 \\
3.88 \\
\hline 2.94 \\
0.94 \\
0.00
\end{tabular}

1.2
$\frac{3.37}{2.41}$
0.96
0.00

1.2
2.63
1.65
0.98
0.00

11.5

\begin{tabular}{lll}
11.5 & 11.5 & 11.4 \\
2.74 & 2.67 & 2.60 \\
\hline 1.80 & 1.72 & 1.64 \\
0.94 & 0.95 & 0.96 \\
0.00 & 0.00 & 0.00
\end{tabular}

11.5
2.57
1.62
0.95
0.00

$11.5 \quad 11.5$

$\begin{array}{ll}11.5 & 11.5 \\ 1.50 & 2.27\end{array}$

$11.5 \quad 11.5$

$\begin{array}{r}11.5 \\ 3.28 \\ \hline 2.27\end{array}$

$\frac{3.28}{2.27}$

1.40

0.98

0.94
0.00
$1 Q 97$

IOTAL MCf

$1,304,117$

404,047

108,540

108,540

(1) Gas formerly imported by Salmon Resources Lid. Supplies fuel a cogeneration facility at Castlelon-on-Hudson, N.Y. 
VOLUME AND PRICE REPORT

\section{Long-Term Imports}

Volumes (MMct/d) \& Prices (\$MMBTU) of Natural Gas Imported During the Past 12 Months 04/01/96 - 03/31/97

Leng-Term Importer

CONNECTICUT NATURAL GAS

(Alberta NE Gas (TCGS Litd. 2))

AVG Daily Quantity

Total Price (Waddington, NY)

Commodity Component

Demand Component

Reservation Fee Component

CONNECTICUT NATURAL GAS

(Alberta Northeast Gas (ProGas))

AVG Daily Quantity

Total Price (Waddington. NY)

Commodity Component

Demand Component

Reservation Fee Component

CONNECTICUT NATURAL GAS

(Alberta Northeast Gas (Producers Marketing))

AVG Daily Quantity

Total Price (Waddington. NY)

Commodity Component

Demand Component

Reservation Fee Component

CONSOLIDATED EDISON COMPANY OF N.Y.

(Alberta NE Gas (AEC Oil \& Gas))

AVG Daily

Total Price (Waddington. NY)

Commodity Component

Demand Component

Reservation Fee Component

CONSOLIDATED EDISON COMPANY OF N.Y.

(Alberta NE Gas (ProGas))

AVG Daily Quantity

Total Price (Waddington, NY)

Commodity Component

Demand Component

Reservation Fee Component

\begin{tabular}{rrr}
2.4 & 2.4 & 2.4 \\
2.74 & 2.67 & 2.58 \\
\hline 1.80 & 1.72 & 1.62 \\
0.94 & 0.95 & 0.96
\end{tabular}

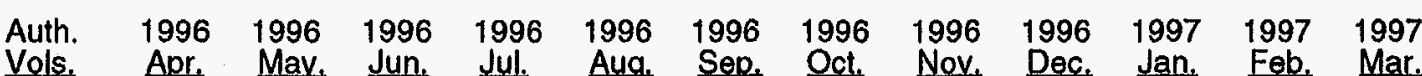

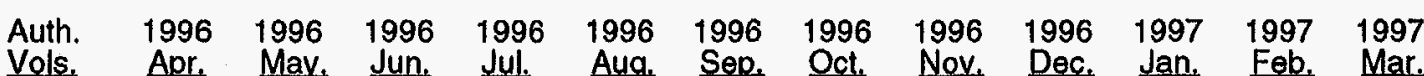

(n)

5.3

$\begin{array}{rrrr}5.3 & 4.6 & 5.3 & 5.3 \\ 2.74 & 2.67 & 2.60 & 2.57 \\ 1.80 & 1.72 & 1.64 & 1.62 \\ 0.94 & 0.95 & 0.96 & 0.95\end{array}$

$\begin{array}{llll}0.00 & 0.00 & 0.00 & 0.00\end{array}$
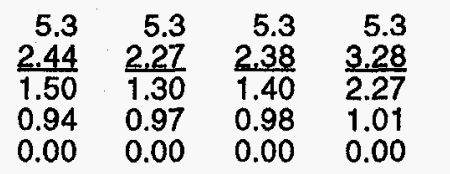

\begin{tabular}{r}
5.3 \\
3.86 \\
\hline 2.87 \\
0.99 \\
0.00
\end{tabular}
$\begin{array}{rrr}5.3 & 5.3 & 5.3\end{array}$
$\frac{3.89}{2.39} \quad \frac{2.62}{1.66}$
$0.99-0.98-1.66$
$\begin{array}{lll}0.99 & 0.98 & 0.96 \\ 0.00 & 0.00 & 0.00\end{array}$

4.6

\begin{tabular}{rrr}
4.6 & 4.6 & 4. \\
2.82 & 2.76 & 2.69 \\
\hline 1.82 & 1.77 & 1.6 \\
1.00 & 0.99 & 1.0 \\
0.00 & 0.00 & 0.00
\end{tabular}

4.6
2.65
1.66
0.99
0.00

$\begin{array}{rr}4.6 & 4.6 \\ 2.52 & 2.34 \\ 1.53 & 1.35 \\ 0.99 & 0.99 \\ 0.00 & 0.00\end{array}$

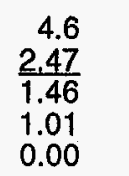

$\begin{array}{rr}4.6 & 4.6 \\ \frac{3.16}{2.14} & \frac{3.71}{2.71} \\ 1.02 & 1.00 \\ 0.00 & 0.00\end{array}$
$\begin{array}{lll}4.6 & 4.6 & 4.6\end{array}$
4.00
2.99
1.01
0.00
$\frac{3.25}{2.25}$
2.25
1.00
1.00
0.00
$\begin{array}{r}4.6 \\ 2,53 \\ \hline 1.54\end{array}$
1.54
0.99
0.00

2.4

2.4
2.56
1.61
0.95
0.00

\begin{tabular}{rr}
2.4 & 2.4 \\
2.44 & 2.26 \\
\hline 1.49 & 1.31 \\
0.95 & 0.95
\end{tabular}

$0.00 \quad 0.00$

0.00

1.1

\begin{tabular}{lr}
1.1 & 1.1 \\
2.74 & 2.67 \\
\hline 1.83 & 1.74 \\
0.91 & 0.93
\end{tabular}

\begin{tabular}{rr}
1.1 & 1.1 \\
2.60 & 2.56 \\
\hline 1.67 & 1.63 \\
0.93 & 0.93 \\
0.00 & 0.00
\end{tabular}

$\begin{array}{rr}1.1 & 1.1 \\ 2.44 & 2.27 \\ 1.52 & 1.34 \\ 0.92 & 0.93 \\ 0.00 & 0.00\end{array}$
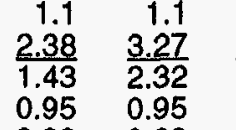

1.1
$\frac{3.86}{2.79}$
1.07

$\begin{array}{r}1.1 \\ 3.88 \\ \hline 2.94\end{array}$

$0.00 \quad 0.00$

5.3

\begin{tabular}{rrr}
5.3 & 5.3 & 5.3 \\
2.82 & 2.76 & 2.69 \\
\hline 1.82 & 1.77 & 1.69 \\
1.00 & 0.99 & 1.00 \\
0.00 & 0.00 & 0.00
\end{tabular}

\begin{tabular}{rr}
5.3 & 5.3 \\
2.52 & 2.34 \\
\hline 1.53 & 1.35 \\
0.99 & 0.99 \\
0.00 & 0.00
\end{tabular}

5.3
2.47
1.46
1.01
0.00

\begin{tabular}{r}
5.3 \\
3.16 \\
\hline 2.14 \\
1.02 \\
0.00
\end{tabular}

\begin{tabular}{r}
5.3 \\
3.71 \\
\hline 2.71 \\
1.00 \\
0.00
\end{tabular}

\begin{tabular}{r}
5.3 \\
4.00 \\
\hline 2.99 \\
1.01 \\
0.00
\end{tabular}

5.3
$\frac{3.25}{2.25}$
1.00
0.00

5.3
2.53
1.54
0.99
1097

IOTAL MCf

472,500

472,500

415,800

415,800

218,160

218,160

99,810

99,810

475,200

475,200 


\section{VOLUME AND PRICE REPORT}

\section{Long-Term Imports}

Volumes (MMcf/d) \& Prices ( $\$ / M M B T U$ ) of Natural

Gas Imported During the Past 12 Months 04/01/96 - 03/31/97

Long-Term Importer

Auth.
Vols.

\begin{tabular}{lllllll}
1996 & 1996 & 1996 & 1996 & 1996 & 1996 & 1996 \\
Apr. & May & Jun. & Jul. & Aug. & Sep & Oct \\
\hline
\end{tabular}

1996

1996

1997

$1997 \quad 1997$

1097

TOTAL MC

YTD

CONSOLIDATED EDISON COMPANY OF N.Y.

(Alberta NE Gas (Producers Marketing))

AVG Daily Quantity

$\begin{array}{rrr}2.6 & 2.6 & 2.6 \\ 2.74 & 2.67 & 2.58 \\ 1.80 & 1.72 & 1.62 \\ 0.94 & 0.95 & 0.96 \\ 0.00 & 0.00 & 0.00\end{array}$

2.6
2.56
1.61
0.95
0.00
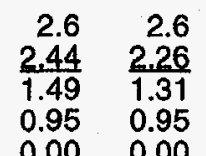

2.6
2.38
1.42
0.96

2.6
$\frac{3.28}{2.30}$
0.98

2.6
3.86

Commodity Component

Reservation Fee Component

CONSOLIDATED EDISON COMPANY OF N.Y.

(Alberta NE Gas (TCGS Ltd. 1))

AVG Daily Quantity

5.0

$\begin{array}{rrr}5.0 & 5.0 & 5.0 \\ 2.74 & 2.67 & 2.60 \\ 1.80 & 1.72 & 1.64 \\ 0.94 & 0.95 & 0.96\end{array}$

5.0
2.57
1.62
0.95

5.0
2.44
1.50

Total Price (Waddington

Demand Component

Reservation Fee Component

$0.00 \quad 0.00 \quad 0.00$

0.00

CONSOLIDATED EDISON COMPANY OF N.Y.

(Alberta NE Gas (TCGS Ltd. 2))

AVG Daily Quantity

Total Price (Waddington. NY)

Commodity Component

Demand Component

Reservation Fee Component

$\begin{array}{rrr}6.0 & 5.3 & 6.0 \\ 2.74 & 2.67 & 2.60 \\ 1.80 & 1.72 & 1.64 \\ 0.94 & 0.95 & 0.96 \\ 0.00 & 0.00 & 0.00\end{array}$

$\begin{array}{rr}6.0 & 6.0 \\ 2.57 & 2.44 \\ 1.62 & 1.50 \\ 0.95 & 0.94 \\ 0.00 & 0.00\end{array}$
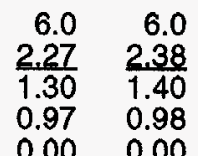

6.0
3.28
2.27
1.01
0.00

$\begin{array}{r}6.0 \\ 3.86 \\ \hline .87\end{array}$

0.00

0.00

0.00

30.6

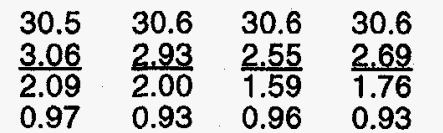

$\begin{array}{llll}2.09 & 2.00 & 1.59 & 1.76 \\ 0.97 & 0.93 & 0.96 & 0.93\end{array}$

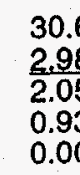

$\frac{2.98}{2.05}$

0.00

0.00

14.0

$\begin{array}{lll}14.0 & 14.0 & 14.0 \\ \frac{2.10}{2.10} & \frac{2.10}{2.10} & \frac{2.10}{2.10} \\ 0.00 & 0.00 & 0.00 \\ 0.00 & 0.00 & 0.00\end{array}$

$\begin{array}{llll}14.0 & 14.0 & 14.0 & 14.0 \\ \frac{2.20}{2.20} & \frac{2.20}{2.20} & \frac{2.20}{2.20} & \frac{2.20}{2.20} \\ 0.00 & 0.00 & 0.00 & 0.00 \\ 0.00 & 0.00 & 0.00 & 0.00\end{array}$

14.0
2.20
2.20
0.00
0.00

24.0
2.20
2.20
0.00
0.00

$\begin{array}{llll}20 & 24.0 & 24.0 & 24.0 \\ 20 & \frac{2.20}{2.20} & \frac{2.20}{2.20} & \frac{2.20}{2.20} \\ .00 & 0.00 & 0.00 & 0.00 \\ .00 & 0.00 & 0.00 & 0.00\end{array}$

234,990

234,990

444,080

444,080

$\frac{2.62}{1.66}$

0.96

0.00

(Norcen Energy Resources)

Commodity (Noyes, Minn

Reservation Fee Component 


\section{VOLUME AND PRICE REPORT} Long-Term Imports

Volumes (MMcfid) \& Prices ( $\$ M M B$ BU) of Natural

Gas Imported During the Past 12 Months 04/01/96 - 03/31/97

\section{Long-Term Importer}

CONSUMERS POWER COMPANY

(North Canadian Oils)

AVG Daily Quantity

Total Price (Noyes. Minnesota)

Commodity Component

Demand Component

Reservation Fee Component

CRESTAR ENERGY MARKETING CORPORATION (1)

(Crestar Energy)

Total Price (Noyes. Minnesota)

Commodity Component

Demand Component

Reservation Fee Component

15.0

Auth. $\quad \begin{array}{lllll}1996 & 1996 & 1996 & 1996 & 1996\end{array}$

Vols. Apr. May Jun.

14.0

$\begin{array}{lll}10.0 & 10.0 & 10 . \\ 2.10 & 2.10 & 2.10 \\ 2.10 & 2.10 & 2.10 \\ 0.00 & 0.00 & 0.00 \\ 0.00 & 0.00 & 0.00\end{array}$

$\begin{array}{ll}10.0 & \\ 2.10 & 2.8 \\ 2.10 & 2 . \\ 0.00 & 0.00 \\ 0.00 & 0.00\end{array}$

$\begin{array}{rr}9.8 & 10.0 \\ 2.20 & 2.20 \\ .20 & 2.20 \\ .00 & 0.00 \\ 0.00 & 0.00\end{array}$

10.0
2.20
2.20
0.00
0.00

\begin{tabular}{ll}
13.4 & 13.6 \\
2.47 & 2.36 \\
\hline 1.85 & 1.74 \\
0.54 & 0.54 \\
0.08 & 0.08
\end{tabular}

\begin{tabular}{lr}
10.6 & 6.4 \\
2.49 & 2.73 \\
\hline 1.77 & 1.89 \\
0.62 & 0.67 \\
0.10 & 0.17
\end{tabular}

9.9
2.54
1.82
0.61
0.11

8.0
2.14
1.37
0.66

$0.08 \quad 0.08$

0.10

(Crestar Energy)

AVG Daily Quantity

Total Price (Port of Morgan, MT)

Commodity Component

Demand Component

Reservation Fe日 Component

6.3

\begin{tabular}{rr}
0.0 & 0.0 \\
0.00 & 0.00 \\
\hline 0.00 & 0.00 \\
0.00 & 0.00
\end{tabular}

\begin{tabular}{rr}
1.3 & 6.3 \\
2.15 & 1.91 \\
\hline 1.86 & 1.63 \\
0.29 & 0.28 \\
0.00 & 0.00
\end{tabular}

0.00

0.00

\begin{tabular}{rrr}
8.9 & 7.0 & 8 \\
2.68 & 2.76 & 2.57 \\
\hline 1.66 & 1.50 & 1.54 \\
1.02 & 1.26 & 1.03 \\
0.00 & 0.00 & 0.00
\end{tabular}

\begin{tabular}{rr}
8.9 & 8.3 \\
2.57 & 2.72 \\
\hline 1.54 & 1.66 \\
1.03 & 1.06 \\
0.00 & 0.00
\end{tabular}

9.3
2.40
1.46
0.94
0.00

2.26

(Anderson Exploration Ltd.)

AVG Daily Quantity

Commodity Component

Reservation Fee Component

DARTMOUTH POWER ASSOCIATES, L.P. (4)

(BXL Energy Ltd.)

Total Price (Waddington, NY)

Commodity Component

Demand Component

Reservation Fee Component

$\begin{array}{rrr}0.0 & 0.0 & 0.0 \\ \frac{0.00}{0.00} & \frac{0.00}{0.00} & \frac{0.00}{0.00} \\ 0.00 & 0.00 & 0.00 \\ 0.00 & 0.00 & 0.00\end{array}$

\begin{tabular}{ll}
0.0 & 0.0 \\
0.00 & 0.0 \\
\hline 0.00 & 0.00 \\
0.00 & 0.00 \\
0.00 & 0.00
\end{tabular}

\begin{tabular}{rr}
0.0 & 0.0 \\
\hline 0 & 0.00 \\
\hline 0.00 & 0.00 \\
\hline 00 & 0.00 \\
\hline 0.00
\end{tabular}
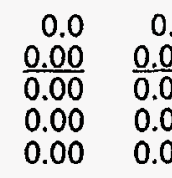

10.2
2.20
2.20
0.00
0.00

10.0
2.20
2.20
0.00
0.00

0.0
0.00
0.00
0.00

$\frac{0.00}{0.00}$

0.00
0.00

$\frac{0.00}{0.00}$

0.00

0.00

0.0

0.00

0.00

0.00

12.7

15.0
3.79
3.13
0.54
0.12

\begin{tabular}{l}
14.9 \\
4.39 \\
\hline 3.69 \\
0.58
\end{tabular}

14.9
2.99
2.34
0.56

14.4

$\frac{1.72}{1.17}$

0.49

$0.09 \quad 0.06$

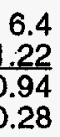

0.94

0.00

9.2
2.80
1.87
0.93
0.00

541,002

541,002

$\frac{0.00}{0.00} \quad \frac{3.25}{2.24} \quad \frac{2.80}{1.87}$

$\begin{array}{lll}0.00 & 2.24 & 1.87 \\ 0.00 & 1.01 & 0.93\end{array}$

$\begin{array}{lll}0.00 & 0.00 & 0.00\end{array}$

$1,785,036$

$1,785,036$

571,284

571,284

0

0.00

$0.00 \quad 0.00$

0.00

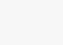

13,640

13,640

1) Supply is sold to Northern Slates Power Company for retail customers in the Grand Forks and Fargo areas of North Dakota.

2. Supply is sold to Northern States Power Company (Minnesota)

(2) Effective February 1, 1996 , Home Oil declared a parinership with Anderson Exploration Lid., and intends to carny on the business under this name.
(4) BXL Energy Ltd., as agent for Darlmouth, on occasion will supply natural gas for balancing purposes. 
VOLUME AND PRICE REPORT

\section{Long-Term Imports}

Volumes (MMct/d) \& Prices (\$MMBTU) of Natural

Gas Imported During the Past 12 Months 04/01/96 - 03/31/97

Long-Term Importer

$\begin{array}{llllllll}\text { Auth. } & 1996 & 1996 & 1996 & 1996 & 1996 & 1996 & 1996\end{array}$

DARTMOUTH POWER ASSOCIATES, L.P. (1)

(Crestar Energy Inc.)

AVG Daily Quantity

Total Price (Waddington. NY)

Commodity Component

Reservation Fee Component

DARTMOUTH POWER ASSOCIATES, L.P.

(Remington Energy Ltd.)

AVG Daily Quantity

Total Price (Waddington. NY)

Commodity Component

Demand Component

Reservation Fee Component

DISTRIGAS CORPORATION (88-37-LNG)

(Sonatrach)

AVG Daily Quantity

Total Price (Everett, Massachusetts)

Commodity Component

Demand Component

Reservation Fee Componen

DISTRIGAS CORPORATION (89-16-LNG)

(Sonatrach)

AVG Daily Quantity

Total Price (Everett. Massachusetts)

Commodity Component

Demand Component

Reservation Fee Component

EASTERN ENERGY MARKETING, INC. (2)

(Talisman Energy Inc.)

AVG Daily Quantity

Total Price (Sumas. WA)

Commodity Compone

Demand Component

Reservation Fee Component

Apr. May. Jun. Jul.
Demand Component
2.0
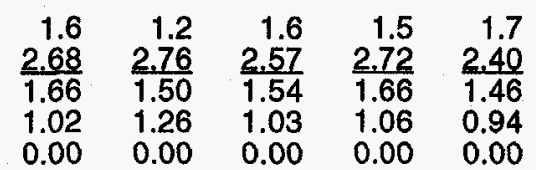

3.0

$\begin{array}{rrr}2.5 & 1.9 & 2.4 \\ 2.68 & 2.76 & 2.57 \\ 1.66 & 1.50 & 1.54 \\ 1.02 & 1.26 & 1.03 \\ 0.00 & 0.00 & 0.00\end{array}$

0.0

\begin{tabular}{lll}
86.6 & 84.2 & 0. \\
2.32 & $\frac{2.35}{2.35}$ & 0.00 \\
\hline 2.32 & 2.35 & 0.00 \\
0.00 & 0.00 & 0.00 \\
0.00 & 0.00 & 0.00
\end{tabular}

0.0

$\begin{array}{rr}0.0 & 0.0 \\ 0.00 & 0.00 \\ 0.00 & 0.00 \\ 0.00 & 0.00 \\ 0.00 & 0.00\end{array}$

$\begin{array}{rr}0.0 & 0.0 \\ 0.00 & 0.00 \\ 0.00 & 0.00 \\ 0.00 & 0.00 \\ 0.00 & 0.00\end{array}$

\begin{tabular}{r}
0.0 \\
0.00 \\
\hline 0.00 \\
0.00 \\
0.00
\end{tabular}

0.0
0.00
0.00
0.00
0.00

0.0

$\frac{0.00}{0.00}$

0.00

0.00

2.7

$\begin{array}{rrrr}0.0 & 0.0 & 0.0 & 0.0 \\ \frac{0.00}{0.00} & \frac{0.00}{0.00} & \frac{0.00}{0.00} & \frac{0.0}{0.00} \\ 0.00 & 0.00 & 0.00 & 0.00 \\ 0.00 & 0.00 & 0.00 & 0.0\end{array}$

\begin{tabular}{rr}
0.0 & 0.0 \\
0.00 & 0.00 \\
\hline 0.00 & 0.00 \\
0.00 & 0.00
\end{tabular}

$0.00 \quad 0.00$

0.00

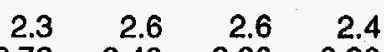

(1) Effective December 30, 1995, Ranchmen's Resources Lid. transferred all assets to Crestar Energy Inc.

(2) Supply used to fuel a 10 Megawalt gas-fired cogeneration facility near Glenns Ferry, Idaho.
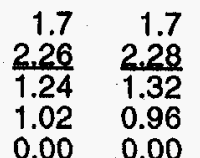
$\begin{array}{r}0.0 \\ 0.00 \\ \hline\end{array}$
0.00
0.00

1996
Dec.

1997

1997
Feb.

1997
Mar.

0.00

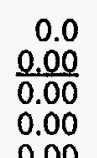

0.0
0.00
0.00

$\frac{0.00}{0.00}$

0.00

$\begin{array}{r}1.8 \\ 3.26 \\ \hline 2.25\end{array}$

1.01

0.0

$\frac{0.00}{0.00}$

0.00

$\begin{array}{r}0.0 \\ 0.00 \\ \hline 0.00\end{array}$

$\frac{0.00}{0.00}$

0.00
0.00

$\begin{array}{r}0.0 \\ 0.00 \\ \hline 0.00\end{array}$

0.00

0.00

$\begin{array}{r}2.7 \\ 3.26 \\ \hline 2.25\end{array}$

2.25
1.01

TOTALMC

$\begin{array}{ll}.00 & 0.00\end{array}$

0.00

80.5
2.72
2.72
0.00
0.00

$\begin{array}{rr}61.3 & 181.7 \\ 2.75 & 3.01\end{array}$

$2.75 \quad 3.0$

$\begin{array}{ll}.00 & 0.00\end{array}$

$\frac{0.00}{0.00}$

$\frac{0.00}{0.00}$

$\frac{0.00}{0.00}$

$\frac{0.00}{0.00}$

0.00

0.00 $\frac{2.78}{1.85}$

0.93

0.00

2.7
2.78
1.85
0.93
0.00

1097

104,946

104,946

157,477

157,477

$12,618,970$

$12,618,970$

2.71

2.71

0.00

0.00

0.00

$\frac{0.00}{0.00}$

0.00

0.00

231,824

231,824

YTD

TOTAL Maf

2.6

$\frac{1.51}{1.51}$

0.00

0.00 


\section{VOLUME AND PRICE REPORT}

\section{Long-Term Imports}

Volumes (MMct/d) \& Prices (\$/MMBTU) of Natural

Gas Imported During the Past 12 Months 04/01/96 - 03/31/97

Long-Term Importer

EASTERN ENERGY MARKETING, INC. (1)

(Talisman Energy Inc.)

AVG Daily Quantity

Total Price (Sumas, WA)

Commodity Component

Demand Component

Reservation Fee Component

\section{ELIZABETHTOWN GAS COMPANY}

(TCGS Ltd.)

AVG Daily

Total Price (Niagara Falls, NY)

Commodity Componen

Demand Component

Reservation Fee Component

ENCOGEN FOUR PARTNERS, L.P. (2)

(Sceptre Resources Limited)

AVG Daily Quantity

Total Price (Niagara Falls, NY)

Commodity Component

Demand Component

Reservation Fee Component

ENCOGEN NORTHWEST, L.P. (3)

(CanWest Gas Supply, Inc.)

AVG Daily Quantity

Total Price (Sumas, Washington)

Commodity Component

Demand Component

Reservation Fee Component

\section{ENERGYNORTH, INC}

(Alberta NE Gas (TCGS Ltd. 1))

AVG Daily Quantity

Total Price (Waddington, NY)

Commodity Component

Demand Component

Reservation Fee Component

ols. Apr May $\begin{array}{lll}1996 & 1996 & 1996\end{array}$
Auth.

$\begin{array}{llll}\text { Auth. } & 1996 \quad 1996 \quad 1996 \\ \text { Vols } & & \end{array}$

2.8

\begin{tabular}{rrr}
0.0 & 0.0 & 0.0 \\
0.00 & 0.00 & 0.00 \\
\hline 0.00 & 0.00 & 0.00 \\
0.00 & 0.00 & 0.00
\end{tabular}

$\begin{array}{ll}0.00 & 0.00 \\ 0.00 & 0.00\end{array}$

10.0

$\begin{array}{lll}10.0 & 10.0 & 10 . \\ \frac{3.13}{2.07} & \frac{3.01}{1.97} & \frac{3.0}{1.93} \\ 1.06 & 1.04 & 1.08 \\ 0.00 & 0.00 & 0.00\end{array}$

15.6

$\begin{array}{lll}15.0 & 14.6 & 15.0 \\ 2.49 & 2.51 & 2.33 \\ 1.60 & 1.63 & 1.44 \\ 0.89 & 0.88 & 0.89\end{array}$

15.0
2.72
1.87
0.85
0.00

15.0
2.67
1.82
0.85
0.00

15.0
2.81
1.92
0.89
0.00

15.0
2.45

$0.00 \quad 0.00$

00

9.6

\begin{tabular}{rr}
3.6 & 3.9 \\
2.03 & 2.03 \\
\hline 2.03 & 2.03 \\
0.00 & 0.00
\end{tabular}

$\begin{array}{ll}0.00 & 0.00 \\ 0.00\end{array}$

3.1
2.03
2.03
0.00
0.00

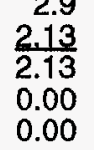

6.9
2.13
2.13
0.00

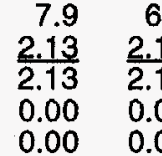

4.0

\begin{tabular}{rrr}
4.0 & 4.0 & 4.0 \\
2.74 & 2.67 & 2.60 \\
\hline 1.80 & 1.72 & 1.64 \\
0.94 & 0.95 & 0.96 \\
0.00 & 0.00 & 0.00
\end{tabular}

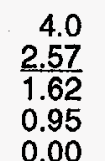

4.0
2.44
1.50
0.94
0.00

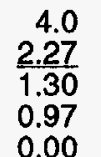

4.0
2.38
1.40
0.98
0.00

\begin{tabular}{r}
4.0 \\
3.28 \\
\hline 2.27 \\
1.01 \\
0.00
\end{tabular}

\begin{tabular}{r}
4.0 \\
3.86 \\
\hline 2.87 \\
0.99 \\
0.00
\end{tabular}

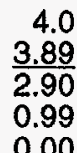

\begin{tabular}{r}
4.37 \\
\hline 2.39 \\
0.98 \\
0.00
\end{tabular}

\begin{tabular}{rr}
4.0 & 4.0 \\
3.37 & 2.62 \\
\hline 2.39 & 1.66 \\
0.98 & 0.96 \\
0.00 & 0.00
\end{tabular}

359,504

359,504

(1) Supply used to fuel a 10 Megawatl gas-fired cogeneration facility near Rupert, Idaho.

2. Supply used to fuel a 62 Megawatt gas-fired cogeneration facility located in Buffalo, New York.

(3) Supply used to fuel a 160 Megawatl gas-fired cogeneration facility located at Bellingham, Washington. 


\section{VOLUME AND PRICE REPORT}

\section{Long-Term Imports}

Volumes (MMcf/d) \& Prices (\$MMBTU) of Netural

Gas Imported During the Past 12 Months 04/01/96 - 03/31/97

Long-Term importer

ESSEX COUNTY GAS CO

(Alberta NE Gas (TCGS Ltd. 1))

AVG Daily Quantity

Total Price (Waddington, NY)

Commodity Component

Demand Component

Reservation Fee Component

FULTON COGENERATION ASSOCIATES

(Star Oil \& Gas Limited)

AVG Daily Quantity

Total Price (Niagara Falls, NY)

Commodity Component

Demand Component

Reservation Fee Component

GRANITE STATE GAS TRANSMISSION, INC.

(Direct Energy Marketing Ltd.)

AVG Daily Quantity

Total Price (North Troy, VT)

Commodity Component

Demand Component

Reservation Fee Component

GRANITE STATE GAS TRANSMISSION, INC.

(Shell Canada Limited)

AVG Daily Quantity

Total Price (North Troy. VT)

Commodity Component

Demand Component

Reservation Fee Component

Auth

Auth.
Vols.

199

Apr. 19961996

2.0

$\begin{array}{rrrr}2.0 & 2.0 & 2.0 & 2.0 \\ \frac{2.74}{1.80} & \frac{2.67}{1.72} & \frac{2.60}{1.64} & 2.5 \\ 0.94 & 0.95 & 0.96 & 0.9 \\ 0.00 & 0.00 & 0.00 & 0.00\end{array}$

6.0

$\begin{array}{rrr}5.9 & 6.0 & \\ 2.47 & 2.78 & 2 \\ 1.68 & 1.90 & 1 \\ 0.79 & 0.88 & 0.01 \\ 0.00 & 0.00 & 0.00\end{array}$

6.0

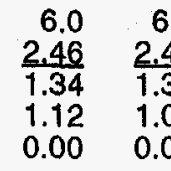

25.0

$\begin{array}{lll}25.0 & 24.8 & 24.9 \\ \frac{3.03}{1.91} & \frac{2.75}{1.64} & \frac{2.74}{1.60} \\ 1.12 & 1.11 & 1.14 \\ 0.00 & 0.00 & 0.00\end{array}$

GRANITE STATE GAS TRANSMISSION, INC (Shell Canada Limited)

AVG Daily Quantity

34.8

\begin{tabular}{llll}
34.7 & 34.6 & 34.7 & 34.2 \\
3.18 & $\frac{3.11}{2.19}$ & $\frac{2.98}{2.03}$ & $\frac{2.78}{1.87}$ \\
\hline 2.25 & 2.19 & 0.95 & 0.91 \\
0.93 & 0.92 & 0.00 & 0.00
\end{tabular}

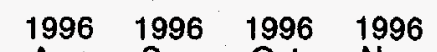

ul.

Aug.

Sep.

Qct.

Nov.

1996

Commodity Component

Reservation Fee Component

\begin{tabular}{|c|c|c|c|c|c|c|c|}
\hline $\begin{array}{r}2.0 \\
2.44 \\
1.50 \\
0.94 \\
0.00\end{array}$ & $\begin{array}{r}2.0 \\
2.27 \\
1.30 \\
0.97 \\
0.00\end{array}$ & $\begin{array}{r}2.0 \\
2.38 \\
1.40 \\
0.98 \\
0.00\end{array}$ & $\begin{array}{r}2.0 \\
3.28 \\
2.27 \\
1.01 \\
0.00\end{array}$ & $\begin{array}{r}2.0 \\
3.86 \\
2.87 \\
0.99 \\
0.00\end{array}$ & $\begin{array}{r}2.0 \\
3.89 \\
2.90 \\
0.99 \\
0.00\end{array}$ & $\begin{array}{r}2.0 \\
3.37 \\
2.39 \\
0.98 \\
0.00\end{array}$ & $\begin{array}{r}2.0 \\
2.62 \\
1.66 \\
0.96 \\
0.00\end{array}$ \\
\hline
\end{tabular}

0.00

510,768

510,768

550,170

550,170

$2.42 \quad 2.37$

$1.30 \quad 1.30$

$\begin{array}{ll}1.12 & 1.07 \\ 0.00 & 0.00\end{array}$

0.00

0.00

\begin{tabular}{|c|c|c|c|c|c|c|c|c|}
\hline $\begin{array}{l}24.7 \\
2.82 \\
1.72 \\
1.10\end{array}$ & $\begin{array}{l}24.6 \\
2.68 \\
1.58 \\
1.10 \\
0.00\end{array}$ & $\begin{array}{l}24.7 \\
2.89 \\
1.76 \\
1.13 \\
0.00\end{array}$ & $\begin{array}{l}24.8 \\
3.16 \\
2.04 \\
1.12 \\
0.00\end{array}$ & $\begin{array}{l}25.1 \\
3.40 \\
2.27 \\
1.13 \\
0.00\end{array}$ & $\begin{array}{l}25.1 \\
\frac{3.81}{2.71} \\
1.10 \\
0.00\end{array}$ & $\begin{array}{l}25.3 \\
3.16 \\
2.04 \\
1.12 \\
0.00\end{array}$ & $\begin{array}{l}25.3 \\
3.40 \\
2.27 \\
1.13 \\
0.00\end{array}$ & $\begin{array}{l}25.3 \\
3.81 \\
2.71 \\
1.10\end{array}$ \\
\hline
\end{tabular}

$2,278,620$

$3,138,501$

$34.3 \quad 34.5 \quad 34.5$

$\frac{2.64}{1.73} \frac{2.47}{1.53}$

$\begin{array}{ll}1.73 & 1.53 \\ 0.91 & 0.94\end{array}$

$\begin{array}{lll}0.00 & 0.00 & 0.00\end{array}$ $\begin{array}{llll}34.8 & 35.0 & 35.0 & 34.6\end{array}$

\begin{tabular}{ll}
3.72 & 2.60 \\
\hline 2.79 & 1.66
\end{tabular}

279

2.79
0.93

0.93
0.00

\subsection{8}

$\frac{3.18}{2.23}$

2.23
0.95 


\section{VOLUME AND PRICE REPORT}

\section{Long-Term Imports}

Volumes (MMct/d) \& Prices (\$/MMBTU) of Natural

Gas Imported During the Past 12 Months 04/01/96 - 03/31/97

Long-Term Importer

GREAT FALLS GAS COMPANY

Shell Canada Limited)

AVG Daily

Total Price (Babb. Montana)

Commodity Component

Demand Component

Reservation Fee Component

HERMISTON GENERATING COMPANY, L.P.

(CanStates Gas Marketing)

AVG Daily Quantity

Total Price (Eastport. Idaho)

Commodity Component

Demand Component

Reservation Fee Component

HERMISTON GENERATING COMPANY, L.P.

(Home Oil Company Limited)

AVG Daily Quantity

Total Price (Eastport, Idaho)

Commodity Component

Demand Component

Reservation Fee Component

$\begin{array}{lllllllllllll}\text { Auth } & 1996 & 1996 & 1996 & 1996 & 1996 & 1996 & 1996 & 1996 & 1996 & 1997 & 1997 & 1997\end{array}$

Vols. Apr. May, Jun, Jul. Aug. Sep. Oct. Nov. Dec. Jan. Feb. Mar.

5.0

\begin{tabular}{rrr}
5.0 & 5.0 & 5.0 \\
1.14 & $\frac{1.03}{1.03}$ & 0.94 \\
\hline 1.14 & 0.94 \\
0.00 & 0.00 & 0.00 \\
0.00 & 0.00 & 0.00
\end{tabular}

29.7

$\begin{array}{rrr}0.0 & 0.0 & 0.0 \\ \frac{0.00}{0.00} & \frac{0.00}{0.00} & \frac{0.00}{0.00} \\ 0.00 & 0.00 & 0.00 \\ 0.00 & 0.00 & 0.00\end{array}$

$0.00 \cdot 0.00$

15.0

$\begin{array}{rrr}0.0 & 0.0 & 0.0 \\ 0.00 & 0.00 & \frac{0.00}{0.00} \\ 0.00 & 0.00 & 0.00 \\ 0.00 & 0.00 & 0.00\end{array}$

$\begin{array}{lll}0.00 & 0.00 & 0.00\end{array}$

10.0

$\begin{array}{rrr}0.0 & 0.0 & 0.0 \\ \frac{0.00}{0.00} & \frac{0.00}{0.00} & \underline{0.0} \\ 0.00 & 0.00 & 0.00 \\ 0.00 & 0.00 & 0.00\end{array}$

$0.00 \quad 0.00$

Reservation Fee Component

INDECK ENERGY SERVICES OF OSWEGO, INC.

(Indeck Gas Supply Corp.)

AVG Daily Quantity

12.3

Total Price (Niagara Falls, NY)

Commodity Component

Demand Component

Reservation Fee Component

\begin{tabular}{rrr}
9.6 & 9.7 & 7.4 \\
2.69 & 2.69 & 2.69 \\
\hline 1.97 & 1.97 & 1.97 \\
0.72 & 0.72 & 0.72 \\
0.00 & 0.00 & 0.00
\end{tabular}

28.5
$\frac{2.68}{2.27}$
0.41
0.00
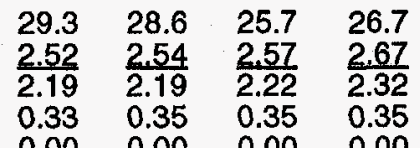

21.1
2.79
2.38
0.41

19.1
2.84
2.43

14.4
2.68
2.27
0.41

14.8
2.52
2.19
0.33

0.00

\begin{tabular}{rr}
0.0 & 0.0 \\
0.00 & 0.00 \\
\hline 0.00 & 0.00 \\
0.00 & 0.00 \\
0.00 & 0.00
\end{tabular}

$\begin{array}{r}0.0 \\ 0.00 \\ \hline 0.00\end{array}$

$\frac{0.00}{0.00}$

0.00

$\frac{0.00}{0.00}$

$0.00 \quad 0.00$

$0.00 \quad 0.00$

$\begin{array}{r}0.0 \\ 0.00 \\ \hline\end{array}$

$\frac{0.00}{0.00}$

0.00

0.00

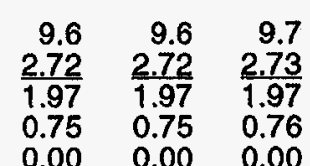

$\begin{array}{llllllllllll}1.97 & 1.97 & 1.97 & 1.97 & 1.97 & 1.97 & 1.98 & 1.98 & 1.98 & 2.03 & 2.03 & 2.03 \\ 0.72 & 0.72 & 0.72 & 0.75 & 0.75 & 0.76 & 0.77 & 0.75 & 0.77 & 0.75 & 0.74 & 0.75 \\ 0.00 & 0.00 & 0.00 & 0.00 & 0.00 & 0.00 & 0.00 & 0.00 & 0.00 & 0.00 & 0.00 & 0.00\end{array}$

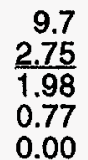

\begin{tabular}{r}
9.7 \\
2.73 \\
\hline 1.98 \\
0.75 \\
0.00
\end{tabular}

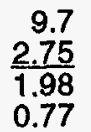

$\begin{array}{llllllllllll}1.09 & \frac{2.69}{1.97} & \frac{2.69}{1.97} & \frac{2.72}{1.97} & \frac{2.72}{1.97} & \frac{2.73}{1.97} & \frac{2.75}{1.98} & \frac{2.73}{1.98} & \frac{2.75}{1.98} & \frac{2.78}{2.03} & \frac{2.77}{2.03} & \frac{2.78}{2.03} \\ 0.72 & 0.72 & 0.72 & 0.75 & 0.75 & 0.76 & 0.77 & 0.75 & 0.77 & 0.75 & 0.74 & 0.75 \\ 0.00 & 0.00 & 0.00 & 0.00 & 0.00 & 0.00 & 0.00 & 0.00 & 0.00 & 0.00 & 0.00 & 0.00\end{array}$

$\begin{array}{llllllllllll}1.97 & 1.97 & 1.97 & 1.97 & 1.97 & 1.97 & 1.98 & 1.98 & 1.98 & 2.03 & 2.03 & 2.03 \\ 0.72 & 0.72 & 0.72 & 0.75 & 0.75 & 0.76 & 0.77 & 0.75 & 0.77 & 0.75 & 0.74 & 0.75 \\ 0.00 & 0.00 & 0.00 & 0.00 & 0.00 & 0.00 & 0.00 & 0.00 & 0.00 & 0.00 & 0.00 & 0.00\end{array}$

1097

TOTAL MCI

YTD

TOTAL MCF

447,644

447,644

$\frac{1.19}{1.19}$

1.19

0.00

0.00

703,024

703,024

0.0
0.00
0.00
0.00

0.00

355,062

355,062

$\underline{0.00}$

0.00

0.00

0.00

0.0
0.00

$\frac{0.00}{0.00}$

$\begin{array}{lll}.00 & 0.00 & 0.00 \\ .00 & 0.00 & 0.00\end{array}$ 
VOLUME AND PRICE REPORT

Long-Term Imports

Volumes (MMct/d) \& Prices (\$MMBTU) of Natural

Gas Imported During the Past 12 Months 04/01/96 - 03/31/97
Long-Term Imperter

INDECK-YERKES ENERGY SERVICES, INC.

(Indeck Gas Supply Corp.)

AVG Daily Quantity

Total Price (Niagara Falls. NY)

Commodity Component

Demand Component

Reservation Fee Component

INDECK-YERKES LTD. PARTNERSHIP (1)

(Talisman Energy Inc.)

AVG Daily Quantity

Total Price (Niagara Falls, NY)

Commodity Component

Demand Component

Reservation Fee Component

KAMINE/BESICORP BEAVER FALLS L.P.

(North Canadian Marketing Inc.)

AVG Daily Quantity

Total Price (Waddington. NY)

Commodity Component

Demand Component

Reservation Fee Component

KAMINE/BESICORP CARTHAGE L.P.

(Renaissance Energy Ltd.)

AVG Daily Quantity

Total Price (Grand Island. NY)

Commodity Component

Demand Component

Reservation Fee Component

KAMINE/BESICORP NATURAL DAM L.P.

Total Price (Waddington, NY)

Commodity Component

Demand Component

Reservation Fee Component

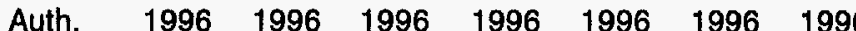

Vols.

\begin{tabular}{rrrrrrrrrrrrr}
8.9 & 9.0 & 9.0 & 9.0 & 9.0 & 9.0 & 9.2 & 9.3 & 9.2 & 9.2 & 9.2 & 9.3 \\
2.40 & 2.38 & 2.37 & 2.29 & 2.31 & 2.36 & 2.35 & 2.36 & 2.37 & 2.53 & 2.63 & 2.50 \\
\hline 1.69 & 1.66 & 1.65 & 1.58 & 1.59 & 1.63 & 1.62 & 1.64 & 1.64 & 1.81 & 1.93 & 1.78 \\
0.71 & 0.72 & 0.72 & 0.71 & 0.72 & 0.73 & 0.73 & 0.72 & 0.73 & 0.72 & 0.70 & 0.72 \\
0.00 & 0.00 & 0.00 & 0.00 & 0.00 & 0.00 & 0.00 & 0.00 & 0.00 & 0.00 & 0.00 & 0.00
\end{tabular}

0.0

$\begin{array}{rrrrrrrrrrrr}3.1 & 3.0 & 3.1 & 2.8 & 3.1 & 3.0 & 3.0 & 3.0 & 3.0 & 3.1 & 3.0 & 3.0 \\ 2.25 & 2.25 & 2.24 & 2.24 & 2.26 & 2.25 & 2.26 & 2.34 & 2.35 & 2.34 & 2.32 & 2.34 \\ 1.54 & 1.53 & 1.53 & 1.53 & 1.54 & 1.52 & 1.53 & 1.62 & 1.62 & 1.62 & 1.62 & 1.62 \\ 0.71 & 0.72 & 0.71 & 0.71 & 0.72 & 0.73 & 0.73 & 0.72 & 0.73 & 0.72 & 0.70 & 0.72 \\ 0.00 & 0.00 & 0.00 & 0.00 & 0.00 & 0.00 & 0.00 & 0.00 & 0.00 & 0.00 & 0.00 & 0.00\end{array}$

16.1

$\begin{array}{rrr}1.0 & 1.0 & 1 \\ \frac{3.23}{2.49} & \frac{3.19}{2.47} & 2.2 \\ 0.74 & 0.72 & 0.7 \\ 0.00 & 0.00 & 0.00\end{array}$

\begin{tabular}{r}
1.0 \\
3.18 \\
\hline 2.47 \\
0.71
\end{tabular}

$\begin{array}{r}0.2 \\ 7.03 \\ \hline .46\end{array}$

2.46
4.57

4.57
0.00

\begin{tabular}{r}
0.7 \\
3.52 \\
\hline 2.47 \\
1.05
\end{tabular}

1.0
3.21
2.49
0.72
0.00

1.0
$\frac{3.25}{2.53}$
0.72

$\begin{array}{r}0.9 \\ 3.65 \\ \hline\end{array}$

$\frac{3.65}{2.88}$

2.88
0.77

0.00

$\begin{array}{r}1.0 \\ 3.71 \\ \hline\end{array}$

$\frac{3.71}{2.92}$

0.79
0.00

1.0

$\frac{3.55}{2.84}$

0.71

0.00

(North Canadian Marketing Inc.)

AVG Daily Quantity

14.2

\begin{tabular}{rr}
8.6 & 8.5 \\
3.73 & 3.24 \\
\hline 2.64 & 2.13 \\
1.09 & 1.11 \\
0.00 & 0.00
\end{tabular}

\begin{tabular}{r}
7.7 \\
3.39 \\
\hline 2.17 \\
1.22 \\
0.00
\end{tabular}

\begin{tabular}{r}
7.8 \\
3.66 \\
\hline 2.47 \\
1.19
\end{tabular}

$3.66 \quad 3.32$

$0.00 \quad 0.00$

12.5

$\begin{array}{rrr}0.5 & 0.5 & 0.5 \\ \frac{3.04}{2.29} & \frac{2.99}{2.27} & \frac{3.01}{2.26} \\ 0.75 & 0.72 & 0.75 \\ 0.00 & 0.00 & 0.00\end{array}$

0.5
2.97
2.26
0.71

$0.71 \quad 0.71$

9.0
2.39
1.72
0.67

\begin{tabular}{r}
6.4 \\
3.90 \\
\hline 2.43 \\
1.47 \\
0.00
\end{tabular}

5.8
4.62$$
\frac{8.86}{4 .}
$$

\begin{tabular}{rr}
8.2 & 8.6 \\
4.00 & 2.85 \\
\hline 2.80 & 1.73 \\
1.20 & 1.12 \\
0.00 & 0.00
\end{tabular}

$0.00 \quad 0.00 \quad 0.00$

(1) There is no daily contract demand; Indeck is authorized to import 7 Bcf over eight years. The supply will be used primarily to fuel its Tonawanda Cogen facility.
$1 Q 97$

TOTAL Mcf

830,468

273,097

88,846

531,654

39,879
830,468

273,097

88,846

531,654

39,879

YTD

TOTAL MCf

.

88,846

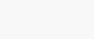




\section{VOLUME AND PRICE REPORT}

Long-Term Imports

Volumes (MMcf/d) \& Prices (\$MMBTU) of Natural

Gas Imported During the Past 12 Months 04/01/96 - 03/31/97

Long-Term Importer

Auth

KAMINE/BESICORP SOUTH GLENS FALLS L.P.

(Renaissance Energy Ltd.)

$1996 \quad 1996 \quad 1996$

1996

$1996 \quad 1996 \quad 1996$

$\begin{array}{lllll}1996 & 1996 & 1997 & 1997 & 1997\end{array}$

1097

YTD

44,427

480,534

480,534

AVG Daily Quantity

14.2

Total Price (Noyes, Minnesota)

2.90

$\frac{2.90}{2.64}$

Demand Component

Reservation Fee Component

KAMINE/BESICORP SYRACUSE L.P.

(North Canadian Marketing Inc.)

AVG Daily Quantity

Total Price (Grand Istand. NY)

Commodity Componen

Demand Component

Reservation Fee Component

\begin{tabular}{|c|c|c|c|c|c|c|c|c|c|c|c|}
\hline $\begin{array}{r}0.5 \\
3.03 \\
2.29 \\
0.74\end{array}$ & $\begin{array}{r}0.0 \\
0.00 \\
0.00 \\
0.00 \\
0.00\end{array}$ & $\begin{array}{r}0.0 \\
0.00 \\
0.00 \\
0.00 \\
0.00\end{array}$ & $\begin{array}{r}0.0 \\
0.00 \\
0.00 \\
0.00 \\
0.00\end{array}$ & $\begin{array}{r}0.0 \\
0.00 \\
0.00 \\
0.00 \\
0.00\end{array}$ & $\begin{array}{r}0.0 \\
0.00 \\
0.00 \\
0.00 \\
0.00\end{array}$ & $\begin{array}{r}0.4 \\
3.12 \\
2.26 \\
0.86 \\
0.00\end{array}$ & $\begin{array}{r}0.5 \\
3.04 \\
2.28 \\
0.76 \\
0.00\end{array}$ & $\begin{array}{r}0.5 \\
3.05 \\
2.33 \\
0.72 \\
0.00\end{array}$ & $\begin{array}{r}0.5 \\
3.69 \\
2.92 \\
0.77 \\
0.00\end{array}$ & $\begin{array}{r}0.5 \\
3.55 \\
2.76 \\
0.79 \\
0.00\end{array}$ & $\begin{array}{r}0.5 \\
3.40 \\
2.69 \\
0.71 \\
0.00\end{array}$ \\
\hline
\end{tabular}

KCS ENERGY MARKETING, INC. (1)

(Ramarro Resources Ltd.)

AVG Daily Quantity

Total Price (Niagara Falls, NY)

Commodity Component

Demand Component

Reservation Fee Component

\section{$\begin{array}{r}7.6 \\ 2.68 \\ \hline\end{array}$}

2.42

0.26

0.0
$\frac{4.38}{4.13}$

0.25

0.25

8.0
$\frac{3.01}{2.73}$
0.28

2.73
0.28

0.00

8.2
198

$\frac{1.98}{1.73}$

0.25

0.00

$\begin{array}{lll}0.00 & 0.00 & 0.00\end{array}$

0.00
0.00
0.00
0.00

0.0
0.00

$\begin{array}{r}0.0 \\ 0.00 \\ \hline 0.00\end{array}$

$\begin{array}{r}0.0 \\ 0.00 \\ \hline 0.00\end{array}$

0.0
0.00
0.00

0.0
0.00
0.00

$\begin{array}{r}0.0 \\ 0.00 \\ \hline 0.00\end{array}$

$\frac{0.00}{0.00}$

0.0
0.00
0.00

0.00

0.00
0.00

0.00
0.00

0.00
0.00

0.00
0.00

0.00
0.00

$\begin{array}{lll}0.00 & 0.00 & 0.00\end{array}$

\begin{tabular}{lll}
18.0 & 18.0 & 18.0 \\
2.92 & 2.68 & 2.7 \\
\hline 1.78 & 1.58 & 1.65 \\
1.14 & 1.10 & 1.14 \\
0.00 & 0.00 & 0.00
\end{tabular}

$18.0 \quad 18.0$

$\frac{2.65}{1.75}$

40.90

$\frac{2.49}{1.60}$

18.0

$\frac{2.33}{1.38}$

18.0
$\frac{2.52}{1.40}$
1.12
0.00

18.0
$\frac{3.05}{1.83}$
1.22
0.00

18.0
3.54
2.33
1.21

$18.0 \quad 18$.

$\frac{3.62}{2.41}$

$\frac{3.14}{1.89}$

0.00

0.00

$\begin{array}{lll}1.21 & 1.25 & 1.07\end{array}$

Reservation Fee Component

LOCKPORT ENERGY ASSOCIATES, L.P.

(ProGas Limited)

AVG Daily Quantity

Total Price (Niagara Falls, NY)

12.0

$\begin{array}{rrrr}11.0 & 9.4 & 10.2 & 8.7 \\ \frac{3.64}{2.45} & \frac{3.77}{2.43} & \frac{3.73}{2.44} & \frac{3.88}{2.44} \\ 1.04 & 1.17 & 1.12 & 1.26 \\ 0.15 & 0.17 & 0.17 & 0.18\end{array}$

\begin{tabular}{l}
10.3 \\
3.67 \\
\hline 2.44 \\
1.07 \\
0.16
\end{tabular}

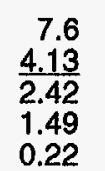

\begin{tabular}{r}
8.5 \\
3.94 \\
\hline 2.43 \\
1.32 \\
0.19
\end{tabular}

\begin{tabular}{l}
12.0 \\
3.71 \\
\hline 2.59 \\
0.97 \\
0.15
\end{tabular}

12.0
$\frac{3.66}{2.60}$
0.92

12.0
$\frac{3.68}{2.60}$
0.93

\begin{tabular}{ll}
11.2 & 11.9 \\
3.86 & 3.65 \\
\hline 2.60 & 2.59 \\
1.09 & 0.92
\end{tabular}

$\begin{array}{llll}0.92 & 0.93 & 1.09 & 0.92 \\ 0.14 & 0.15 & 0.17 & 0.14\end{array}$

Remand Component
Reservation Fee Component

$\begin{array}{llllllllllll}0.15 & 0.17 & 0.17 & 0.18 & 0.16 & 0.22 & 0.19 & 0.15 & 0.14 & 0.15 & 0.17 & 0.14\end{array}$

(1) Gas used to fuel a cageneration facility in Milford, New Jersey (formerly Energy Marketing Exchange, Inc.).

(2) Majority of supplies used to fuel cogeneralion plant at Rensselaer, N.Y.; however, the following volumes (Mcf) were surplus to its needs and went to resale market: October (431,260), November $(76,238)$, December $(101,712)$, January $(63,117)$, February $(60,401)$, March $(68,796)$. 
VOLUME AND PRICE REPORT

\section{Long-Term Imports}

Volumes (MMcfld) \& Prices (\$MMBTU) of Natural Gas Imported During the Past 12 Months 04/01/96 - 03/31/97
Long-Term Importer

LONG ISLAND LIGHTING COMPANY (Alberta NE Gas (AEC Oil \& Gas)) AVG Daily Quantity

Total Price (Waddington. NY)

Commodity Component

Demand Component.

Reservation Fee Component

LONG ISLAND LIGHTING COMPANY

(Alberta NE Gas (TCGS Ltd. 1))

AVG Daily Quantity

Total Price (Waddington. NY)

Commodity Component

Demand Component

Reservation Fee Component

LONG ISLAND LIGHTING COMPANY

(Alberta NE Gas (TCGS Ltd. 2))

AVG Daily Quantity

Total Price (Waddington, NY)

Commodity Component

Demand Componen

Reservation Fee Component

LONG ISLAND LIGHTING COMPANY

(Alberta Northeast Gas (ProGas))

AVG Daily Quantity

Total Price (Waddington, NY)

Commodity Component

Demand Component

Reservation Fee Component

LONG ISLAND LIGHTING COMPANY

(Alberta Northeast Gas (Producers Marketing))

AVG Daily Quantity

Total Price (Waddington, NY)

Commodity Component

Demand Component

Reservation Fee Component
Auth
Vols

3.1

\begin{tabular}{rrr}
3.1 & 3.1 & 3. \\
2.74 & 2.67 & 2.6 \\
\hline 1.83 & 1.74 & 1.67 \\
0.91 & 0.93 & 0.93 \\
0.00 & 0.00 & 0.00
\end{tabular}

30.4

\begin{tabular}{lll}
30.4 & 30.4 & 30.2 \\
2.74 & 2.67 & 2.60 \\
\hline 1.80 & 1.72 & 1.64 \\
0.94 & 0.95 & 0.96
\end{tabular}

$\begin{array}{lll}0.94 & 0.95 & 0.96 \\ 0.00 & 0.00 & 0.00\end{array}$

13.5

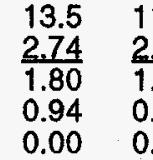

11.9

\begin{tabular}{lll}
11.9 & 11.9 & 11.9 \\
2.82 & 2.76 & 2.69 \\
\hline 1.82 & 1.77 & 1.69 \\
1.00 & 0.99 & 1.00 \\
0.00 & 0.00 & 0.00
\end{tabular}

6.2

$\begin{array}{rrr}6.2 & 6.2 & 6.2 \\ 2.74 & \frac{2.67}{1.72} & \frac{2.58}{1.62} \\ 1.80 & 1.72 & 0.96 \\ 0.94 & 0.95 & 0.00 \\ 0.00 & 0.00 & 0.00\end{array}$

0.00

$11.9 \quad 13.5$

$2.67 \quad 2.60$

$\begin{array}{ll}1.72 & 1.64 \\ 0.95 & 0.96\end{array}$

0.96
0.00
19961996

Jun.

Jul.

1a.

\begin{tabular}{lr}
1 & 3.1 \\
0 & 2.56 \\
\hline 3 & 1.63 \\
3 & 0.93 \\
0 & 0.00
\end{tabular}

3.1
2.44
1.52
0.92

0.00
1996

Sep.

1996

Oct. Nov.

Dec. Jan.

3.1
2.27
1.34
0.93

3.1
2.38
1.43
0.95

3.1
$\frac{3.27}{2.32}$
0.95

$\begin{array}{r}3.1 \\ 3.86 \\ \hline\end{array}$

$\frac{3.86}{2.79}$

2.79
1.07
0.00

$\begin{array}{r}3.1 \\ 3.88 \\ \hline\end{array}$

$\frac{3.88}{2.94}$

0.94

2.41
0.96

0.00

30.4
2.57
.62
.95
0.00

30.4

30.4

30.4

30.

1.50

1.30
0.97

$\frac{2.38}{1.40}$

1.40
0.98

$\frac{3.28}{2.27}$

30.4

30.4

30.4

$\begin{array}{lllll}1.01 & 0.99 & 2.90 & 2.39 & 1.66\end{array}$

$\begin{array}{lllll}0.00 & 0.00 & 0.00 & 0.00 & 0.00\end{array}$

13.5

$\frac{2.57}{1.62}$

0.95

0.00

13.5

$\frac{2.44}{1.50}$

0.94

0.00

13.5
2.27
1.30

$\frac{2.27}{1.30}$
0.97

13.5

$\frac{2.38}{1.40}$

13.5

3.28
2.27

13.5

$\frac{3.86}{2.87}$

0.00

0.00

0.00

0.00

\begin{tabular}{l}
13.5 \\
3.89 \\
\hline
\end{tabular}

$\frac{3.89}{2.90}$

0.99
0.00

0.98
0.00

$\begin{array}{ll}11.9 & 11.9 \\ 2.65 & 2.52 \\ 1.66 & 1.53 \\ 0.99 & 0.99 \\ 0.00 & 0.00\end{array}$

11.9

11.9

11.9

11.9

11.9

11.9

$\begin{array}{lllllll}1.35 & 1.46 & \frac{3.16}{2.14} & \frac{3.71}{2.71} & \frac{4.00}{2.99} & 2.25 & 1.54\end{array}$

$\begin{array}{lllllll}0.99 & 1.01 & 1.02 & 1.00 & 1.01 & 1.00 & 0.99 \\ 0.00 & 0.00 & 0.00 & 0.00 & 0.00 & 0.00 & 0.00\end{array}$

\begin{tabular}{rr}
6.2 & 6.2 \\
2.56 & 2.44 \\
\hline 1.61 & 1.49 \\
0.95 & 0.95 \\
0.00 & 0.00
\end{tabular}
6.2
2.26
1.31
0.95
0.00

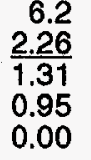

6.2
2.38
1.42
0.96
0.00 $\frac{3.28}{2.30}$

2.30
0.98

$0.00 \quad 0.00$
6.2

$\frac{3.89}{2.92}$

2.92

0.97
0.00 $\frac{3.37}{2.41}$

2.63
1.65
0.98

0.98
0.00

.00

30.4
2.62
1.66

\begin{tabular}{l}
13.5 \\
3.37 \\
\hline
\end{tabular}

2.39

13.5
2.62
1.66
0.96
0.00

11.9

$\frac{2.53}{1.54}$

$1,069,200$

0.00

1 Q97

TOTALMCI

275,850

$2,736,000$

$2,736,000$

$1,215,000$

$1,215,000$

6.2

$\frac{2.62}{1.67}$

$2.41 \quad 1.67$

$0.96,0.95$

$0.00 \quad 0.00$ 
VOLUME AND PRICE REPORT

Long-Term Imports

Volumes (MMcf/d) \& Prices (\$MMBTU) of Natural

Gas Imported During the Past 12 Months 04/01/96 - 03/31/97
Long-Term Importer

MEGAN-RACINE ASSOCIATES, INC.

(TCGS Ltd.)

AVG Daily Quantity

Total Price (Massena. New York)

Commodity Component

Demand Component

Reservation Fee Component

MICHIGAN CONSOLIDATED GAS COMPANY (1)

(TCGS Ltd.)

AVG Daily Quantity

Total Price (Noyes. Minnesota)

Commodity Component

Demand Component

Reservation Fee Component

MIDLAND COGENERATION VENTURE1

(Norcen Energy Hesources Ltd.)

AVG Daily Quantity

Total Price (Noves, Minnesota)

Commodity Componen

Demand Component

Reservation Fee Component

MIDLAND COGENERATION VENTURE2

(Shell Canada Limited)

AVG Daily Quantity

Total Price (Noves. Minnesota)

Commodity Component

Demand Component

Reservation Fee Component

MIDLAND COGENERATION VENTURE3

(Canterra Energy Limited)

AVG Daily Quantity

Total Price (Noyes. Minnesota)

Commodity Component

Demand Component

Reservation Fee Component

32.0

Auth.

$1996 \quad 1996 \quad 1996$

1996

1996

6198

996

1996

Apr. May

11.7

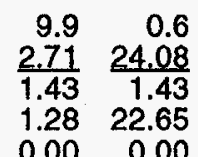

0.0
0.00
0.00
0.00
0.00

0.0
0.00
0.00
0.00
0.00

0.0

0.0

$0.00 \quad 0.00$

$\frac{0.00}{0.00}$

$\frac{0.00}{0.00}$

$\begin{array}{r}0.0 \\ 0.00 \\ \hline\end{array}$

0.0
0.00
0.00
0.00
0.00

0.3
$\frac{3.90}{1.37}$

0.00
0.00

0.00

0.00

0.5
2.72
1.37
1.35
0.00

0.4
3.42
1.92
1.50

$\frac{5.54}{1.92}$

1.92
3.62

$\frac{4.21}{1.92}$ 2.29

0.00

$\begin{array}{rrrrrrrrrrrr}30.0 & 30.0 & 30.0 & 29.6 & 30.0 & 28.9 & 30.0 & 0.0 & 0.0 & 0.0 & 0.0 & 0.0 \\ 2.51 & 2.07 & 2.19 & 2.44 & 2.18 & 1.74 & 1.72 & 0.00 & \frac{0.00}{0.00} & 0.00 & \frac{0.00}{0.00} & \frac{0.00}{0.00} \\ 2.06 & 1.62 & 1.72 & 1.98 & 1.74 & 1.24 & 1.25 & 0.00 & 0.00 & 0.00 & 0.00 & 0.00 \\ 0.45 & 0.45 & 0.47 & 0.46 & 0.44 & 0.50 & 0.47 & 0.00 & 0.00 & 0.00 & 0.00 & 0.00 \\ 0.00 & 0.00 & 0.00 & 0.00 & 0.00 & 0.00 & 0.00 & 0.00 & 0.00 & 0.00 & 0.00 & 0.00\end{array}$

10.0

$\begin{array}{lll}10.0 & 10.0 & 10.0 \\ \frac{1.80}{1.34} & \frac{1.79}{1.35} & \frac{1.80}{1.32} \\ 0.46 & 0.44 & 0.48 \\ 0.00 & 0.00 & 0.00\end{array}$

10.0
1.78
1.32
0.46
0.00

\begin{tabular}{l}
10.0 \\
1.78 \\
\hline 1.32 \\
0.46 \\
0.00
\end{tabular}

\begin{tabular}{l}
10.0 \\
1.81 \\
\hline 1.33 \\
0.48 \\
0.00
\end{tabular}

10.0
1.81
1.43
0.38
0.00

10.0
1.80
1.31

$\frac{1.80}{1.31}$

1.31
0.49
0.00

\begin{tabular}{l}
10.0 \\
1.79 \\
\hline 1.32 \\
0.47 \\
0.00
\end{tabular}

10.0
1.79
1.31
0.48
0.00

10.0
1.83
1.30

$\frac{1.83}{1.30}$

1.30
0.53
0.00

10.0

$\frac{1.78}{1.31}$

1.31
0.47

0.47
0.00

15.0

\begin{tabular}{ll}
15.0 & 15.0 \\
1.70 & $\frac{1.68}{1.25}$ \\
\hline 1.27 & 1.43 \\
0.43 & 0.43 \\
0.00 & 0.00
\end{tabular}

\begin{tabular}{l}
15.0 \\
1.70 \\
\hline 1.25 \\
0.45 \\
0.00
\end{tabular}

15.0

\begin{tabular}{r}
15.0 \\
1.72 \\
\hline 1.27 \\
0.45 \\
0.00
\end{tabular}

15.0
1.72
1.27
0.45
0.00

15.0
1.70
1.27
0.43
0.00

15.0
1.73
1.27
0.46
0.00

\begin{tabular}{l}
15.0 \\
1.71 \\
\hline 1.28 \\
0.43 \\
0.00
\end{tabular}

15.0
$\frac{1.70}{1.26}$
0.44

$15.0 \quad 15.0$

15.0
$\frac{1.70}{1.27}$

$\frac{1.7}{1.2}$

0.49

0.43

15.0

$\begin{array}{llll}15.0 & 15.0 & 15.0 & 15.0 \\ \frac{1.91}{1.46} & \frac{1.89}{1.46} & \frac{1.91}{1.46} & \frac{1.89}{1.46} \\ 0.45 & 0.43 & 0.45 & 0.43 \\ 0.00 & 0.00 & 0.00 & 0.00\end{array}$

\begin{tabular}{l}
1.5 .0 \\
1.89 \\
\hline 1.46 \\
0.43 \\
0.00
\end{tabular}

15.0
1.90
1.46
0.44
0.00

\begin{tabular}{l}
15.0 \\
1.90 \\
\hline 1.46 \\
0.44
\end{tabular}

15.0
1.91
1.45
0.46

\begin{tabular}{l}
15.0 \\
1.90 \\
\hline 1.46 \\
0.44
\end{tabular}

\begin{tabular}{l}
15.0 \\
1.90 \\
\hline 1.46 \\
0.44
\end{tabular}

\begin{tabular}{l}
15.0 \\
1.90 \\
\hline 1.41
\end{tabular}

1.41
0.49

$0.49 \quad 0.44$

$0.00 \quad 0.00$

1097

IOTAL MCf

27,365

900,000

900,000

$1,350,000$

$1,350,000$

0

YTD

TOTAL MCI

27,365

(1) This contract expired on 10/31/96. 


\section{Long-Term importer}

MIDLAND COGENERATION VENTURE4 (TransCanada Pipelines Limited)

AVG Daily Quantity

Total Price (Noyes, Minnesota)

Commodity Component

Demand Component

Reservation Fee Component

MIDLAND COGENERATION VENTURES (Poco Petroleums Ltd.)

AVG Daily Quantity

Total Price (Noyes. Minnesota)

Commodity Component

Demand Component

Reservation Fee Component

MIDLAND COGENERATION VENTUREG

(North Canadian Oils, Ltd.)

AVG Daily Quantity

Total Price (Noyes. Minnesota)

Commodity Component

Demand Component

Reservation Fee Component

MINNEGASCO, INC.

(TransCanada Pipelines Limited)

AVG Daily Quantity

Total Price (Noves, Minnesota)

Commodity Component

Demand Component

Reservation Fee Component

N.Y. STATE ELECTRIC \& GAS CO.

(Alberta NE Gas (AEC Oil \& Gas Co.)

AVG Daily Quantity

Total Price (Waddington. NY)

Commodity Component

Demand Component

Reservation Fee Component
VOLUME AND PRICE REPORT

\section{ong-Term Imports}

Volumes (MMcf/d) \& Prices (\$MMBTU) of Natural

Gas Imported During the Past 12 Months 04/01/96 - 03/31/97

Vols

Apr. May. Jun

15.0

$\begin{array}{lll}15.0 & 15.0 & 15.0 \\ \frac{1.71}{1.26} & \frac{1.69}{1.24} & \frac{1.71}{1.24} \\ 0.45 & 0.45 & 0.47 \\ 0.00 & 0.00 & 0.00\end{array}$
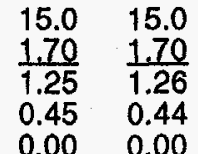

15.0

$\frac{1.71}{1.23}$

0.00

$0.00 \quad 0.00$

15.0

\begin{tabular}{lll}
15.0 & 15.0 & 15.0 \\
2.14 & 2.13 & 2.16 \\
\hline 1.70 & 1.71 & 1.72 \\
0.44 & 0.42 & 0.44
\end{tabular}

$\begin{array}{lll}0.00 & 0.00 & 0.00\end{array}$

15.0
2.15
1.73
0.42

0.42

0.00

0.00

\begin{tabular}{l}
15.0 \\
1.71 \\
\hline
\end{tabular}

$\frac{1.71}{1.24}$

1996

1996

1997

$1997 \quad 1997$

\begin{tabular}{l}
15.0 \\
1.69 \\
\hline 1.22
\end{tabular}

$\frac{1.70}{1.19}$

$1.19 \quad 1.20$

0.51

1.20
0.49

0.49

$\frac{1.74}{1.21}$

0.53

10.0

$\begin{array}{rrrr}8.3 & 8.4 & 5.8 & 1.1 \\ 2.46 & 2.43 & 2.57 & \frac{5.94}{2.21} \\ 1.71 & 1.71 & 1.50 & 2.21 \\ 0.75 & 0.72 & 1.07 & 3.73 \\ 0.00 & 0.00 & 0.00 & 0.00\end{array}$

1.1
$\frac{5.97}{2.22}$
3.75

3.75
0.00

10.2
$\frac{2.39}{1.76}$

1.76
0.63

0.00

7.5

7.5

15.0

1.76
0.88

$\frac{2.63}{1.77}$

0.86
0.00

$\frac{2.20}{1.75}$

0.45

15.0

$\frac{2.20}{1.77}$

15.0

1.77

0.43
0.00

1.77

0.44

15.0

$\frac{2.26}{1.78}$

0.48 .

50.0

$\begin{array}{lll}42.0 & 34.4 & 34 \\ 2.48 & 2.29 & 2.34 \\ 1.95 & 1.76 & 1.80 \\ 0.53 & 0.53 & 0.54 \\ 0.00 & 0.00 & 0.00\end{array}$

34.6
2.34
1.80
0.54
0.00

34.4
2.45
1.93
0.52
0.00

34.4
2.39
1.87
0.52

3.7

1.8

5.0

$\begin{array}{r}5.0 \\ 3.92 \\ \hline\end{array}$

2.23
1.12

0.00

1.69
0.00

$\frac{3.08}{2.24}$

0.84
0.00

$\frac{3.44}{2.83}$

0.80

10.0
2.79
2.14
0.65

0.65

1.3

$\begin{array}{rrr}1.3 & 1.3 & 1.3 \\ \frac{2.74}{1.83} & \frac{2.67}{1.74} & 2.60 \\ 0.91 & 0.93 & 0.93 \\ 0.00 & 0.00 & 0.00\end{array}$

\begin{tabular}{ll}
1.3 & 1.3 \\
2.56 & 2.44 \\
\hline 1.63 & 1.52 \\
0.93 & 0.92 \\
0.00 & 0.00
\end{tabular}

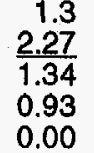

1.3
2.38
1.43
0.95
0.00

1.3
$\frac{3.27}{2.32}$
0.95
0.00

1.3
$\frac{3.86}{2.79}$
1.07
0.00

1.3
$\frac{3.88}{2.94}$
0.94
0.00

$\frac{3.37}{2.41}$

\begin{tabular}{ll}
1.3 & 1.3 \\
3.37 & 2.63 \\
\hline .41 & 1.65 \\
.96 & 0.98 \\
.00 & 0.00
\end{tabular}
1097

TOTAL Mc

0.00

7.7

$\frac{2.64}{1.81}$

0.83

0.83
0.00

10.0
2.25
1.65
0.60
0.00

YTD

TOTAL MC

$1,350,000$

$1,125,000$

$1,125,000$

900,000

900,000

$4,445,596$

$4,445,596$

$\frac{2.10}{1.50}$

1.50

0.60

.3
63
.65
.98
.00




\section{VOLUME AND PRICE REPORT}

\section{Long-Term Imports}

Volumes (MMct/d) \& Prices (\$MMBTU) of Natural

Gas Imported During the Past 12 Months 04/01/96 - 03/31/97

Long-Term Importer

N.Y. STATE ELECTRIC \& GAS CO.

(Alberta NE Gas (TCGS Ltd. 2))

AVG Daily Quantity

Total Price (Waddington. NY)

Commodity Component

Demand Component

Reservation Fee Component

N.Y. STATE ELECTRIC \& GAS CO.

(Alberta Northeast Gas (ProGas))

AVG Daily Quantity

Total Price (Waddington. NY)

Commodity Component

Demand Component

Reservation Fee Component

N.Y. STATE ELECTRIC \& GAS CO.

(Alberta Northeast Gas (Producers Marketing))

AVG Daily Quantity

Total Price (Waddington. NY)

Commodity Component

Demand Component

Reservation Fee Component

N.Y. STATE ELECTRIC \& GAS CO.

(Crestar Energy)

AVG Daily Quantity

Total Price (Grand Island. NY)

Commodity Component

Demand Component

Reservation Fee Component

N.Y. STATE ELLECTRIC \& GAS CO. (1)

(Progas Limited)

AVG Daily Quantity

Total Price (Niagara Falls, NY)

Commodity Component

Demand Component

Reservation Fee Component
Auth

Vo

6.8

$\begin{array}{rr}6.8 & 5.9 \\ 2.74 & 2.67 \\ 1.80 & 1.72 \\ 0.94 & 0.95\end{array}$

$\begin{array}{ll}0.94 & 0.95 \\ 0.00 & 0.00\end{array}$

\begin{tabular}{rr}
6.8 & 6.7 \\
2.60 & 2.57 \\
\hline 1.64 & 1.62 \\
0.96 & 0.95 \\
0.00 & 0.00
\end{tabular}

6.8
2.44
1.50
0.94
0.00

6.7
2.27
1.30
0.97
0.00

6.8
2.38
1.40

1.40

0.98

0.00

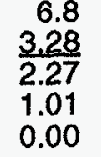

6.8
3.86
2.87
0.99

0.99
0.00

5.9

$\begin{array}{rrr}5.9 & 5.9 & 5.9 \\ 2.82 & 2.76 & 2.69 \\ 1.82 & 1.77 & 1.69 \\ 1.00 & 0.99 & 1.00 \\ 0.00 & 0.00 & 0.00\end{array}$

5.9
2.65
1.66
0.99
0.00

5.9
2.52
1.53
0.99
0.00

5.9
2.34
1.35
0.99
0.00

5.9
$\frac{2.47}{1.46}$
1.01
0.00

5.9
$\frac{3.16}{2.14}$
1.02
0.00

5.9
$\frac{3.71}{2.71}$
1.00
0.00

\begin{tabular}{r}
5.9 \\
4.00 \\
\hline 2.99 \\
1.01 \\
0.00
\end{tabular}

$5.9 \quad 5.9$

$\frac{3.25}{2.25} \quad \frac{2.53}{1.54}$

$\begin{array}{ll}1.00 & 0.99\end{array}$

$0.00 \quad 0.00$

3.0

\begin{tabular}{rrrrr}
3.0 & 3.0 & 3.0 & 3.0 & 3.0 \\
2.74 & 2.67 & 2.58 & 2.56 & 2.44 \\
\hline 1.80 & 1.72 & 1.62 & 1.61 & 1.49 \\
0.94 & 0.95 & 0.96 & 0.95 & 0.95 \\
0.00 & 0.00 & 0.00 & 0.00 & 0.00
\end{tabular}

3.0
2.26
1.31
0.95
0.00

3.0
2.38
1.42
0.96

$\begin{array}{r}3.0 \\ 3.28 \\ \hline 230\end{array}$

$\frac{3.28}{2.30}$
0.98

\begin{tabular}{r}
3.0 \\
3.86 \\
\hline 2.90 \\
0.96
\end{tabular}

$\begin{array}{r}3.0 \\ 3.89 \\ \hline 2.92\end{array}$

$\frac{3.89}{2.92}$

0.97

$\begin{array}{r}3.0 \\ 3.37 \\ \hline 2.41\end{array}$

$\frac{3.37}{2.41}$
0.96

3.0

$\frac{2.62}{1.67}$

0.95
0.00

10.0

$\begin{array}{ll}10.2 & 8.5 \\ 2.53 & 2.39 \\ 1.65 & 1.49 \\ 0.88 & 0.9 \\ 0.00 & 0.00\end{array}$

$8.5 \quad 10.3$

$\begin{array}{lr}10.3 & 9.9 \\ 2.36 & 2.45 \\ 1.46 & 1.56 \\ 0.90 & 0.89 \\ 0.00 & 0.00\end{array}$

9.9
2.44
1.55
0.89
0.00

10.3
2.29
1.38
0.91
0.00

9.9
2.35
1.44
0.91
0.00

\begin{tabular}{l}
10.2 \\
2.55 \\
\hline 1.64 \\
0.91 \\
0.00
\end{tabular}

9.9
$\frac{3.02}{2.13}$

$\begin{array}{r}9.9 \\ 3.13 \\ \hline\end{array}$

$\frac{3.13}{2.23}$

$\begin{array}{r}9.9 \\ 3.08 \\ \hline 2.09\end{array}$

$\frac{2.79}{1.63}$

1.63
1.16

$\begin{array}{llll}0.00 & 0.00 & 0.00 & 0.00\end{array}$

9.0

$\begin{array}{rrrr}8.3 & 8.0 & 7.9 & 8.2 \\ \frac{3.15}{2.16} & \frac{2.98}{2.02} & \frac{2.88}{1.86} & \frac{2.92}{1.96} \\ 0.99 & 0.96 & 1.02 & 0.96 \\ 0.00 & 0.00 & 0.00 & 0.00\end{array}$

\begin{tabular}{r}
8.2 \\
2.95 \\
\hline 2.00 \\
0.95 \\
0.00
\end{tabular}

8.0
3.00
2.00
1.00
0.00

\begin{tabular}{r}
8.1 \\
2.98 \\
\hline 2.00 \\
0.98 \\
0.00
\end{tabular}

\begin{tabular}{r}
8.1 \\
3.03 \\
\hline 2.02 \\
1.01 \\
0.00
\end{tabular}

\begin{tabular}{r}
4.5 \\
3.63 \\
\hline 1.90 \\
1.73 \\
0.00
\end{tabular}

\begin{tabular}{r}
3.8 \\
4.20 \\
\hline 2.11 \\
2.09 \\
0.00
\end{tabular}

\begin{tabular}{r}
6.8 \\
3.28 \\
\hline 1.98 \\
1.30
\end{tabular}

6.4
3.07
1.85
1.22
0.00
TOTAL Mcf

606,500

606,500

534,600

534,600

268,560

268,560

815,751

815,751

505,877

505,877

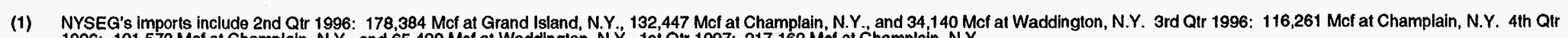
1996: 191,573 Mcf at Champlain, N.Y., and 65.420 Mcf at Waddinglon, N.Y. 1st Qtt 1997: 217,162 Mof at Champlain, N.Y. 
VOLUME AND PRICE REPORT

Long-Term Imports

Volumes (MMcf/d) \& Prices (\$MMBTU) of Natural

Gas Imported During the Past 12 Months 04/01/96 - 03/31/97
Long-Term Importer

NATIONAL FUEL GAS DISTRIBUTION CORP.

(Alberta NE Gas (TCGS Ltd.))

AVG Daily Quantity

Commodity Component

Reservation Fee Component

NATIONAL STEEL CORPORATION

(Direct Energy Marketing, Ltd.)

AVG Daily Quantity

Total Price (Detroit, Michigan)

Commodity Component

Demand Component

Reservation Fee Component

NEW ENGLAND POWER COMPANY (1)

(Canadian Natural Resources)

AVG Daily Quantity

Total Price (Waddington. NY)

Commodity Component

Demand Component

Reservation Fee Component

NEW ENGLAND POWER COMPANY

(Renaissance Energy Limited)

AVG Daily Quantity

Commodity Component

Reservation Fee Component

NEW JERSEY NATURAL GAS COMPANY

(Alberta NE Gas (AEC Oil \& Gas Company))

AVG Daily Quantity

Total Price (Waddington. NY)

Commodity Component

Demand Component

Reservation Fee Component
Total Price (Niagara Falls, NY)

Demand Component

Total Price (Waddington. NY)

Demand Component
A

Vo 10.0

$\begin{array}{llll}10.0 & 10.0 & 10.0 & 1 \\ 2.72 & 2.65 & 2.59 & 2 \\ 1.81 & 1.72 & 1.65 & 1 \\ 0.91 & 0.93 & 0.94 & 0 \\ 0.00 & 0.00 & 0.00 & 0.0\end{array}$

18.0

$\begin{array}{lll}22.7 & 17.5 & 15.5 \\ \frac{2.53}{2.53} & 2.49 & 2.36 \\ 0.00 & 0.09 & 2.36 \\ 0.00 & 0.00 & 0.00\end{array}$

45.0

\begin{tabular}{lll}
19.8 & 20.0 & 18.3 \\
2.61 & 2.25 & 2.28 \\
\hline 1.71 & 1.36 & 1.38 \\
0.90 & 0.89 & 0.90 \\
0.00 & 0.00 & 0.00
\end{tabular}

0.00

15.0

$\begin{array}{lll}13.9 & 15.0 & 16.7 \\ \frac{3.26}{2.51} & \frac{2.67}{1.93} & 2.74 \\ 0.75 & 0.74 & 0.74 \\ 0.00 & 0.00 & 0.00\end{array}$

1.9

\begin{tabular}{rrr}
1.9 & 1.9 & 1.9 \\
2.74 & 2.67 & 2.60 \\
\hline 1.83 & 1.74 & 1.67 \\
0.91 & 0.93 & 0.93 \\
0.00 & 0.00 & 0.00
\end{tabular}

$\begin{array}{llll}1996 & 1996 \quad 1996 & 1996\end{array}$

Jul.

$10.0 \quad 10.0$

$\frac{2.57}{1.62} \quad \frac{2.43}{1.51}$

$\begin{array}{ll}0.95 & 0.92 \\ 0.00 & 0.00\end{array}$

10.0
2.27

$\frac{2.27}{1.30}$

0.97
0.00

$\frac{2.37}{1.41}$

0.96
0.00

1996

Nov.

1996

Dec.

Jan.

(1)

Feb.

Mar

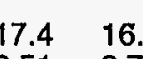

$\begin{array}{lll}17.4 & 16.5 & 13.9 \\ 2.51 & 2.76 & 2.55 \\ 2.51 & 2.76 & 2.55 \\ 0.00 & 0.00 & 0.00 \\ 0.00 & 0.00 & 0.00\end{array}$

0.00

10.0

$\frac{3.26}{2.27}$

10.0

10.0
$\frac{3.87}{2.88}$

0.99
0.00

0.00

10.0
3.89

$\frac{3.89}{2.90}$

0.99
0.00

$\begin{array}{r}7.5 \\ 3.37 \\ \hline\end{array}$

$\frac{3.37}{2.40} \quad 2.01$

$0.97 \quad 0.94$

$0.00 \quad 0.00$

$\begin{array}{ll}3.9 & 17.6 \\ .55 & 2.57 \\ .55 & 2.57 \\ .00 & 0.00 \\ 0.00 & 0.00\end{array}$

17.6

$\frac{2.60}{2.60}$

19.1
2.70

$\frac{2.70}{2.70}$

19.7
3.94

18.8
3.29

$\begin{array}{ll}3.94 & 3.29 \\ 0.00 & 0.00\end{array}$

0.00

0.00
0.00

0.00

0.00
0.00

15.6

15.6
2.67
2.67

0.00
0.00

$1,622,576$

830,000
$1,622,576$

1097
TOTAL

830,000

$\begin{array}{llllll}18.7 & 18.2 & 18.3 & 18.4 & 18.5 & 18.4\end{array}$

2.43
1.53
0.90

$\frac{2.29}{1.41}$

1.41
0.88

$\frac{2.07}{1.17}$

0.90

2.04

$\frac{2.08}{1.16}$

$90 \quad 0.92$

2.56

1.66
0.90

18.3

$\frac{2.69}{1.77}$

\begin{tabular}{l}
18.3 \\
2.65 \\
\hline
\end{tabular}

$\frac{2.65}{1.73}$

0.92
0.00

18.4

$\frac{1.88}{0.99}$

0.99
0.89

0.89
0.00

$1,651,268$

$1,651,268$

\begin{tabular}{lll}
15.1 & 16.7 & 16.7 \\
$\frac{3.06}{2.32}$ & 2.76 & 2.26 \\
\hline 0.74 & 0.74 & 1.52 \\
0.00 & 0.00 & 0.74 \\
& &
\end{tabular}

16.6

16.5
$\frac{3.00}{2.24}$

$\begin{array}{r}0.0 \\ 0.00 \\ \hline .00\end{array}$

1.50
0.75

0.76
0.00

0.00
0.00

0.0
0.00
0.00
0.00

16.5
$\frac{3.31}{2.57}$

16.6
2.22

$\frac{2.22}{1.49}$

0.73

$0.00 \quad 0.00$

976,270

976,270

0.00

(1) On December 20, 1995, OFP, in Order 551-C, amended NEP's import authorization by increasing the daily contract demand from 35 to 45 MMct/day. 
Page - 30

\section{VOLUME AND PRICE REPORT}

\section{Long-Term Imports}

Volumes (MMcf/d) \& Prices (\$/MMBTU) of Natural Gas Imported During the Past 12 Months 04/01/96 - 03/31/97

\section{Leng-Term Importer}

NEW JERSEY NATURAL GAS COMPANY

(Alberta NE Gas (TCGS Ltd. 1))

AVG Daily Quantity

Total Price (Waddington. NY)

Commodity Componen

Demand Component

Reservation Fee Component

NEW JERSEY NATURAL GAS COMPANY

(Alberta NE Gas (TCGS Ltd. 2))

AVG Daily Quantity

Total Price (Waddington. NY)

Commodity Component

Demand Component

Reservation Fee Component

NEW JERSEY NATURAL GAS COMPANY

(Alberta Northeast Gas (ProGas))

AVG Daily Quantity

Total Price (Waddington. NY)

Commodity Component

Demand Component

Reservation Fee Component

NEW JERSEY NATURAL GAS COMPANY

(Alberta Northeast Gas (Producers Mktg.))

AVG Daily Quantity

Total Price (Waddington. NY)

Commodity Component

Demand Component

Reservation Fee Component

NIAGARA MOHAWK POWER CORPORATION (TCGS Ltd.)

AVG Daily Quantity

Total Price (Waddington, NY)

Commodity Component

Demand Component

Reservation Fee Component
Auth.

Vols

25.2

$\begin{array}{lll}25.2 & 25.2 & 25.0 \\ 2.74 & 2.67 & 2.60 \\ 1.80 & 1.72 & 1.64 \\ 0.94 & 0.95 & 0.96\end{array}$

0.00

0.00

5.3

$\begin{array}{rrr}5.3 & 4.6 & 5.3 \\ \frac{2.74}{1.80} & \frac{2.67}{1.72} & \frac{2.60}{1.64} \\ 0.94 & 0.95 & 0.96 \\ 0.00 & 0.00 & 0.00\end{array}$

4.6

\begin{tabular}{rrr}
4.6 & 4.6 & 4.6 \\
2.82 & 2.76 & 2.69 \\
\hline 1.82 & 1.77 & 1.69 \\
1.00 & 0.99 & 1.00 \\
0.00 & 0.00 & 0.00
\end{tabular}

3.0

\begin{tabular}{rr}
3.0 & 3.0 \\
2.74 & 2.67 \\
\hline 1.80 & 1.7 \\
0.94 & 0.95 \\
0.00 & 0.00
\end{tabular}

51.0

0.00

\begin{tabular}{rr}
3.0 & 3.0 \\
67 & 2.58 \\
\hline
\end{tabular}

$\frac{2.58}{.72} \quad \frac{2.58}{1.62}$
1996

1996

1996

1996

$\begin{array}{lllll}1996 & 1996 & 1997 & 1997 & 1997\end{array}$

Dec. Jan

Eeb. Mar.

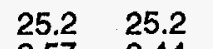

$\frac{2.57}{1.62}$

1.62
0.95

0.95
0.00

25.2
2.44
1.50
0.94

25.2
2.27
1.30
0.97

25.2
2.38
1.40
0.98
0.00

25.2
$\frac{3.28}{2.27}$
1.01

$\frac{25.2}{2.86}$

2.87

0.99
0.00

\begin{tabular}{l}
25.2 \\
3.89 \\
\hline 2.90 \\
0.99
\end{tabular}

0.99
0.00

\begin{tabular}{ll}
25.2 & 25.2 \\
3.37 & 2.62 \\
\hline 2.39 & 1.66 \\
0.98 & 0.96 \\
0.00 & 0.00
\end{tabular}

\begin{tabular}{lr}
.3 & 5.3 \\
60 & 2.57 \\
\hline .64 & 1.62 \\
06 & 0.95 \\
0.00 & 0.00
\end{tabular}

5.3
2.44
1.50
0.94
0.00

5.3
2.27
1.30
0.97
0.00

5.3
2.38
1.40
0.98
0.00

5.3
$\frac{3.28}{2.27}$
1.01

5.3
3.86
2.87
0.99

$\begin{array}{r}5.3 \\ 3.89 \\ \hline\end{array}$

$\frac{3.89}{2.90}$

2.90
0.99
0.00

$\begin{array}{r}5.3 \\ 3.37 \\ \hline\end{array}$

$\frac{3.37}{2.39}$

0.98

1.66
0.96
0.00

.00

.6
53
.54
99

415,800

\begin{tabular}{lr}
4.6 & 4.6 \\
2.65 & 2.52 \\
\hline 1.66 & 1.53 \\
0.99 & 0.99 \\
0.00 & 0.00
\end{tabular}

4.6
2.34
1.35
0.99

4.6
2.47
1.46

$1.46 \quad \frac{3.16}{2.14}$

1.01
0.00

1.02
0.00

4.6
$\frac{3.71}{2.71}$
1.00
0.00

4.6
4.00
2.99
1.01
0.00

$\begin{array}{r}4.6 \\ 3.25 \\ \hline 2.25\end{array}$

2.25
1.00

1.00

1.54

1.54
0.99
0.00

0.00

3.0
2.56
1.61

2.56
1.61
0.95

3.0
2.44

2.44
1.49
0.95

3.0
2.26
1.31
0.95

3.0
2.38
1.42
0.96

$\begin{array}{r}3.0 \\ 3.28 \\ \hline 2.30\end{array}$

$\frac{3.28}{2.30}$

$\begin{array}{r}3.0 \\ 3.86 \\ \hline 2.90\end{array}$

2.90
0.96

0.96
0.00

\begin{tabular}{r}
3.0 \\
3.89 \\
\hline 2.92 \\
0.97
\end{tabular}

$\begin{array}{r}3.0 \\ 3.37 \\ \hline\end{array}$

$\frac{3.37}{2.41}$
0.96

0.96
0.00

3.0
2.62
1.67
0.95

0.00

\begin{tabular}{lll}
51.0 & 51.0 & 51.0 \\
3.10 & 2.66 & 2.87 \\
\hline 2.17 & 1.74 & 1.91 \\
0.93 & 0.92 & 0.96 \\
0.00 & 0.00 & 0.00
\end{tabular}

\begin{tabular}{l}
51.0 \\
3.13 \\
\hline 2.22 \\
0.91 \\
0.00
\end{tabular}

\begin{tabular}{l}
51.0 \\
2.78 \\
\hline 1.87 \\
0.91 \\
0.00
\end{tabular}

51.0
2.25
1.29
0.96
0.00

50.9
2.26
1.32
0.94
0.00

\begin{tabular}{l}
51.0 \\
3.31 \\
\hline 2.30 \\
1.01 \\
0.00
\end{tabular}

\begin{tabular}{l}
50.9 \\
4.51 \\
\hline 3.56 \\
0.95 \\
0.00
\end{tabular}

51.0

4.65

3.69
0.96

0.96
0.00

\begin{tabular}{l}
51.0 \\
3.56 \\
\hline 2.52 \\
1.04 \\
0.00
\end{tabular}

51.0

2.20

1.27

0.93

$4,590,000$

$4,590,000$
415,800

YTD

$, 268,000$

472,500

268,560

268,560 


\section{VOLUME AND PRICE REPORT}

\section{Long-Term Imports}

Volumes (MMct/d) \& Prices (\$MMBTU) of Natural

Gas Imported During the Past 12 Months 04/01/96 - 03/31/97

Auth 1990

$\begin{array}{llllllllllll}1996 & 1996 & 1996 & 1996 & 1996 & 1996 & 1996 & 1996 & 1996 & 1997 & 1997 & 1997\end{array}$

Long-Term Importer

NORTH CANADIAN MARKETING CORPORATION (1)

(North Canadian Marketing Inc.)

AVG Daily Quantity

Total Price (Eastport, Idaho)

Commodity Component

Demand Component

Reservation Fee Component

NORTH JERSEY ENERGY ASSOCIATES

(ProGas Limited)

AVG Daily Quantity

Total Price (Niagara Falls, NY)

Demand Component

Reservation Fee Component
25.0

$\begin{array}{rrr}0.0 & 0.0 & \\ 0.00 & 0.00 & 0.0 \\ 0.00 & 0.00 & 0.00 \\ 0.00 & 0.00 & 0.00 \\ 0.00 & 0.00 & 0.00\end{array}$

24.0

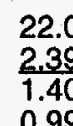

22.0
2.39
1.40
0.99
0.00

22.0
2.35
1.40
0.95
0.00

$\begin{array}{ll}22.0 & 22.1 \\ 2.35 & 2.41 \\ 1.40 & 1.42 \\ 0.95 & 0.99 \\ 0.00 & 0.00\end{array}$

48.0

$\begin{array}{llll}48.7 & 48.9 & 49.0 & 48.9 \\ \frac{3.13}{2.14} & \frac{3.18}{2.23} & \frac{3.21}{2.22} & \frac{3.18}{2.23} \\ 0.99 & 0.95 & 0.99 & 0.95\end{array}$

0.99

0.00

10.0

$\begin{array}{lll}10.0 & 10.0 & 10 . \\ \frac{2.51}{2.06} & \frac{2.00}{1.55} & \frac{2.0}{1.5} \\ 0.45 & 0.45 & 0.47 \\ 0.00 & 0.00 & 0.00\end{array}$

$0.00 \quad 2.84$

.00

0.00

0.00

0.00

0.00 $\begin{array}{lll}0 & 13.7 & 17.9\end{array}$

$\frac{2.60}{2.60}$

2.60
0.00

0.00
0.00

16.8
2.73
2.73
0.00
0.00

$\begin{array}{r}17.7 \\ 2.59 \\ \hline 1.82\end{array}$

$\frac{2.59}{1.82}$

0.77
0.00

18.3
2.46

$\frac{2.46}{1.92}$

0.54
0.00

14.4
2.69
1.92
0.77
0.00

13.1

$\frac{2.78}{1.92}$

0.86

$\frac{6.39}{1.92}$

$\frac{6.39}{1.92}$

$4.47 \quad 0.00$

0.00
0.00

NORTHEAST ENERGY ASSOCIATES

(ProGas Limited)

Total Price (Niagara Falls. NY)

Commodity Component

Demand Component
Reservation Fee Component

NORTHERN MINNESOTA UTILITIES

(TCGS Ltd.)

AVG Daily Quantity

Total Price (Noyes, Minnesota)

Commodity Component

Demand Component

Reservation Fee Component

NORTHERN MINNESOTA UTILITIES - EASTERN MARKET

(TCGS Ltd.)

AVG Daily Quantity

Total Price (International Falls, MN)

Commodity Component

Demand Component

Reservation Fee Component

29

$\begin{array}{lll}17.4 & 16.6 & 15.9 \\ \frac{1.66}{1.32} & \frac{1.48}{1.13} & \frac{1.41}{1.03} \\ 0.34 & 0.35 & 0.38 \\ 0.00 & 0.00 & 0.00\end{array}$

$\begin{array}{ll}22.0 & 22.0 \\ 2.38 & 2.38 \\ 1.43 & 1.43 \\ 0.95 & 0.95 \\ 0.00 & 0.00\end{array}$

22.1
2.40
1.42
0.98

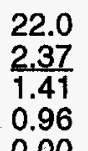

22.1
2.40
1.40

22.5

22.4
2.36
1.41

22.4

.46

22.4
$\frac{2.36}{1.43}$

0.00

0.93

0.05

$\begin{array}{ll}1.04 & 0.93 \\ 0.00 & 0.00\end{array}$

$2,011,849 \quad 2,011,849$

$2,011,849 \quad 2,011,849$

1097
IOTAL

482,882

482,882

00

$4,460,397$

$4,460,397$

$\begin{array}{llllllll}\frac{3.18}{2.23} & \frac{3.21}{2.23} & \frac{3.18}{2.22} & \frac{3.21}{2.21} & \frac{3.15}{2.22} & \frac{3.17}{2.22} & \frac{3.34}{2.30} & \frac{3.24}{2.31}\end{array}$

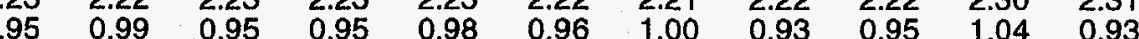

0.00

(1) This supply is sold to Hermiston cogeneration facility. 
VOLUME AND PRICE REPORT

\section{Long-Term Imports}

Volumes (MMcf/d) \& Prices (\$MMBTU) of Natural Gas Imported During the Past 12 Months 04/01/96 - 03/31/97
Long-Term Importer

Vols.

1996
Apr.

NORTHERN MINNESOTA UTILITIES - WESTERN MARKET

\section{(TCGS Lid.)}

AVG Daily Quantity

Total Price (Warroad, Minnesota)

Commodity Component

Comand Component

1.5

$\begin{array}{ll}\frac{1.3}{2.11} & 1 \\ 2.11 & 1 \\ 0.00 & 0.00 \\ 0.00 & 0.00\end{array}$

Reservation Fee Component

NORTHERN STATES POWER COMPANY

(Amoco Canada (WI))

AVG Daily Quantity

Total Price (Noyes. Minnesota)

Commodity Component

Demand Component

Reservation Fee Component

15.0

$\begin{array}{llll}14.8 & 14.8 & 14.8 & 14.7 \\ \frac{1.72}{1.27} & \frac{1.68}{1.27} & \frac{1.68}{1.27} & 1.68 \\ 0.33 & 0.29 & 0.29 & 0.29 \\ 0.12 & 0.12 & 0.12 & 0.12\end{array}$

$\begin{array}{rrr}2.6 & 4.9 & 4.9 \\ \frac{3.72}{2.41} & 2.66 & 2.74 \\ 0.94 & 0.90 & 1.96 \\ & 0.56 & 0.58\end{array}$

$\begin{array}{lll}0.94 & 0.56 & 0.58 \\ 0.37 & 0.20 & 0.20\end{array}$

(Canadian Occidental (WI))

AVG Daily Quantity

Total Price (Noyes. Minnesota)

Commodity Component

Demand Component

Reservation Fee Component

NORTHERN STATES POWER COMPANY

(ProGas Limited (WI))

AVG Daily Quantity

Total Price (Noves, Minnesota)

Commodity Component

Demand Component

Reservation Fee Component

NORTHERN STATES POWER COMPANY (MINNESOTA)

(Amoco Canada (MN)

AVG Daily Quantity

16.0

$\begin{array}{rrr}7.5 & 5.1 & 5 \\ \frac{2.46}{1.99} & \frac{2.57}{1.88} & 2.63 \\ 0.47 & 0.69 & 0.7 \\ 0.00 & 0.00 & 0.00\end{array}$

5.1
2.63
1.91
0.72
0.00

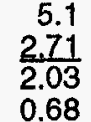

$\frac{2.71}{2.03}$

0.68
0.00

1996
Aug.

Total Price (Noyes, Minnesota)

Commodity Component

Demand Component

Reservation Fee Component

$\begin{array}{rrrrrrrrrrrr}15.3 & 14.6 & 10.2 & 6.4 & 9.8 & 11.7 & 13.0 & 15.8 & 15.9 & 15.8 & 15.9 & 15.6 \\ \frac{2.32}{1.80} & \frac{2.20}{1.67} & \frac{2.41}{1.70} & \frac{2.92}{1.83} & \frac{2.39}{1.76} & \frac{1.91}{1.29} & \frac{1.75}{1.20} & \frac{2.13}{1.62} & \frac{2.67}{2.18} & \frac{3.29}{2.79} & \frac{2.77}{2.23} & \frac{1.73}{1.23} \\ 0.52 & 0.53 & 0.71 & 1.09 & 0.63 & 0.62 & 0.55 & 0.51 & 0.49 & 0.50 & 0.54 & 0.50 \\ 0.00 & 0.00 & 0.00 & 0.00 & 0.00 & 0.00 & 0.00 & 0.00 & 0.00 & 0.00 & 0.00 & 0.00\end{array}$

1996

1097

TOTAL MCf

TOTAL Mcf

176,319

176,319

$\frac{1.18}{1.18}$

1.18

$0.00 \quad 0.00$

$0.00 \quad 0.00$

$\begin{array}{ll}0.00 & 0.00\end{array}$

0.00

$0.00 \quad 0.00$

.

4.9

$\begin{array}{lll}14.7 & 14.3 & 14.7\end{array}$

$\frac{1.67}{1.27} \cdot \frac{1.71}{1.27} \quad \frac{1.65}{1.27}$

14.9

14.9

14.8

14.8
2.41

$\frac{2.41}{1.93} \quad \frac{2.10}{1.66}$

1.27

1.27
0.25

1.93

$0.29 \quad 0.25$

0.12

0.13

0.12

0.12

0.12

0.19 .

$0.19 \quad 0.19$

$1,333,698$

$1,333,698$

674,865

674,999

674,999

$1,416,775$

$1,416,775$ 


\section{VOLUME AND PRICE REPORT}

\section{Long-Term Imports}

Volumes (MMct/d) \& Prices (\$MMBTU) of Natural

Gas Imported During the Past 12 Months 04/01/96 - 03/31/97

Long-Term importer

$\begin{array}{llllllllll}\text { Auth. } & 1996 & 1996 & 1996 & 1996 & 1996 & 1996 & 1996 & 1996 & 1996\end{array}$

Vols Apr May Jun Jul Aug Sep Oct Nov Dec 1997

19971997

1097

IOTAL MaI

YTD

NORTHERN STATES POWER COMPANY (MINNESOTA)

(TCGS Ltd.)

AVG Daily Quantity

19.4

Jotal Price (Noyes. Min

Commodity Component

Reservation Fee Component

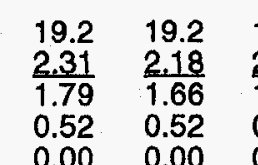

19.1
2.24
1.70
0.54
0.00

$\begin{array}{ll}16.4 & 18 . \\ 2.44 & 2.83 \\ 0.61 & 0.5\end{array}$

\begin{tabular}{ll}
18.2 & 18.2 \\
2.31 & 1.86 \\
\hline 1.77 & 1.29 \\
0.54 & 0.57 \\
0.00 & 0.00
\end{tabular}

18.6
2.88
2.28
0.60
0.00

19.9 0.00

NORTHERN UTILITIES, INC.

(Renaissance Energy Ltd.)

AVG Daily Quantity

Total Price (Niagara Falls. NY)

Commodity Component

Demand Component

Reservation Fee Component

$\begin{array}{lrr}1.0 & 1.0 & \\ \frac{3.30}{2.13} & \frac{3.39}{2.22} & \frac{3}{2} \\ 1.17 & 1.17 & 1 \\ 0.00 & 0.00 & 0\end{array}$

$\begin{array}{ll}1.0 & 1.0 \\ \frac{3.38}{2.21} & 3.20 \\ 1.17 & 1.17 \\ 0.00 & 0.00\end{array}$

$\begin{array}{rr}\frac{1.0}{3.20} \\ 2.03 \\ 1.17 & 1 \\ 0.00 & 1.17\end{array}$

\begin{tabular}{rrr}
1.0 & 1.0 & 1.0 \\
2.93 & 2.48 & 2.61 \\
\hline 1.76 & 1.31 & 1.44
\end{tabular}

$\frac{2.93}{1.76} \quad \frac{2.48}{1.31} \quad \frac{2.61}{1.44}$

NORTHWEST NATURAL GAS COMPANY

(Amoco Canada)

AVG Daily Quantity

Total Price (Sumas, Washington)

Commodity Component

Demand Component

Reservation Fee Component

\begin{tabular}{rr}
6.6 & 7.9 \\
\hline 1.27 & 1.15 \\
\hline 0.64 & 0.62 \\
0.63 & 0.53 \\
0.00 & 0.00
\end{tabular}

6.9
1.24
0.63
0.61
0.00

\begin{tabular}{r}
6.4 \\
1.43 \\
\hline 0.78 \\
0.65 \\
0.00
\end{tabular}

\begin{tabular}{r}
7.9 \\
1.19 \\
\hline 0.66 \\
0.53 \\
0.00
\end{tabular}
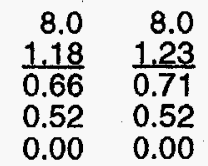

\begin{tabular}{r}
7.7 \\
1.85 \\
\hline 1.24 \\
0.61 \\
0.00
\end{tabular}

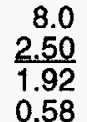

6.6
2.64
1.88
0.76
0.00

\begin{tabular}{r}
6.9 \\
1.94 \\
\hline 1.13 \\
0.81 \\
0.00
\end{tabular}

\begin{tabular}{r}
8.0 \\
1.20 \\
\hline 0.62 \\
0.58 \\
0.00
\end{tabular}

NORTHWEST NATURAL GAS COMPANY

(CanWest Gas Supply)

AVG Daily Quantity

Total Price (Sumas, Washington)

Commodity Component

Demand Component

Reservation Fee Component

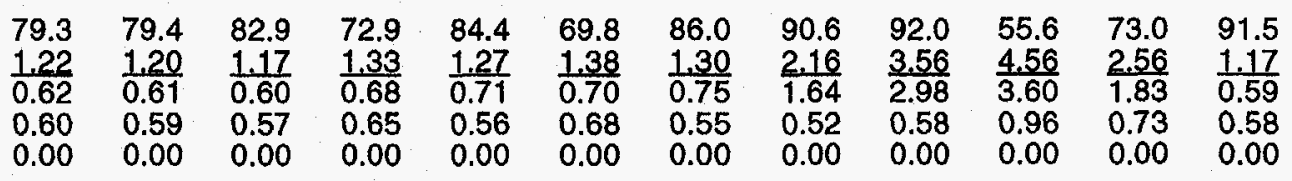

\section{NORTHWEST NATURAL GAS COMPANY}

(Poco Petroleums Limited)

AVG Daily Quantity

Total Price (Eastport, Idaho)

Commodity Componen

Demand Component

Reservation Fee Component

\begin{tabular}{|c|c|c|c|c|c|c|c|c|c|c|c|}
\hline $\begin{array}{l}0.6 \\
.21 \\
60\end{array}$ & $\begin{array}{l}10.6 \\
1.19 \\
0.60 \\
0.34 \\
0.25\end{array}$ & $\begin{array}{l}10 . \\
1.1 \\
0.5 \\
0.3 \\
0.2\end{array}$ & $\begin{array}{l}1.2 \\
0.6 \\
0.3 \\
0.2\end{array}$ & $\begin{array}{l}10 \\
1.2 \\
0\end{array}$ & $\begin{array}{l}10.2 \\
1.27 \\
0.64\end{array}$ & $\begin{array}{l}14.8 \\
1.21 \\
0.69 \\
0.27 \\
0.25\end{array}$ & $\begin{array}{l}14.9 \\
1.77 \\
1.24\end{array}$ & & $\begin{array}{r}7.2 \\
5.26 \\
4.17 \\
0.57 \\
0.52\end{array}$ & & $\begin{array}{l}9 \\
\frac{3}{1} \\
\end{array}$ \\
\hline
\end{tabular}




\section{VOLUME AND PRICE REPORT}

\section{Long-Term Imports}

Volumes (MMct/d) \& Prices (\$MMBTU) of Natural

Gas Imported During the Past 12 Months 04/01/96 - 03/31/97

\section{Long-Term Imperter}

NORTHWEST NATURAL GAS COMPANY

(Summit Resources Limited)

AVG Daily Quantity

Commodity Component

Demand Component

Reservation Fee Component

NORTHWEST NATURAL GAS COMPANY

(Westcoast Gas Services)

AVG Daily Quantity

Total Price (Eastport, Idaho)

Commodity Component

Demand Component

Reservation Fee Component

NUI CORPORATION (1)

(TCGS Ltd.)

Commodity Component

Demand Component

Reservation Fee Component

NW ALASKAN PIPELINE CO-Pacific Interstate

(Pan-Alberta Gas Ltd.)

AVG Daily Quantity

Demand Component

NW ALASKAN PIPELINE CO-Pan-Alberta Gas (1) (2)

(Pan-Alberta Gas Ltd.)

AVG Daily Quantity

Total Price (Port of Morgan, MT)

Commodity Component

Demand Component

Reservation Fee Component

8.0

23.3

240.0
Total Price (Eastport. Idaho)

AVG Daily Quantity

Total Price (Niadara Falls. NY)

Iotal Price (Eastport. Idaho)

Commodity Component

Reservation Fee Component $\begin{array}{lllllllllllll}\text { Auth. } & 1996 & 1996 & 1996 & 1996 & 1996 & 1996 & 1996 & 1996 & 1996 & 1997 & 1997 & 1997\end{array}$

Vols. Apr. May. Jun. Jul Aug. Sep. Oct Nov Dec. Jan. Feb. Mar.

\begin{tabular}{rr}
4.9 & 4.9 \\
1.33 & 1.31 \\
\hline 0.91 & 0.91 \\
0.42 & 0.40
\end{tabular}

$0.42 \quad 0.40$

$0.00 \quad 0.00$

$\begin{array}{lll}13.7 & 13.5 & 13 \\ \frac{1.36}{0.68} & \frac{1.35}{0.66} & 1.2 \\ 0.42 & 0.43 & 0.43 \\ 0.26 & 0.26 & 0.26\end{array}$

2.0

$\begin{array}{rrr}2.0 & 1.9 & 3.29 \\ \frac{3.2 .80}{2.53} & \frac{2.05}{2.05} & 2.23 \\ 0.76 & 0.75 & 0.78 \\ 0.00 & 0.00 & 0.00\end{array}$

$240.0 \quad 240.0$

$0.72 \quad 0.72$

$\begin{array}{lll}0.43 & 0.44 & \frac{0.78}{0.49}\end{array}$

$\begin{array}{lll}0.29 & 0.28 & 0.29 \\ 0.00 & 0.00 & 0.00\end{array}$

\begin{tabular}{rr}
4.9 & 4.8 \\
1.33 & 1.28 \\
\hline 0.91 & 0.91 \\
0.42 & 0.37
\end{tabular}

0.00

0.00

4.8

$\frac{1.28}{0.91}$

0.37

0.00

$\begin{array}{r}4.8 \\ 1.30 \\ \hline 0.91\end{array}$

$\frac{1.30}{0.91}$

0.39
0.00

7.4

$\frac{1.42}{1.17}$

0.25

0.25
0.00

$\begin{array}{r}7.6 \\ 1.42 \\ \hline 1.17\end{array}$

1.17
0.25

0.25
0.00

\begin{tabular}{r}
7.6 \\
1.41 \\
\hline 1.17 \\
0.24
\end{tabular}

$\begin{array}{r}7.6 \\ 1.42 \\ \hline 1.17\end{array}$

1.17

0.25
0.00

$\begin{array}{r}7.6 \\ 1.18 \\ \hline\end{array}$

$\frac{1.18}{0.95}$

0.23

7.5
0.94
0.69

0.25
0.00

$0.00 \quad 0.00$

200.0

\begin{tabular}{|c|c|c|c|c|c|c|c|c|c|c|c|}
\hline $\begin{array}{l}99.9 \\
2.24 \\
1.94 \\
0.30\end{array}$ & $\begin{array}{r}199.2 \\
2.09 \\
1.80 \\
0.29 \\
0.00\end{array}$ & $\begin{array}{r}199.8 \\
2.10 \\
1.80 \\
0.30 \\
0.00\end{array}$ & $\begin{array}{r}199 . \\
1.9 \\
1.7 \\
0.2 \\
0.0\end{array}$ & $\begin{array}{r}199.9 \\
2.28 \\
2.08 \\
0.20 \\
0.00\end{array}$ & $\begin{array}{r}198.9 \\
1.72 \\
1.51 \\
0.21 \\
0.00\end{array}$ & $\begin{array}{c}199 \\
1.8 \\
1.5 \\
0.2 \\
0.8\end{array}$ & $\begin{array}{r}19 \\
2\end{array}$ & & $\begin{array}{l}3.44 \\
0.25 \\
0.00\end{array}$ & & \\
\hline
\end{tabular}

$\begin{array}{rrrr}1.8 & 13.0 & 9.9 & 19.4 \\ \frac{1.17}{0.62} & \frac{1.19}{0.65} & \frac{1.54}{0.82} & \frac{1.32}{0.83}\end{array}$

20.5

22.3
1.45

20.9

$19.5 \quad 22.2$

$\frac{1.48}{0.96} \quad \frac{1.41}{0.98}$

0.28

0.27
0.27

0.35

0.18

0.30
0.17

0.16

0.17

0.18

0.16

$\begin{array}{rrrrrrrrr}1.3 & 1.3 & 0.7 & 1.9 & 2.0 & 1.7 & 1.5 & 1.3 & 1.9 \\ \frac{3.64}{2.52} & \frac{3.28}{2.17} & \frac{3.79}{1.67} & \frac{2.46}{1.70} & \frac{3.34}{2.58} & \frac{4.55}{3.68} & \frac{4.74}{3.79} & \frac{3.98}{2.74} & \frac{2.38}{1.61} \\ 1.12 & 1.11 & 2.12 & 0.76 & 0.76 & 0.87 & 0.95 & 1.24 & 0.77 \\ 0.00 & 0.00 & 0.00 & 0.00 & 0.00 & 0.00 & 0.00 & 0.00 & 0.00\end{array}$

0.00

.00

0000

237.3
0.95
0.74
0.21
0.00

$\begin{array}{lr}37.3 & 239.3 \\ \frac{0.95}{0.74} & 1.4 \\ 0.21 & 0.2 \\ 0.00 & 0.0\end{array}$

$\begin{array}{ll}9.3 & 237.2 \\ .44 & 0.9 \\ .23 & 0.77 \\ .21 & 0.22 \\ .00 & 0.00\end{array}$

\begin{tabular}{lr}
37.2 & 238. \\
0.99 & 1.04 \\
\hline 0.77 & 0.83 \\
0.22 & 0.21 \\
0.00 & 0.00
\end{tabular}

\begin{tabular}{lr}
8.1 & 238.7 \\
04 & 1.87 \\
\hline 83 & 1.66 \\
21 & 0.21 \\
00 & 0.00
\end{tabular}

$\begin{array}{llll}240.0 & 222.5 & 194.9 & 239.7\end{array}$ $\begin{array}{llll}\frac{3.00}{2.79} & \frac{3.55}{3.25} & \frac{1.96}{1.59} & \frac{0.90}{0.62}\end{array}$ $\begin{array}{llll}2.79 & 3.25 & 1.59 & 0.62 \\ 0.21 & 0.30 & 0.37 & 0.28\end{array}$

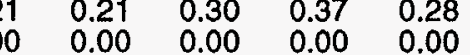

$1 \mathrm{Q} 97$

TOTAL Mct

682,827

682,827

$1,881,674$

$1,881,674$

140,184

140,184

(1) Gas supplies NUl's Waverly Gas Service and Valley Clties Gas Service LDC divisions.

(2) Effective November 1,1993, the Northern Natural contract was assumed by Pan-Alberta Gas (U.S.) Inc. 


\section{VOLUME AND PRICE REPORT}

\section{Long-Term Imports}

Volumes (MMcfld) \& Prices (\$MMBTU) of Natural

Gas Imported During the Past 12 Months 04/01/96 - 03/31/97

Leng-Term Importer

Auth.
Vols.

$1996 \quad 1996 \quad 1996$

Apr.

Mav.

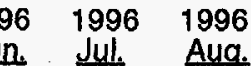

Aug.

1996
Sep.

1996

1996

1996

NW ALASKAN PIPELINE CO-Pan-Alberta Gas (II)

(Pan-Alberta Gas Ltd.)

AVG Daily Quantity

Total Price (Port of Morgan. MT)

Commodity Component

Demand Component

Reservation Fee Component

600.0

$\begin{array}{rrrr}601.4 & 599.8 & 598.4 & 598.0 \\ 1.86 & 1.62 & 1.63 & 1.73 \\ 1.85 & 1.61 & 1.62 & 1.73 \\ 0.01 & 0.01 & 0.01 & 0.00 \\ 0.00 & 0.00 & 0.00 & 0.00\end{array}$

599.7
$\frac{1.63}{1.63}$
0.00
0.00

596.8
$\frac{1.32}{1.32}$
0.00
0.00

599.4
1.49
1.49
0.00
0.00

597.1
$\frac{2.17}{2.17}$
0.00
0.00

$\begin{array}{r}597.8 \\ 3.04 \\ \hline\end{array}$

$\begin{array}{llll}0.00 & 0.00 & 0.00 & 0.00\end{array}$

0.00

50.0

\begin{tabular}{|c|c|c|c|c|c|c|c|c|c|c|c|}
\hline $\begin{array}{l}50.0 \\
2.06 \\
1.12 \\
0.94 \\
0.00\end{array}$ & $\begin{array}{l}50.0 \\
2.07 \\
1.10 \\
0.97 \\
0.00\end{array}$ & $\begin{array}{l}50.0 \\
2.08 \\
1.10 \\
0.98 \\
0.00\end{array}$ & $\begin{array}{l}50.0 \\
2.07 \\
1.10 \\
0.97 \\
0.00\end{array}$ & $\begin{array}{l}50.0 \\
2.09 \\
1.11 \\
0.98 \\
0.00\end{array}$ & $\begin{array}{l}50.0 \\
2.10 \\
1.13 \\
0.97 \\
0.00\end{array}$ & $\begin{array}{l}50.0 \\
2.14 \\
1.17 \\
0.97 \\
0.00\end{array}$ & $\begin{array}{l}50.0 \\
2.15 \\
1.17 \\
0.98 \\
0.00\end{array}$ & $\begin{array}{l}50.0 \\
2.19 \\
1.20 \\
0.99 \\
0.00\end{array}$ & $\begin{array}{l}50.0 \\
2.23 \\
1.24 \\
0.99 \\
0.00\end{array}$ & $\begin{array}{l}50.0 \\
2.28 \\
1.30 \\
0.98 \\
0.00\end{array}$ & $\begin{array}{l}50.0 \\
2.32 \\
1.34 \\
0.98 \\
0.00\end{array}$ \\
\hline
\end{tabular}

25.0

$\begin{array}{ll}25.0 & 25 \\ 2.06 & 2 \\ 1.12 & 1 \\ 0.94 & 0 \\ 0.00 & 0\end{array}$

$\begin{array}{ll}25.0 & 25.0 \\ 2.07 & 2.0 \\ 1.10 & 1.10 \\ 0.97 & 0.98 \\ 0.00 & 0.00\end{array}$

$\begin{array}{ll}25.0 & 25.0 \\ 2.08 & 2.07 \\ 1.10 & 1.10 \\ 0.98 & 0.97 \\ 0.00 & 0.00\end{array}$

$25.0 \quad 25.0$

$25.0 \quad 25.0$

$\frac{2.07}{1.10} \quad \frac{2.09}{1.11}$

$0.97 \quad 0.98$

0.98
0.00

$\frac{2.10}{1.13}$

1.13
0.97
0.00

25.0
2.14
1.17

$\frac{2.14}{1.17}$

0.97
0.00

25.0
2.15
1.17

$\frac{2.15}{1.17}$

0.98
0.00

25.0
$\frac{2.19}{1.20}$
0.99
0.00

25.0
2.23
1.2
0.99

.24

$25.0 \quad 25.0$

.28

Reservation Fee Component

OCEAN STATE POWER ॥

(TCGS Ltd.)

AVG Daily Quantity

Total Price (Niagara Falls. NY)

25.0

$\begin{array}{llllllllllll}25.0 & 25.0 & 25.0 & 25.0 & 25.0 & 25.0 & 25.0 & 25.0 & 25.0 & 25.0 & 25.0 & 25.0 \\ \frac{2.06}{1.16} & \frac{2.07}{1.14} & \frac{2.07}{1.16} & \frac{2.07}{1.14} & \frac{2.08}{1.14} & \frac{2.11}{1.18} & \frac{2.14}{1.20} & \frac{2.16}{1.20} & \frac{2.19}{1.20} & \frac{2.23}{1.25} & \frac{2.28}{1.32} & \frac{2.32}{1.36} \\ 0.90 & 0.93 & 0.91 & 0.93 & 0.94 & 0.93 & 0.94 & 0.96 & 0.99 & 0.98 & 0.96 & 0.96 \\ 0.00 & 0.00 & 0.00 & 0.00 & 0.00 & 0.00 & 0.00 & 0.00 & 0.00 & 0.00 & 0.00 & 0.00\end{array}$

Demand Component

Reservation Fee Component

ORANGE AND ROCKLAND UTILITIES, INC.

(Wascana Energy Marketing)

AVG Daily Quantity

Total Price (Niagara Falls. NY)

Commodity Component

Demand Component

Reservation Fee Component

$\begin{array}{llllllllllll}24.8 & 24.9 & 25.0 & 24.9 & 24.9 & 25.0 & 24.9 & 25.0 & 25.0 & 24.9 & 24.8 & 24.8 \\ \frac{3.38}{2.22} & \frac{2.95}{1.87} & \frac{3.16}{2.01} & \frac{3.41}{2.26} & \frac{3.07}{1.96} & \frac{2.60}{1.51} & \frac{2.62}{1.54} & \frac{3.50}{2.30} & \frac{4.55}{3.29} & \frac{4.66}{3.38} & \frac{3.75}{2.47} & \frac{2.53}{1.46} \\ 0.91 & 0.87 & 0.92 & 0.89 & 0.89 & 0.92 & 0.90 & 0.94 & 0.89 & 0.90 & 1.00 & 0.90 \\ 0.25 & 0.21 & 0.23 & 0.26 & 0.22 & 0.17 & 0.18 & 0.26 & 0.37 & 0.38 & 0.28 & 0.17\end{array}$

1097

IOTAL MCI

$4,288,725$

$54,288,725$

$4,500,000$

$4,500,000$

$2,250,000$

$2,250,000$

$2,250,000$

$2,250,000$

$2,237,636$

$2,237,636$ 


\section{VOLUME AND PRICE REPORT}

Long-Term Imports

Volumes (MMct/d) \& Prices ( $\$ M M B T U$ ) of Natural

Gas Imported During the Past 12 Months 04/01/96 - 03/31/97

\section{Long-Term Importer}

\section{ORCHARD GAS CORPORATION (1)}

(ProGas Limited

AVG Daily Quantity

Total Price (Waddington. NY)

Commodity Component

Demand Component

Reservation Fee Component

PAN ENERGY LNG SALES, INC. (2)

(Sonatrading)

AVG Daily Quantity

Total Price (Lake Charles. LA)

Commodity Component

Demand Component

Reservation Fee Component

PAWTUCKET POWER ASSOCIATES (3)

(Anderson Exploration Ltd.)

AVG Daily Quantity

Total Price (Waddington. NY)

Commodity Component

Demand Component

Reservation Fee Component

PAWTUCKET POWER ASSOCIATES

(Tarragon Oil \& Gas Limited)

AVG Daily Quantity

Total Price (Waddington. NY)

Commodity Component

Demand Component

Reservation Fee Component

PEOPLES NATURAL GAS COMPANY

(TCGS Lid.)

AVG Daily Quantity

Total Price (Noyes, Minnesota)

Commodity Component

Demand Component

Reservation Fee Component

\begin{tabular}{|c|c|c|c|c|c|c|c|c|c|c|c|c|}
\hline $\begin{array}{l}\text { Auth. } \\
\text { Vols. }\end{array}$ & $\begin{array}{l}1996 \\
\text { Apr. }\end{array}$ & $\begin{array}{l}1996 \\
\text { May. }\end{array}$ & $\begin{array}{r}1996 \\
\text { Jun. }\end{array}$ & $\begin{array}{l}1996 \\
\text { Jul. }\end{array}$ & $\begin{array}{l}1996 \\
\text { Aug. }\end{array}$ & $\begin{array}{l}1996 \\
\text { Sep. }\end{array}$ & $\begin{array}{l}1996 \\
\text { Oct. }\end{array}$ & $\begin{array}{l}1996 \\
\text { Nov. }\end{array}$ & $\begin{array}{l}1996 \\
\text { Dec. }\end{array}$ & $\begin{array}{l}1997 \\
\text { Jan. }\end{array}$ & $\begin{array}{l}1997 \\
\text { Feb. }\end{array}$ & $\begin{array}{l}1997 \\
\text { Mar. }\end{array}$ \\
\hline 25.0 & $\begin{array}{l}25.0 \\
3.17 \\
2.21 \\
0.96 \\
0.00\end{array}$ & $\begin{array}{l}25.0 \\
3.21 \\
2.22 \\
0.99 \\
0.00\end{array}$ & $\begin{array}{l}25.0 \\
3.18 \\
2.18 \\
1.00 \\
0.00\end{array}$ & $\begin{array}{l}25.0 \\
3.06 \\
2.07 \\
0.99 \\
0.00\end{array}$ & $\begin{array}{l}24.8 \\
3.07 \\
2.07 \\
1.00 \\
0.00\end{array}$ & $\begin{array}{l}21.3 \\
3.08 \\
2.09 \\
0.99 \\
0.00\end{array}$ & $\begin{array}{l}25.0 \\
3.19 \\
2.18 \\
1.01 \\
0.00\end{array}$ & $\begin{array}{l}25.0 \\
3.17 \\
2.16 \\
1.01 \\
0.00\end{array}$ & $\begin{array}{l}25.0 \\
3.19 \\
2.19 \\
1.00 \\
0.00\end{array}$ & $\begin{array}{l}25.0 \\
3.26 \\
2.26 \\
1.00 \\
0.00\end{array}$ & $\begin{array}{l}22.8 \\
3.41 \\
2.42 \\
0.99 \\
0.00\end{array}$ & $\begin{array}{l}18.8 \\
3.54 \\
2.54 \\
1.00 \\
0.00\end{array}$ \\
\hline 123.3 & $\begin{array}{r}0.0 \\
0.00 \\
0.00 \\
0.00 \\
0.00\end{array}$ & $\begin{array}{r}0.0 \\
0.00 \\
0.00 \\
0.00 \\
0.00\end{array}$ & $\begin{array}{r}0.0 \\
0.00 \\
0.00 \\
0.00 \\
0.00\end{array}$ & $\begin{array}{r}0.0 \\
0.00 \\
0.00 \\
0.00 \\
0.00\end{array}$ & $\begin{array}{r}0.0 \\
0.00 \\
0.00 \\
0.00 \\
0.00\end{array}$ & $\begin{array}{r}0.0 \\
0.00 \\
0.00 \\
0.00 \\
0.00\end{array}$ & $\begin{array}{r}0.0 \\
0.00 \\
0.00 \\
0.00 \\
0.00\end{array}$ & $\begin{array}{l}71.1 \\
2.00 \\
2.00 \\
0.00 \\
0.00\end{array}$ & $\begin{array}{l}74.0 \\
2.00 \\
2.00 \\
0.00 \\
0.00\end{array}$ & $\begin{array}{l}74.2 \\
2.00 \\
2.00 \\
0.00 \\
0.00\end{array}$ & $\begin{array}{l}74.2 \\
2.00 \\
2.00 \\
0.00 \\
0.00\end{array}$ & $\begin{array}{r}0.0 \\
0.00 \\
0.00 \\
0.00 \\
0.00\end{array}$ \\
\hline 8.2 & $\begin{array}{r}4.8 \\
3.40 \\
1.99 \\
1.41 \\
0.00\end{array}$ & $\begin{array}{r}4.6 \\
3.49 \\
1.99 \\
1.50 \\
0.00\end{array}$ & $\begin{array}{r}5.2 \\
\frac{3.43}{2.03} \\
1.40 \\
0.00\end{array}$ & $\begin{array}{r}4.6 \\
3.65 \\
2.16 \\
1.49 \\
0.00\end{array}$ & $\begin{array}{r}7.3 \\
2.94 \\
2.02 \\
0.92 \\
0.00\end{array}$ & $\begin{array}{r}7.1 \\
2.81 \\
1.82 \\
0.99 \\
0.00\end{array}$ & $\begin{array}{r}7.2 \\
2.60 \\
1.93 \\
0.67 \\
0.00\end{array}$ & $\begin{array}{r}7.6 \\
3.00 \\
2.03 \\
0.97 \\
0.00\end{array}$ & $\begin{array}{r}7.1 \\
\frac{3.48}{2.51} \\
0.97 \\
0.00\end{array}$ & $\begin{array}{r}7.4 \\
3.43 \\
2.48 \\
0.95 \\
0.00\end{array}$ & $\begin{array}{r}7.1 \\
2.63 \\
1.70 \\
0.93 \\
0.00\end{array}$ & $\begin{array}{r}5.7 \\
2.71 \\
1.51 \\
1.20 \\
0.00\end{array}$ \\
\hline 6.0 & $\begin{array}{r}3.4 \\
3.31 \\
1.90 \\
1.41 \\
0.00\end{array}$ & $\begin{array}{r}3.2 \\
3.48 \\
1.98 \\
1.50 \\
0.00\end{array}$ & $\begin{array}{r}3.4 \\
3.39 \\
1.99 \\
1.40 \\
0.00\end{array}$ & $\begin{array}{r}3.2 \\
3.52 \\
2.03 \\
1.49 \\
0.00\end{array}$ & $\begin{array}{r}5.2 \\
2.88 \\
1.96 \\
0.92 \\
0.00\end{array}$ & $\begin{array}{r}5.0 \\
2.93 \\
1.94 \\
0.99 \\
0.00\end{array}$ & $\begin{array}{r}5.1 \\
2.84 \\
2.17 \\
0.67 \\
0.00\end{array}$ & $\begin{array}{r}5.1 \\
3.37 \\
2.40 \\
0.97 \\
0.00\end{array}$ & $\begin{array}{r}5.0 \\
3.47 \\
2.50 \\
0.97 \\
0.00\end{array}$ & $\begin{array}{r}5.1 \\
3.35 \\
2.40 \\
0.95 \\
0.00\end{array}$ & $\begin{array}{r}5.0 \\
2.70 \\
1.77 \\
0.93 \\
0.00\end{array}$ & $\begin{array}{r}4.0 \\
2.95 \\
1.75 \\
1.20 \\
0.00\end{array}$ \\
\hline 9.0 & $\begin{array}{r}0.0 \\
0.00 \\
0.00 \\
0.00 \\
0.00\end{array}$ & $\begin{array}{r}0.0 \\
0.00 \\
0.00 \\
0.00 \\
0.00\end{array}$ & $\begin{array}{r}0.0 \\
0.00 \\
0.00 \\
0.00 \\
0.00\end{array}$ & $\begin{array}{r}0.0 \\
0.00 \\
0.00 \\
0.00 \\
0.00\end{array}$ & $\begin{array}{r}0.0 \\
0.00 \\
0.00 \\
0.00 \\
0.00\end{array}$ & $\begin{array}{r}0.0 \\
0.00 \\
0.00 \\
0.00 \\
0.00\end{array}$ & $\begin{array}{r}0.0 \\
0.00 \\
0.00 \\
0.00 \\
0.00\end{array}$ & $\begin{array}{r}0.0 \\
0.00 \\
0.00 \\
0.00 \\
0.00\end{array}$ & $\begin{array}{r}0.0 \\
0.00 \\
0.00 \\
0.00 \\
0.00\end{array}$ & $\begin{array}{r}0.0 \\
0.00 \\
0.00 \\
0.00 \\
0.00\end{array}$ & $\begin{array}{r}0.0 \\
0.00 \\
0.00 \\
0.00 \\
0.00\end{array}$ & $\begin{array}{r}0.0 \\
0.00 \\
0.00 \\
0.00 \\
0.00\end{array}$ \\
\hline
\end{tabular}

1Q97

TOTAL MCf

$1,993,750$

$1,993,750$

$4,378,479$

$4,378,479$

601,571

601,571

424,516

424,516

YTD

TOTAL MC

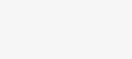

.
4

(1) Acts as agent for MASSPOWER and Granile State Gas Transmission System.

(3) Eailgate Price. 


\section{VOLUME AND PRICE REPORT}

\section{Long-Term Imports}

Volumes (MMct/d) \& Prices (\$MMBTU) of Natural

Gas Imported During the Past 12 Months 04/01/96 - 03/31/97

Long-Term Importer

PG ENERGY (1)

(TransCanada PipeLines Limited)

AVG Daily Quantity

Total Price (Niagara Falls, NY)

Commodity Component

Demand Component

Reservation Fee Component

PITTSFIELD GENERATING CO., L.P. (2)

(Anderson Exploration Ltd.)

AVG Daily Quantity

Total Price (Niagara Falls, NY)

Commodity Componen

Demand Component

Reservation Fee Component

PITTSFIELD GENEAATING CO., L.P. (3)

(Talisman Energy Inc.)

AVG Daily Quantity

Total Price (Niagara Falls. NY)

Commodity Component

Demand Component

Reservation Fee Component

POCO PETROLEUM INC.

(IGI Resources, Inc.)

AVG Daily Quantity

Total Price (Sumas, Washington)

Commodity Component

Demand Component

Reservation Fee Component

PORTAL MUNICIPAL GAS (4)

(SaskEnergy Inc.)

AVG Daily Quantity

Total Price (Portal. North Dakota)

Commodity Component

Demand Component

Reservation Fee Component

\begin{tabular}{|c|c|c|c|c|c|c|c|c|c|c|c|c|}
\hline $\begin{array}{l}\text { Auth. } \\
\text { Vols. }\end{array}$ & $\begin{array}{l}1996 \\
\text { Apr. }\end{array}$ & $\begin{array}{l}1996 \\
\text { May. }\end{array}$ & $\begin{array}{l}1996 \\
\text { Jun. }\end{array}$ & $\begin{array}{l}1996 \\
\text { Jul. }\end{array}$ & $\begin{array}{r}1996 \\
\text { Aug. }\end{array}$ & $\begin{array}{l}1996 \\
\text { Sep. }\end{array}$ & $\begin{array}{c}1996 \\
\text { Oct. }\end{array}$ & $\begin{array}{l}1996 \\
\text { Nov. }\end{array}$ & $\begin{array}{l}1996 \\
\text { Dec. }\end{array}$ & $\begin{array}{l}1997 \\
\text { Jan. }\end{array}$ & $\begin{array}{l}1997 \\
\text { Feb. }\end{array}$ & $\begin{array}{l}1997 \\
\text { Mar. }\end{array}$ \\
\hline 14.7 & $\begin{array}{l}14.9 \\
3.27 \\
2.53 \\
0.74 \\
0.00\end{array}$ & $\begin{array}{l}14.9 \\
2.77 \\
2.05 \\
0.72 \\
0.00\end{array}$ & $\begin{array}{l}15.0 \\
2.97 \\
2.23 \\
0.74 \\
0.00\end{array}$ & $\begin{array}{l}14.9 \\
3.24 \\
2.52 \\
0.72 \\
0.00\end{array}$ & $\begin{array}{l}14.9 \\
2.89 \\
2.17 \\
0.72 \\
0.00\end{array}$ & $\begin{array}{l}15.0 \\
2.41 \\
1.67 \\
0.74 \\
0.00\end{array}$ & $\begin{array}{l}14.9 \\
2.42 \\
1.70 \\
0.72 \\
0.00\end{array}$ & $\begin{array}{l}15.0 \\
\frac{3.34}{2.58} \\
0.76 \\
0.00\end{array}$ & $\begin{array}{l}15.0 \\
4.41 \\
3.68 \\
0.73 \\
0.00\end{array}$ & $\begin{array}{l}14.9 \\
4.51 \\
3.79 \\
0.72 \\
0.00\end{array}$ & $\begin{array}{l}14.9 \\
3.54 \\
2.74 \\
0.80 \\
0.00\end{array}$ & $\begin{array}{l}14 . \\
2.3 \\
1.6 \\
0.7 \\
0.0\end{array}$ \\
\hline 11.8 & $\begin{array}{r}9.0 \\
2.58 \\
1.74 \\
0.84 \\
0.00\end{array}$ & $\begin{array}{l}10.3 \\
2.56 \\
1.67 \\
0.89 \\
0.00\end{array}$ & $\begin{array}{r}8.4 \\
2.53 \\
1.64 \\
0.89 \\
0.00\end{array}$ & $\begin{array}{l}11.4 \\
2.60 \\
1.71 \\
0.89 \\
0.00\end{array}$ & $\begin{array}{l}11.3 \\
2.63 \\
1.75 \\
0.88 \\
0.00\end{array}$ & $\begin{array}{l}11.3 \\
2.69 \\
1.80 \\
0.89 \\
0.00\end{array}$ & $\begin{array}{l}11.3 \\
2.89 \\
1.99 \\
0.90 \\
0.00\end{array}$ & $\begin{array}{l}11.3 \\
2.92 \\
2.01 \\
0.91 \\
0.00\end{array}$ & $\begin{array}{l}11.8 \\
2.87 \\
1.98 \\
0.89 \\
0.00\end{array}$ & $\begin{array}{l}11.8 \\
2.80 \\
1.90 \\
0.90 \\
0.00\end{array}$ & $\begin{array}{l}11.4 \\
2.74 \\
1.85 \\
0.89 \\
0.00\end{array}$ & $\begin{array}{l}0.8 \\
0.0\end{array}$ \\
\hline 22.4 & $\begin{array}{l}17.2 \\
3.02 \\
2.13 \\
0.89 \\
0.00\end{array}$ & $\begin{array}{l}19.6 \\
2.80 \\
1.91 \\
0.89 \\
0.00\end{array}$ & $\begin{array}{l}15.9 \\
2.80 \\
1.91 \\
0.89 \\
0.00\end{array}$ & $\begin{array}{l}21.7 \\
2.94 \\
2.05 \\
0.89 \\
0.00\end{array}$ & $\begin{array}{l}21.5 \\
2.80 \\
1.92 \\
0.88 \\
0.00\end{array}$ & $\begin{array}{l}21.4 \\
2.69 \\
1.80 \\
0.89 \\
0.00\end{array}$ & $\begin{array}{l}21.5 \\
2.88 \\
1.98 \\
0.90 \\
0.00\end{array}$ & $\begin{array}{l}21.5 \\
3.15 \\
2.24 \\
0.91 \\
0.00\end{array}$ & $\begin{array}{l}22.4 \\
3.54 \\
2.65 \\
0.89 \\
0.00\end{array}$ & $\begin{array}{l}22.4 \\
3.39 \\
2.49 \\
0.90 \\
0.00\end{array}$ & $\begin{array}{l}21.8 \\
2.96 \\
2.07 \\
0.89 \\
0.00\end{array}$ & 0.0 \\
\hline 20.0 & $\begin{array}{l}19.1 \\
1.03 \\
0.38 \\
0.50 \\
0.15\end{array}$ & $\begin{array}{l}19.6 \\
0.98 \\
0.33 \\
0.50 \\
0.15\end{array}$ & $\begin{array}{l}18.8 \\
0.98 \\
0.33 \\
0.50 \\
0.15\end{array}$ & $\begin{array}{l}19.5 \\
1.01 \\
0.36 \\
0.50 \\
0.15\end{array}$ & $\begin{array}{l}19.7 \\
1.04 \\
0.39 \\
0.50 \\
0.15\end{array}$ & $\begin{array}{l}19.6 \\
1.06 \\
0.41 \\
0.50 \\
0.15\end{array}$ & $\begin{array}{l}19.6 \\
1.15 \\
0.50 \\
0.50 \\
0.15\end{array}$ & $\begin{array}{l}19.4 \\
2.22 \\
1.55 \\
0.52 \\
0.15\end{array}$ & $\begin{array}{l}19.5 \\
3.60 \\
2.95 \\
0.50 \\
0.15\end{array}$ & $\begin{array}{l}19.5 \\
4.20 \\
3.55 \\
0.50 \\
0.15\end{array}$ & $\begin{array}{l}18.7 \\
2.44 \\
1.79 \\
0.50 \\
0.15\end{array}$ & $\begin{array}{l}0.5 \\
0 .\end{array}$ \\
\hline 2.3 & $\begin{array}{r}0.0 \\
2.25 \\
2.25 \\
0.00 \\
0.00\end{array}$ & $\begin{array}{r}0.0 \\
2.25 \\
2.25 \\
0.00 \\
0.00\end{array}$ & $\begin{array}{r}0.0 \\
2.25 \\
2.25 \\
0.00 \\
0.00\end{array}$ & $\begin{array}{r}0.0 \\
2.25 \\
2.25 \\
0.00 \\
0.00\end{array}$ & $\begin{array}{r}0.0 \\
2.25 \\
2.25 \\
0.00 \\
0.00\end{array}$ & $\begin{array}{r}0.0 \\
2.25 \\
2.25 \\
0.00 \\
0.00\end{array}$ & $\begin{array}{r}0.0 \\
2.25 \\
2.25 \\
0.00 \\
0.00\end{array}$ & $\begin{array}{r}0.1 \\
2.25 \\
2.25 \\
0.00 \\
0.00\end{array}$ & $\begin{array}{r}0.1 \\
2.25 \\
2.25 \\
0.00 \\
0.00\end{array}$ & $\begin{array}{r}0.0 \\
0.00 \\
0.00 \\
0.00 \\
0.00\end{array}$ & $\begin{array}{r}0.0 \\
0.00 \\
0.00 \\
0.00 \\
0.00\end{array}$ & 0.0 \\
\hline
\end{tabular}

1097

TOTAL MCf

$1,342,619$

$1,342,619$

$1,049,097$

$2,000,657$

$1,732,708$
$1,049,097$

$2,000,657$

$1,732,708$

YTD
TOTAL Maf

(2)

1) Formerly Pennsylvania Gas \& Water Company.

Formerly known as Altresco Pittsfield, L.P.

Formerly known as Alliresco Pittsfield, L.P.
4) Volumes often are loo small to be shown on a monthly basis. 
VOLUME AND PRICE REPORT

\section{Long-Term Imports}

Volumes (MMcf/d) \& Prices (\$MMBTU) of Natural

Gas Imported During the Past 12 Months 04/01/96 - 03/31/97

Long-Term Importer

POWER CITY PARTNERS, L.P.

(Husky Oil Operations Ltd.)

AVG Daily Quantity

Total Price (Massena. New York)

Commodity Component

Demand Component

Reservation Fee Component

PROGAS U.S.A. INC. (1)

(ProGas Limited)

AVG Daily Quantity

Total Price (Noyes. Minnesota)

Commodity Component

Demand Component

Reservation Fee Component

PROGAS U.S.A. INC. (2)

(ProGas Limited)

AVG Daily Quantity

Total Price (Noyes. Minnesota)

Commodity Component

Demand Componen

Reservation Fee Component

PROGAS U.S.A. INC. (3)

(ProGas Limited)

AVG Daily Quantity

Total Price (Noyes, Minnesota)

Commodity Component

Demand Component

Reservation Fee Component

PROGAS U.S.A. INC. (4)

(ProGas Limited)

AVG Daily Quantity

Total Price (Noyes, Minnesota)

Commodity Component

Demand Component

Reservation Fee Component
Auth

$1996 \quad 1996 \quad 1996$

Apr. May Jun 19961996

21.0

$\begin{array}{rrr}16.8 & 7.9 & 16.3 \\ \frac{3.42}{2.05} & \frac{4.08}{2.05} & \frac{3.53}{2.05} \\ 1.37 & 2.03 & 1.48 \\ 0.00 & 0.00 & 0.00\end{array}$

16.7
3.44
2.05
1.39
0.00

\begin{tabular}{l}
16.8 \\
3.43 \\
\hline 2.05 \\
1.38 \\
0.00
\end{tabular}

16.6

$0.00 \quad 0.00$

85.0

$\begin{array}{ll}85.0 & 85.0 \\ \frac{2.18}{1.44} & \frac{2.07}{1.33} \\ 0.74 & 0.74\end{array}$

$\begin{array}{ll}0.74 & 0.74 \\ 0.00 & 0.00\end{array}$

15.0

$\begin{array}{lll}14.8 & 14.8 & 12.3 \\ \frac{2.90}{2.00} & \frac{2.40}{1.65} & \frac{2.45}{1.55} \\ 0.90 & 0.75 & 0.90 \\ 0.00 & 0.00 & 0.00\end{array}$

15.0

$\frac{2.76}{2.02}$

0.74
0.00

15.0
2.46
1.58

$\frac{2.46}{1.58}$

0.88
0.00

14.7
2.02
1.27
0.75
0.00

14.7
2.05
1.30
0.75
0.00

13.8
2.79
1.98
0.81
0.00

\begin{tabular}{l}
12.6 \\
3.72 \\
\hline 2.83 \\
0.89 \\
0.00
\end{tabular}

$\begin{array}{r}13.9 \\ 4.36 \\ \hline\end{array}$

$\frac{4.36}{3.46}$

0.90
0.00

$\frac{2.97}{2.07}$

$0.90 \quad 0.90$

10.3

\begin{tabular}{rr}
0.0 & 0.0 \\
0.00 & 0.00 \\
\hline 0.00 & 0.00 \\
0.00 & 0.00 \\
0.00 & 0.00
\end{tabular}

0.0

$\frac{0.00}{0.00}$

0.0

$\frac{0.00}{0.00}$

0.0

$\begin{array}{ll}0.00 & 0.00 \\ 0.00 & 0.00\end{array}$

0.00
0.00

7.8

\begin{tabular}{rrr}
0.0 & 0.0 & \\
0.00 & 0.00 & 0.0 \\
\hline 0.00 & 0.00 & 0.00 \\
0.00 & 0.00 & 0.00 \\
0.00 & 0.00 & 0.00
\end{tabular}

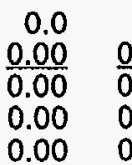

\begin{tabular}{ll}
0.0 & 0.0 \\
0.00 & 0.00 \\
\hline 0.00 & 0.00 \\
0.00 & 0.00 \\
0.00 & 0.00
\end{tabular} $\begin{array}{ll}0.0 & 0.0\end{array}$

$\frac{0.00}{0.00} \quad 0.00$ $\begin{array}{ll}0.00 & 0.00 \\ 0.00 & 0.00\end{array}$
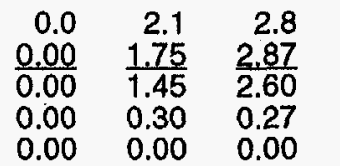

2.8
3.90
3.67
0.23
0.00

\begin{tabular}{r}
2.8 \\
3.82 \\
\hline 3.56 \\
0.26 \\
0.00
\end{tabular}

3.4
2.31
2.10

1.95

$0.21 \quad 0.23$ 
VOLUME AND PRICE REPORT

Long-Term Imports

Volumes (MMct/d) \& Prices (\$MMBTU) of Natural

Gas Imported During the Past 12 Months 04/01/96 - 03/31/97

Long-Term Importer

$\begin{array}{lllllll}\text { Auth } & 1996 & 1996 & 1996 & 1996 & 1996 & 1996\end{array}$

Vols. Apr May. Jun.

Jul.

Aug.

Sep.

PROGAS U.S.A. INC. - (NATURAL GAS CLEARINGHOUSE)

(ProGas Limited)

AVG Daily Quant

Total Price (Port of Morgan. MT)

30.0

$\begin{array}{ll}29.4 & 29.56 \\ 2.56 & 2.0 \\ 0.47 & 0.4 \\ 0.00 & 0.0\end{array}$

29.5
2.04
1.60
0.44
0.00

29.4

1996

1996

1996

Demand Component

Reservation Fee Component

PROGAS U.S.A. INC. - (TENASKA GAS COMPANY)

(ProGas Limited)

AVG Daily Quantity

Total Price (Port of Morgan, MT)

20.0

Commodity Component

Reservation Fee Component

\begin{tabular}{lll}
19.6 & 19.6 & 14.3 \\
2.55 & 2.05 & 2.35 \\
\hline 2.05 & 1.56 & 1.66 \\
0.50 & 0.49 & 0.69 \\
0.00 & 0.00 & 0.00
\end{tabular}

19.6
2.39
1.89
0.50
0.00

19.7

0.00

0.0

$\begin{array}{lll}17.0 & 16.9 & 17 \\ 2.29 & 2.30 & 2.31 \\ 0.73 & 0.73 & 0.73 \\ 1.56 & 1.57 & 1.58 \\ 0.00 & 0.00 & 0.00\end{array}$

10.0

$\begin{array}{llll}10.0 & 10.0 & 9.9 & 10.0 \\ \frac{2.74}{1.80} & \frac{2.67}{1.72} & \frac{2.60}{1.64} & \frac{2 .}{1.62} \\ 0.94 & 0.95 & 0.96 & 0.95 \\ 0.00 & 0.00 & 0.00 & 0.00\end{array}$

$10.0 \quad 10.0$

$\begin{array}{lll}0.0 & 10.0 & 10.0\end{array}$

(Alberta NE Gas (TCGS Ltd. 1))

AVG Daily Quantity

Total Price (Waddington, NY)

Demmodity Component

Reservation Fee Component

0.00

RENAISSANCE ENERGY (U.S.) INC. (2)

(Renaissance Energy Limited)

AVG Daily Quantity

Total Price (Port of Morgan, MT)

Commodity Component

Demand Component

Reservation Fee Component

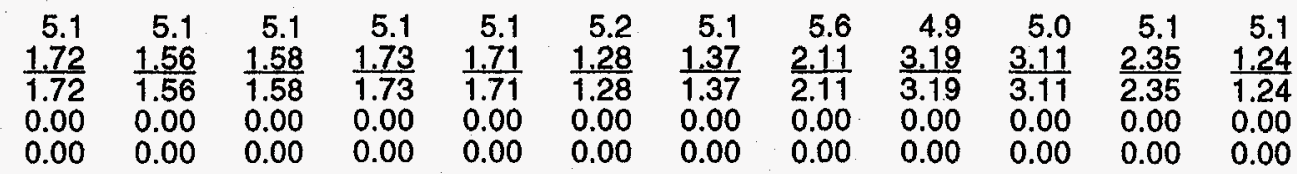

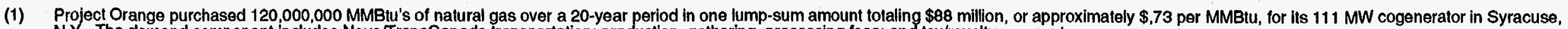

(2) . . The demand component lncludes Nove/T ansCanada transportation; production, gathering, processing fees; and tax/royally payments. 
VOLUME AND PRICE REPORT

\section{Long-Term Imports}

Volumes (MMct/d) \& Prices (\$MMBTU) of Natural

Gas Imported During the Past 12 Months 04/01/96 - 03/31/97
Long-Term Importer

RENAISSANCE ENERGY (U.S.) INC. (1)

(Renaissance Energy Limited)

AVG Daily Quantity

Total Price (Niagara Falls, NY)

Demand Component

Reservation Fee Component
Commodity Component

ROCHESTER GAS AND ELECTRIC CORPORATION (2)

(Renaissance Energy Limited)

AVG Daily Quantity

10.0

Total Price (Grand Island, NY)

Commodity Component

Demand Component

Reservation Fee Component

SALMON RESOURCES LTD.-ENRON GAS MARKETING

(Shell Canada Limited)

AVG Daily Quantity

Total Price (Port of Morgan, MT)

Commodity Component

Demand Component

Reservation Fee Component
9.8

\begin{tabular}{rr}
9.6 & 9.5 \\
1.88 & $\frac{1.68}{1.68}$ \\
\hline 1.88 & 0 \\
0.00 & 0.00 \\
0.00 & 0.00
\end{tabular} $\begin{array}{rr}9.7 & 9.7 \\ 1.67 & 1.74\end{array}$

$\frac{1.67}{1.67}$

0.00
0.00

\begin{tabular}{r}
9.7 \\
1.74 \\
\hline 1.74 \\
0.00 \\
0.00
\end{tabular}
9.7
$\frac{1.97}{1.97}$
0.00
0.00

9.7

$\frac{2.48}{2.48}$

2.48
0.00
0.00

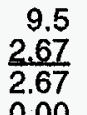

9.7
2.02

$\frac{2.02}{2.02}$

0.00

0.00

0.00

\begin{tabular}{r}
9.7 \\
1.43 \\
\hline 1.43 \\
0.00 \\
0.00
\end{tabular}

SALMON RESOURCES LTD.-MIDAMERICAN ENERGY COMPANY (3)

(Shell Canada Limited)

AVG Daily Quantity

Total Price (Port of Morgan. MT)

Commodity Component

Demand Component

Reservation Fee Component

20.5

\begin{tabular}{ll}
20.3 & 20.3 \\
1.82 & 1.51 \\
\hline 1.82 & 1.51 \\
0.00 & 0.00 \\
0.00 & 0.00
\end{tabular}

20.3
1.53
1.53
0.00

$\begin{array}{lll}0.00 & 0.00 & 0.00\end{array}$

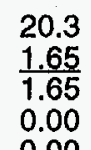

20.3

$\frac{1.65}{1.65}$

0.00
0.00

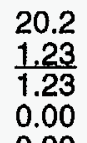

20.3

$\frac{1.31}{1.31}$

1.31
0.00

20.2

$\frac{2.13}{2.13}$

0.00
0.00

20.2

$\frac{3.14}{3.14}$

0.00

20.2

$\frac{3.62}{3.62}$

20.3

$\frac{2.37}{2.37}$

20.3

$\frac{1.12}{1.12}$

$\begin{array}{lll}0.00 & 0.00 & 0.00\end{array}$

SAN DIEGO GAS \& ELECTRIC COMPANY

Bow Valley Industries Ltd.)

AVG Daily Quantity

Total Price (Eastport, Idaho)

Commodity Component

Demand Component

Reservation Fee Component

\begin{tabular}{|c|c|c|c|c|c|c|c|c|c|c|c|c|}
\hline 4.9 & $\begin{array}{r}0.0 \\
0.00 \\
0.00 \\
0.00 \\
0.00\end{array}$ & $\begin{array}{r}0.0 \\
0.00 \\
0.00 \\
0.00 \\
0.00\end{array}$ & $\begin{array}{r}0.0 \\
0.00 \\
0.00 \\
0.00 \\
0.00\end{array}$ & $\begin{array}{r}0.0 \\
0.00 \\
0.00 \\
0.00 \\
0.00\end{array}$ & $\begin{array}{r}0.0 \\
0.00 \\
0.00 \\
0.00 \\
0.00\end{array}$ & $\begin{array}{r}0.0 \\
0.00 \\
0.00 \\
0.00 \\
0.00\end{array}$ & $\begin{array}{r}0.0 \\
0.00 \\
0.00 \\
0.00 \\
0.00\end{array}$ & $\begin{array}{r}0.0 \\
0.00 \\
0.00 \\
0.00 \\
0.00\end{array}$ & $\begin{array}{r}0.0 \\
0.00 \\
0.00 \\
0.00 \\
0.00\end{array}$ & $\begin{array}{r}0.0 \\
0.00 \\
0.00 \\
0.00 \\
0.00\end{array}$ & $\begin{array}{r}0.0 \\
0.00 \\
0.00 \\
0.00 \\
0.00\end{array}$ & $\begin{array}{r}0.0 \\
0.00 \\
0.00 \\
0.00 \\
0.00\end{array}$ \\
\hline
\end{tabular}

Auth

Auth
Vols

2.8

\begin{tabular}{lrr}
2.8 & 2.8 \\
$\frac{3.35}{2.44}$ & 2.76 \\
\hline 0.91 & 1.88 & \\
0.88 & 2.88 & 0.9 \\
0.00 & 0.00 & 0.0
\end{tabular}

1996

Jul.

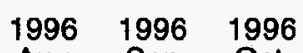

Aug.

Sep.

$96 \quad 1996$

t.

1996
De.
$1997 \quad 1997 \quad 1997$

\begin{tabular}{|r}
2.8 \\
\hline 3.26 \\
\hline 2.38 \\
0.88 \\
\hline \\
0.00
\end{tabular}

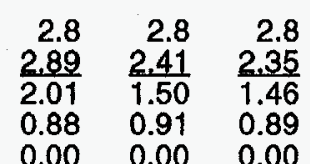

0.00

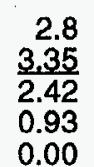

$\begin{array}{r}2.8 \\ 4.55 \\ \hline 3.66\end{array}$

0.89

0.00

2.8
4.72
3.83

0.89

$\begin{array}{r}2.8 \\ 3.64 \\ \hline .65\end{array}$

2.65

0.99

$\frac{2.30}{1.41}$

0.89

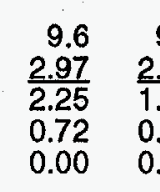

$\begin{array}{rr}0.0 & 0.0\end{array}$

$\frac{0.00}{0.00} \quad 0.00$

0.00

0.00

0.00

\begin{tabular}{rrr}
0.0 & 0.0 & 0.0 \\
0.00 & $\underline{0.00}$ & 0.00 \\
\hline 0.00 & 0.00 & 0.00 \\
0.00 & 0.00 & 0.00 \\
0.00 & 0.00 & 0.00
\end{tabular}

$\begin{array}{llll}0.00 & 0.00 & 0.00 & 0.00\end{array}$

\section{$0.0 \quad 10.0$}

$\underline{0.00}$

0.00

0.00 $\frac{4.32}{4.32}$

0.00
0.00
$10.8 \quad 11.2$

$\frac{4.90}{4.90} \quad \frac{3.49}{3.49}$

4.90
0.00

$\begin{array}{ll}0.00 & 0.00 \\ 0.00 & 0.00\end{array}$
11.1
2.75
2.75
0.00
0.00
9.7
1.74
1.74
0.00
0.00 \begin{tabular}{r}
9.6 \\
1.53 \\
\hline 1.53 \\
0.00
\end{tabular}

\begin{tabular}{rr}
9.6 & 9.7 \\
53 & 1.58 \\
\hline .53 & 1.58 \\
\hline 00 & 0.00
\end{tabular}

0.00
865,321

865,321

YTD

1Q97
TOTAL

252,000

252,000

992,471

992,471

52,000
92,471
9,321

(1) Renaissance resells these volumes to Delmarva Power \& Light Company.

3) Effective November 1, 1996, contract with Westcoast was terminated. Beginning November 1, 1996, RG\&E has a long-term peaking supply arrangement with Renaissance (9.9 MMcf/d). Midwest Power Systems Inc. and lowa-llinoi
and lowa-Illinois Gas and Electric Company. 
$0 S L^{\prime} \angle 19^{\prime} L$

OSL'LL9'L

\begin{tabular}{ll}
$00^{\circ} 0$ & 00.0 \\
$9 L^{\circ} L$ & $91 . L$ \\
$9 E^{\prime} L$ & $18 . L$ \\
\hline $9^{\circ} Z$ & $\frac{26^{\circ} Z}{2 L}$
\end{tabular}

8.9L 0.LZ
000
$\angle 1$.

$\frac{96 .}{\varepsilon ! \cdot}$
$00 \%$
91.5

$\frac{09^{\circ} L}{9 L^{\circ} \mathrm{C}}$

$9 L 2$
$\varepsilon ' L$
000

$82 !$

टt'

000

Lद'L

द'LI

$00^{\circ} 0$
+1.1

टहंเ

962
0.81

SL.'

$\frac{9 \varepsilon^{\prime} L}{1 G^{\prime 2}}$

$00^{\circ} 0$

GL:

$00 \%$

S1:1

00.0

¿l.

09.1

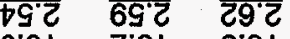

ट'9l S'9l

\begin{tabular}{|c|c|c|c|c|c|c|c|c|c|c|}
\hline $\begin{array}{l}00^{\circ} 0 \\
88^{\circ} 0 \\
\angle \nabla^{\circ} Z \\
\overline{S E}^{\circ} \varepsilon \\
7^{\circ} c\end{array}$ & $\begin{array}{l}00^{\circ} 0 \\
86^{\circ} 0 \\
9 \varepsilon^{\circ} Z \\
7 \varepsilon^{\prime} \varepsilon \\
9^{\circ} \angle t\end{array}$ & $\begin{array}{l}00^{\circ} 0 \\
68^{\circ} 0 \\
9 \nabla^{\circ} \mathcal{Z} \\
\varepsilon^{\prime} \varepsilon \\
9^{\circ} 9 t\end{array}$ & $\begin{array}{l}00^{\circ} 0 \\
88^{\circ} 0 \\
9 t^{\circ} \tau \\
7 \varepsilon^{\prime} \varepsilon \\
0^{\circ} 9 t\end{array}$ & $\begin{array}{l}00^{\circ} 0 \\
\varepsilon 6^{\circ} 0 \\
\left\llcorner\sigma^{\circ} \mathrm{C}\right. \\
b \varepsilon^{\circ} \varepsilon \\
S^{\prime} 0 \varepsilon\end{array}$ & $\begin{array}{l}00^{\circ} 0 \\
68^{\circ} 0 \\
Z \varepsilon^{\circ} Z \\
C^{\prime} Z \\
8^{\circ} 0 \phi\end{array}$ & $\begin{array}{l}00^{\circ} 0 \\
16^{\prime} 0 \\
0 \varepsilon^{\circ} Z \\
L^{\prime} Z \\
Z^{\prime} \varepsilon \phi\end{array}$ & $\begin{array}{l}00^{\circ} 0 \\
88^{\circ} 0 \\
t \varepsilon^{\circ} Z \\
Z^{\prime} \varepsilon \\
9^{\circ} \varepsilon t\end{array}$ & $\begin{array}{l}00^{\circ} 0 \\
88^{\circ} 0 \\
\varepsilon \varepsilon^{\prime} Z \\
L^{\prime} \varepsilon \\
\varepsilon^{\prime} \varepsilon t\end{array}$ & $\begin{array}{l}00^{\circ} 0 \\
16^{\circ} 0 \\
0 \varepsilon^{\circ} Z \\
L^{\prime} Z \\
L^{\prime} Z\end{array}$ & $\begin{array}{l}00^{\circ} 0 \\
88^{\circ} 0 \\
\varepsilon \varepsilon^{\prime} Z \\
L^{\prime} \varepsilon \\
0^{\circ} \varepsilon \Rightarrow\end{array}$ \\
\hline
\end{tabular}

$\left\llcorner 9^{\prime} 08 L^{\prime b}\right.$

$169^{\prime} 08 L^{\prime \prime}$

¿' 9

$9^{\circ} \angle t$

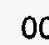

\begin{tabular}{ll}
$00^{\circ} 0$ & 00.0 \\
$00^{\circ} 0$ & $00^{\circ} 0$ \\
$00^{\circ} 0$ & $00^{\circ} 0$ \\
\hline $00^{\circ} 0$ & $00^{\circ} 0$ \\
$0^{\circ} 0$ & 0.0
\end{tabular}

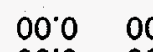

$00^{\circ} 00^{\circ}$

$\frac{000}{00^{\circ} 0}$

00.0

$00^{\circ} 0$

$00^{\circ} 0$

$00 \%$

$00^{\circ} 0$

$00^{\circ} 0$

$\frac{00^{\circ}}{00^{\circ} 0}$

$\frac{00.0}{00^{\circ} 0}$

$00^{\circ} 0$

$\frac{00^{\circ}}{00^{\circ} 0}$

$00^{\circ} 0$

\begin{tabular}{l}
00.0 \\
00.0 \\
\hline 00.0
\end{tabular}

$00^{\circ} 0$
$00^{\circ} 0$

\begin{tabular}{l}
$00^{\circ} 0$ \\
$00^{\circ} 0$ \\
\hline $00^{\circ} 0$
\end{tabular}

$00 \%$
$00 \%$

$00^{\circ} 0 \quad 00^{\circ}$

$00^{\circ} 0 \quad 00^{\circ} 0$

$000 \overline{00}$

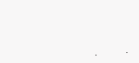

$\angle 0 Z^{\prime} \varepsilon \varepsilon 6^{\prime}$

LOZ'ยะ6'เ

\begin{tabular}{ll}
$00^{\circ} 0$ & $00^{\circ}$ \\
$00^{\circ} 0$ & $00^{\circ}$ \\
$96^{\circ} 0$ & $\angle 8^{\circ}$ \\
\hline $9^{\circ} 0$ & $\angle 8$ \\
$t^{\circ} \cdot{ }^{\circ} \mathrm{C}$ & $9^{\circ} L$
\end{tabular}

0

0

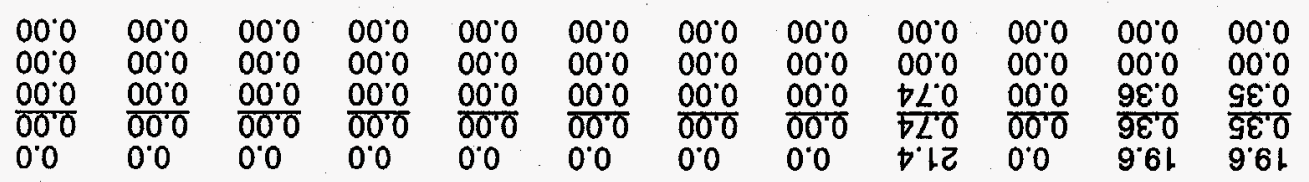

!़W TVIOI

aㅅ․
IJW TVIOI
L6OL
गलW
$\angle 661$

\begin{tabular}{|c|c|}
\hline $\begin{array}{l}00^{\circ} 0 \\
00^{\circ} 0 \\
8 t^{\circ} \varepsilon \\
8 b^{\circ} \varepsilon \\
t^{\circ} L Z\end{array}$ & $\begin{array}{l}00^{\circ} 0 \\
00^{\circ} 0 \\
66^{\circ} 2 \\
66^{\circ} \mathrm{Z} \\
9^{\circ} 12\end{array}$ \\
\hline $\begin{array}{l}00^{\circ} 0 \\
00^{\circ} 0 \\
00^{\circ} 0 \\
00^{\circ} 0 \\
0^{\circ} 0\end{array}$ & $\begin{array}{l}00^{\circ} 0 \\
00^{\circ} 0 \\
00^{\circ} 0 \\
00^{\circ} 0 \\
0^{\circ} 0\end{array}$ \\
\hline
\end{tabular}

$00 \%$

\begin{tabular}{l}
$00^{\circ}$ \\
$\angle 60$ \\
\hline
\end{tabular}

$\frac{\angle 6}{260}$

D.LC $\begin{array}{llllll}00^{\circ} 0 & 00^{\circ} 0 & 00^{\circ} 0 & 00^{\circ} 0 & 00^{\circ} 0 & 00^{\circ} 0 \\ 00^{\circ} 0 & 00^{\circ} 0 & 00^{\circ} 0 & 00^{\circ} 0 & 00^{\circ} 0 & 00^{\circ} 0\end{array}$

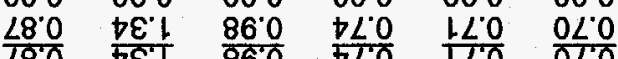

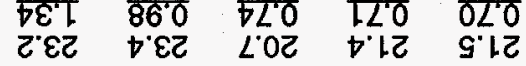

$0.6 \mathrm{~L}$

quavodwo $\theta \theta y$ uolteneser Leuoduos puewed jueuoduso Kupoumos

( (रN UolbuाppeM)

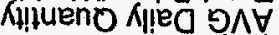

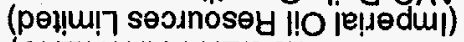
d' 7 'SH

juevoduo $\theta \theta-$ uolpenesey luenodüo puewea juevoduos Kupowmos

0.79

(रN $u$ ! वस्व

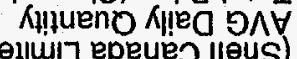

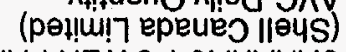

d`ר'S

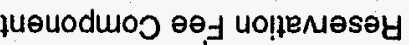
tuenoduso puemea queuodmo Kupoumos

(0पष्या मodise

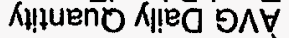

6.9

(p) 7 soomosey juum

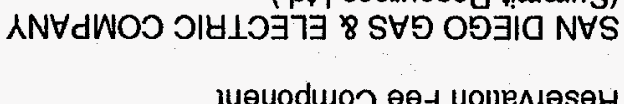

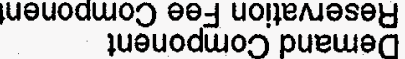
juevodwos Kupomulos

$L ' L C$

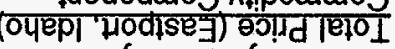

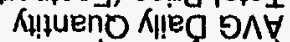

('piา suo!lededo $1 ! O K y s n H$ )

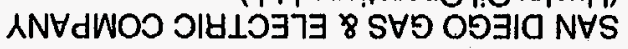

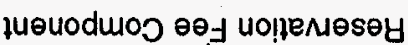
ueuoduros puemer juevodulos Kupoumos

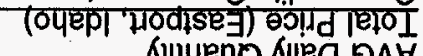

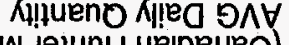

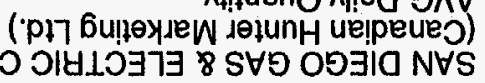

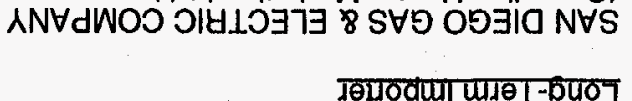

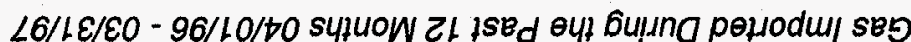

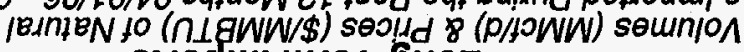
spodul wdoL-6uo7

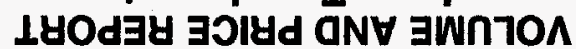


VOLUME AND PRICE REPORT

Long-Term Imports

Volumes (MMct/d) \& Prices (\$MMBTU) of Natural

Gas Imported During the Past 12 Months 04/01/96 - 03/31/97

Long-Term Importer

SELKIRK COGEN PARTNERS, L.P.

(PanCanadian Petroleum Ltd.)

AVG Daily Quantity

Total Price (Waddington. NY)

Commodity Component

Demand Component

Reservation Fee Component

SELKIRK COGEN PARTNERS, L.P.

(Paramount Resources Ltd.)

AVG Daily Quantity

Total Price (Waddington. NY)

Commodity Component

Demand Component

Reservation Fee Component

SELKIRK COGEN PARTNERS, L.P.

(Producers Marketing, Ltd.)

AVG Daily Quantity

Total Price (Waddington, NY)

Commodity Component

Demand Component

Reservation Fee Component

SITHE/INDEPENDENCE POWER PARTNERS, L.P.

(Enron Power Services Inc.)

$\begin{array}{llllll}\text { Auth. } & 1996 & 1996 & 1996 & 1996 & 1996\end{array}$

Vols.

Apr.

May.

19.0

$\begin{array}{lll}16.5 & 15.2 & 16.0 \\ \frac{2.61}{1.50} & \frac{2.58}{1.44} & 2.51 \\ 1.38 \\ 1.11 & 1.14 & 1.13 \\ 0.00 & 0.00 & 0.00\end{array}$

23.0

$\begin{array}{rrr}12.6 & 9.9 & 10.0 \\ 2.63 & 2.60 & 2.47 \\ 1.91 & 1.87 & 1.73 \\ 0.72 & 0.73 & 0.74 \\ 0.00 & 0.00 & 0.00\end{array}$

0.00

17.0

$\begin{array}{lll}14.7 & 13.6 & 14 . \\ 2.55 & 2.52 & 2.4 \\ 1.54 & 1.48 & 1.4 \\ 1.01 & 1.04 & 1.04 \\ 0.00 & 0.00 & 0.00\end{array}$

$2 \quad 15.3$

0.00
AVG Daily Quantity

Total Price (Grand Island. NY)

Commodity Component

Demand Component

Reservation Fee Component

$\begin{array}{lll}28.5 & 28.6 & 28.5 \\ \frac{3.23}{2.51} & \frac{3.21}{2.51} & \frac{3.23}{2.51} \\ 0.72 & 0.70 & 0.72 \\ 0.00 & 0.00 & 0.00\end{array}$

0.00

$\begin{array}{lll}15.0 & 15.0 & 15.0 \\ \frac{2.86}{2.40} & \frac{2.71}{2.26} & \frac{2.78}{2.32} \\ 0.46 & 0.45 & 0.46\end{array}$

0.00

0.00

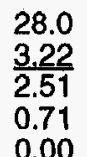

28.0

27.9

28.0
$\frac{3.22}{2.51}$

28.0
$\frac{3.25}{2.51}$
0.74

\begin{tabular}{l}
28.0 \\
3.22 \\
\hline 2.51 \\
0.71
\end{tabular}

27.9
$\frac{3.53}{2.82}$
0.71

$\frac{3.61}{2.82} \quad \frac{3.53}{2.82}$

$\begin{array}{ll}0.79 & 0.71 \\ 0.00 & 0.00\end{array}$

SOUTHEASTE

AVG Dally Ouantity

Total Price (Noyes, Minnesota)

Demand Component

Reservation Fee Component

\begin{tabular}{ll}
10.0 & 15.0 \\
3.29 & 2.80 \\
\hline 2.62 & 2.35 \\
0.67 & 0.45 \\
0.00 & 0.00
\end{tabular}

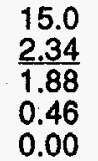

15.0
2.37
1.92
0.45
0.00
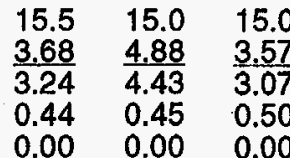

15.0

0.00

2.34
1.40
1.04

16.1
2.40
1.36

$\frac{2.40}{1.36}$

0
6

15.3
2.28
1.25
1.03
0.00

14.9
2.35
1.31
1.04
0.00

15.4
2.69

$\frac{2.69}{1.64}$

1.64
1.05
0.00

$\frac{3.13}{2.07}$

\begin{tabular}{ll}
27.9 & 27.9 \\
\hline &
\end{tabular}

990,471

990,471

$1,439,785$

$1,439,785$

\begin{tabular}{l}
15.0 \\
4.88 \\
\hline 4.43 \\
0.45 \\
0.00
\end{tabular}

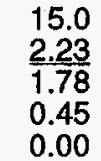

(

YTD

$1,617,750$ 
VOLUME AND PRICE REPORT

\section{Long-Term Imports}

Volumes (MMcf/d) \& Prices (\$MMBTU) of Natural

Gas Imported During the Past 12 Months 04/01/96 - 03/31/97
Leng-Term Importer

SOUTHERN CALIFORNIA EDISON COMPANY

(AEC Oil \& Gas Company)

AVG Daily Quantity

Total Price (Eastpont, Idaho)

Commodity Component

Demand Component

Reservation Fee Component

SOUTHERN CALIFORNIA EDISON COMPANY

(Imperial Oil Resources Limited)

AVG Daily Quantity

Total Price (Eastport. Idaho)

Commodity Component

Demand Component

Reservation Fee Component

SOUTHERN CALIFORNIA EDISON COMPANY

(Shell Canada Limited)

AVG Daily Quantity

Total Price (Eastport. Idaho)

Commodity Component

Demand Component

Reservation Fee Component

SOUTHEAN CALIFORNIA EDISON COMPANY (TCGS Ltd.)

AVG Daily Quantity

Total Price (Eastport. Idaho)

Commodity Component

Demand Component

Reservation Fee Component

1996

19961996

Apr. May Jun.

51.8

\begin{tabular}{lll}
34.9 & 34.9 & \\
1.45 & $\frac{1.74}{1.79}$ & 0. \\
\hline 1.39 & 1.68 & 0.0 \\
0.06 & 0.06 & 0.0 \\
0.00 & 0.00 & 0.0
\end{tabular}

\begin{tabular}{r}
0.0 \\
0.00 \\
\hline 0.00 \\
0.00 \\
0.00
\end{tabular}

0.0
0.00
0.00
0.00
0.00

35.1
1.54

1996

1996

0.00

0.00

\begin{tabular}{ll}
24.9 & 24.9 \\
1.45 & $\frac{1.74}{1.39}$ \\
\hline 1.68 \\
0.06 & 0.06
\end{tabular}

0.0

0.00
0.00
0.00

$0.00 \quad 0.00$

51.0

\begin{tabular}{rr}
49.9 & 49.9 \\
1.33 & 1.64 \\
\hline
\end{tabular}

$\frac{1.33}{1.27} \quad \frac{1.64}{1.58}$

0.06

0.00

0.06
0.00

$\begin{array}{r}49.9 \\ 1.75 \\ \hline\end{array}$

$\frac{1.75}{1.69}$
0.06

0.06
0.00

1.32

1.26
0.06

0.06
0.00

\begin{tabular}{r}
0.0 \\
0.00 \\
\hline 0.00 \\
0.00 \\
0.00
\end{tabular}

0.00
0.00

Aug.

Sep.

1996

Nov.

1996

\begin{tabular}{ll}
34.9 & 34.9 \\
1.45 & $\frac{1.74}{1.68}$ \\
\hline 1.39 & 1.68 \\
0.06 & 0.06 \\
0.00 & 0.00
\end{tabular}

\begin{tabular}{r}
0.0 \\
0.00 \\
\hline 0.00 \\
0.00 \\
0.00
\end{tabular}

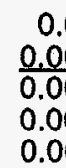

\begin{tabular}{rr}
0.0 & 24.9 \\
0.00 & 1.54 \\
\hline 0.00 & 1.48 \\
0.00 & 0.06 \\
0.00 & 0.00
\end{tabular}

0.0

$\frac{0.00}{0.00}$

0.00

0.00

2.2

\begin{tabular}{rrrrr}
2.2 & 2.2 & 2.2 & 2.2 & 2.2 \\
2.74 & 2.67 & 2.60 & 2.56 & $\frac{2.44}{1.52}$ \\
\hline 1.83 & 1.74 & 1.67 & 1.63 & 0.92 \\
0.91 & 0.93 & 0.93 & 0.93 & 0.92 \\
0.00 & 0.00 & 0.00 & 0.00 & 0.00
\end{tabular}

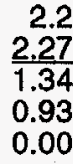

2.2
2.27
1.34
0.93
0.00

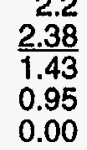

2.2
3.27
2.32

$\frac{3.27}{2.32}$
0.95

$\begin{array}{ll}0.95 & 1.07 \\ 0.00 & 0.00\end{array}$

Reservation Fee Component $\begin{array}{r}2.2 \\ 3.88 \\ \hline 2.94\end{array}$

2.94
0.94

0.94
0.00
$1997 \quad 1997$

Feb. Mar.

1Q97

TOTAL Mcf

$1,096,135$

0.0
0.00
0.00

$\frac{0.00}{0.00}$

0.00

0.00

0.01

0.00

782,966

782,966

$\begin{array}{ll}0.00 & 0.00\end{array}$

$\frac{0.00}{0.00} \quad 0.00$

0.00

$0.00 \quad 0.00$ 
<smiles>[B]=C=[Fe]</smiles><smiles>O</smiles>

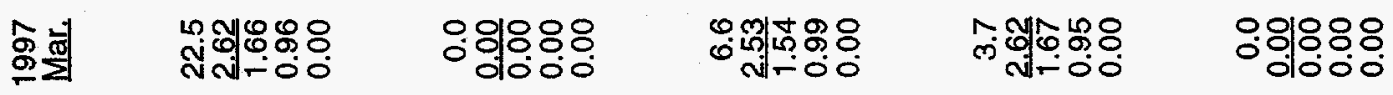

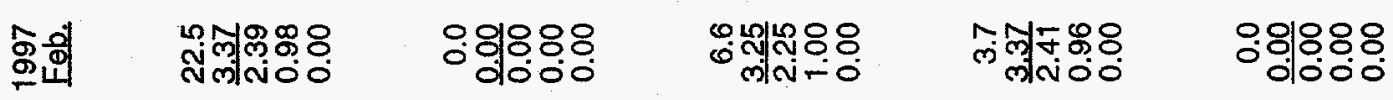

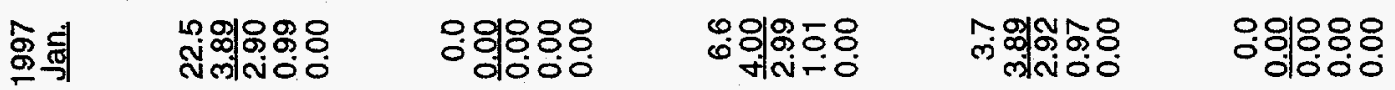

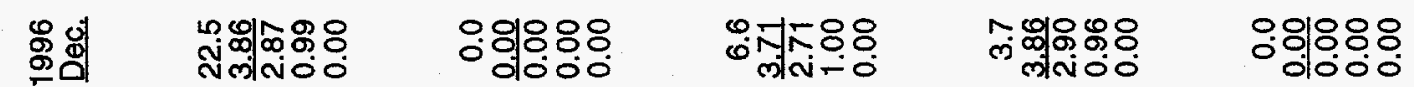

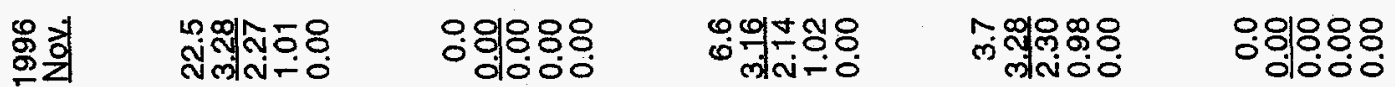

잉융ㅇㅇㅇㅇㅇ

원융ㅇㅇㅇ

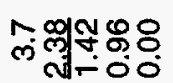

웅잉웅웅

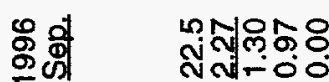

웅영ㅇㅇㅇㅇㅇㅇ

ํㅐㄲ용ㅇㅇ

लํํำ

잉영ㅇㅇㅇ

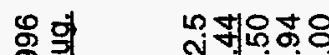

영ㅇㅇㅇㅇㅇㅇ

ตํํำㅇํㅇㅇㅠ

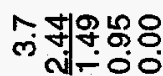

영잉융융

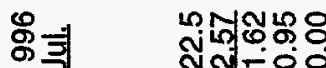

영영융유.

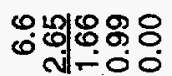

लํํํํํㅇㅇㅇㅇㅇㅇ

잉잉ㅇㅇㅇ

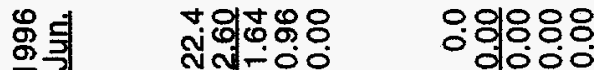

영용ㅇㅇ

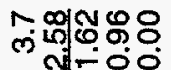

พ̛ํํํ요융

รัส

워ำํํ유.

ํํำ용ㅇㅇ

लํํำ:

하워ํㅇㅇㅇㅇ

男훈

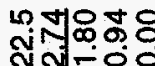

영용유

땡용ㅇㅇ

लํㅝ융유

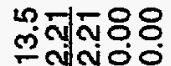

趋哭

$$
\stackrel{0}{10}
$$

$\stackrel{n}{\circ}$

$\stackrel{\bullet}{0}$

คे

$\stackrel{8}{\dot{q}}$
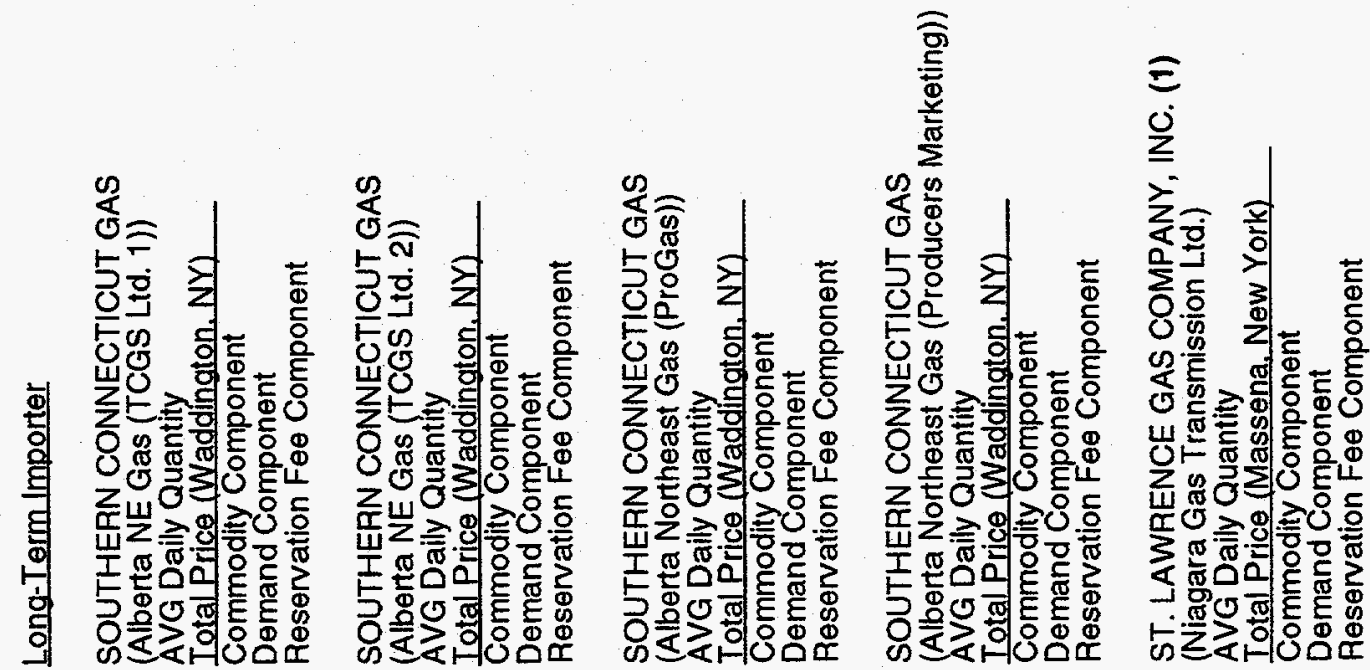


\section{VOLUME AND PRICE REPORT}

\section{Long-Term Imports}

Volumes (MMcfid) \& Prices (\$MMBTU) of Natural

Gas Imported During the Past 12 Months 04/01/96 - 03/31/97

Long-Term Importer

ST. LAWRENCE GAS COMPANY, INC.

(TCGS Ltd.)

AVG Daily Quantity

Total Price (Massena. New York)

Commodity Component

Reservation Fee Component

SUMAS COGENERATION COMPANY, L.P. (1)

AVG Daily Quantity

Total Price (Sumas, Washington)

Commodity Component

Demand Component

Reservation Fee Component

SUMAS COGENERATION COMPANY, L.P. (2)

(Westcoast Gas Services)

AVG Daily Quantity

Total Price (Sumas. Washington)

Commodity Component

Demand Component

Reservation Fee Component

TENASKA GAS COMPANY (3)

AVG Daily Quantity

Total Price (Sumas. Washington)

Commodity Componen

Demand Component

Peservation Fee Component

\section{TENASKA GAS COMPANY (4)}

(Petro-Canada Ltd.)

AVG Daily Quantity

Total Price (Sumas, Washington)

Commodity Component

Demand Component

Reservation Fee Component
Demand Component

(ENCO Gas, Ltd.)

(Husky Oil Operations Ltd.)
Auth.

$\begin{array}{llll}1996 \quad 1996 & 1996 \quad 1996\end{array}$

Apr.

20.3

\begin{tabular}{rrr}
0.0 & 0.0 & 0.0 \\
0.00 & $\frac{0.00}{0.00}$ & $\underline{0.00}$ \\
\hline 0.00 & 0.00 & 0.00 \\
0.00 & 0.00 & 0.00 \\
0.00 & 0.00 & 0.00
\end{tabular}
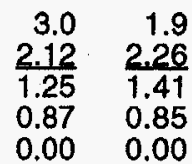

4.4
2.04
1.04
1.00
0.00

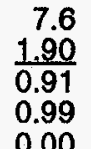

14.1
2.20
1.15
1.05
0.00

13.9
2.43
1.42
1.01
0.00

14.8
2.77
1.79
0.98
0.00

$14.7 \quad 15.3$

$1.06 \quad 0.67$

$0.00 \quad 0.00$

12.0

\begin{tabular}{rrr}
9.9 & 10.9 & 7.7 \\
2.43 & 2.43 & 2.43 \\
\hline 2.43 & 2.43 & 2.43 \\
0.00 & 0.00 & 0.00
\end{tabular}

10.2

$\begin{array}{ll}7.1 & 10.4\end{array}$

\begin{tabular}{l}
10.5 \\
2.43 \\
\hline
\end{tabular}

$0.00 \quad 0.00$

2.43
0.00

2.43
0.00

$\frac{2.43}{2.43}$

$\frac{2.43}{2.43}$

2.43
0.00

0.00

$\frac{2.43}{2.43}$

0.00

11.1
2.07
2.07
0.00

11.6
2.07

$\frac{2.07}{2.07}$
0.00

10.6
2.07
2.07

0.00

$\begin{array}{r}0.0 \\ 0.00 \\ \hline 0.00\end{array}$

$\frac{0.00}{0.00}$

0.00

$\begin{array}{lllll}12.4 & 12.4 & 12.4 & 12.4 & 12.3 \\ 129 & 129 & 129 & 129 & 1.29\end{array}$

$\frac{1.29}{0.89} \quad \frac{1.29}{0.89}$

$0.40 \quad 0.40$

$\frac{1.29}{0.89}$

0.89
0.40

12.3
$\frac{1.29}{0.89}$

12.4
1.29
0.89
0.40

12.4

11.7

$0.00 \quad 0.00$

0.00

$\begin{array}{ll}0.00 & 0.00\end{array}$

0.00

0.89

0.40
0.00

0.40
0.00

11.7
1.20

$\frac{1.20}{0.80}$

0.40
0.00

4.1

$\frac{1.20}{0.80}$

$0.80 \quad 0.00$

$0.40 \quad 0.00$

$\frac{0.00}{0.00}$

0.00
0.00

13.0

$\begin{array}{rrr}0.0 & 0.0 & 2.0 \\ 0.00 & \frac{0.00}{0.00} & \frac{4.19}{1.76} \\ 0.00 & 0.00 & 2.43\end{array}$

9.6
2.20

8.5
2.20
1.76
0.44

10.3

10.6

11.0

$0.00 \quad 0.00$

0.00

2.43

0.44

0.44
0.00

$\frac{2.20}{1.76}$

0.44
0.00

$\frac{2.20}{1.76}$

0.44

$\frac{2.20}{1.76}$

8.0
2.20
1.76
0.44

12.4
2.31
1.85
0.46

0.0

$\frac{0.00}{0.00}$

0.00

$\begin{array}{lllll}0.44 & 0.44 & 0.46 & 0.00 & 0.00 \\ 0.00 & 0.00 & 0.00 & 0.00 & 0.00\end{array}$

15.0

\begin{tabular}{rrr}
0.0 & 0.0 & 5.8 \\
0.00 & 0.00 & 2.46 \\
\hline 0.00 & 0.00 & 1.51 \\
0.00 & 0.00 & 0.95 \\
0.00 & 0.00 & 0.00
\end{tabular}

9.8
2.33
1.51
0.82
0.00

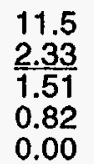

11.5
2.33
1.51
0.82
0.00

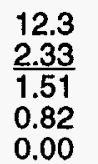

12.5
2.33
1.51
0.82
0.00

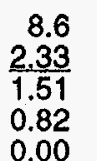

\begin{tabular}{r}
3.6 \\
2.45 \\
\hline 1.59 \\
0.86 \\
0.00
\end{tabular}

0.0

0.00

0.00
$2.85 \quad \frac{1.90}{1.23}$

$\frac{1.20}{0.80}$

$\begin{array}{ll}0.00 & 0.00\end{array}$

1097

TOTAL Mof

$1,344,357$

$1,344,357$

327,950

327,950

128,544

128,544

385,462

385,462

(1) These import volumes are transported into the United Slates on the Sumas Pipeline-U.S.A.

(2) These import volumes are transporled into the United Slates on the Sumas Pipeline-U.S.A.

(3) These import volumes are transporled Into the United Slates on the Sumas-Cascade Pipeline; demand component includes deficiency payments.

(4) These import volumes are transporled into the United States on the Sumas-Cascade Pipeline; demand component includes deficiency payments. 
VOLUME AND PRICE REPORT

Long-Term Imports

Volumes (MMcf/d) \& Prices (\$MMBTU) of Natural

Gas Imported During the Past 12 Months 04/01/96 - 03/31/97
Long-Term Importer

THE U.S. GENERAL SERVICES ADMIN. (1)

(Chief Mountain Natural Gas Coop.)

AVG Daily Quantity

Total Price (Babb. Montana)

Commodity Component

Demand Component

Reservation Fee Component

$\begin{array}{llllllllll}\text { Auth. } & 1996 & 1996 & 1996 & 1996 & 1996 & 1996 & 1996 & 1996 & 1996\end{array}$

Vols. Apr. May Jun.

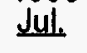

Aug.

Sep.

0.2

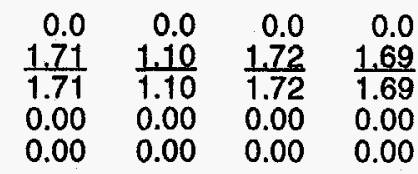

0.0
2.13
2.13
0.00
0.00

\begin{tabular}{r}
0.0 \\
1.62 \\
\hline 1.62 \\
0.00 \\
0.00
\end{tabular}

0.0
1.70
1.70

$\frac{1.70}{1.70}$

$0.00 \quad 0.00$

0.00

10.0

\begin{tabular}{rrr}
8.2 & 7.7 & 5.2 \\
2.06 & 2.06 & $\frac{2.06}{2.06}$ \\
\hline 2.06 & 2.06 & 2.06 \\
0.00 & 0.00 & 0.00 \\
0.00 & 0.00 & 0.00
\end{tabular}

5.0
2.06
2.06
0.00
0.00

7.6

(CanWest Gas Supply)

AVG Daily Quantity

Total Price (Sumas. Washington)

Demand Component

Reservation Fee Component

TRANSCO ENERGY MARKETING COMPANY (2)

(Canstates Gas Marketing)

AVG Daily Quantity

Total Price (Niagara Falls. NY)

Commodity Component

Demand Component

Reservation Fee Component

48.4

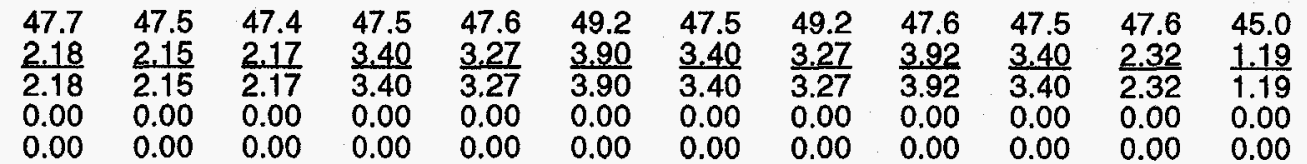

TRANSCO ENERGY MARKETING COMPANY-BG\&E

(Canstates Gas Marketing)

AVG Daily Quantity

Total Price (Niagara Falls. NY)

Commodity Component

Demand Component

Reservation Fee Component

25.0

\begin{tabular}{lll}
12.7 & 0.0 & \\
3.63 & 0.00 & 0.0 \\
\hline 2.71 & 0.00 & 0.0 \\
0.92 & 0.00 & 0.0 \\
0.00 & 0.00 & 0.0
\end{tabular}

0.0
0.00
0.00
0.00
0.00

0.0
0.00

$\frac{0.00}{0.00}$

0.00
0.00
0.00

0.0
0.00

$\frac{0.00}{0.00}$

0.0
0.00
0.00

$\frac{0.00}{0.00}$

$\begin{array}{r}0.0 \\ 0.00 \\ \hline\end{array}$

0.00
0.00

0.00

0.00

\begin{tabular}{r}
0.0 \\
0.00 \\
\hline 0.00 \\
0.00
\end{tabular}

0.0
0.00

$\frac{0.00}{0.00}$

0.00

$\begin{array}{r}0.0 \\ 0.00 \\ \hline 0.00\end{array}$

0.0
0.00

$\frac{0.00}{0.00}$

0.00
0.00
0.00

TRANSCO ENERGY MARKETING COMPANY-LILCO

(Canstates Gas Marketing)

AVG Daily Quantity

15.0

Jotal Price (Niagara Falls, NY)

Commodity Componen

Demand Component

Reservation Fee Component

$\begin{array}{llllllllllll}16.6 & 19.5 & 11.1 & 14.8 & 14.8 & 10.0 & 14.8 & 15.3 & 9.6 & 14.5 & 16.4 & 14.5 \\ \frac{3.23}{2.81} & \frac{2.57}{2.28} & \frac{2.87}{2.52} & \frac{3.68}{2.80} & \frac{3.33}{2.45} & \frac{3.31}{1.96} & \frac{3.68}{2.80} & \frac{3.33}{2.45} & \frac{3.31}{1.96} & \frac{5.10}{4.13} & \frac{3.42}{2.45} & \frac{2.89}{1.92} \\ 0.42 & 0.29 & 0.35 & 0.88 & 0.88 & 1.35 & 0.88 & 0.88 & 1.35 & 0.97 & 0.97 & 0.97 \\ 0.00 & 0.00 & 0.00 & 0.00 & 0.00 & 0.00 & 0.00 & 0.00 & 0.00 & 0.00 & 0.00 & 0.00\end{array}$

0.00

0.00

1097

IOTAL MCf

263

$4,201,159$

$4,201,159$

851,379

851,379

0

(1) Volumes often are too small to be shown on a monthly basis.

(2) Gas resold to Hopewell Cogeneration Facility and other TEMiCO customers. 


\section{VOLUME AND PRICE REPORT}

\section{Long-Term Imports}

Volumes (MMcf/d) \& Prices (\$MMBTU) of Natural Gas Imported During the Past 12 Months 04/01/96 - 03/31/97

Long-Term importer

Auth. $\quad 1996 \quad 1996 \quad 1996$

Vols.

Apr. May Jun

1996

1996

1996

1996

TRANSCO ENERGY MARKETING COMPANY-PSE\&G

(Canstates Gas Marketing)

AVG Daily Quantity

Total Price (Niagara Falls. NY)

Commodity Component

Demand Component

Reservation Fee Component

UTILICORP UNITED, INC. (1)

(ProGas Limited)

AVG Daily Quantity

Total Price (Noyes, Minnesota)

Commodity Component

Demand Component

Reservation Fee Component

UTILICORP UNITED, INC. (2)

(TCGS LId.)

AVG Daily Quantity

Total Price (Noyes. Minnesota)

Commodity Component

Demand Component

Reservation Fee Component

VALLEY GAS COMPANY

(Alberta NE Gas (TCGS Ltd. 1))

AVG Daily Quantity

Total Price (Waddington. NY)

Commodity Component

Demand Component

Reservation Fee Component

VERMONT GAS SYSTEMS INC. (3)

(TCGS Ltd.)

AVG Daily Quantity

Total Price (Highgate Spr., VT)

Commodity Component

Demand Component

Reservation Fee Component
35.0

\begin{tabular}{ll}
39.3 & 36.3 \\
3.14 & 2.70 \\
\hline 2.75 & 2.35 \\
0.39 & 0.35
\end{tabular}

$\begin{array}{ll}0.39 & 0.35 \\ 0.00 & 0.00\end{array}$

2.7

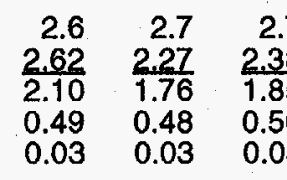

7.2

\begin{tabular}{rr}
7.0 & 7.2 \\
2.68 & 2.32 \\
\hline 2.12 & 1.79 \\
0.47 & 0.46 \\
0.09 & 0.07
\end{tabular}

7.2
2.41
1.87
0.47
0.07

7.1
2.63
2.09
0.46
0.08

7.2
2.43
1.90
0.45
0.08

\begin{tabular}{r}
6.7 \\
2.01 \\
\hline 1.43 \\
0.52 \\
0.06
\end{tabular}

\begin{tabular}{r}
7.2 \\
1.97 \\
\hline 1.44 \\
0.47 \\
0.06
\end{tabular}

\begin{tabular}{r}
7.2 \\
2.89 \\
\hline 2.29 \\
0.51 \\
0.09
\end{tabular}

7.2
$\frac{4.01}{3.39}$

3.39
0.49

0.13

$\frac{4.39}{3.75}$

0.49

$\frac{3.14}{2.51}$

$0.53 \quad 0.47$

1.0

$\begin{array}{rrr}1.0 & 1.0 & 1.0 \\ 2.74 & 2.67 & 2.60 \\ 1.80 & 1.72 & 1.64 \\ 0.94 & 0.95 & 0.9 \\ 0.00 & 0.00 & 0.00\end{array}$

21.0

\begin{tabular}{|c|c|}
\hline $\begin{array}{l}17.8 \\
3.03 \\
1.97 \\
1.06 \\
0.00\end{array}$ & $\begin{array}{l}13.7 \\
2.95 \\
1.52 \\
1.43 \\
0.00\end{array}$ \\
\hline
\end{tabular}
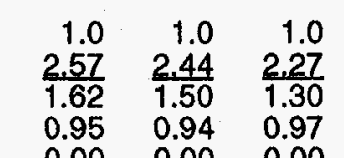

1.0
2.38
1.40
0.98
0.00

1.0
3.28
2.27
1.01

\begin{tabular}{r}
1.0 \\
3.86 \\
\hline 2.87 \\
0.99
\end{tabular}

1.0
$\frac{3.89}{2.90}$
0.99

$\begin{array}{lllll}0.00 & 0.00 & 0.00 & 0.00 & 0.00\end{array}$

$\begin{array}{ll}9.4 & 7.5 \\ .67 & 4.68 \\ .53 & 1.62 \\ 14 & 3.06 \\ 00 & 0.00\end{array}$

\begin{tabular}{lrlllllll}
7.5 & 8.8 & 10.3 & 16.5 & 16.9 & 18.6 & 21.0 & 21.0 & 21.0 \\
4.68 & $\frac{3.75}{1.49}$ & $\frac{3.17}{1.19}$ & $\frac{2.41}{1.19}$ & $\frac{3.15}{1.92}$ & $\frac{3.73}{2.67}$ & $\frac{3.79}{2.85}$ & $\frac{2.53}{1.49}$ & $\frac{1.78}{0.85}$ \\
\hline 1.62 & 1.49 & 1.98 & 1.22 & 1.23 & 1.06 & 0.94 & 1.04 & 0.93 \\
3.06 & 2.26 & 1.98 & 0.00 & 0.00 & 0.00 & 0.00 & 0.00
\end{tabular}

1097

TOTAL MC

$3,091,650$

$3,091,650$

231,260

231,260

648,801

648,801

88,836

88,836

$1,890,090$

$1,890,090$

(1) Volumes used by Michigan Gas Utitities.

(3) Daily contract demand was reduced from 32 MMcf to 21 MMcf, effeclive April 1, 1995. 
VOLUME AND PRICE REPORT

Long-Term Imports

Volumes (MMct/d) \& Prices ( $\$ M M M B T U$ ) of Natural Gas Imported During the Past 12 Months 04/01/96 - 03/31/97

\section{Leng-Term importer}

VERMONT GAS SYSTEMS, INC. (1)

(Various Suppliers

AVG Daily Quantity

Total Price (Highgate Spr. VT)

Commodity Component

Demand Component

Reservation Fee Component

\section{WASHINGTON NATURAL GAS COMPANY}

(Amoco Canada)

AVG Daily Quantity

Total Price (Sumas, Washington)

Commodity Component

Demand Component

Reservation Fee Component

\section{WASHINGTON NATURAL GAS COMPANY} (Mobil Oil Canada)

AVG Daily Quantity

Total Price (Sumas. Washington)

Commodity Component

Demand Component

Reservation Fee Component

WASHINGTON NATURAL GAS COMPANY (Poco Petroleums Ltd.)

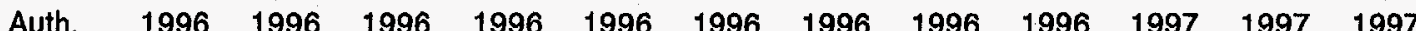

Vols. Apr May Jun. Jul. Aug Sep. Oct. Nov Dec Jan Feb. Mar.

19.0

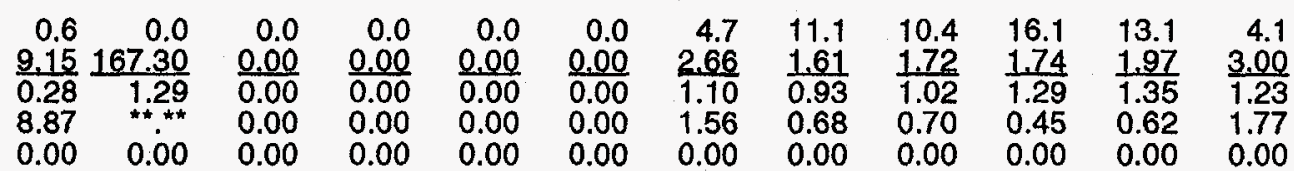

24.0

\begin{tabular}{lll}
24.5 & 25.0 & 24.9 \\
0.98 & 0.97 & 0.93 \\
\hline 0.44 & 0.46 & 0.41 \\
0.54 & 0.51 & 0.52
\end{tabular}

23.7

$\frac{1.00}{0.49}$

$0.00 \quad 0.00$

$\begin{array}{lll}14.8 & 15.0 & 15.0\end{array}$

$\begin{array}{lll}\frac{0.97}{0.33} & \frac{0.95}{0.33} & \frac{0.93}{0.30}\end{array}$

$\begin{array}{lll}0.33 & 0.33 & 0.30 \\ 0.64 & 0.62 & 0.63\end{array}$

$\begin{array}{lll}0.00 & 0.00 & 0.00\end{array}$

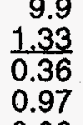

$\frac{1.33}{0.36}$
0.97

0.00

1.84

0.41
1.43

1.43
0.00
AVG Daily Quantity

Total Price (Sumas, Washington)

Commodity Component

Demand Component

Reservation Fee Component

WASHINGTON NATURAL GAS COMPANY (Westcoast Gas Services)

AVG Daily Quantity

Total Price (Sumas, Washington)

Commodity Component

Demand Component

Reservation Fee Component

14.4

\begin{tabular}{lll}
14.6 & 15.0 & 14 \\
0.88 & $\frac{0.86}{0.86}$ & $\underline{0.8}$ \\
\hline 0.46 & 0.47 & 0.43 \\
0.42 & 0.39 & 0.42 \\
0.00 & 0.00 & 0.00
\end{tabular}

$14.9 \quad 15.0$

$\frac{0.90}{0.51} \quad \frac{0.94}{0.55}$

0.39

0.00

0.00

\begin{tabular}{l}
15.0 \\
0.94 \\
\hline 0.54 \\
0.40 \\
0.00
\end{tabular}

9.6

\begin{tabular}{rr}
9.9 & 10.0 \\
0.96 & 0.96 \\
\hline 0.46 & 0.48 \\
0.50 & 0.48 \\
0.00 & 0.00
\end{tabular}

$\begin{array}{ll}0.00 & 0.00\end{array}$ \begin{tabular}{l}
12.0 \\
0.84 \\
\hline 0.43 \\
0.41 \\
0.00
\end{tabular}

$0.00 \quad 0.00$

$\begin{array}{ll}12.0 & 1 \\ 1.10 & 1 \\ 0.63 & 0.10 \\ 0.47 & 0.47 \\ 0.00 & 0.00\end{array}$

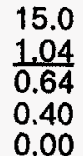

15.0

$\frac{2.10}{1.69}$

15.0

\begin{tabular}{l}
3.48 \\
\hline 3.09
\end{tabular}

0.41

11.5
3.69
0.54

14.5

$\frac{2.31}{1.80}$

15.0

$\frac{0.93}{0.48}$

0.48

0.00

0.39
0.00

0.00

0.00

(1) This import is part of a gas senvices agreement with Gaz Metropolitain Limited Partnership. The demand charge for May was $\$ 166.01$.

$\frac{10}{1.03}$

\begin{tabular}{ll}
10.0 \\
\hline .54
\end{tabular}$\frac{1.13}{0.65}$

$\frac{4.2}{3.7}$

10.0

$\frac{1.02}{0.51}$

0.51

0.00

25.0
1.04
0.52
0.52

25.0
1.13
0.62

0.51

25.0

$\frac{2.20}{1.65}$

0.55

25.0
3.58
3.06
0.52
0.00

16.1
453

$\frac{4.53}{3.59}$

0.94

0.00

25.0
2.41
1.76

0.65

25.0

$\frac{1.09}{0.50}$

0.59

0.00

15.0

14.8

15.0

0.41
0.62

0.62
0.00

$\frac{1.13}{0.49}$

0.64
0.00

$\frac{2.20}{1.56}$

0.64

$\begin{array}{r}15.0 \\ 3.58 \\ \hline\end{array}$

$\frac{3.58}{2.95}$

2.95
0.63

0.63
0.00

11.1
4.43
3.48
0.95

14.2
2.43
1.70

$\frac{2.43}{1.70}$

0.73

0.00

15.0

$\frac{1.01}{0.38}$

0.63
0.00
1097 TOTAL MC

993,212

993,212

$1,975,000$

$1,975,000$

$1,208,187$

$1,208,187$

$1,226,087$

$1,226,087$

870,073

YTD
OTAL MCf

$\begin{array}{rr}9.7 & 10.0 \\ 224 & 3.5\end{array}$

9.6
$\frac{2.42}{1.83}$ 


\section{VOLUME AND PRICE REPORT}

\section{Long-Term Imports}

Volumes (MMcf/d) \& Prices (\$MMBTU) of Natural

Gas Imported During the Past 12 Months 04/01/96 - 03/31/97

Long-Term Importer

WASHINGTON NATURAL GAS COMPANY

(Westcoast Gas Services)

AVG Daily Quantity

Total Price (Eastport. Idaho)

Commodity Component

Demand Component

Reservation Fee Component

WASHINGTON WATER POWER COMPANY (AEC Oil \& Gas Company)

AVG Daily Quantity

Total Price (Eastport, Idaho)

Commodity Component

Demand Component

Reservation Fee Component

WASHINGTON WATER POWER COMPANY

(Amerada Hess Canada Ltd.)

AVG Daily Quantity

Total Price (Eastport. Idaho)

Commodity Componen

Demand Component

Reservation Fee Component

WASHINGTON WATER POWER COMPANY

(PanCanadian Petroleum Limited)

AVG Daily Quantity

Total Price (Eastport, Idaho)

Commodity Component

Demand Component

Reservation Fee Component

WASHINGTON WATER POWER COMPANY

(Westcoast Gas Services)

AVG Daily Quantity

Total Price (Eastport, Idaho)

Commodity Component

Demand Component

Reservation $\mathrm{Fee}$ Component $\begin{array}{lllllllllllll}\text { Auth. } & 1996 & 1996 & 1996 & 1996 & 1996 & 1996 & 1996 & 1996 & 1996 & 1997 & 1997 & 1997\end{array}$

Vols. Apr Mav Jun Jul Aug Sep Oct Nov Dec. Jan. Feb. Mar

95.8

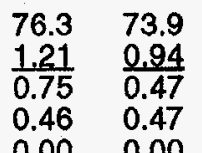

77.4

$\frac{1.15}{0.70}$

71.1
1.22
0.74
0.48

67.7
1.29
0.78
0.51
0.00

76.9
1.22
0.76
0.46

\begin{tabular}{ll}
75.7 & 77.1 \\
1.27 & 1.85 \\
\hline 0.81 & 1.39 \\
0.46 & 0.46 \\
0.00 & 0.00
\end{tabular}

77.1
2.58
2.13
0.45
0.00

77.5

$\frac{2.99}{2.54}$

2.54
0.45

$\frac{2.26}{1.78}$

76.9

0.00

0.00

$0.00 \quad 0.00$

\begin{tabular}{rrr}
0.0 & 0.0 & 0.0 \\
0.00 & 0.00 & 0.00 \\
\hline 0.00 & $\frac{0.00}{0.00}$
\end{tabular}

$\begin{array}{lll}0.00 & 0.00 & 0.00\end{array}$

$\begin{array}{lll}0.00 & 0.00 & 0.00\end{array}$

0.0
0.00
0.00

$\frac{0.00}{0.00}$

0.00
0.00

0.0
0.00
0.00
0.00

$\begin{array}{r}0.0 \\ 0.00 \\ \hline 0.00\end{array}$

0.00
0.00

0.00
0.00

0.0
0.00
0.00
0.00

\begin{tabular}{l}
18.5 \\
1.15 \\
\hline 1.06
\end{tabular}

$\frac{1.15}{1.06}$

0.09

0.00
0.00

18.5
1.15
1.06

$\frac{1.15}{1.06}$

0.09
0.00

$\begin{array}{r}18.5 \\ 1.15 \\ \hline\end{array}$

$\frac{1.15}{1.06}$

0.09

0.09
0.00

$\frac{1.16}{1.06}$

0.10

0.10
0.00

16.8
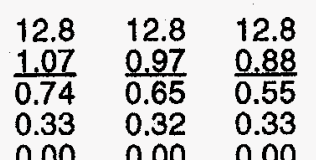

12.8
0.89
0.57
0.32
0.00

12.8
0.95
0.63
0.32
0.00

\begin{tabular}{l}
12.8 \\
0.93 \\
\hline 0.60 \\
0.33 \\
0.00
\end{tabular}

\begin{tabular}{l}
12.8 \\
0.95 \\
\hline 0.63 \\
0.32 \\
0.00
\end{tabular}

\begin{tabular}{l}
13.8 \\
1.14 \\
\hline 0.81 \\
0.33 \\
0.00
\end{tabular}

\begin{tabular}{l}
13.8 \\
1.61 \\
\hline 1.29 \\
0.32 \\
0.00
\end{tabular}

13.8
1.71
1.39
0.32

\begin{tabular}{l}
13.8 \\
2.16 \\
\hline 1.80 \\
0.36 \\
0.00
\end{tabular}

\begin{tabular}{l}
13.9 \\
1.35 \\
\hline 1.03 \\
0.32 \\
0.00
\end{tabular}

19.0

\begin{tabular}{ll}
11.8 & 11.8 \\
1.07 & 0.97 \\
\hline 0.98 & 0.88 \\
0.09 & 0.09
\end{tabular}

11.8
0.89
0.80
0.09

11.8
0.89
0.80
0.09

\begin{tabular}{l}
11.8 \\
0.96 \\
\hline 0.87 \\
0.09
\end{tabular}

11.7
0.94
0.85
0.09

\begin{tabular}{l}
11.7 \\
0.97 \\
\hline 0.88
\end{tabular}

\begin{tabular}{l}
12.7 \\
1.17 \\
\hline 1.08 \\
0.09
\end{tabular}

12.7

$\frac{1.62}{1.53}$

0.09

0.09

1.53
0.09

0.09
0.00

\begin{tabular}{l}
12.7 \\
1.74 \\
\hline 1.65 \\
0.09
\end{tabular}

9.2

$\frac{2.14}{2.05}$

2.05
0.09
0.00

9.2
135

1.35
1.26
0.09

19.1

\begin{tabular}{lllll}
15.3 & 15.3 & 15.3 & 14.7 & 15.3 \\
1.38 & 1.28 & 1.27 & $\frac{1.33}{1.33}$ & $\frac{1.35}{0.82}$ \\
\hline 0.84 & 0.75 & 0.73 & 0.78 & 0 \\
0.54 & 0.53 & 0.54 & 0.55 & 0.53 \\
0.00 & 0.00 & 0.00 & 0.00 & 0.00
\end{tabular}

\begin{tabular}{l}
15.0 \\
1.35 \\
\hline 0.80 \\
0.55 \\
0.00
\end{tabular}

\begin{tabular}{l}
15.2 \\
1.39 \\
\hline 0.85 \\
0.54 \\
0.00
\end{tabular}

\begin{tabular}{l}
14.7 \\
2.04 \\
\hline 1.46 \\
0.58 \\
0.00
\end{tabular}

15.2
2.79
2.25
0.54
0.00

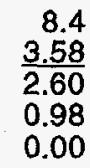

\begin{tabular}{ll}
15.2 & 15.2 \\
2.45 & 1.56 \\
\hline 1.87 & 1.02 \\
0.58 & 0.54 \\
0.00 & 0.00
\end{tabular}
$1,245,590$

$1 Q 97$

TOTAL Ma

$6,947,839$

$6,947,839$

$1,662,880$

$1,662,880$

940,017

940,017

$1,155,818$

$1,155,818$ 
VOLUME AND PRICE REPORT

\section{Long-Term Imports}

Volumes (MMct/d) \& Prices (\$MMBTU) of Natural

Gas Imported During the Past 12 Months 04/01/96 - 03/31/97
Long-Term Importer

$\begin{array}{llll}\text { Auth. } & 1996 & 1996 & 1996 \\ \text { Vols. } & \text { Apr. } & \text { May. Jun. }\end{array}$
1996

Jul.

1996

Aug.

19961996

WESTCOAST GAS SERVICES INC. - CIBOLA CORPORATION

(Westcoast Gas)

AVG Daily Quantity

Total Price (Port of Morgan. MT)

Commodity Component

Demand Component

Reservation Fee Component

1.2

\begin{tabular}{rrr}
1.2 & 1.2 & 1.2 \\
1.93 & 1.75 & 1.78 \\
\hline 1.93 & 1.75 & 1.78 \\
0.00 & 0.00 & 0.00 \\
0.00 & 0.00 & 0.00
\end{tabular}

1.2
$\frac{1.92}{1.92}$
0.00
0.00

1.2
1.92
1.92

$\frac{1.92}{1.92}$

0.00

1.2
1.47
1.47
0.00

0.00

1.2
1.56
1.56
0.00

$\frac{1.2}{2.40}$

2.40
0.00

1.1
2.40
2.40
0.00

$\frac{4.05}{4.05}$

0.00

0.00

$\frac{1.2}{2.70}$

$\frac{1.48}{1.48}$

0.00

WESTCOAST GAS SERVICES INC. - INTERSTATE POWER CO.

(Westcoast Gas)

AVG Daily Quantity

Total Price (Port of Morgan, MT)

Commodity Component

Demand Component

1.0

$\begin{array}{rr}1.0 & 1.1 \\ 2.12 & \frac{1.96}{1.96} \\ 1.88 & 1.73 \\ 0.24 & 0.23 \\ 0.00 & 0.00\end{array}$

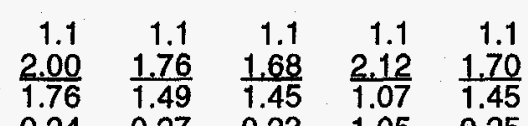

$1.1 \quad 1.1$

Reservation Fee Component
0.24

0.00

0.27

0.23

1.05
0.00

1.1
2.48
2.16
0.32

1.1
3.39
3.10
0.29

1.1
3.82

0.32

0.00

3.53
0.29

1.1
$\frac{2.70}{2.39}$

1.0
1.66

$\frac{1.66}{1.39}$

0.27

0.00

(WESTCOAST GAS

AVG Daily Guant

Total Price (Port of Morgan MT)

3.7

$\begin{array}{rrr}2.4 & 3.7 & 3.7 \\ 2.28 & 2.06 & 2.01 \\ 2.01 & 1.89 & 1.84 \\ 0.27 & 0.17 & 0.17 \\ 0.00 & 0.00 & 0.00\end{array}$

$\begin{array}{rrrrr}3.7 & 3.7 & 3.7 & 3.7 & 3.7 \\ 2.01 & 1.87 & 1.82 & 1.39 & 1.72\end{array}$ $\begin{array}{lll}1.84 & 1.70 & 1.65\end{array}$

Commodity Component

Demand Component

$\begin{array}{lll}0.00 & 0.00 & 0.00\end{array}$

0.17
0.00

0.17
0.00

1.22
0.17

0.17
0.00

$\frac{1.72}{1.56}$
0.16

3.8
2.55
2.37
0.18
0.00

\begin{tabular}{r}
3.8 \\
3.58 \\
\hline 3.41 \\
0.17
\end{tabular}

$\begin{array}{r}3.8 \\ 4.05 \\ \hline 3.88\end{array}$

3.88

WESTCOAST GAS SERVICES INC. - MIDAMERICAN ENERGY (1)

(Westcoast Gas)

AVG Daily Quantity

Total Price (Port of Mergan. MT)

Commodity Component

Demand Component

Reservation Fee Component
6.5

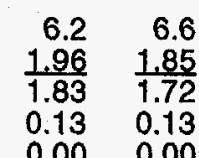

$\begin{array}{rr}6.6 & 6.6 \\ 1.89 & \frac{1.66}{1.76} \\ 1.53\end{array}$

$0.13 \quad 0.13$

\begin{tabular}{r}
6.6 \\
1.61 \\
\hline 1.48 \\
0.13 \\
0.00
\end{tabular}

6.5
1.21
1.08
0.13

$0.00 \quad 0.00$

\begin{tabular}{r}
6.5 \\
1.57 \\
\hline 1.45 \\
0.12 \\
0.00
\end{tabular}

6.7
2.30
2.16
0.14
0.00

6.7
3.27
3.10
0.17

6.7
3.66
3.53
0.13

$\frac{2.53}{2.39}$

0.14
0.00

$\frac{1.53}{1.39}$

0.14

WESTCOAST GAS SERVICES INC. - N'WESTERN PUB. SERV.

(Westcoast Gas)

AVG Daily Quantity

Total Price (Port of Morgan, MT)

0.9

\begin{tabular}{rr}
0.9 & 1.0 \\
2.18 & 2.06 \\
\hline 2.01 & 1.89 \\
0.17 & 0.17 \\
0.00 & 0.00
\end{tabular}

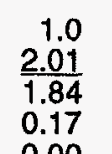

0.17
0.00

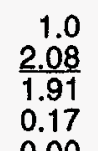

2.08
1.91
0.17
0.00 $\begin{array}{rrr}1.0 & 1.0 & 0.9 \\ 2.04 & \frac{1.61}{1.87} & \frac{3.17}{1.44}\end{array}$

$\begin{array}{lll}1.87 & 1.44 & 1.59 \\ 0.17 & 0.17 & 1.58\end{array}$

$\begin{array}{lll}0.00 & 0.00 & 0.00\end{array}$
0.9
3.91
3.73
0.18
0.00

0.18
0.00 \begin{tabular}{r}
0.9 \\
3.58 \\
\hline 3.41 \\
0.17 \\
0.00
\end{tabular}

\begin{tabular}{r}
0.9 \\
4.05 \\
\hline 3.88 \\
0.17
\end{tabular}

$\frac{2.89}{2.62}$

$\begin{array}{r}1.0 \\ 1.70 \\ \hline 1.53\end{array}$

1.53

0.00
1097

TOTAL MCf

107,461

107,461

95,109

95,109

329,485

329,485

500,227

500,227

76,641

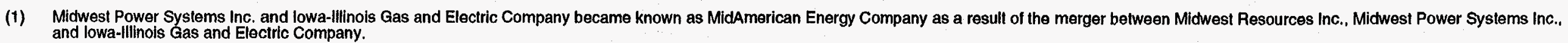




\section{VOLUME AND PRICE REPORT}

\section{Long-Term Imports}

WESTCOAST GAS SERVICES INC. - WISCONSIN GAS CO.

(Westcoast Gas)

AVG Daily Quantity

Total Price (Port of Morgan. MT)

Commodity Component

Demand Component

Reservation Fee Component

$\begin{array}{rrrr}2.3 & 2.4 & 2.4 & 2.4 \\ 2.11 & \frac{1.98}{1.98} & \frac{1.94}{1.80} & \frac{1.79}{1.65} \\ 0.14 & 0.14 & 0.14 & 0.14\end{array}$

\begin{tabular}{rr}
2.5 & 2.4 \\
1.75 & $\frac{1.32}{1.61}$ \\
\hline 1.18 \\
0.14 & 0.14 \\
0.00 & 0.00
\end{tabular}

WISCONSIN EL

(Progas Limited)

Aotal Price (Noyes, Minnesota)

Commodity Componen

Demand Component

Reservation Fee Component

$\begin{array}{llll}0.00 & 0.00 & 0.00 & 0.00\end{array}$

$\begin{array}{lll}.00 & 0.00 & 0.00\end{array}$

1996

1996

WISCONSIN ELECTRIC POWER CO. (GAS OPS) (2)

(TCGS Ltd.)

AVG Daily Quantity

Total Price (Noves, Minnesota)

Commodity Component

Demand Component

Reservation Fee Component

26.5

$\begin{array}{lll}10.7 & 10.6 & 10.6 \\ 2.61 & 2.32 & 2.38 \\ 2.10 & 1.76 & 1.85 \\ 0.48 & 0.53 & 0.50\end{array}$

$\begin{array}{lll}0.48 & 0.53 & 0.50 \\ 0.03 & 0.03 & 0.03\end{array}$

10.7
2.57
2.06
0.48
0.03

\begin{tabular}{lr}
10.7 & 9.9 \\
2.39 & 2.17 \\
\hline 1.88 & 1.40 \\
0.48 & 0.54 \\
0.03 & 0.23
\end{tabular}

10.7
1.92
1.42
0.48
0.02

10.7
2.80
2.26
0.51
0.03

10.7
3.91

$\frac{3.91}{3.37}$

0.49

0.49
0.05

\begin{tabular}{r}
10.7 \\
4.27 \\
\hline 3.72 \\
0.49
\end{tabular}

\begin{tabular}{l}
10.7 \\
\hline 3.07 \\
\hline
\end{tabular}

2.49
0.54

$\begin{array}{ll}0.49 & 0.54 \\ 0.06 & 0.04\end{array}$

10.7

$\frac{1.85}{1.34}$

0.49

\begin{tabular}{lll}
26.5 & 26.3 & 26.4 \\
2.61 & 2.32 & 2.41 \\
\hline 2.07 & 1.79 & 1.86 \\
0.48 & 0.48 & 0.50 \\
0.06 & 0.05 & 0.05
\end{tabular}

25.9
2.59
2.04
0.49
0.06

26.2
2.42
1.88
0.48
0.06

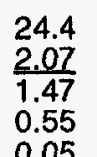

26.2
2.03
1.49
0.50
0.04

26.5
2.81
2.22
0.52
0.07

26.5
3.75
3.17
0.49
0.09

26.5
4.09

26.4
$\frac{3.03}{2.41}$

26.5

$\frac{1.95}{1.42}$

$\begin{array}{lll}0.51 & 0.55 & 0.49\end{array}$

WISCONSIN FUEL \& LIGHT COMPANY

(Progas Limited)

AVG Daily Quantity

Total Price (Neves, Minnesota)

Commodity Component

Demand Component

Reservation Fee Component

3.0

$\begin{array}{rrrr}3.0 & 3.0 & 3.0 & 3.0 \\ 2.52 & 2.19 & 2.28 & 2.45 \\ 1.99 & 1.68 & 1.75 & 1.94 \\ 0.50 & 0.48 & 0.50 & 0.48\end{array}$

3.0
2.29
1.78
0.48
0.03

2.9
1.88
1.35
0.51
0.02

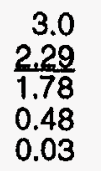

3.0
1.82
1.30
0.50
0.02

3.0
1.86
1.36
0.48

3.0
$\frac{4.06}{3.52}$
0.49

0.07

0.04

\section{WISCONSIN}

AVG Daily Quantity

Total Price (Noyes, Minnesota)

$\begin{array}{lll}0.03 & 0.03 & 0.03\end{array}$

7.4

\begin{tabular}{rrrrrrr}
7.4 & 7.3 & 7.3 & 7.2 & 2.6 & 6.7 & 7.3 \\
2.48 & 2.21 & 2.28 & 2.44 & $\frac{3.20}{1.79}$ & $\frac{2.00}{1.42}$ & $\frac{1.14}{0.64}$ \\
\hline 1.97 & 1.70 & 1.76 & 1.92 & 1.26 & 0.53 & 0.45 \\
0.45 & 0.46 & 0.47 & 0.46 & 1.26 & 0.45 \\
0.06 & 0.05 & 0.05 & 0.06 & 0.15 & 0.05 & 0.05
\end{tabular}

\begin{tabular}{r}
7.4 \\
1.80 \\
\hline 1.28 \\
0.48 \\
0.04
\end{tabular}

7.4
$\frac{1.93}{1.42}$
0.47
0.04

\begin{tabular}{r}
6.7 \\
3.97 \\
\hline 3.32 \\
0.54 \\
0.11
\end{tabular}

4.8
$\frac{3.32}{2.40}$
0.81

7.4

$\frac{1.92}{1.41}$

Reservation Fee Component

$\begin{array}{llll}0.06 & 0.05 & 0.05 & 0.06\end{array}$

0.150 .05

$\begin{array}{ll}0.11 & 0.04\end{array}$

(1) In January 1996. Wisconsin Natural Gas Company became Wisconsin Electric Power Company - Gas Operalions.
(2) January 1996. Wisconsin Natural Gas Company became Wisconsin Electric Power Company - Gas Operations. 


\section{VOLUME AND PRICE REPORT}

\section{Long-Term Imports}

Volumes (MMcf/d) \& Prices (\$MMBTU) of Natural

Gas Imported During the Past 12 Months 04/01/96 - 03/31/97

Long-Term Importer

WISCONSIN GAS COMPANY

(ProGas Limited (I))

AVG Daily Quantity

Total Price (Noves, Minnesota)

Commodity Component

Reservation Fee Component

WISCONSIN GAS COMPANY

(ProGas Limited (II)

AVG Daily Quantity

Total Price (Noyes, Minnesota)

Commodity Component

Demand Component

Reservation Fee Component

WISCONSIN GAS COMPANY

(TCGS Ltd.)

AVG Daily Quantity

Total Price (Noyes. Minnesota)

Commodity Component

Demand Component

Reservation Fee Component

WISCONSIN GAS COMPANY

(TCGS Ltd.)

AVG Daily Quantity

Total Price (Noyes, Minnesota)

Commodity Component

Demand Component

Reservation Fee Component

WISCONSIN POWEA \& LIGHT COMPANY

(ProGas Limited)

AVG Daily Quantity

Total Price (Noyes, Minnesota)

Commodity Componen

Demand Component

Reservation Fee Component

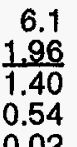

\begin{tabular}{rrr}
1.9 & 1.9 & 1.9 \\
2.58 & 2.29 & 2.39 \\
\hline 2.07 & 1.79 & 1.86 \\
0.45 & 0.45 & 0.47 \\
0.06 & 0.05 & 0.06
\end{tabular}
Apr May Jun. Jul. Aug. Sep. Oct Nov Dec Jan. Feb. Mar.

6.6

\begin{tabular}{rrr}
6.5 & 6.4 & 6.4 \\
2.62 & 2.28 & 2.39 \\
\hline 2.10 & 1.76 & 1.85 \\
0.49 & 0.49 & 0.51 \\
0.03 & 0.03 & 0.03
\end{tabular}

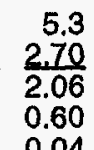

2.40

$\frac{2.40}{1.88}$

0.03

28.5

\begin{tabular}{lll}
28.5 & 28.3 & 28.4 \\
2.61 & 2.27 & 2.38 \\
\hline 2.10 & 1.76 & 1.85 \\
0.48 & 0.48 & 0.50
\end{tabular}

$\begin{array}{lll}0.03 & 0.03 & 0.50 \\ 0.03\end{array}$

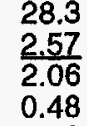

$\frac{2.57}{2.06}$

0.48
0.03

0.49

$\begin{array}{r}6.4 \\ 1.94 \\ \hline\end{array}$

$\frac{1.94}{1.44}$

$\begin{array}{ll}0.48 \\ 0.02 & 0.02\end{array}$

6.5
2.78
2.26
0.49

0.49
0.03

6.5
3.91
3.37
0.49
0.05

6.5
$\frac{4.28}{3.72}$

0.50

6.5
$\frac{3.08}{2.49}$
0.55

0.55

6.5
1.85
1.34

$\frac{1.85}{1.34}$

0.49

0.02

89.4

\begin{tabular}{lll}
89.1 & 88.6 & 88.8 \\
2.58 & 2.30 & 2.39 \\
\hline 2.07 & 1.79 & 1.86 \\
0.45 & 0.46 & 0.47 \\
0.06 & 0.05 & 0.06
\end{tabular}

87.9

$\frac{2.56}{2.04}$

2.04
0.46

0.46
0.06

28.3
2.39
1.88
0.48

26.4

29.8

28.5
2.78
2.26

$\frac{1.96}{1.40}$
0.54

$\frac{1.94}{1.44}$

$\begin{array}{lll}0.48 & 0.54 & 0.48\end{array}$

0.49

$\begin{array}{r}28.5 \\ 3.89 \\ \hline 3.37\end{array}$

$\frac{3.89}{3.37}$

3.37
0.47

$\begin{array}{lll}22.1 & 30.5 & 28.7\end{array}$

$\frac{4.43}{3.72}$

$\frac{2.80}{2.26}$

3.72
0.64

0.51

$\frac{1.84}{1.34}$

0.48

0.02

1.9

1.9
2.55
2.04
0.45

1.9
2.38
1.88

$\begin{array}{ll}0.45 & 0.44 \\ 0.06 & 0.06\end{array}$

88.3
2.38

$\frac{2.38}{1.88}$

0.45
0.05

82.4

$\frac{2.04}{1.47}$

68.2

70.4

70.4

0.52
0.05

1.49
0.47
0.04

$\frac{2.80}{2.22}$

0.51
0.07

$\frac{3.74}{3.17}$

89.1
4.08
3.48

57.1
3.35

89.2

$\begin{array}{lll}\frac{4.08}{3.48} & \frac{3.35}{2.41} & \frac{1.95}{1.42} \\ 0.50 & 0.83 & 0.49\end{array}$

\begin{tabular}{ll}
$0.11 \quad 0.04$ \\
\hline
\end{tabular}

$\begin{array}{lll}0.06 & 0.05 & 0.06\end{array}$

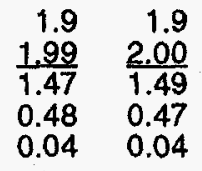

1.9
2.80
2.22
0.51
0.07

1.9
3.74
3.17
0.48

1.9
4.08
3.48
0.50

1.9
3.01
2.41
0.5

1.9

$\frac{2.31}{1.42}$

1.42
0.47

0.42

3.4

\begin{tabular}{rrr}
3.4 & 3.4 & 3.4 \\
2.63 & 2.27 & 2.38 \\
\hline 2.10 & 1.76 & 1.85 \\
0.53 & 0.51 & 0.53 \\
0.00 & 0.00 & 0.00
\end{tabular}

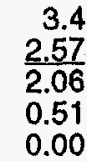

\begin{tabular}{r}
3.4 \\
2.57 \\
\hline 2.06 \\
0.51 \\
0.00
\end{tabular}

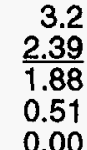

\begin{tabular}{r}
3.2 \\
2.39 \\
\hline 1.88 \\
0.51 \\
0.00
\end{tabular}

$\begin{array}{r}3.3 \\ 1.94 \\ \hline\end{array}$

$\frac{1.94}{1.40}$

$0.54 \quad 0.51$

$0.00 \quad 0.00$ $\begin{array}{r}3.4 \\ 2.80 \\ \hline\end{array}$

2.26

0.54
0.00
3.4

$\frac{3.91}{3.37}$

0.54
0.00

\begin{tabular}{r}
3.4 \\
4.27 \\
\hline 3.72 \\
0.55
\end{tabular}

3.4
$\frac{3.07}{2.49}$ $\begin{array}{ll}0.00 & 0.00\end{array}$
1097

IOTAL MCf

587,057

587,057

$2,431,403$

$2,431,403$

$7,123,671$

$7,123,671$

171,088

171,088

304,889

304,889 
可

$\frac{0}{N}$

\$ㅇำ

ริ

ఫิ

\%윰 정ํㅠ융

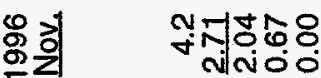

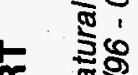

뜽 홍

용ㅎㅇ

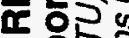

u 을

แั

:

으ㅁㅗㅗㅇ

든

응

2 엉뭉

山 둥요

옥

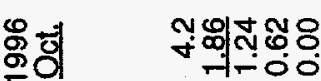

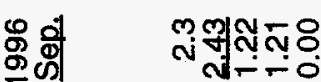

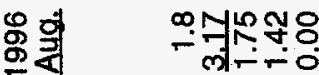

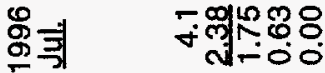

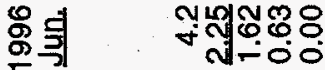

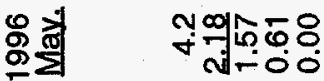

क⿺ ํํㅇ

동

इ

영

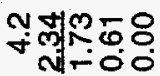

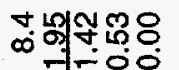

テ내유용

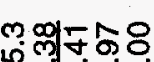

एलंखं०

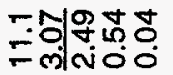

๓ 윕

โNNํํำ

Fौल0

कNㅟㅇㅇㅇㅇㅇㅇ

뎨용ㅇㅇ

Fलीं००

m ๙ लํำ

임유요

न लों००

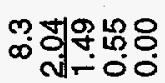

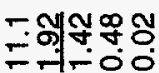

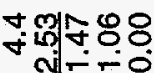

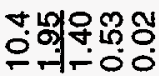

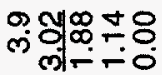

ㅍํำ

ํㅝํ용유

๓ ํㅐ용

m mis

m다옹용

๙ बำ

팡으뭉용

- Nivio

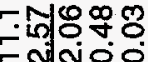

=

־상요

กํํำ

우의은용

영ํํ - 8

Nivió

역궘ㅇㅇㅇ

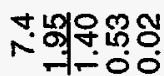

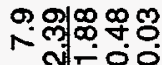

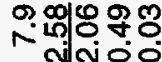

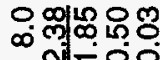

Ninoo

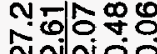

芒

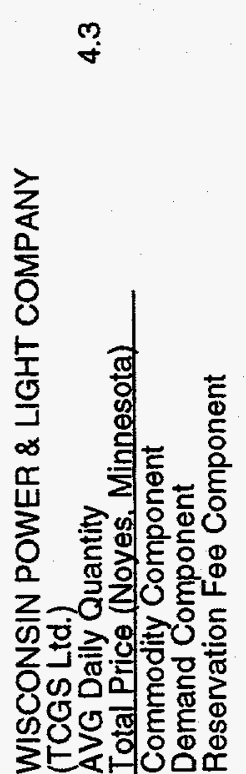

$\stackrel{+}{\infty}$
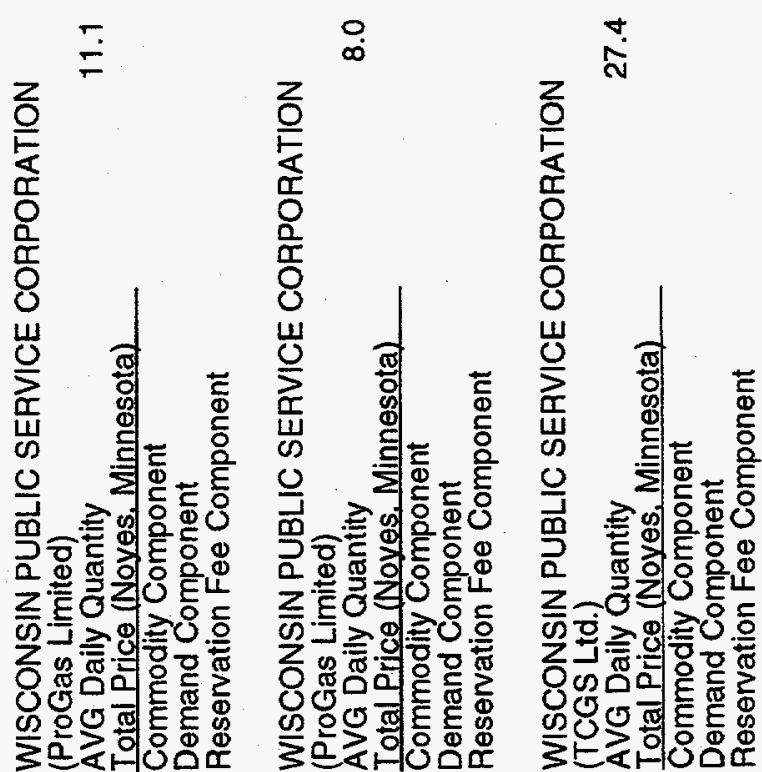
Leng-Term Importer

YANKEE GAS SERVICES $C O$.

(Alberta NE Gas (AEC Oil \& Gas Company)) AVG Daily Quantity

Total Price (Waddington. NY)

Demand Component

Reservation Fee Component

YANKEE GAS SERVICES CO.

(Alberta NE Gas (TCGS Ltd. 1))

AVG Daily Quantity

Total Price (Waddington, NY)

Commodity Component

Demand Component

Reservation Fee Component

YANKEE GAS SERVICES CO.

(Alberta NE Gas (TCGS Ltd. 2))

AVG Daily Quantity

Total Price (Waddington, NY)

Commodity Component

Demand Component

Reservation Fee Component

YANKEE GAS SERVICES CO

(Alberta Northeast Gas (ProGas))

AVG Daily Quantity

Total Price (Waddington, NY)

Commodity Component

Demand Component

Reservation Fee Component

YANKEE GAS SERVICES CO.

(Alberta Northeast Gas (Producers Marketing))

AVG Daily Quantity

Total Price (Waddington. NY)

Commodity Component

Demand Component

Reservation Fee Component

\section{VOLUME AND PRICE REPORT}

\section{Long-Term Imports}

Volumes (MMcf/d) \& Prices ( $\$$ /MMBTU) of Natural

Gas Imported During the Past 12 Months 04/01/96 - 03/31/97

$\begin{array}{llllllll}\text { Auth. } & 1996 & 1996 & 1996 & 1996 & 1996 & 1996 & 1996\end{array}$

3.9

$\begin{array}{rrrrrrrrrrrrr}3.9 & 3.9 & 3.9 & 3.9 & 3.9 & 3.9 & 3.9 & 3.9 & 3.9 & 3.9 & 3.9 & 3.9 \\ 2.74 & 2.67 & 2.60 & 2.56 & 2.44 & 2.27 & 2.38 & 3.27 & \frac{3.86}{3.88} & \frac{3.88}{3.37} & \frac{2.63}{2.93} \\ 1.83 & 1.74 & 1.67 & 1.63 & 1.52 & 1.34 & 1.43 & 2.32 & 2.79 & 2.94 & 2.41 & 1.65 \\ 0.91 & 0.93 & 0.93 & 0.93 & 0.92 & 0.93 & 0.95 & 0.95 & 1.07 & 0.94 & 0.96 & 0.98 \\ 0.00 & 0.00 & 0.00 & 0.00 & 0.00 & 0.00 & 0.00 & 0.00 & 0.00 & 0.00 & 0.00 & 0.00\end{array}$

28.7

\begin{tabular}{lll}
28.7 & 28.7 & 28.5 \\
2.74 & 2.67 & 2.60 \\
\hline 1.80 & 1.72 & 1.64 \\
0.94 & 0.95 & 0.96
\end{tabular}

$\begin{array}{lll}0.00 & 0.00 & 0.00\end{array}$

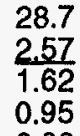

28.7

$\frac{28.7}{1.27}$

1.50

0.94

0.97

28.7

$\frac{2.38}{1.40}$

1.40
0.98

28.7
3.28

$\frac{3.28}{2.27}$

1.01

$\begin{array}{r}28.7 \\ 3.86 \\ \hline 2.87\end{array}$

$\frac{3.86}{2.87}$

2.87
0.99

0.00 .

28.7

$\frac{3.89}{2.90}$

0.99

3.37

0.98
0.00

11.3

$\begin{array}{lrr}11.3 & 9.9 & 11 \\ 2.74 & 2.67 & 2.6 \\ 1.80 & 1.72 & 1.64 \\ 0.94 & 0.95 & 0.96 \\ 0.00 & 0.00 & 0.00\end{array}$

11.3

11.3

11.3

11.3

11.3

11.3

$\frac{2.60}{1.64} \quad \frac{2.57}{1.62}$

1.62
0.95
0.00

$\frac{2.44}{1.50}$

0.94
0.00

1.30

0.97

1.40

0.98
0.00

$\frac{3.28}{2.27}$

1.01

$\frac{3.86}{2.87}$

$\begin{array}{r}11.3 \\ 3.89 \\ \hline\end{array}$

$2.87 \quad 2.90$

\begin{tabular}{l}
11.3 \\
3.37 \\
\hline
\end{tabular}

$\frac{3.37}{2.39}$

0.98

$\frac{2.62}{1.66}$

0.96

0.00

9.9

$\begin{array}{rrr}9.9 & 9.9 & 9.9 \\ 2.82 & 2.76 & 2 \\ 1.82 & 1.77 & 1 \\ 1.00 & 0.99 & 1.00 \\ 0.00 & 0.00 & 0.00\end{array}$

9.9
2.69
1.69
1.00
0.00

9.9
265

$9.9 \quad 9.9$

9.9

9.9$$
1
$$

$\frac{2.65}{1.66} \cdot \frac{2.52}{1.53}$

0.99

1.53
0.99

$\frac{2.34}{1.35}$

$\frac{2.47}{1.46}$

1.01
0.00

2.14
1.02

1.02
0.00

9.9
3.71
2.71
1.00
0.00

0.00

\begin{tabular}{r}
9.9 \\
4.00 \\
\hline 2.99 \\
1.01 \\
0.00
\end{tabular}

9.9
3.25
2.25
1.00

9.9

$\frac{2.53}{1.54}$

1.54

0.99

5.2

\begin{tabular}{|c|c|}
\hline $\begin{array}{r}5.2 \\
2.74 \\
1.80 \\
0.94 \\
0.00\end{array}$ & $\begin{array}{r}5.2 \\
2.67 \\
1.72 \\
0.95 \\
0.00\end{array}$ \\
\hline
\end{tabular}

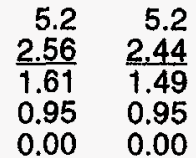

5.2
2.44
1.49
0.95
0.00

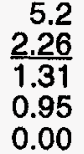

\begin{tabular}{r}
5.2 \\
2.38 \\
\hline 1.42 \\
0.96 \\
0.00
\end{tabular}

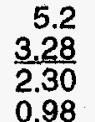

5.2
$\frac{3.28}{2.30}$
0.98
0.00

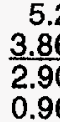

$\begin{array}{r}5.2 \\ 3.86 \\ \hline 2.90\end{array}$

$\frac{3.86}{2.90}$
0.96

\begin{tabular}{r}
5.2 \\
3.89 \\
\hline 2.92 \\
0.97 \\
0.00
\end{tabular}

5.2
3.37
2.41
0.96
0.00

5.2
2.62
1.67
0.95
0.00
1097

TOTAL MCf

YTD

TOTAL Mcf

353,520

353,520

$2,583,000$

$2,583,000$

891,000

891,000

,

469,980

469,980 
Page - 56

Altachment B-1

\section{VOLUME AND PRICE REPORT}

\section{Long-Term Imports}

Volumes (MMct/d) \& Prices (\$MMBTU) of Natural

Gas Imported During the Past 12 Months 04/01/96 - 03/31/97

Long-Term Importer

$1 \mathrm{Q97}$
IOTAL MCF

$372,081,005$
YTD

TOTAL MCI

Total Mef 
Page - 57

\section{VOLUME AND PRICE REPORT}

\section{Long Term Exports}

Volumes (MMcf/d) \& Prices (\$/MMBTU) of Natural Gas Exported During the Past 12 Months 04/01/96 - 03/31/97

\section{Long-Term Exporter}

MARATHON OIL COMPANY *

(Tokyo Electric Co./Tokyo Gas Co.)

AVG Daily Quantity

Total Price (Kenai. Alaska)

Commodity Component

Demand Component

Reservation Fee Component

Auth.

Auth.
Vols.

1996
Apr.

50.4

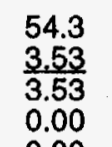

34.53
$\begin{aligned} & 3.53 \\ & 0.00 \\ & 0.00\end{aligned}$

\begin{tabular}{ll}
39.4 & 60.6 \\
3.57 & 3.61 \\
\hline 3.57 & 3.61 \\
0.00 & 0.00 \\
0.00 & 0.00
\end{tabular}

60.6
3.61
3.61
0.00

76.3
3.62
3.62
0.00
0.00

\begin{tabular}{ll}
6.3 & 50.4 \\
.62 & 3.63 \\
\hline .62 & 3.63 \\
.00 & 0.00 \\
.00 & 0.00
\end{tabular}

\begin{tabular}{l}
55.8 \\
3.69 \\
\hline 3.69 \\
0.00 \\
0.00
\end{tabular}

55.1
3.80
3.80
0.00

3.97

$\begin{array}{lllll}3.6 & 54.0 & 53.8 & 62.2 & 54.6 \\ 3.97 & \frac{4.10}{4.97} & \frac{4.21}{4.21} & \frac{3.97}{3.97} & \frac{3.97}{3.97} \\ .00 & 0.00 & 0.00 & 0.00 & 0.00 \\ 0.00 & 0.00 & 0.00 & 0.00 & 0.00\end{array}$

PHILIIPS ALASKA NATURAL GAS CORPORATION

(Tokyo Electric Co./Tokyo Gas Co.)

AVG Daily Quantity

Total Price (Kenai, Alaska)

Commodity Componen

Demand Component

Reservation Fee Component

$\begin{array}{rrrrrrrrrrrr}134.2 & 81.6 & 127.8 & 167.2 & 132.3 & 132.9 & 125.1 & 135.4 & 128.8 & 126.9 & 137.6 & 125.1 \\ \frac{3.53}{3.53} & \frac{3.57}{3.57} & \frac{3.61}{3.61} & \frac{3.62}{3.62} & \frac{3.63}{3.63} & \frac{3.69}{3.69} & \frac{3.80}{3.80} & \frac{3.97}{3.97} & \frac{4.10}{4.10} & \frac{4.21}{4.21} & \frac{3.97}{3.97} & \frac{3.97}{3.97} \\ 0.00 & 0.00 & 0.00 & 0.00 & 0.00 & 0.00 & 0.00 & 0.00 & 0.00 & 0.00 & 0.00 & 0.00 \\ 0.00 & 0.00 & 0.00 & 0.00 & 0.00 & 0.00 & 0.00 & 0.00 & 0.00 & 0.00 & 0.00 & 0.00\end{array}$

Total Mof

136.4
Attachment B-2

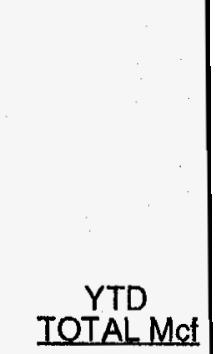

$5,105,631 \quad 5,105,631$

$11,666,063$

$11,666,063$

$\underline{16,771,694}$

16.771 .694 


\section{SHORT-TERM BLANKET IMPORTS}

Year \&

Month

Active

Importers

\author{
Est. Volumes \\ (MMCF)
}

Weighted Avg.

Price (\$/MMBTU)

1995

$\begin{array}{lr}\text { January } & 98 \\ \text { February } & 102 \\ \text { March } & 97 \\ \text { April } & 89 \\ \text { May } & 88 \\ \text { June } & 88 \\ \text { July } & 85 \\ \text { August } & 89 \\ \text { September } & 92 \\ \text { October } & 91 \\ \text { November } & 92 \\ \text { December } & 93\end{array}$

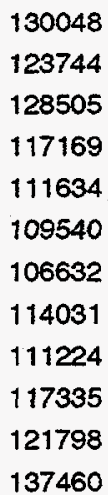

137460
1.28

1.15

1.10

1.03

1.12

1.13

1.06

0.99

1.07

1.14

1.29

1.52

1996

January

February

March

April

May

June

July

August

September

October

November

December

1997

January

February

March
96

97

94
138134

116105

118577

115666

130878

116529

116820

115353

121883

119023

123337

139808

144203

123044

132301
1.89

1.71

1.61

1.47

1.34

1.30

1.43

1.38

1.27

1.33

1.94

2.76

2.98

2.18

1.43 


\section{SHORT-TERM IMPORTERS}

Estimated Volumes (MMCF)

AEC WEST LTD.

AIG TRADING CORPORATION

ALENCO GAS SERVICES INC.

ALUMINUM COMPANY OF AMERICA

AMERADA HESS CORPORATION

AMOCO CANADA MARKETING CORPORATION

AMOCO ENERGY TRADING CORPORATION

ANADARKO TRADING COMPANY

AQUILA ENERGY MARKETING CORPORATION

ARCO PRODUCTS COMPANY

BAY STATE GAS COMPANY

BOUNDARY GAS, INC.

CABOT OIL \& GAS MARKETING CORPORATION

CANSTATES GAS MARKETING (U.S.) LTD.

CANWEST GAS SUPPLY U.S.A., INC.

CASCADE NATURAL GAS CORPORATION

CHANNEL GAS MARKETING COMPANY

CHEVRON U.S.A. INC.

CIBOLA CORPORATION

CNG ENERGY SERVICES CORPORATION

COASTAL GAS MARKETING COMPANY

COENERGY TRADING COMPANY

CONOCOINC.

CONSUMERS ENERGY COMPANY

CONWEST EXPLORATION COMPANY LIMITED

CORAL ENERGY RESOURCES, L.P.

CORPUS CHRISTI GAS MARKETING, L.P.

COWEST ENERGY

CRESTAR ENERGY MARKETING CORPORATION

DARTMOUTH POWER ASSOCIATES L.P.

DEK ENERGY COMPANY

DIRECT ENERGY MARKETING LIMITED

DISTRIGAS CORPORATION (1)

EASTERN ENERGY MARKETING, INC.

EL PASO ENERGY MARKETING COMPANY

ENRON CAPITAL \& TRADE RESOURCES CORP.

ENSERCH GAS COMPANY

EQUITABLE RESOURCES MARKETING COMPANY

GAZ METROPOLITAIN AND COMPANY, L.P.

GREAT FALLS GAS COMPANY

GULF ENERGY MARKETING COMPANY

HOWARD ENERGY COMPANY, INC.

HUSKY GAS MARKETING, INC.

IGI RESOURCES INC.

INDECK ENERGY SERVICES, INC.

INLAND PACIFIC ENERGY SERVICES CORP.

INLAND PACIFIC ENERGY SERVICES LTD.

\begin{tabular}{|c|c|c|c|c|}
\hline $\begin{array}{c}1996 \\
\text { Jan.-Mar. }\end{array}$ & $\begin{array}{c}1996 \\
\text { Apr-Jun. }\end{array}$ & $\begin{array}{c}1996 \\
\text { Jul.-Sep. }\end{array}$ & $\begin{array}{c}1996 \\
\text { Oct.-Dec. }\end{array}$ & $\begin{array}{c}1997 \\
\text { Jan.-Mar. }\end{array}$ \\
\hline 0 & 0 & 2120 & 1909 & 1319 \\
\hline 6590 & 3371 & 3511 & 1545 & 1890 \\
\hline 1296 & 2230 & 2654 & 3056 & 3351 \\
\hline 0 & 262 & 396 & 460 & 439 \\
\hline 1810 & 1818 & 1836 & 619 & 0 \\
\hline 6165 & 8463 & 9512 & 8023 & 8385 \\
\hline 1690 & 630 & 247 & 112 & 578 \\
\hline 138 & 1216 & 289 & 138 & 1054 \\
\hline 0 & 0 & 87 & 608 & 714 \\
\hline 689 & 1025 & 1277 & 1473 & 984 \\
\hline 17 & 0 & 0 & 0 & 0 \\
\hline 0 & 0 & 0 & 0 & 96 \\
\hline 468 & 485 & 848 & 606 & 466 \\
\hline 3533 & 4811 & 244 & 0 & 0 \\
\hline 15389 & 14717 & 14784 & 16051 & 15828 \\
\hline 2263 & 1139 & 695 & 2431 & 3543 \\
\hline 0 & 0 & 24 & 0 & 0 \\
\hline 7357 & 8404 & 4507 & 118 & 1260 \\
\hline 765 & 608 & 0 & 265 & 2259 \\
\hline 4469 & 541 & 0 & 2396 & 3068 \\
\hline 10429 & 16314 & 17948 & 16009 & 17283 \\
\hline 5785 & 3466 & 4446 & 4880 & 5580 \\
\hline 0 & 0 & 0 & 0 & 5 \\
\hline 1365 & 1136 & 1035 & 1264 & 1350 \\
\hline 3168 & 2537 & 0 & 0 & 0 \\
\hline 0 & 0 & 0 & 1021 & 1664 \\
\hline 542 & 304 & 0 & 45 & 154 \\
\hline 117 & 0 & 0 & 385 & 34 \\
\hline 577 & 745 & 451 & 180 & 0 \\
\hline 224 & 153 & 101 & 891 & 451 \\
\hline 740 & 1052 & 1034 & 590 & 350 \\
\hline 81 & 0 & 0 & 0 & 0 \\
\hline 0 & 0 & 1950 & 2885 & 2366 \\
\hline 1759 & 934 & 242 & 958 & 2455 \\
\hline 182 & 997 & 701 & 695 & 247 \\
\hline 3939 & 9272 & 11938 & 10971 & 10303 \\
\hline 3836 & 2848 & 2651 & 4062 & 3060 \\
\hline 1321 & 4680 & 3728 & 4772 & 5369 \\
\hline 26 & 0 & 0 & 0 & 0 \\
\hline 1005 & 282 & 206 & 676 & 1055 \\
\hline 233 & 70 & 0 & 0 & 0 \\
\hline 404 & 7 & 371 & 0 & 293 \\
\hline 6887 & 6641 & 6655 & 8413 & 9781 \\
\hline 12332 & 10176 & 9421 & 10355 & 10660 \\
\hline 497 & 217 & 219 & 216 & 207 \\
\hline 350 & 324 & 331 & 286 & 250 \\
\hline 1840 & 1709 & 2371 & 3408 & 2845 \\
\hline
\end{tabular}

(1) This figure represents sales for resale rather than imports. Actual imports are as follows: 3 rd Qtr $96(2,524) ; 4$ th $Q$ tr 96 (2,424); 1 st Qtr 97 (2,417). 


\section{SHORT-TERM IMPORTERS Estimated Volumes (MMCF)}

INTALCO ALUMINUM CORPORATION INTERENERGY CORPORATION IROQUOIS ENERGY MANAGEMENT, INC.

JONAN GAS MARKETING

KCS ENERGY MARKETING, INC.

KIMBALL ENERGY CORPORATION

KOCH ENERGY TRADING, INC.

KOCH GAS SERVICES COMPANY

LG\&E NATURAL MARKETING INC.

MASSPOWER

MIDCON GAS SERVICES CORPORATION

MOBIL NATURAL GAS INC.

MURPHY GAS GATHERING

N.Y. STATE ELECTRIC \& GAS CORPORATION

NATIONAL FUEL GAS DISTRIBUTION

NATIONAL FUEL RESOURCES, INC.

NATIONAL STEEL CORPORATION

NATURAL GAS CLEARINGHOUSE

NEW ENGLAND POWER COMPANY

NMU GAS PURCHASING INC.

NORAM ENERGY SERVICES, INC.

NORSTAR ENERGY, L.P.

NORTH AMERICAN ENERGY, INC.

NORTH AMERICAN RESOURCES COMPANY

NORTH CANADIAN MARKETING CORPORATION

NORTHERN STATES POWER COMPANY (WISCONSIN)

NORTHERN UTILITIES, INC.

NUMAC ENERGY (U.S.) INC.

OCEAN STATE POWER

OCEAN STATE POWER II

ONYX GAS MARKETING COMPANY, L.C.

PAN-ALBERTA GAS (U.S.), INC.

PANCANADIAN PETROLEUM COMPANY

PANENERGY GAS SERVICES, INC.

PANENERGY LNG SALES, INC. (1)

PANENERGY TRADING AND MARKET SERVICES L.L.

PARAMOUNT RESOURCES U.S., INC.

PAWTUCKET POWER ASSOCIATES L.P.

PETRO-CANADA HYDROCARBONS, INC.

PG\&E GAS SUPPLY BUSINESS UNIT

PHIBRO INC.

POCO MARKETING LTD.

POCO PETROLEUM, INC.

PORTLAND GENERAL ELECTRIC COMPANY

PROGAS U.S.A., INC.

PUGET SOUND ENERGY (2)

QUESTAR ENERGY TRADING COMPANY
1996

Jan.-Mar.

1996
Apr.-Jun.

155

991

1361

0

1699
407

0

1391

135

0
288

10020

296

0
1826

1251

383

10

520

1251

376

219

0

20

10529

186

312

1845

18

18

125

6081

13362

13327

618

0

1564

129

7137

49584

4648

0

10214

1538

9076

908

348
1996

Jul.-Sep

142

1538

929

201

1339

353

0

1118

16

81

382

3561

299

5

142

116

2289

120

35

995

67

218

265

0

13004

12570

0

1812

0

0

75

5388

14593

12297

468

0

296

271

7046

47219

2985

10217

0

107

6057

907

658

1831

0

0

10

5720

14690

18647

183

0

94

168

7487

46570

1334

11651

0

4628

5160

778

152
1996
Oct.-Dec.

152

1518

987

263

1602

312

0

1194

134

0

37

0

299

5

719

2264

1554

282

516

422

67

248

470

23

13202

0

0

14863

220

477

23690

0

28

5244

52366

1302

10239

0

4375

5061

913

346
1997

Jan.-Mar.

140

1030

968

12

1475

0

51

1068

1988

247

0

0

293

205

1331

1730

921

1052

230

369

195

223

388

92

12786

0

0

2691

0

441

7686

14667

(1) These figures represent sales for resale rather than imports. Actual imports are as follows: 1st Qtr 96 (0); 2nd Otr 96 (1,960); 3rd Qtr 96 (0); 4 th Qtr 96 (655); 1st Qtr 97 (762). 


\section{SHORT-TERM IMPORTERS \\ Estimated Volumes (MMCF)}

RENAISSANCE ENERGY (U.S.), INC.

RIGEL OIL \& GAS LTD.

ROCHESTER GAS \& ELECTRIC CORPORATION

SALMON RESOURCES LTD.

SAN DIEGO GAS \& ELECTRIC

SELKIRK COGEN PARTNERS, L.P.

SEMCO ENERGY SERVICES

SIERRA PACIFIC POWER COMPANY

SONAT MARKETING COMPANY

SOUTHERN CALIFORNIA EDISON COMPANY

ST. CLAIR PIPELINES LTD.

ST. LAWRENCE GAS COMPANY, INC.

STAMPGAS (U.S.) INC.

SUMAS COGENERATION COMPANY, LP

SUNCOR INC.

TALISMAN MARKETING (U.S.)

TARPON GAS MARKETING LTD.

TENASKA MARKETING VENTURES

TENNECO GAS MARKETING COMPANY

TEXACO NATURAL GAS INC.

TEXAS-OHIO GAS, INC.

THE BROOKLYN UNION GAS COMPANY

THE MONTANA POWER COMPANY

TRANSCANADA GAS SERVICES INC.

TRANSCO ENERGY MARKETING COMPANY

UNION PACIFIC FUELS, INC.

VALERO GAS MARKETING, L.P.

VASTAR GAS MARKETING

VERMONT GAS SYSTEMS, INC.

WASCANA ENERGY MARKETING (U.S.) INC.

WASHINGTON ENERGY GAS MARKETING COMPANY

WASHINGTON WATER POWER COMPANY

WESTCOAST GAS SERVICES (AMERICA) INC.

WESTCOAST GAS SERVICES (U.S.A.), INC.

WESTCOAST GAS SERVICES INC.

WESTERN GAS RESOURCES, INC.

WILLIAMS ENERGY SERVICES COMPANY

Totals

\begin{tabular}{|c|c|c|c|c|}
\hline $\begin{array}{c}1996 \\
\text { Jan.-Mar. }\end{array}$ & $\begin{array}{c}1996 \\
\text { Apr.-Jun. }\end{array}$ & $\begin{array}{c}1996 \\
\text { Jul.Sep. }\end{array}$ & $\begin{array}{c}1996 \\
\text { Oct.-Dec. }\end{array}$ & $\begin{array}{l}1997 \\
\text { Jan.-Mar. }\end{array}$ \\
\hline 13352 & 9185 & 8786 & 8804 & 9221 \\
\hline 359 & 360 & 357 & 118 & 0 \\
\hline 0 & 0 & 0 & 900 & 2715 \\
\hline 3187 & 3335 & 3007 & 3081 & 3007 \\
\hline 710 & 1657 & 1026 & 2783 & 2838 \\
\hline 599 & 475 & 104 & 1002 & 432 \\
\hline 10 & 0 & 0 & 378 & 145 \\
\hline 5797 & 3101 & 3134 & 4938 & 5754 \\
\hline 346 & 126 & 1605 & 44 & 87 \\
\hline 2005 & 3352 & 4635 & 3227 & 3486 \\
\hline 0 & 0 & 0 & 0 & 235 \\
\hline 0 & 0 & 95 & 95 & 494 \\
\hline 1 & 782 & 781 & 586 & 785 \\
\hline 16 & 0 & 0 & 48 & 44 \\
\hline 9166 & 8291 & 7739 & 8263 & 8468 \\
\hline 12 & 0 & 0 & 0 & 0 \\
\hline 4 & 0 & 0 & 0 & 0 \\
\hline 1553 & 1655 & 236 & 1725 & 3603 \\
\hline 400 & 977 & 159 & 456 & 0 \\
\hline 49 & 167 & 0 & 0 & 845 \\
\hline 261 & 196 & 72 & 51 & 0 \\
\hline 41 & 0 & 0 & 0 & 51 \\
\hline 966 & 532 & 0 & 1498 & 1767 \\
\hline 50881 & 50239 & 47021 & 48077 & 52233 \\
\hline 165 & 35 & 422 & 407 & 119 \\
\hline 857 & 1018 & 973 & 151 & 18 \\
\hline 149 & 110 & 0 & 0 & 61 \\
\hline 0 & 0 & 0 & 315 & 464 \\
\hline 193 & 33 & 0 & 0 & 267 \\
\hline 5248 & 5685 & $\mathbf{5 5 5 0}$ & 7725 & 9088 \\
\hline 231 & 0 & 0 & 0 & 0 \\
\hline 2350 & 1166 & 3040 & 4194 & 3358 \\
\hline 4965 & 4686 & 4844 & 5290 & 5983 \\
\hline 0 & 158 & 0 & 1017 & 1428 \\
\hline 4356 & 7029 & 4451 & 6716 & 5672 \\
\hline 0 & 315 & 0 & 103 & 0 \\
\hline 340 & 535 & 869 & 869 & 0 \\
\hline 372816 & $\underline{363073}$ & $\underline{354056}$ & 382168 & 399549 \\
\hline
\end{tabular}


PURCHASERS OF SHORT-TERM GAS

Estimated Volumes Imported (MMCF)

ADP

AGF Direct Gas

AIG Trading Corporation

ANR Pipeline Company

Alcan

Alliance

Alliance Energy Services

Allied - Signal

Altresco Pittsfield, L.P.

Aluminum Company of America

AmGas inc.

Amalgamated Sugar Company

Amerada Hess

American Fine Foods

American National Power Co.

Amoco Trading

Anadarko Trading Company

Aquila Energy Marketing

Arcadian Corporation

Arco Products Company

Armstrong Wordd Industries, Inc.

Asarco Inc.

Ash Grove Cement

Ash Grove Cement West

Associated Gas Services, Inc.

Ault Naval Base

Aurora Natural Gas

Austin

BC Gas inc.

BNG, Inc.

Ball Corporation

Baltimore Gas \& Electric

Baltimore Natural Gas

Barret Fuels

Barretts Minerals

Basic American Foods

Bay State Gas Company

Beacon Management

Beldon and Blake

Berkshire Gas Company

Big West

Birmingham Steel

Boeing Company

Boise Cascade Corporation

Borden

Boston Edison Company

Boston Gas Company

Brooklyn Union Gas

Buffalo Paperboard Corporation

Bullseye Glass
1996

Jan.-Mar.

0

30
6128

372

0

0

0

101

0

102

0

11

1

0

5836

141

1096

0

1165

133

107

139

0

0

46

1

0

17

0

46

0

18

196

92

10

17

0

489

0

78

0

438

544

2

0

0

1114

0

0

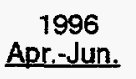

Apr.-Jun.

0
0
1566
0
216
0
0
0
10
324
0
1
0
21
0
7298

1216

1419

83

1593

0

80

14

58

893

0

0

0

0

155

27

29

0

0

77

75

0

0

204

0

0

0

801

1101

0

27

0

0

126
1996

Jul.-Sep.

0

0
768

0

0

9

0

35

0

491

408

1

0

5

0

6326

289

1259

0

1884

0

104

97

4

767

0

0

0

337

0

21

0

0

14

92

80

44

2

155

46

0

0

474

494

0

554

119

0

0

11
1997

Oct.-Dec. Jan.-Mar.

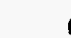

382

0

0

0

4

35

110

667

362

9

21

20

9

4389

138

957

0

1598

0

104

105

0

540

0

0

0

49

0

0

72

0

0

79

23

0

8

0

104

0

0

154

304

0

155

345

0

44

0

25

4458

0

0

0

10

0

0

644

649

10

39

3

48

4626

1054

751

0

1066

107

65

57

0

0

0

0

93

0

0

0

0

334

57

37

280

6

0

68

0

96

248

567

5 
PURCHASERS OF SHORT-TERM GAS Estimated Volumes Imported (MMCF)

CMS NOMECO Oil \& Gas Co.

CNG Energy Services

CNG Gas Services

CNG Producing Company

CNG Transmission Corporation

CPC Intemational

Cabot Oil

CalPine Fuels Corporation

CanStates Gas Marketing

CanStates Petroleum Marketing

Canadian Natural Resources

Canwest Gas Supply

Cardinal Cogen

Camation Dairies

Carthage

Cascade Natural Gas

Catex Vitol

Cavenham

Celcius

Celotex Corporation

Cenergy, Inc.

Cenerprise

Cenex, Inc.

Cenral Premix

Centra Gas Manitoba Inc.

Central Hudson Gas \& Electric

Central Illinois Light Company

Central Southwest

1996

Jan.-Mar

1996

1996

Jul.-Sep

1996

Oct.-Dec.

1997

19

4643

0
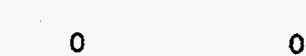

155

Jan.-Mar.

Chandler Energy

Channel Gas Marketing

Chevron Natural Gas Services, Inc.

Chevron Products Company

Chevron Real Estate Company

Chevron Storage

Chevron U.S.A. Inc.

Chooljian Brothers

Cibola Corporation

Cintas

City of Buckley, OR

City of Ellensburg, WA

City of Enumclaw, WA

City of Palo Alto, CA

City of Perham, MN

Clinton Gas Marketing

CoEnergy Trading Company

Coastal Gas Marketing Company

843
19

2564

$$
128
$$

0

366

Colonial Energy, Inc.

Colonial Gas Company 
PURCHASERS OF SHORT-TERM GAS Estimated Volumes Imported (MMCF)

Columbia Beverage Jan.-Mar 1996
Apr-Jun

1996 Jul.-Sep.

1996

Oct.-Dec.

1997 Jan.-Mar.

Columbia Energy Service

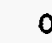

96

Columbia Falls Aluminum

Columbia Natural Gas

Commonwealth Edison

Commonwealth Energy Services

Commonwealth Gas Company

ConAgra Energy

Connecticut Natural Gas

Conoco inc.

Consolidated Edison

Consolidated Energy

Consolidated Natural Gas

Consumers Power Company

Continental Natural Gas

Cook inlet

Coral Energy Resources

Coming, Inc.

Corpus Christi Gas Marketing, L.P.

Cowest Energy

Crockett Cogeneration

Darling Delaware

Davis Wire

Defense Fuel Supply

Del Ray Packing

Destec Gas Services

Direct Energy Marketing

Draper Farms

Dynamis

Dyno Polymers

EPEC Marketing Company

ERI Services

ESCO Corporation

Eagle Gas Marketing

Eagle Picher

Eastem Energy Marketing

Eastem Group

Eastem State Hospital

Eastern Washington University

Eastex Energy

Eastex Hydrocarbons

EI Paso Gas Marketing

Elanbaas

Elizabethtown Gas Company

Emmanual

Empire Natural Gas Corporation

Encina Gas Marketing

Encogen Northwest, L.P.

Energy Source inc.

Energynorth inc.

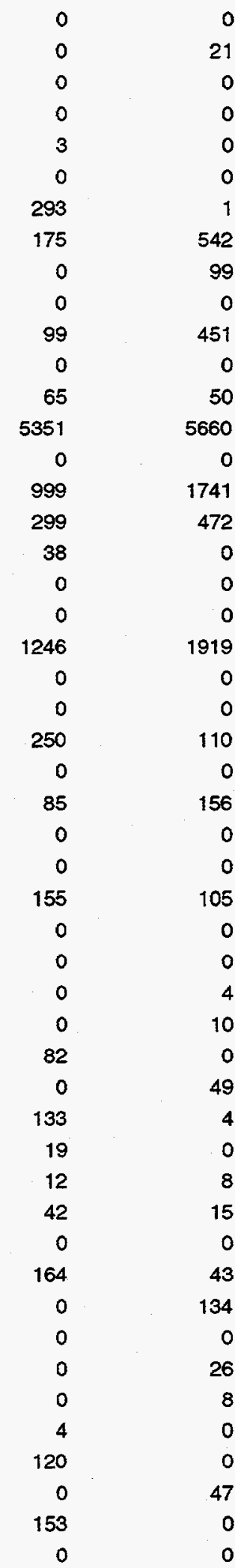

\begin{tabular}{|c|c|}
\hline 0 & 11 \\
\hline 0 & 0 \\
\hline 0 & 0 \\
\hline 0 & 0 \\
\hline 0 & 0 \\
\hline 0 & 12 \\
\hline 99 & 60 \\
\hline 967 & 1078 \\
\hline 652 & 1508 \\
\hline 0 & 5 \\
\hline 524 & 416 \\
\hline 0 & 0 \\
\hline 0 & 0 \\
\hline 4894 & 5442 \\
\hline 0 & 34 \\
\hline 1594 & 2005 \\
\hline 885 & 486 \\
\hline 0 & 0 \\
\hline 16 & 154 \\
\hline 385 & 34 \\
\hline 991 & 834 \\
\hline 0 & 9 \\
\hline 0 & 8 \\
\hline 0 & 0 \\
\hline 3 & 0 \\
\hline 592 & 593 \\
\hline 0 & 0 \\
\hline 3 & 4 \\
\hline 0 & 0 \\
\hline 0 & 6 \\
\hline 0 & 3 \\
\hline 466 & 169 \\
\hline 0 & 6 \\
\hline 400 & 0 \\
\hline 0 & 5 \\
\hline 495 & 1363 \\
\hline 0 & 0 \\
\hline 14 & 13 \\
\hline 33 & 25 \\
\hline 0 & 0 \\
\hline 17 & 0 \\
\hline 1141 & 305 \\
\hline 1 & 3 \\
\hline 0 & 0 \\
\hline 0 & 0 \\
\hline 0 & 28 \\
\hline 0 & 0 \\
\hline 0 & 0 \\
\hline 158 & 117 \\
\hline 122 & 147 \\
\hline
\end{tabular}


Enron Capital \& Trade Resources

Enron Gas Marketing

Enserch Development Corporation

Enserch Energy Services, Inc.

Enserch Gas Company

Enserco Energy, Inc.

Ensource

Equitable Resources Marketing Co.

Essex County Gas Company

Evanite Fiber

Exxon Corporation

Fall River Gas Company

Farmland Industries

Fina Natural Gas

Fircrest Schools

Fitchburg Gas \& Electric Company

Formica Corporation

Fort Lewis

Foster Farms

Fuel Imbalance

Gasmark Inc.

Gaylord Container Corporation

General Chemical

Georgia Pacific Corporation

Georgia-Pacific (West)

Glass Eye

Global Petroleum

Goal Line

Golden Field Green Houses

Golden Spread Energy

Granite State Gas Transmission, Inc.

Great Falis Gas Company

Great Lakes Gas Transmission Co.

Great Plains Natural Gas Company

Greeley Gas Company

Gulf Energy Marketing Company

H\&N Gas

Hadson Gas Marketing

Hadson Gas Systems

Heartiand Energy

Heath

Hermiston Generating Company

Holly Sugar

Hoinam Inc.

Holyoke Gas Company

Home Oil

Howard Energy Company

Hutchinson

IBP

ICC Energy

\begin{tabular}{|c|c|c|c|c|}
\hline $\begin{array}{c}1996 \\
\text { Jan-Mar. }\end{array}$ & $\begin{array}{c}1996 \\
\text { Apr.-Jun. }\end{array}$ & $\begin{array}{c}1996 \\
\text { Jul.-Sep. }\end{array}$ & $\begin{array}{c}1996 \\
\text { Oct.-Dec. }\end{array}$ & $\begin{array}{c}1997 \\
\text { Jan.-Mar. }\end{array}$ \\
\hline 2412 & 12918 & 5094 & 3871 & 2794 \\
\hline 2496 & 5915 & 4998 & 3845 & 2741 \\
\hline 233 & 11 & 0 & 0 & 0 \\
\hline 484 & 924 & 590 & 1768 & 612 \\
\hline 155 & 26 & 0 & 0 & 0 \\
\hline 0 & 0 & 0 & 0 & 532 \\
\hline 0 & 0 & 56 & 0 & 228 \\
\hline 5692 & 7696 & 6944 & 5942 & 5946 \\
\hline 0 & 0 & 35 & 57 & 50 \\
\hline 0 & 0 & 4 & 84 & 42 \\
\hline 1900 & 657 & 950 & 2053 & 2024 \\
\hline 0 & 0 & 0 & 126 & 132 \\
\hline 0 & 0 & 0 & 310 & 0 \\
\hline 34 & 0 & 0 & 0 & 0 \\
\hline 0 & 1 & 0 & 0 & 21 \\
\hline 0 & 0 & 9 & 4 & 10 \\
\hline 85 & 33 & 27 & 0 & 0 \\
\hline 30 & 0 & 0 & 0 & 0 \\
\hline 85 & 67 & 56 & 0 & 0 \\
\hline 0 & 5 & 0 & 122 & 753 \\
\hline 0 & 0 & 0 & 5 & 2 \\
\hline 1117 & 1060 & 974 & 0 & 0 \\
\hline 28 & 0 & 0 & $: 0$ & 8 \\
\hline 130 & 120 & 110 & 118 & 115 \\
\hline 164 & 44 & 52 & 0 & 0 \\
\hline 0 & 0 & 0 & 0 & 1 \\
\hline 496 & 527 & 0 & 61 & 8 \\
\hline 0 & 0 & 0 & 199 & 0 \\
\hline 10 & 2 & 0 & 0 & 0 \\
\hline 0 & 0 & 0 & 0 & 0 \\
\hline 0 & 0 & 0 & 0 & 25 \\
\hline 1005 & 282 & 206 & 398 & 0 \\
\hline 66 & 140 & 154 & 60 & 0 \\
\hline 821 & 431 & 451 & 0 & 0 \\
\hline 17 & 0 & 0 & 63 & 0 \\
\hline 233 & 70 & 0 & 0 & 0 \\
\hline 0 & 38 & 59 & 0 & 0 \\
\hline 168 & 0 & 0 & 0 & 0 \\
\hline 200 & 0 & 0 & 0 & 0 \\
\hline 6 & 0 & 0 & 0 & 0 \\
\hline 0 & 0 & 0 & 0 & 4 \\
\hline 12 & 1523 & 0 & 0 & 0 \\
\hline 35 & 124 & 0 & 0 & 0 \\
\hline 445 & 485 & 531 & 328 & 451 \\
\hline 0 & 0 & 0 & 35 & 24 \\
\hline 878 & 0 & 0 & 0 & 0 \\
\hline 639 & 151 & 1037 & 1252 & 1184 \\
\hline 0 & 0 & 0 & 0 & 68 \\
\hline 57 & 0 & 48 & 62 & 0 \\
\hline 8 & 82 & 0 & 8 & 0 \\
\hline
\end{tabular}


PURCHASERS OF SHORT-TERM GAS Estimated Volumes Imported (MMCF)

IGI Resources

Indeck Energy Services of Oswego

1996

Apr.Jun

Jan.-Mar.

Apr.-Jun

Jul.-Sep

1996

Oct.-Dec.

1997

10862

10897

10114

9253

Jan.-Mar.

Indeck Energy Services of Yerkes

218

279

Industrial Energy Applications

Industrial Energy Services Company

217

219

216

7955

207

0
63

Industrial Heat Treating Company

Inland Natural Gas

Inland Pacific Energy Services Corporation

5

300

0

0

1267

Inland Steel Company

401

Intalco Aluminum Corporation

155

Intercoast

0
1002

Interenergy Corporation

Interox

J. Aaron Company

J.H. Gypsum

J.R. Simplot Company

James River Corporation

Jorgenson Forge

KCS Energy Management

KCS Energy Marketing

KN Energy

KTEA

KTM

Kaiser - Mead

Kaiser Cement

Kaiser-T.W.

Kaltum

Kaztec Energy

Kaztex Energy Management

Kimball Energy Corporation

Kimball Trading Company, L.L.C.

Kings Meat

Koch Energy Trading

Koch Gas Services Company

Kraft General Foods

0

0
2976

1086

0

351

49

14

74

10

0

24

0

453

0

0

59

0

0

0

4485

L'Energia L.P.

L.E. Mason Company

LG\&E Natural Marketing Inc.

Lakeland Village

Lakeside

Lamb Weston

Lane Mountain Silica

Legacy Health System

Lignetics

Lockport Energy

Long Island Lighting Company

Longview Fibre

Louis Dreyfus Energy Corporation

367

0 
M.A. Segale

MTVHS

Madison Gas \& Electric

Marko Foam Products

Martinez Cogeneration

Masonite Corporation

Masspower

Medina Power Company

Medite Corporation

Metropolitan Utilities District

MichCon Trading

Michigan Consolidated Gas Company

Michigan Gas Company

Mid American Natural Resources

MidAmerican Energy Company

Midcon Gas Services Corporation

Midcon Storage

Middleborough Gas Company

Midland Cogeneration Venture

Milford Power, L.P.

Minnegasco

Minnesota Com Processors

Mobil Gas Marketing Inc.

Mobil Natural Gas Inc.

Mobil Oil

Mock Resources

Montana Power

Montana Resources

Morgan Stanley

Mountain Fuel

Mt. Vemon

Murphy Gas Gathering

Murphy Oil

Myshic River

N.Y. State Electric \& Gas

NESI Energy Marketing

NMU Gas Purchasing Inc.

NORPAC

NPGA

NSP

NUI Corporation

Nabisco

National Energy \& Trade, L.L.C.

National Fuel Gas Distribution

National Fuel Gas Supply Corporation

National Fuel Pipeline

National Fuel Resources

National Gas \& Electric

National Gas Resources

National Steel Corporation

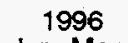

Jan. Mar.

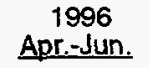

0

0

0

13

0

0

0

158

0

0

1335

2211

205

0

1382

2397

0

0

0

507

2348

0

0

489

9

1584

0

54

0

0

0

296

0

0

45

0

1277

0

0

0

0

95

0

2063

0

54

782

16154

0

383
Apr.-Jun.

0
0
0
10
0
55
0
222
0
0
1355

3610

0

0

463

98

0

0

685

525

1353

0

0

2769

0

1897

0

22

10

0

0

296

0

66

168

0

0

0

0

0

0

0

0

1053

0

0

19103

563

1876
1996

Jul.-Sep.
1996

Oct-Dec.
1997

Jan.-Mar.

2687

0

0

0

7

426

0

86

218

44

186

1380

2687

0
0

468

45

301

11

780

552

914

0

4

10

0

1715

16

0

335

4

0

299

1

0

433

0

0

0

0

0

0

81

0

142

0

0

0

16635

462

2289

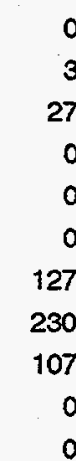

32

6

0

0

0

81

11

223

131

0

0

4794

0

153

966

73

0
22

313

557

2467

0

8

0

1775

2697

80

305

0

0

299

7

0

144

126

0

0

0

0

0

37

29

368

10

0

39

15425

288

1554

8351

0 .

0

1186

168

0

12

230

544

2321

436

0

0

0

621

3536

80

0

134

1

293

0

0

0

0

369

4

27

465

80 
PURCHASERS OF SHORT-TERM GAS Estimated Volumes Imported (MMCF)

Natural Fuel Gas

Natural Gas Clearinghouse

Natural Gas Exchange Inc.

Natural Gas Resources

Natural Gas Transmission

Natural Gas Transportation Services

Naumes

Nestle USA, Inc.

Nevada Power Company

New England Gas Association

New England Power Company

New Jersey Natural Gas

Niagara Mohawk

Ninomiya Nursery

Noram Energy Services

Norcen Energy Resources

Norstar Energy

North American Energy Inc.

North American Resources Company

North Attleboro Gas Company

North Canadian Marketing Corp.

Northeast Energy Associates

Northeast Heat \& Light

Northeast Utilities

Northem California Power Agency

Northem Indiana Public Service

Northem Minnesota Utilities

Northem States Power (MN)

Northem States Power (WI)

Northem States Power Company

Northem Utilities, Inc.

Northshore Gas Company

Northstar Energy

Northwest Alloys

Northwest Cooperage

Northwest Natural Gas Company

Northwest Pipeline

Northwest Public Service

Novagas Clearinghouse

Novergaz 1994 Inc.

Nurserymen's Exchange

Ocean K

Ocean State Power

Ocean State Power II

Omaha Utilities District

Onyx Gas Marketing Company

Orange \& Rockland Utilities

Orchard Gas

Ore-Ida

Oregon Potato
1996

Jan.-Mar.

1996

Apr.-Jun

0

5520

0

141

315

173

0

72

0

0

1053

0

54

32

3764

131

0

7

20

0

147

0

20

0

32

0

1104

0

186

1678

322

3697

0

165

0

2107

0

0

0

0

41

0

18

18

0

11

0

0

30

0

44

0

321

0

72

10

4099

0

125

0

57

0

0

0

3

4

174

0

1107

114

0

1357

0

3687

0

149

0

2142

9

0

0

0

29

0

0

0

186

0

0

0

82

0
1996

Jul. Sep.

Oct-Dec

$\begin{array}{rr}0 & \\ 6734 & 83 \\ 0 & \\ 0 & 3 \\ 0 & 139 \\ 0 & \end{array}$

7
147
1996

1997

Jan-Mar.

0

8346

9970

0

0

100

0

6

0

6

0

2134

75

0

0

2481

0

0

388

0

9

0

0

0

169

29

65

0

0

0

903

40

3217

6

0

16

1302

0

576

0

0

0

1

82

0

446

441

0

247

10

15 
PURCHASERS OF SHORT-TERM GAS

Estimated Volumes Imported (MMCF)

1996

Jan.-Mar.

Oroweat

Overall Laundry

$P \& N$, L.P.

PABST

PG\&E Gas Supply Business Unit

PITCO

Pacific Gas \& Electric (UEG)

Pacific Gas \& Electric Company

Pacific Gas Transmission

Pacific Gas Transmission (Line Pack)

PanEnergy Gas Services, Inc.

PanEnergy Trading \& Marketing Svcs., Inc., L.L.C.

Panhandle Gas Services

Paragon Gas Marketing

Pawtucket Power Associates

Pendelton

Pendulum

Penn Union Energy

Penwest

Peoples Gas, Light, \& Coke

Peoples Natural Gas Company

Petro Canada Hydrocarbons

Petro Source Gas Ventures

Phibro Division of Salmon

Phibro Energy USA Inc.

Phibro Oil \& Gas Inc.

Phoenix Chemical

Phoenix Soil

Piedmont Natural Gas Company

Pierce Transit

Pittsfield Generating

Plum Creek Manufacturing

Poco Petroleum

Ponderosa

Portland Adventist

Portland General Electric Company

Pottatch Corporation

Potsdam College

Potters Industries

Proctor and Gamble Company

Progas

Promark Energy

Puget Sound Energy

Puget Sound Power \& Light

Questar Energy Trading Co.

Quick Trade

R.C. Collet, Inc.

Rainbow Gas Company

Rainy River

Ranck Oil Company
1996

Apr.-Jun

Apr.-Jun

.

0
49584

0

188

2227

100

0

20617

0

2108

0

19

0

5

0

0

2789

0

1745

313

149

110

0

0

38

0

0

66

0

1860

1

48

64

299

38

83

0

0

968

0

5

1

0

0

47219

15252

0

1305

21

4

0

16

0

0

2876

433

12

0

0

781

41

302

0

0

0

0

52

0

0

0

107

3

47

63

13

585

813

0

144

361

0

6

409

0

4
1996

Jul.-Sep.

Jul.-Sep

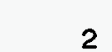

$\begin{array}{rr}0 & 2 \\ 0 & 0 \\ 40 & 0 \\ 10 & 0\end{array}$

46570

21885
1996
Oct.-Dec.

0

647

7374

49

0

1997 Jan-Mar.

$\begin{array}{rr}0 & 1 \\ 0 & 8 \\ 0 & 0 \\ 0 & 34 \\ 52366 & 52784 \\ 0 & 422 \\ 525 & 147 \\ 3696 & 3215 \\ 0 & 0 \\ 190 & 0 \\ 1280 & 352 \\ 27247 & 30183\end{array}$

609

1116

0

8

5

0

358

3

3056

155

460

0

0

0

0

149

457

0

0

0

26

0

0
7

5294

24

48

65

65

0

41

0
0

476

0

5

6

0
1

8

34

784

422

147

15

0

352
0183

0

0

11

19

0

152

0

2835

0

522

221

0

111

448

6

208

9

15

0

1

1

0
4394

0

47

0

0

0

0

790

0

783

143

0

0

19

9 
PURCHASERS OF SHORT-TERM GAS Estimated Volumes Imported (MMCF)

Redhook

Redwood Resources Inc.

Renaissance Energy (U.S.) Inc.

Jan.-Mar. $-$

Rhone-Poulenc

Ripon Cogeneration

Rochester Gas \& Electric Company

Roseburg Lumber

SCPL

SEMCO Energy Services

SUNY - N.Y.

Sacramento Municipal Utility Dist.

San Diego Gas \& Electric Company

Santa Clara Co. Gen. Svcs.

Santa Clara Co. Med. Ctr.

Schuller Intemational

Seattle Snohomish

Seitel Gas \& Energy Corporation

Selkirk Cogeneration Partners

Shell Gas Trading Company

Shell Oil Company

Sierra Pacific Industries

Sierra Pacific Power Company

Simpson Paper

Simpson Tacoma Kraft Company

Smurfit Newsprint Corporation

Sonat Marketing Company

South Jersey Gas Company

Southeastem Michigan Gas Company

Southem Cajifomia Edison Company

Southem Califomia Gas Company

Southem Connecticut Gas Company

Southward Energy Ltd.

Southwest Gas Corporation

Specialty Aluminum Company

Sprague Energy

St. Agnes Medical Center

St. Lawrence Gas

St. Lawrence University

StampGas

Stand Energy

State University of New York

Stone Container Corporation

Storage in IA

Storage in IL

Storage in PA

Storage in WA

Sumas Cogeneration

Superior

Supreme Energy Company

Syar
1996

1996
Apr.-Jun.

1996
Jull.-Sep.

0
820

13352

94

0

32

17

644

137

1361

198

710

66

41

91

0

0

599

158

16

1695

9173

197

270

0

740

0

176

3022

0

0

0

1262

0

16

49

33

33

38

471

27

447

0

2

248

38

16

0

0

0

$\begin{array}{rr}0 & \\ 1795 & \\ 9185 & 88 \\ 78 & \\ 0 & \end{array}$

36
881
9

363

11

99
0

0
17

17
0

37

929

741

1026

21

17

38

0

7
135

0

0

0

391
7265

166

299

0

0

0

118

3586

41

0

128

769

0

0

33

0
33

33
0

73

0

287

1

0

6

62

0

0

0

7440

20
288

288
0

884

0

0

4907

34

65

0

623

1

0
34

106

22

0

0

0

500

0

0

0

0

0

0

0
1996

Oct.-Dec.

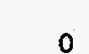

175

8829

89

288

900

17

0

2

987

246

2809

0

0

53

0

1005

0

0

0

37

9541

88

368

0

180

19

0

3275

34

41

0

718

1

0

0

95

33

422

31

0

498

0

0

0

0

48

0

1

0
1997

Jan.-Mar.

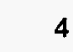

277

9221

28

507

2715

34

0

802

968

181

2923

0

0

o

9

0

432

0

0

0
0022

4
1
7
5
4
0
2
1
3
0
0
0
9
0
2
0
0
0

10022

0

44

28

0

14

0

3486

0

30

0

971

0

0

0

0

33

622

67

0

396

0

0

0

0

44

142

1 
PURCHASERS OF SHORT-TERM GAS

Estimated Volumes Imported (MMCF)

Syracuse China Company

TGP Storage

Talisman Energy

Tarpon Gas Marketing

Tecnal

Teco Gas Marketing

Tejas

Teledyne

Tenaska Gas Company

Tenaska Marketing Ventures

Tenneco Gas Marketing

Tennessee Gas Pipeline Company

Terra International

Texaco Gas Marketing Inc.

Texaco Natural Gas

Texas Eastem Transmission

Texas-Ohio Gas, Inc.

Texia Energy

The Eastem Group

The Montana Power Company

Todds Shipyards

Tosco Energy

Tosco Northwest

Tosco Refining Company

TransCanada Gas Services

TransOcean Products, inc.

Travis Pattem

Trebor Energy Resources

Twister Gas Services

Twister Transmission Company

U.S. Energy Services

U.S. Gas Transportation Inc.

UCLA Cogeneration

US Gas Transmission

US Generating

US Oil \& Refining

USGT Investments

Union Pacific Fuels

Union Pacific Resources

Unisea Foods

United Cogen

Universal Resources

Unocal

Utilicorp Retail Services

Utilicorp United

Valero Gas Marketing

Valley Gas Company

Valley Medical

Various CA State Markets

Various CO State Markets
1996

Jan.-Mar

1996

Apr.-Jun

35

0

0

4

0

60

0

0

3847

313

0

923

119

731

2

11

3

0

1861

0

238

0

2664

0

27

30

0

112

0

1421

720

361

0

0

0

857

0
0

439

0

0

0

2680

1707

0

11801

0

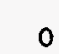

573

16

0

0

325

0

0

0

5596

21

0

920

31

357

231

2

0

54

1063

0

9

126

0

2295

0

25

0

0

0

30

605

705

0

0

12

0

1034

0

0

366

0

414

0

0

2081

62

0

8941

0
1996
Jul.-Sep.

1996
Oct.-Dec.

1997

Jan.Mar.

\section{0}$$
0
$$$$
0
$$$$
0
$$$$
0
$$$$
0
$$$$
0
$$$$
0
$$

3655

96

331

861

19

74

0

1

$$
0
$$$$
0
$$$$
0
$$$$
0
$$

.


PURCHASERS OF SHORT-TERM GAS Estimated Volumes Imported (MMCF)

Various IA State Markets Various IL State Markets Various IN State Markets Various KY State Markets Various MI State Markets Various MN State Markets Various MT State Markets Various NE State Markets Various NJ State Markets Various NY State Markets Various OH State Markets Various PA State Markets

Various RI State Marketes Various TX State Markets Various WA State Markets Various WI State Markets Various WV State Markets Vastar Gas

Vermont Gas Systems, Inc. Vesta Energy Company Vitol Gas \& Electric WP Natural Gas WPS Energy Services, Inc.

Wasatch Oil \& Gas

Wascana Energy Marketing

Washington Energy Gas Marketing Co.

Washington Natural Gas Company

Washington Potato Company

Washington Water Power Company

Watson Cogeneration

Westar Marketing Company

Westcoast Energy Inc.

Westcoast Gas Services (U.S.A.) Inc.

Westcoast Gas Services Inc.

Westcoast Gas Svcs. (America), Inc.

Western Gas Res. (Dry Creek Storage)

Western Gas Resources

Western Gas Services

Weyerhauser

Whitman College

Wickford Energy Marketing Inc.

Willamette

Williams Energy Company

Williams Gas Marketing

Winchester Hospital

Windward Energy \& Marketing Inc.

Wisconsin Electric

Wisconsin Gas

Wisconsin Power \& Light

Wisconsin Public Service Corp.
1996

Jan.-Mar.

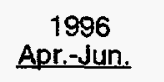

1996
Jul.-Sep.
1996
Oct.-Dec.

1997

2512

251

$\begin{array}{rr}2512 & 73 \\ 0 & 185 \\ 0 & \\ 30 & \\ 0 & 271\end{array}$

194

0

10228

0

297

0

0

785

672

823

20

209

50

0

1715

0

147

0
231

7960

59

3825

520

0

0
1110

1110

703

8345

0

0

0

726

0

531

353

29

0

0

0
92

2150

0
73

185

0

271

1004

567
3031
112
185
190
9819

668
1924
3619
157

684

0

262

0

16

3903

51

4680

812

3382
1143

0

581

534

1202

4870

145

910

0

34

2

113

837

455

5

0

7

0

182

1014

0

6863

0

466

0

238

0

147

677

177

0

2
0

0

93

1025

0
821

0

476

6830

1755

301

0

15292

0

0

0

323

2937

304

0

161

0

0

157

357

0

100

832

3

6394

62

6181

498

1

303

642

926

6141

225
Jan.-Mar.

$$
\begin{array}{r}
4700 \\
0 \\
0 \\
0 \\
7539 \\
1163 \\
1055 \\
0 \\
0 \\
16236 \\
0 \\
0
\end{array}
$$

0

881

8639

0

465

267

0

0

312

153

253

1840

21

5152

60

5208

0

0

227

1270

261

5583

0

221

0

358

0

152

869

1030

0

2

0

217

91

1313

13 


\section{PURCHASERS OF SHORT-TERM GAS}

Estimated Volumes imported (MMCF)

Wollaston Alloys, Inc.

Woodworth

Wyoming Industrial Gas

$X$ Energy, Inc.

Yankee Gas Services

Totals

\begin{tabular}{rr}
$\begin{array}{r}1996 \\
\text { Jan.Mar. }\end{array}$ & $\begin{array}{r}1996 \\
\text { Apr.-Jun }\end{array}$ \\
0 & 0 \\
0 & 0 \\
13 & 0 \\
0 & 0 \\
93 & 0 \\
372816 & 363073 \\
\hline
\end{tabular}

1996
Apr.-Jun

0

0 $\underline{363073}$
1996

Jul.-Sep.

2
0
0
17
0

$\underline{354056}$
1996

Oct.-Dec.

2
0
0
36
0

$\underline{382168}$
1997 Jan.-Mar.

1
16
0
21
47

$\underline{399549}$ 
SHORT-TERM SALES

YEAR: 1997 Quarter: One

Estimated Volumes (MMCF) \& Prices (\$ / MMBTU)
Importer

Seller

Purchaser / End User

AEC WEST LTD.

Conwest Exploration Company Limited Enron Gas Marketing

AIG TRADING CORPORATION

PEMEX

AIG Trading Corporation

Hidaigo, Texas

AIG TRADING CORPORATION

Various Suppliers

Michigan Consolidated Gas Company

AIG TRADING CORPORATION

Various Suppliers

Michigan Consolidated Gas Company

AIG TRADING CORPORATION

Various Suppliers

AIG TRADING CORPORATION

Various Suppliers

Pacific Gas \& Electric Company

ALENCO GAS SERVICES INC.

AEC Oil \& Gas Company

Amoco Trading

Eastport, ldaho

Eastport, Idaho

ALENCO GAS SERVICES INC.

AEC Oil \& Gas Company

Enron Capital \& Trade Resources

ALENCO GAS SERVICES INC.

$A E C$ Oil \& Gas Company

Equitable Resources Marketing Co.

ALENCO GAS SERVICES INC.

AEC Oil \& Gas Company

Pacific Gas \& Electric Company

Point
of
Entry

Eastport, Idaho

ASERVICES INC.

Coastal Gas Marketing Company
Michigan Consolidated Gas Company

Noyes, Minnesota

St. Clair, Michigan

Waddington, NY

January

Vol. Price

February

Vol. Price

March

Vol. Price

Quarterly

Total

Avg. Price

Port of Morgan, MT

447

3.74

414

2.58

458

1.13

1319

2.47

- N/A

- N/A

$39 \quad 1.87$

39

1.87

623

3.22

255

3.03

$0 \quad N / A$

878

3.16

586

3.49

24

3.26

- N/A

609

3.48

- N/A

$7 \quad 3.03$

- N/A

$7 \quad 3.03$

AEC Oil \& Gas Company

580

2.09

425

1.42

467

$90 \quad 2.41$

357

2.33

- N/A

$\begin{array}{ll}71 & 2.07\end{array}$

$0 \quad$ N/A

$\begin{array}{ll}71 & 2.07\end{array}$
Eastport, Idaho
Eastport, Idaho
284

1.37

31

1.41

894

1.41

Eastport, Idaho

30

4.62

142

2.08

107

1.12

279

1.99
$14 \quad 1.66$

15

1.13

186

1.30 
SHORT-TERM SALES

YEAR: 1997 Quarter: One

Estimated Volumes (MMCF) \& Prices (\$ / MMBTU)
Importer

Seller

Purchaser / End User

Point

Entry

Eastport, Idaho

Massena, New York

Renaissance Energy Ltd

Aluminum Company of America

ALUMINUM COMPANY OF AMERICA

TransCanada Gas Services Limited

Aluminum Company of America

Massena, New York

133

January

Vol. Price

February
Vol. Price

Vol. Price

March

Price

Quarterly

Total

Avg.

Vol. Price

ALENCO GAS SERVICES INC.

ALENCO GAS SERVICESII

TransCanada Gas Services

$19 \quad 4.71$

$17 \quad 3.33$

19

2.34

54

3.46

AMOCO CANADA MARKETING CORPORATION

Amoco Canada Petroleum Company Ltd.

AIG Trading Corporation

Eastport, Idaho

$0 \quad$ N/A

$5 \quad 1.98$

$0 \quad$ N/A

5

1.98

AMOCO CANADA MARKETING CORPORATION

Amoco Canada Petroleum Company Ltd.

Amoco Trading

Eastport, Idaho

112

1.98

1511

1.39

3730

2.06

AMOCO CANADA MARKETING CORPORATION

Amoco Canada Petroleum Company Ltd.

Canwest Gas Supply

Eastport, Idaho

$0 \quad$ N/A

$5 \quad 1.98$

$4 \quad 1.39$

9

1.73

AMOCO CANADA MARKETING CORPORATION

Amoco Canada Petroleum Company Ltd.

Cook Inlet

Eastport, Idaho

43.03

0

N/A

62

1.39

66

1.49

AMOCO CANADA MARKETING CORPORATION

Amoco Canada Petroleum Company Ltd.

Crockett Cogeneration

Eastport, Idaho

306

3.03

289

1.98

239

1.39

834

2.20

AMOCO CANADA MARKETING CORPORATION

Amoco Canada Petroleum Company Ltd.

Enron Gas Marketing

Eastport, Idaho

$8 \quad 3.03$

$0 \quad$ N/A

$0 \quad N / A$

3.03

AMOCO CANADA MARKETING CORPORATION

Amoco Canada Petroleum Company Ltd.

Enserco Energy, Inc.

Eastport, Idaho

$0 \quad N / A$

$0 \quad$ N/A

85

1.39

85

1.39

AMOCO CANADA MARKETING CORPORATION

Amoco Canada Petroleum Company Ltd.

IGI Resources

Eastport, Idaho

$\begin{array}{llllllll}0 & \text { N/A } & 282 & 1.98 & 235 & 1.39 & 517 & 1.71\end{array}$


SHORT-TERM SALES

YEAR: 1997 Quarter: One

Estimated Volumes (MMCF) \& Prices (\$ / MMBTU)
Importer

Seller

Purchaser / End User
Point

of

Entry
Vol Price

$4 \quad 3.03$

Eastport, Idaho

Amoco Canada Petroleum Company Ltd.

Inland Pacific Energy Services Corp

AMOCO CANADA MARKETING CORPORATION

Amoco Canada Petroleum Company Ltd.

Natural Gas Clearinghouse

Eastport, Idaho

AMOCO CANADA MARKETING CORPORATION

Amoco Canada Petroleum Company Ltd.

Noram Energy Services

Eastport, Idaho

AMOCO CANADA MARKETING CORPORATION

Amoco Canada Petroleum Company Ltd.

Northwest Natural Gas Company

Eastport, Idaho

AMOCO CANADA MARKETING CORPORATION

Amoco Canada Petroleum Company Ltd.

Pacific Gas \& Electric Company

Eastport, Idaho

AMOCO CANADA MARKETING CORPORATION

Amoco Canada Petroleum Company Ltd.

PanEnergy Trading \& Marketing Svcs.

Eastport, Idaho

AMOCO CANADA MARKETING CORPORATION

Amoco Canada Petroleum Company Ltd.

Sacramento Municipal Utility Dist.

Eastport, Idaho

AMOCO CANADA MARKETING CORPORATION

Amoco Canada Petroleum Company Ltd.

Sierra Pacific Power Company

Eastport, Idaho

AMOCO CANADA MARKETING CORPORATION

Amoco Canada Petroleum Company Lid.

TransCanada Gas Services

Eastport, Idaho

AMOCO CANADA MARKETING CORPORATION

Amoco Canada Petroleum Company Ltd.

U.S. Gas Transportation Inc.

Eastport, Idaho

AMOCO CANADA MARKETING CORPORATION

Amoco Canada Petroleum Company Ltd.

Wascana Energy Marketing

Eastport, Idaho

February
Vol. Price

March

Vol. Price

Quarterly

Total

Aol. Price 


\section{SHORT-TERM SALES \\ YEAR: 1997 Quarter: One}

Estimated Volumes (MMCF) \& Prices ( $\$$ / MMBTU)

Importer

Purchaser/ End User
Point

Entry

Hidalgo, Texas

Hidalgo, Texas

ANADARKO TRADING COMPANY

PEMEX

Anadarko Trading Company.

AMOCO ENERGY TRADING CORPORATION

PEMEX

Amoco Trading
January

Vol. Price
February

Vol. Price
March

Vol. Price
Quarterly
Total
Avg.
Vol. Price

255

3.16

313

2.18

10

1.81

578

2.61

AQUILA ENERGY MARKETING CORPORATION

Aquila Canada Corporation

Cascade Natural Gas

Eastport, Idaho

$0 \quad$ N/A

o N/A

10

1.17

109

1.17

AQUILA ENERGY MARKETING CORPORATION

Aquila Canada Corporation

Coastal Gas Marketing Company

Eastport, Idaho

O N/A

- N/A

$\begin{array}{ll}48 & 1.17\end{array}$

48

1.17

AQUILA ENERGY MARKETING CORPORATION

Aquila Canada Corporation

Enserco Energy, Inc.

Eastport, Idaho

$83 \quad 2.40$

$124 \quad 2.40$

$3 \quad 1.17$

210

2.38

AQUILA ENERGY MARKETING CORPORATION

Aquila Canada Corporation

Eastport, Idaho

$104 \quad 2.40$

- N/A

- N/A

104

2.40

AQUILA ENERGY MARKETING CORPORATION

Aquila Canada Corporation

U.S. Gas Transportation Inc.

Eastport, Idaho

O N/A

N N/A

$17 \quad 1.20$

17

1.20

AQUILA ENERGY MARKETING CORPORATION

Aquila Canada Corporation

Utilicorp Retail Services

Eastport, Idaho

2.40

$93 \quad 2.40$

$0 \quad$ N/A

203

2.40

AQUILA ENERGY MARKETING CORPORATION

Cascade Natural Gas Corporation

Enserco Energy, Inc.

Eastport, Idaho

O N/A

$17 \quad 1.84$

O N/A

$17 \quad 1.84$

AQUILA ENERGY MARKETING CORPORATION

Cascade Natural Gas Corporation

U.S. Gas Transportation Inc.

Sumas, Washington

$0 \quad$ N/A

$2 \quad 2.05$

o N/A

$2 \quad 2.05$

AQUILA ENERGY MARKETING CORPORATION

Cascade Natural Gas Corporation

Washington Water Power Company

Sumas, Washington

O N/A

$2 \quad 2.05$

- N/A

$2 \quad 2.05$ 


\section{SHORT-TERM SALES \\ YEAR: 1997 Quarter: One \\ Estimated Volumes (MMCF) \& Prices ( $\$$ /MMBTU)}

Importer

Seller

Purchaser/End User

ARCO PRODUCTS COMPANY

CanWest Marketing

Arco Products Company

ARCO PRODUCTS COMPANY Coastal Gas Marketing, Canada Arco Products Company

ARCO PRODUCTS COMPANY Inland Pacific Energy Services Ltd. Arco Products Company

ARCO PRODUCTS COMPANY Natural Gas Clearinghouse Arco Products Company

ARCO PRODUCTS COMPANY PanEnergy Marketing Canada Arco Products Company

ARCO PRODUCTS COMPANY Petro-Canada Hydrocarbons Inc. Arco Products Company

ARCO PRODUCTS COMPANY Westcoast Gas Services Inc. Arco Products Company

BOUNDARY GAS, INC. CoEnergy Trading Company Berkshire Gas Company

BOUNDARY GAS, INC.

CoEnergy Trading Company Boston Gas Company

BOUNDARY GAS, INC.

CoEnergy Trading Company

Connecticut Natural Gas

BOUNDARY GAS, INC.

CoEnergy Trading Company Consolidated Edison

Point

Point

Entry

Sumas, Washington

Sumas, Washington

Sumas; Washington

Sumas, Washington

$19 \quad 2.68$

$0 \quad$ N/A

$0 \quad$ N/A

19

2.68

Sumas, Washington

103

2.39

106

1.06

357

2.70

Sumas, Washington

$4 \quad 4.22$

- N/A

$0 \quad N / A$

$4 \quad 4.22$

Niagara Falls, NY

O N/A

$2 \quad 2.20$

$0 \quad$ N/A

2

2.20

Niagara Falls, NY

- N/A

$17 \quad 2.20$

- N/A

17

2.20

Niagara Falls, NY

- N/A

$3 \quad 2.20$

N/A

3

2.20
Niagara Falls, NY
- N/A

$8 \quad 2.20$

o N/A

8

2.20 
SHORT-TERM SALES

YEAR: 1997 Quarter: One

Estimated Volumes (MMCF) \& Prices (\$ / MMBTU)
Importer

Seller

Purchaser/End User

BOUNDARY GAS, INC

CoEnergy Trading Company

Energynorth Inc.

BOUNDARY GAS, INC.

CoEnergy Trading Company

Essex County Gas Company

BOUNDARY GAS, INC.

CoEnergy Trading Company

Fitchburg Gas \& Electric Company

BOUNDARY GAS, INC.

CoEnergy Trading Company

Granite State Gas Transmission, Inc

Niagara Falls, NY

$0 \quad$ N/A

21

2.20

- N/A

21

2.20

BOUNDARY GAS, INC.

CoEnergy Trading Company

Long Island Lighting Company

BOUNDARY GAS, INC

CoEnergy Trading Company

Valley Gas Company

Niagara Falls, NY

- N/A

$2 \quad 2.20$

O N/A

2

2.20

BOUNDARY GAS, INC

CoEnergy Trading Company

Yankee Gas Services

Niagara Falls, NY

- N/A

16

2.20

o N/A

16

2.20

BOUNDARY GAS, INC

Wascana Energy Marketing Inc.

Boston Gas Company

Niagara Falls, NY

- N/A

3

2.10

- N/A

3

2.10

BOUNDARY GAS, INC

Wascana Energy Marketing Inc.

Connecticut Natural Gas

Niagara Falls, NY

$0 \quad N / A$

12.10

$0 \quad$ N/A

1

2.10

BOUNDARY GAS, INC

Wascana Energy Marketing Inc.

Consolidated Edison

Niagara Falls, NY

$0 \quad$ N/A

$2 \quad 2.10$

- N/A

$2 \quad 2.10$

BOUNDARY GAS, INC

Wascana Energy Marketing Inc.

Energynorth Ine.
Niagara Falls, NY
$1 \quad 2.10$
N/A

2.10 
SHORT-TERM SALES

YEAR: 1997 Quarter: One

Estimated Volumes (MMCF) \& Prices (\$ / MMBTU)
Importer

Seller

Purchaser / End User

Point

of

Entry

Niagara Falls, NY

Wascana Energy Marketing Inc.

Granite State Gas Transmission, Inc

BOUNDARY GAS, INC.

Wascana Energy Marketing Inc.

Long Island Lighting Company
January
Vol. Price

February

Vol. Price

March

Vol. Price

Quarterty

Total

Avg.

Vol. Price

BOUNDARY GAS, INC

Wascana Energy Marketing Inc.

Yankee Gas Services

Niagara Falls, NY

$0 \quad$ N/A

$3 \quad 2.10$

O N/A

3

2.10

CABOT OIL \& GAS MARKETING CORPORATION

AIG Trading Corporation

Various WA State Markets

Sumas, Washington

$6 \quad 3.20$

- N/A

- N/A

6

3.20

CABOT OIL \& GAS MARKETING CORPORATION

Cook Inlet Energy Supply

Various WA State Markets

Sumas, Washington

O N/A

137

2.82

$0 \quad$ N/A

137

2.82

CABOT OIL \& GAS MARKETING CORPORATION

Inland Pacific Energy Services Ltd.

Various WA State Markets

Sumas, Washington

$12 \quad 2.32$

O N/A

$93 \quad 1.09$

104

1.23

CABOT OIL \& GAS MARKETING CORPORATION

Natural Gas Clearinghouse

Various WA State Markets

Sumas, Washington

$0 \quad$ N/A

o N/A

$62 \quad 1.10$

62

1.10

CABOT OIL \& GAS MARKETING CORPORATION

PanEnergy Gas Services, Inc.

Various WA State Markets

Sumas, Washington

$3 \quad 3.40$

- N/A

O N/A

3

3.40

CABOT OIL \& GAS MARKETING CORPORATION

Portland General Electric

Boeing Company

Sumas, Washington

$155 \quad 4.10$

O N/A

$0 \quad$ N/A

155

4.10

CANWEST GAS SUPPLY U.S.A., INC.

CanWest Gas Supply Inc.

Amoco Trading

Sumas, Washington

$3 \quad 0.95$

$0 \quad$ N/A

$0 \quad N / A$

3

0.95

CANWEST GAS SUPPLY U.S.A., INC

CanWest Gas Supply Inc.

Arco Products Company

Sumas, Washington

- N/A

20.89

$0 \quad$ N/A

2

0.89 


\section{SHORT-TERM SALES \\ YEAR: 1997 Quarter: One \\ Estimated Volumes (MMCF) \& Prices (\$ / MMBTU)}

Importer

Seller

Purchaser/End User

CANWEST GAS SUPPLY U.S.A., INC. CanWest Gas Supply Inc.

Cascade Natural Gas

CANWEST GAS SUPPLY U.S.A., INC. CanWest Gas Supply Inc.

Coastal Gas Marketing Company

CANWEST GAS SUPPLY U.S.A., INC.

CanWest Gas Supply Inc.

ConAgra Energy

CANWEST GAS SUPPLY U.S.A., INC. CanWest Gas Supply Inc.

Cook Inlet

Sumas, Washington

CANWEST GAS SUPPLYU.S.A., INC. CanWest Gas Supply Inc.

Destec Gas Services

CANWEST GAS SUPPLY U.S.A., INC. CanWest Gas Supply Inc.

Enron Capital \& Trade Resources

CANWEST GAS SUPPLY U.S.A., INC CanWest Gas Supply Inc.

IGI Resources

Sumas, Washington

$604 \quad 0.95$

970

0.89

121

0.83

2787

0.88

CANWEST GAS SUPPLYU.S.A., INC. CanWest Gas Supply Inc.

James River Corporation

CANWEST GAS SUPPLY U.S.A., INC. CanWest Gas Supply Inc.

Kimball Energy Corporation

CANWEST GAS SUPPLYU.S.A., INC CanWest Gas Supply Inc.

Masonite Corporation

Sumas, Washington

O N/A

o N/A

81

0.83

81

0.83

CANWEST GAS SUPPLY U.S.A., INC. CanWest Gas Supply Inc. Medite Corporation
Sumas, Washington
Quarterly

Avg.

Vol. ${ }^{\text {Avg. }}$ Price

$43 \quad 0.95$

421

863

0.89

Sumas, Washington

$202 \quad 0.95$

25

33

0.83

792

0.88

Sumas, Washington

$10 \quad 0.95$

$10 \quad 0.89$

o. N/A

20

0.92

$50 \quad 0.95$

39

0.89

42

0.83

131

0.89 
SHORT-TERM SALES

YEAR: 1997 Quarter: One

Estimated Volumes (MMCF) \& Prices (\$ / MMBTU)
Importer

Seller

Purchaser / End User

CANWEST GAS SUPPLY U.S.A., INC.

CanWest Gas Supply Inc.

National Gas \& Electric

CANWEST GAS SUPPLYU.S.A., INC.

CanWest Gas Supply Inc.

Natural Gas Clearinghouse

CANWEST GAS SUPPLY U.S.A., INC.

CanWest Gas Supply Inc.

Nevada Power Company

CANWEST GAS SUPPLY U.S.A., INC. CanWest Gas Supply Inc.

Noram Energy Services

CANWEST GAS SUPPLY U.S.A., INC.

CanWest Gas Supply Inc.

Northwest Natural Gas Company

CANWEST GAS SUPPLY U.S.A., INC. CanWest Gas Supply Inc.

Pacific Gas \& Electric Company

CANWEST GAS SUPPLY U.S.A., INC. CanWest Gas Supply Inc.

PanEnergy Gas Services, Inc.

CANWEST GAS SUPPLY U.S.A., INC.

CanWest Gas Supply Inc.

Portland General Electric Company

CANWEST GAS SUPPLY U.S.A., INC.

CanWest Gas Supply Inc

Questar Energy Trading Co.

CANWEST GAS SUPPLY U.S.A., INC. CanWest Gas Supply inc.

Ripon Cogeneration

Sumas, Washington

251

0.95

January

February

Vol. Price

March

Vol. Price

Quarterty
Total

Avg.

Vol. Price

Sumas, Washington

263

0.89

149

0.83

548

0.89

Sumas, Washington

$5 \quad 0.89$

- N/A

42

0.94

Sumas, Washington

o N/A

o N/A

6

0.95

Sumas, Washington

$2 \quad 0.89$

- N/A

19

0.94

Sumas, Washington

258

0.89

- N/A

1007

0.93

Sumas, Washington

$7 \quad 0.89$

23

0.83

339

0.94

Sumas, Washington

Sumas, Washington

O N/A

O N/A

1

0.95

CANWEST GAS SUPPLY U.S.A., INC. CanWest Gas Supply inc.

Sierra Pacific Power Company

Sumas, Washington
$39 \quad 0.95$

$6 \quad 0.89$

- N/A

45

0.94 


\section{SHORT-TERM SALES \\ YEAR: 1997 Quarter: One}

Estimated Volumes (MMCF) \& Prices (\$ / MMBTU)

Importer

Seller

Purchaser / End User

CANWEST GAS SUPPLY U.S.A., INC.

CanWest Gas Supply inc.

Simpson Tacoma Kraft Company

CANWEST GAS SUPPLY U.S.A., INC.

CanWest Gas Supply inc.

Southwest Gas Comporation

CANWEST GAS SUPPLY U.S.A., INC.

CanWest Gas Supply Inc.

Texaco Gas Marketing Inc.

CANWEST GAS SUPPLY U.S.A., INC.

CanWest Gas Supply Inc.

Tosco Northwest

Sumas, Washington

January

of

Entry

Sumas, Washington

Sumas, Washington

Sumas, Washington

$235 \quad 0.95$

o N/A

N/A

235

0.95

CANWEST GAS SUPPLY U.S.A., INC.

CanWest Gas Supply inc.

UCLA Cogeneration

CANWEST GAS SUPPLY U.S.A., INC. CanWest Gas Supply Inc.

US Gas Transmission

CANWEST GAS SUPPLY U.S.A., INC.

CanWest Gas Supply Inc.

United Cogen

CANWEST GAS SUPPLY U.S.A., INC. CanWest Gas Supply inc.

Wasatch Oil \& Gas

CANWEST GAS SUPPLY U.S.A., INC.

CanWest Gas Supply Inc.

Washington Natural Gas Company

CANWEST GAS SUPPLY U.S.A., INC.

CanWest Gas Supply inc.

Western Gas Resources

Sumas, Washington

$9 \quad 0.95$

o. N/A

N/A

$9 \quad 0.95$

CASCADE NATURAL GAS CORPORATION Aquila Energy Marketing

Cascade Natural Gas

Eastport, Idaho

Quarterly
Total
Avg.
Vol Price

44 0.94

Sumas, Washington

$193 \quad 0.95$

o N/A

- N/A

193

0.95

Sumas, Washington

$275 \quad 0.95$

$33 \quad 0.89$

$19 \quad 0.83$

327

0.94

Sumas, Washington

$173 \quad 0.95$

$0 \quad N / A$

- N/A

173

0.95

Sumas, Washington

$105 \quad 0.95$

$67 \quad 0.89$

$81 \quad 0.83$

253

0.90

Sumas, Washington

$1185 \quad 0.95$

$1030 \quad 0.89$

1193

0.83

3408

0.89

o N/A

o N/A

106

1.18

106

1.18 


\section{SHORT-TERM SALES}

YEAR: 1997 Quarter: One

Estimated Volumes (MMCF) \& Prices (\$ / MMBTU)

Importer

Seller

Purchaser/End User

CASCADE NATURAL GAS CORPORATION Aquila Energy Marketing

Cascade Natural Gas

CASCADE NATURAL GAS CORPORATION

ERI Energy Supply \& Trading

Cascade Natural Gas

Point

of

Entry

Sumas, Washington

$0 \quad$ N/A

10

1.47

$0 \quad$ N/A

10

1.47

Eastport, Idaho

$2 \quad 3.51$

O N/A

- N/A

2

3.51

CASCADE NATURAL GAS CORPORATION ERI Energy Supply \& Trading

Cascade Natural Gas

Sumas, Washington

$25 \quad 3.51$

160

2.20

152

1.06

337

1.78

CASCADE NATURAL GAS CORPORATION IGI Resources

Cascade Natural Gas

Sumas, Washington

o N/A

$1 \quad 2.56$

O N/A

1

2.56

CASCADE NATURAL GAS CORPORATION

Inland Pacific Energy Services Ltd.

Cascade Natural Gas

Sumas, Washington

150

4.18

137

2.40

152

1.05

439

2.54

CASCADE NATURAL GAS CORPORATION

LG\&E Natural Marketing Canada Inc.

Cascade Natural Gas

Sumas, Washington

293

4.17

305

2.53

O N/A

598

3.34

CASCADE NATURAL GAS CORPORATION PanEnergy Marketing Canada

Cascade Natural Gas

Sumas, Washington

$152 \quad 4.19$

137

2.41

146

1.09

435

2.59

CASCADE NATURAL GAS CORPORATION

Questar Energy

Cascade Natural Gas

Sumas, Washington

$304 \quad 4.26$

o N/A

152

1.39

456

3.30

CASCADE NATURAL GAS CORPORATION

TransCanada Gas Services Limited

Cascade Natural Gas

Sumas, Washington

$3 \quad 3.45$

O N/A

$0 \quad$ N/A

3

3.45

CASCADE NATURAL GAS CORPORATION U.S. Gas Transportation Inc.

Cascade Natural Gas

Sumas, Washington

- N/A

152

2.28

15

1.06

$304 \quad 1.67$

CASCADE NATURAL GAS CORPORATION Westcoast Energy Services

Cascade Natural Gas
Eastport, Idaho o. N/A

0

N/A

8

1.34 
SHORT-TERM SALES

YEAR: 1997 Quarter: One

Estimated Volumes (MMCF) \& Prices (\$ / MMBTU)
Importer

Seller

Purchaser / End User

CASCADE NATURAL GAS CORPORATION

Westcoast Energy Services

Cascade Natural Gas

CASCADE NATURAL GAS CORPORATION Westcoast Gas Services Inc.

Cascade Natural Gas

Eastport, Idaho

CASCADE NATURAL GAS CORPORATION

Westcoast Gas Services inc.

Cascade Natural Gas

CHEVRON U.S.A. INC.

Chevron Canada Resources

Georgia Pacific Corporation

CHEVRON U.S.A. INC.

Chevron Canada Resources

Natural Gas Clearinghouse

CIBOLA CORPORATION

Cibola Canada Energy Marketing Co.

Various IA State Markets

CNG ENERGY SERVICES CORPORATION

TransCanada Pipelines Limited

CNG Energy Services

Waddington, NY

$1160 \quad 2.68$

1057

2.06

$852 \quad 1.22$

3068

2.06

COASTAL GAS MARKETING COMPANY Archer Resources

Various CA State Markets

Eastport, Idaho

162

3.28

138

3.72

$143 \quad 2.45$

443

3.15

COASTAL GAS MARKETING COMPANY B.C. Gas Inc.

Various WA State Markets

Sumas, Washington

$5 \quad 3.12$

$60 \quad 2.18$

$8 \quad 1.19$

73

2.14

COASTAL GAS MARKETING COMPANY Barrington Petroleum Limited

Various CA State Markets

Eastport, Idaho

$142 \quad 3.72$

157

2.44

464

3.13

COASTAL GAS MARKETING COMPANY

CanWest Gas Supply Inc.

Various WA State Market
Sumas, Washington
$133 \quad 2.90$

o N/A

276

3.57
Avg.

Vol.

Price

Quarteriy 


\section{SHORT-TERM SALES \\ YEAR: 1997 Quarter: One \\ Estimated Volumes (MMCF) \& Prices (\$/MMBTU)}

Importer

Seller

Purchaser / End User

COASTAL GAS MARKETING COMPANY

Coastal Gas Marketing, Canada

Various MI State Markets

COASTAL GAS MARKETING COMPANY Enron Capital \& Trade Resources Can

Various WA State Markets

COASTAL GAS MARKETING COMPANY Humble Petroleum Marketing Ltd.

Various CA State Markets

Eastport, Idaho

$659 \quad 1.84$

$569 \quad 1.47$

629

1.76

1858

1.70

COASTAL GAS MARKETING COMPANY

Husky Gas Marketing Inc.

Various MI State Markets

COASTAL GAS MARKETING COMPANY Jordan Petroleum Limited

Various NY State Markets

COASTAL GAS MARKETING COMPANY Morgan Hydrocarbons

Various CA State Markets

Eastport, Idaho

$165 \quad 3.28$

142

3.72

$157 \quad 2.43$

464

3.13

COASTAL GAS MARKETING COMPANY Morgan Hydrocarbons

Various NY State Markets

Niagara Falls, NY

$612 \quad 3.69$

$567 \quad 4.20$

$593 \quad 2.53$

1773

3.47

COASTAL GAS MARKETING COMPANY Noram Energy Services

Various WA State Markets

Sumas, Washington

o N/A

$6 \quad 2.18$

$0 \quad$ N/A

6

2.18

COASTAL GAS MARKETING COMPANY Orbit Oil and Gas Lid.

Various NY State Markets

Niagara Falls, NY

$229 \quad 4.00$

208

4.64

209

2.52

646

3.73

COASTAL GAS MARKETING COMPANY PEMEX

Coastal Gas Marketing Company

Hidalgo, Texas

$238 \quad 3.24$

$263 \quad 2.30$

924

1.74

1425

2.09

COASTAL GAS MARKETING COMPANY

PanCanadian Petroleum Ltd.

Various MI State Markets 
SHORT-TERM SALES

YEAR: 1997 Quarter: One

Estimated Volumes (MMCF) \& Prices (\$ / MMBTU)
Importer

Seller

Purchaser / End User

COASTAL GAS MARKETING COMPANY Petro-Canada Hydrocarbons inc.

Various WA State Markets

COASTAL GAS MARKETING COMPANY Petro-Canada Resources

Various WA State Markets

COASTAL GAS MARKETING COMPANY

Poco Petroleum Ltd.

Various MI State Markets

Noyes, Minnesota

- N/A

12

2.66

$7 \quad 1.62$

19

2.27

COASTAL GAS MARKETING COMPANY

Poco Petroleum Ltd.

Various WA State Markets

Sumas, Washington

$37 \quad 4.16$

21

1.72

$49 \quad 1.23$

107

2.35

COASTAL GAS MARKETING COMPANY ProGas Limited

Various MI State Markets

Noyes, Minnesota

316

3.67

282

2.64

$293 \quad 1.55$

892

2.65

COASTAL GAS MARKETING COMPANY Rigel Oil \& Gas Ltd.

Vanous NY State Markets

Grand Island, NY

- N/A

- N/A

108

2.59

108

2.59

COASTAL GAS MAAKETING COMPANY Rigel Oil \& Gas Lid.

Various NY State Markets

Niagara Falls, NY

174

3.54

157

4.19

164

2.51

494

3.40

COASTAL GAS MARKETING COMPANY Shell Canada Limited

Various MI State Markets

Noyes, Minnesota

- N/A

$6 \quad 2.50$

o. N/A

6

2.50

COASTAL GAS MARKETING COMPANY Tarragon Oil and Gas Limited

Various NY State Markets

Grand Island, NY

O N/A

- N/A

$2 \quad 2.58$

2

2.58

COASTAL GAS MARKETING COMPANY Tarragon Oil and Gas Limited

Various NY State Markets

Niagara Falls, NY

148

134

4.20

139

2.41

420

3.37

COASTAL GAS MARKETING COMPANY

Tenaska Marketing Canada

Various WA State Markets
Sumas, Washington
804

2.39

397

1.92

1200

2.23 


\section{SHORT-TERM SALES \\ YEAR: 1997 Quarter: One \\ Estimated Volumes (MMCF) \& Prices (\$ / MMBTU)}

Importer

Seller

Purchaser / End User

COASTAL GAS MARKETING COMPANY U.S. Gas Transportation Inc.

Various WA State Markets

COASTAL GAS MARKETING COMPANY

Ulster Petroleums Limited

Various CA State Markets

COASTAL GAS MARKETING COMPANY

Uister Petroleums Limited

Various NY State Markets

COASTAL GAS MARKETING COMPANY

Ulster Petroleums Limited

Various NY State Markets

COASTAL GAS MARKETING COMPANY Wainoco Oil Corporation

Various NY State Markets

COASTAL GAS MARKETING COMPANY

Westcoast Gas Services inc.

Various WA State Markets

COENERGY TRADING COMPANY

CoEnergy Trading Company

CNG Energy Services

COENERGY TRADING COMPANY CoEnergy Trading Company

Consolidated Edison

COENERGY TRADING COMPANY CoEnergy Trading Company KCS Energy Management

COENERGY TRADING COMPANY

CoEnergy Trading Company

New England Power Company

COENERGY TRADING COMPANY

CoEnergy Trading Company

Northeast Utilities
Niagara Falis, NY

- N/A

o N/A

42

2.18

42

2.18

Niagara Falls, NY

O N/A

o N/A

50

2.18

50

2.18

Point

Eastport, Idaho

Grand Island, NY

Niagara Falls, NY

Niagara Falls, NY

Waddington, NY

o N/A

- N/A

620

2.18

620

2.18
Niagara Falls, NY
- N/A

- N/A

14

2.18

14

2.18 
SHORT-TERM SALES

YEAR: 1997 Quarter: One

Estimated Volumes (MMCF) \& Prices (\$ / MMBTU)
Importer

Seller

Purchaser/End User

COENERGY TRADING COMPANY CoEnergy Trading Company

Ocean State Power

COENERGY TRADING COMPANY CoEnergy Trading Company

Williams Energy Company

COENERGY TRADING COMPANY Coastal Gas Marketing, Canada Consolidated Edison

COENERGY TRADING COMPANY Coastal Gas Marketing, Canada Ocean State Power

Niagara Falls, NY

$0 \quad$ N/A

$3 \quad 2.60$

o N/A

3

2.60

COENERGY TRADING COMPANY Coastal Gas Marketing, Canada Stand Energy

Niagara Falls, NY

3.93

- N/A

31

2.18

62

3.06

COENERGY TRADING COMPANY El Paso Energy Markeing Canada Consolidated Edison

Niagara Falls, NY

$10 \quad 3.93$

$0 \quad$ N/A

$0 \quad$ N/A

10

3.93

COENERGY TRADING COMPANY El Paso Energy Markeing Canada Phibro Division of Salmon

Niagara Falls, NY

O N/A

- N/A

13

2.18

13

2.18

COENERGY TRADING COMPANY Energy Source Canada, Inc.

Connecticut Natural Gas

Niagara Falls, NY

465

3.93

420

2.60

465

2.18

1350

2.91

COENERGY TRADING COMPANY Enron Gas Marketing Canada CNG Energy Services

Niagara Falls, NY

O N/A

$5 \quad 2.60$

$5 \quad 2.18$

10

2.39

COENERGY TRADING COMPANY Enron Gas Marketing Canada

Consolidated Edison

Niagara Falls, NY

o. N/A

50

2.60

36

2.18

86

2.42

COENERGY TRADING COMPANY Enron Gas Marketing Canada New England Power Company
Waddington, NY

280

2.60

$0 \quad$ N/A

590

3.30 


\section{SHORT-TERM SALES \\ YEAR: 1997 Quarter: One \\ Estimated Volumes (MMCF) \& Prices (\$/MMBTU)}

Importer

Seller

Purchaser / End User

COENERGY TRADING COMPANY

Enron Gas Marketing Canada

Northeast Utilities

Niagara Falls, NY

COENERGY TRADING COMPANY Enron Gas Marketing Canada Northeast Utilities

COENERGY TRADING COMPANY Enron Gas Marketing Canada

Phibro Division of Salmon

COENERGY TRADING COMPANY Enron Gas Marketing Canada Williams Energy Company

COENERGY TRADING COMPANY Midcon Gas Services Corporation

Phibro Division of Salmon

Niagara Falls, NY

$6 \quad 3.93$

$0 \quad$ N/A

$0 \quad$ N/A

6 3.93

Niagara Falls, NY

$0 \quad$ N/A

$0 \quad$ N/A

23

2.18

23

COENERGY TRADING COMPANY National Gas \& Electric

Ocean State Power

Niagara Falls, NY

- N/A

$0 \quad$ N/A

10

2.18

10

2.18

COENERGY TRADING COMPANY

Ocean State Power

Consolidated Edison

COENERGY TRADING COMPANY Ocean State Power

Williams Energy Company

COENERGY TRADING COMPANY Renaissance Energy Ltd.

CNG Energy Services

COENERGY TRADING COMPANY

Renaissance Energy Ltd.

Connecticut Natural Gas
Niagara Falls; NY

Niagara Falls, NY

Niagara Falls, NY

$10 \quad 3.93$

$5 \quad 2.60$

O N/A

15

3.49

$30 \quad 2.18$

$149 \quad 2.18$
O N/A

140

2.60 $\begin{array}{llll}0 & \text { N/A } & 140 \quad 2.60\end{array}$

Quarterly
Total
Avg.
Vol. Price

$5 \quad 2.60$

$5 \quad 2.60$

$20 \quad 2.18$

$0 \quad N / A$


SHORT-TERM SALES

YEAR: 1997 Quarter: One

Estimated Volumes (MMCF) \& Prices (\$ / MMBTU)

Importer

Seller

Purchaser / End User

COENERGY TRADING COMPANY Renaissance Energy Ltd.

Consolidated Edison

COENERGY TRADING COMPANY Renaissance Energy Ltd.

Empire Natural Gas Corporation

COENERGY TRADING COMPANY

Renaissance Energy Ltd.

New England Power Company

COENERGY TRADING COMPANY Renaissance Energy Ltd.

Pawtucket Power Associates

Niagara Falls, NY

$11 \quad 3.93$

- N/A

$18 \quad 3.93$

O N/A

- N/A

18

3.93

COENERGY TRADING COMPANY Renaissance Energy Ltd.

Pittsfield Generating

COENERGY TRADING COMPANY Renaissance Energy Ltd.

Stand Energy

Niagara Falls, NY

o N/A

$5 \quad 2.60$

$0 \quad$ N/A

5

2.60

COENERGY TRADING COMPANY Stampeder Energy

Pittsfield Generating

COENERGY TRADING COMPANY TransCanada Gas Services Limited CNG Energy Services

COENERGY TRADING COMPANY TransCanada Gas Services Limited Consolidated Edison

Niagara Falls, NY

101

2.60

o N/A

123

2.84

COENERGY TRADING COMPANY TransCanada Gas Services Limited KCS Energy Marketing
$156 \quad 3.93$

O N/A

- N/A

156

3.93
Niagara Falls, NY 
Importer

Seller

Purchaser / End User

COENERGY TRADING COMPANY

TransCanada Gas Services Limited Northeast Utilities

COENERGY TRADING COMPANY TransCanada Gas Services Limited Northeast Utilities

COENERGY TRADING COMPANY TransCanada Gas Services Limited Ocean State Power

COENERGY TRADING COMPANY TransCanada Gas Services Limited Phibro Division of Salmon

Niagara Falls, NY

$6 \quad 3.93$

$4 \quad 2.60$

- N/A

10

3.35

COENERGY TRADING COMPANY TransCanada Gas Services Limited Wascana Energy Marketing

Niagara Falls, NY

$0 \quad$ N/A

141

2.60

O N/A

141

2.60

COENERGY TRADING COMPANY Wascana Energy Marketing Inc.

CNG Energy Services

COENERGY TRADING COMPANY Wascana Energy Marketing Inc.

Phibro Division of Salmon

COENERGY TRADING COMPANY Wickford Energy

CNG Energy Services

Niagara Falls, NY

$3 \quad 3.93$

$0 \quad$ N/A

O N/A

3

3.93

COENERGY TRADING COMPANY Wickford Energy

Consolidated Edison

Niagara Falls, NY

$16 \quad 3.93$

20

2.60

$0 \quad N / A$

36

3.19

COENERGY TRADING COMPANY Wickford Energy

Phibro Division of Salmon

Niagara Falls, NY

13.93

O N/A

$0 \quad$ N/A

1

3.93

COENERGY TRADING COMPANY Wickford Energy

Phoenix Soil

Niagara Falls, NY
- N/A

$6 \quad 2.60$

o N/A

$6 \quad 2.60$ 
SHORT-TERM SALES

YEAR: 1997 Quarter: One

Estimated Volumes (MMCF) \& Prices (\$ / MMBTU)
Importer

Seller

Purchaser / End User

COENERGY TRADING COMPANY

Williams Energy Services Company

CNG Energy Services

COENERGY TRADING COMPANY Williams Energy Services Company Ocean State Power

CONOCO INC

PEMEX

Conoco Inc.

CONSUMERS ENERGY COMPANY

TransCanada Gas Services Limited

Consumers Power Company

Noyes, Minnesota

$465 \quad 1.97$

420

1.97

465

1.97

1350

1.97

CORAL ENERGY RESOURCES, L.P.

PEMEX

Various TX State Markets

Hidalgo, Texas

191

2.63

139

2.73

$0 \quad$ N/A

330

2.67

CORAL ENERGY RESOURCES, L.P.

Shell Canada Limited

Michigan Consolidated Gas Company

Noyes, Minnesota

460

$414 \quad 2.57$

$460 \quad 2.06$

1334

2.87

CORPUS CHRISTI GAS MARKETING, L.P. PEMEX

Corpus Christi Gas Marketing, L.P.

Hidalgo, Texas

96

3.15

$43 \quad 1.81$

$15 \quad 2.30$

154

2.70

COWEST ENERGY

Cowest Energy

Cowest Energy

DARTMOUTH POWER ASSOCIATES L.P.

Dartmouth Power Associates

AIG Trading Corporation

Waddington, NY

$405 \quad 3.52$

$0 \quad$ N/A

- N/A

405

3.52

DARTMOUTH POWER ASSOCIATES L.P.

Dartmouth Power Associates

CNG Energy Services

Waddington, NY

$5 \quad 2.37$

$5 \quad 1.57$

$0 \quad$ N/A

$10 \quad 1.96$

DARTMOUTH POWER ASSOCIATES L.P.

Dartmouth Power Associates

CoEnergy Trading Company

Waddington, NY
- N/A

$7 \quad 1.58$

o N/A

$7 \quad 1.58$ 
SHORT-TERM SALES

YEAR: 1997 Quarter: One

Estimated Volumes (MMCF) \& Prices (\$ / MMBTU)

Importer

Seller

Purchaser / End User

DARTMOUTH POWER ASSOCIATES L.P.

Dartmouth Power Associates

Yankee Gas Services

Waddington, NY

$28 \quad 2.59$

0. N/A

O N/A

28

2.59

\section{DEK ENERGY COMPANY}

Apache Canada Ltd.

National Gas \& Electric

DISTRIGAS CORPORATION

Abu Dhabi Gas Liquefaction Co., Ltd

American National Power Co.

DISTRIGAS CORPORATION

Abu Dhabi Gas Liquefaction Co., Ltd Aquila Energy Marketing

DISTRIGAS CORPORATION

Abu Dhabi Gas Liquefaction Co., Ltd Bay State Gas Company

DISTRIGAS CORPORATION

Abu Dhabi Gas Liquefaction Co., Ltd Beacon Management

Everett, MA

$6 \quad 3.35$

$0 \quad$ N/A

$0 \quad$ N/A

6

3.35

DISTRIGAS CORPORATION

Abu Dhabi Gas Liquefaction Co., Ltd Berkshire Gas Company

Everett, MA

$66 \quad 3.35$

o N/A

o N/A

66

3.35

DISTRIGAS CORPORATION

Abu Dhabi Gas Liquefaction Co., Ltd Boston Edison Company

Everett, MA

$26 \quad 3.35$

o N/A

o N/A

26

3.35

DISTRIGAS CORPORATION

Abu Dhabi Gas Liquefaction Co., Ltd Boston Gas Company

Everett, MA

$334 \quad 3.35$

O N/A

O N/A

334

3.35

DISTRIGAS CORPORATION

Abu Dhabi Gas Liquefaction Co., Ltd

CNG Gas Services

Everett, MA

$10 \quad 3.35$

$0 \quad N / A$

$0 \quad N / A$

10

3.35

DISTRIGAS CORPORATION

Abu Dhabi Gas Liquefaction Co., Ltd CoEnergy Trading Company
Everett, MA

$50 \quad 3.35$

- N/A

O N/A

50

3.35 
SHORT-TERM SALES

YEAR: 1997 Quarter: One

Estimated Volumes (MMCF) \& Prices (\$ / MMBTU)
Importer

Seller

Purchaser/End User

DISTRIGAS CORPORATION

Abu Dhabi Gas Liquefaction Co., Ltd

Coastal Gas. Marketing Company

DISTRIGAS CORPORATION

Abu Dhabi Gas Liquefaction Co., Ltd

Colonial Gas Company

DISTRIGAS CORPORATION

Abu Dhabi Gas Liquefaction Co., Ltd Columbia Bay Towers

Point

Entry

Everett, MA

Everett, MA

Everett, MA

Everett, MA

Everett, MA

Abu Dhabi Gas Liquefaction Co., Ltd

Connecticut Natural Gas

DISTRIGAS CORPORATION

Abu Dhabi Gas Liquefaction Co., Ltd

Eastem Energy Marketing

DISTRIGAS CORPORATION

Abu Dhabi Gas Liquefaction Co., Ltd El Paso Gas Marketing

DISTRIGAS CORPORATION

Abu Dhabi Gas Liquefaction Co., Ltd Energynorth inc.

DISTRIGAS CORPORATION

Abu Dhabi Gas Liquefaction Co., Ltd Enron Gas Marketing

Everett, MA

$14 \quad 3.35$

O N/A

O N/A

14

3.35

DISTRIGAS CORPORATION Abu Dhabi Gas Liquefaction Co., Ltd Essex County Gas Company

DISTRIGAS CORPORATION

Abu Dhabi Gas Liquefaction Co., Ltd Fall River Gas Company

January

Vol. Price

February

Vol. Price

March

Vol. Price

- N/A

10

3.35

$132 \quad 3.35$

- N/A

- N/A

132

3.35

Everett, MA

$12 \quad 3.35$

$0 \quad N / A$

$0 \quad$ N/A

12

Everett, MA

$141 \quad 3.35$

$0 \quad$ N/A

$0 \quad$ N/A

141

3.35

Everett, MA

48. $\quad 3.35$

- N/A

- N/A

48

3.35

Everett, MA

$132 \quad 3.35$ o N/A

N/A

132

3.35 
SHORT-TERM SALES

YEAR: 1997 Quarter: One

Estimated Volumes (MMCF) \& Prices (\$ / MMBTU)
Importer

Seller

Purchaser/End User

DISTRIGAS CORPORATION

Abu Dhabi Gas Liquefaction Co., Ltd

Fitchburg Gas \& Electric Company

DISTRIGAS CORPORATION

Abu Dhabi Gas Liquefaction Co., Ltd Gasmark Inc.

DISTRIGAS CORPORATION

Abu Dhabi Gas Liquefaction Co., Ltd Global Petroleum

Point

Entry

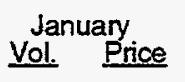

February

Vol. Price

March

Vol. Price

Quarterly

Avg.

Vol. Prg. Price

Everett, MA

$9 \quad 3.35$

- N/A

o N/A

9

3.35

Everett, MA

$2 \quad 3.35$

- N/A

- N/A

$2 \quad 3.35$

Everett, MA

$3 \quad 3.35$

- N/A

o N/A

$3 \quad 3.35$

Everett, MA

$24 \quad 3.35$

- N/A

- N/A

24

Holyoke Gas Company

DISTRIGAS CORPORATION

Abu Dhabi Gas Liquefaction Co., Ltd Industrial Heat Treating Company

Everett, MA

$1 \quad 3.35$

$0 \quad$ N/A

- N/A

1

3.35

DISTRIGAS CORPORATION

Abu Dhabi Gas Liquefaction Co., Ltd L'Energia L.P.

Everett, MA

$137 \quad 3.35$

- N/A

o N/A

137

3.35

DISTRIGAS CORPORATION

Abu Dhabi Gas Liquefaction Co., Ltd L.E. Mason Company

Everett, MA

$2 \quad 3.35$

- N/A

- N/A

2

3.35

DISTRIGAS CORPORATION

Abu Dhabi Gas Liquefaction Co., Ltd Louis Dreyfus Energy Corporation

DISTRIGAS CORPORATION

Abu Dhabi Gas Liquefaction Co., Ltd Masspower

Everett, MA

$11 \quad 3.35$

o N/A

- N/A

11

3.35

DISTRIGAS CORPORATION

Abu Dhabi Gas Liquefaction Co., Lid Middleborough Gas Company

Everett, MA

$12 \quad 3.35$

- N/A

- N/A

12

3.35

DISTRIGAS CORPORATION

Abu Dhabi Gas Liquefaction Co., Lid Natural Gas Clearinghouse

Everett, MA

$96 \quad 3.35$

- N/A

o N/A

96

3.35 
SHORT-TERM SALES

YEAR: 1997 Quarter: One

Estimated Volumes (MMCF) \& Prices (\$ / MMBTU)
Importer

Seller

Purchaser / End User

DISTRIGAS CORPORATION

Abu Dhabi Gas Liquefaction Co., Lto New England Power Company

DISTRIGAS CORPORATION

Abu Dhabi Gas Liquefaction Co., Ltd New Jersey Natural Gas

Everett, MA

DISTRIGAS CORPORATION

Abu Dhabi Gas Liquefaction Co., Ltd North Attleboro Gas Company

DISTRIGAS CORPORATION

Abu Dhabi Gas Liquefaction Co., Ltd Northem Utilities, Inc.

Everett, MA

40

3.35

$0 \quad$ N/A

$0 \quad$ N/A

40

3.35

DISTRIGAS CORPORATION

Abu Dhabi Gas Liquefaction Co., Ltd

PanEnergy Trading \& Marketing Svcs.

DISTRIGAS CORPORATION

Abu Dhabi Gas Liquefaction Co., Ltd

South Jersey Gas Company

DISTRIGAS CORPORATION

Abu Dhabi Gas Liquefaction Co., Ltd

Southem Connecticut Gas Company

DISTRIGAS CORPORATION

Abu Dhabi Gas Liquefaction Co., Lid Supreme Energy Company

DISTRIGAS CORPORATION

Abu Dhabi Gas Liquefaction Co., Ltd

Valley Gas Company

Everett, MA

$64 \quad 3.35$

- N/A

O N/A

64

3.35

Everett, MA

$14 \quad 3.35$

$0 \quad$ N/A

- N/A

$14 \quad 3.35$

Everett, MA

30

3.35

o. N/A

- N/A

30

3.35

Everett, MA

$1 \quad 3.35$

o N/A

O N/A

$1 \quad 3.35$

DISTRIGAS CORPORATION

Abu Dhabi Gas Liquefaction Co., Ltd Winchester Hospital

Everett, MA

$2 \quad 3.35$

O N/A

$0 \quad$ N/A

$2 \quad 3.35$

DISTRIGAS CORPORATION

Abu Dhabi Gas Liquefaction Co., Ltd Wollaston Alloys, inc.
Everett, MA

$1 \quad 3.35$

- N/A

- N/A

1

3.35 
SHORT-TERM SALES

YEAR: 1997 Quarter: One

Estimated Volumes (MMCF) \& Prices (\$ / MMBTU)
Importer

Seller

Purchaser / End User

DISTRIGAS CORPORATION

Abu Dhabi Gas Liquefaction Co., Ltd

$X$ Energy, Inc.

EASTERN ENERGY MARKETING, INC.

Renaissance Energy Ltd.

Various NY State Markets

Grand Island, NY

Grand Island, NY

Westcoast Gas Services inc.

Various NY State Markets

Point

Ef

Everett, MA

$21 \quad 3.35$

$0 \quad$ N/A

O N/A

21

3.35

EL PASO ENERGY MARKETING COMPANY

El Paso Energy Markeing Canada

Various NY State Markets

Niagara Falls, NY

83

4.12

0

N/A

- N/A

83

4.12

EL PASO ENERGY MARKETING COMPANY

PEMEX

Various TX State Markets

Hidalgo, Texas

$10 \quad 2.20$

151

2.72

$3 \quad 1.59$

164

2.66

ENRON CAPITAL \& TRADE RESOURCES CORP.

B.C. Gas Inc.

Various CA State Markets Eastport, Idaho

$114 \quad 1.51$

202

1.23

386

1.51

ENRON CAPITAL \& TRADE RESOURCES CORP.

B.C. Gas Inc.

Various CA State Markets

Sumas, Washington

116

3.13

1.67

143

1.19

312

1.99

ENRON CAPITAL \& TRADE RESOURCES CORP.

Cook Inlet Energy Supply

Various CA State Markets

Eastport, Idaho

O N/A

$10 \quad 1.35$

$22 \quad 1.27$

32

1.29

ENRON CAPITAL \& TRADE RESOURCES CORP.

Cook Inlet Energy Supply

Various CA State Markets

Sumas, Washington

ENRON CAPITAL \& TRADE RESOURCES CORP.

Enron Capital \& Trade Resources Can

Vanous CA State Markets

Eastport, Idaho

350

1.72

$0 \quad$ N/A

411

1.90

ENRON CAPITAL \& TRADE RESOURCES CORP.

Enron Capital \& Trade Resources Can

Various CA State Markets

Sumas, Washington
$991 \quad 3.02$

895

1.94

804

1.13

2690

2.10 
SHORT-TERM SALES

YEAR: 1997 Quarter: One

Estimated Volumes (MMCF) \& Prices (\$ / MMBTU)

Importer

Seller

Purchaser / End User

Point

Ef

ENRON CAPITAL \& TRADE RESOURCES CORP.

Enron Capital \& Trade Resources Can

Various MI State Markets

Detroit, Michigan

ENRON CAPITAL \& TRADE RESOURCES CORP.

Enron Capital \& Trade Resources Can

Various MI State Markets

St. Clair, Michigan

ENRON CAPITAL \& TRADE RESOURCES CORP.

Enron Capital \& Trade Resources Can

Various NY State Markets

Grand Island, NY

ENRON CAPITAL \& TRADE RESOURCES CORP

Enron Capital \& Trade Resources Can

Various NY State Markets

Waddington, NY

ENRON CAPITAL \& TRADE RESOURCES CORP

PEMEX

Various TX State Markets

Hidalgo, Texas

$65 \quad 3.15$

Grand Island, NY

$0 \quad$ N/A

o N/A

$1 \quad 2.15$

1

2.15

Colonial Energy
Various NY State Markets

ENSERCH GAS COMPANY

EES Canada

Various CA State Markets

Eastport, Idaho

$14 \quad 4.21$

$147 \quad 2.04$

D N/A

160

2.23

ENSERCH GAS COMPANY

Energy Source Canada, Inc.

Various NY State Markets

Grand Island, NY

$310 \quad 5.30$

140

3.77

155

2.72

605

4.28

ENSERCH GAS COMPANY

LG\&E Natural Marketing Canada Inc.

Various NY State Markets

Grand Island, NY

$0 \quad N / A$

$0 \quad$ N/A

$2 \quad 1.97$

$2 \quad 1.97$

ENSERCH GAS COMPANY

Louis Dreyfus Energy Corporation

Various NY State Markets

Grand Island, NY

111

5.11

78

3.66

- N/A

189

4.51

ENSERCH GAS COMPANY

N.Y. State Electric \& Gas

Various NY State Markets
Grand Island, NY
- N/A

74

2.00

74

2.00
Avg

Vol. Price

$41 \quad 3.59$

3.68

2.75

2.04

Quarterly 


\section{SHORT-TERM SALES \\ YEAR: 1997 Quarter: One \\ Estimated Volumes (MMCF) \& Prices (\$/MMBTU)}

Importer

Seller

Purchaser / End User

ENSERCH GAS COMPANY

Renaissance Energy Ltd.

Various NY State Markets

ENSERCH GAS COMPANY

Semco Energy Services, Inc.

Various NY State Markets

ENSERCH GAS COMPANY

Temco

Various NY State Markets

ENSERCH GAS COMPANY

Temoo

Various NY State Markets

ENSERCH GAS COMPANY

TransCanada Gas Services Limited

Various NY State Markets

Grand Island, NY

ENSERCH GAS COMPANY

TransCanada Gas Services Limited

Various NY State Markets

ENSERCH GAS COMPANY

Wascana Energy Marketing Inc.

Various NY State Markets

ENSERCH GAS COMPANY

Wascana Energy Marketing Inc.

Various NY State Markets

ENSERCH GAS COMPANY

Westcoast Gas Services Inc.

Various NY State Markets

Grand Island, NY

$93 \quad 5.07$

84

3.69

$93 \quad 2.70$

270

3.82

ENSERCH GAS COMPANY

Williams Energy Services Company

Various NY State Markets

January

February
Vol. Price

March

Vol. Price

$7 \quad 2.26$

7

2.26

Grand Island, NY

310

280

3.09

310

2.33

900

3.40

Grand Island, NY

$\begin{array}{llll}48 & 3.20 & 116 & 3.35\end{array}$

$23 \quad 2.23$

186

3.18

Niagara Falls, NY

Niagara Falls, NY

Grand Island, NY

Niagara Falls, NY

Grand Island, NY

O N/A

$5 \quad 3.25$

$0 \quad$ N/A

$5 \quad 3.25$

EQUITABLE RESOURCES MARKETING COMPANY

Equitable Resources Marketing Canad

Equitable Resources Marketing Co.

Eastport, Idaho 
SHORT-TERM SALES

YEAR: 1997 Quarter: One

Estimated Volumes (MMCF) \& Prices (\$ / MMBTU)

Importer

Seller

Purchaser / End User

EQUITABLE RESOURCES MARKETING COMPANY

Equitable Resources Marketing Canad

Equitable Resources Marketing Co.

GREAT FALLS GAS COMPANY (SP)

Eagle Gas Marketing

Vanous MT State Markets

Point

of

Entry

Sumas, Washington

Babb, Montana

529

2.19

302

1.47

$102 \quad 1.62$

92

1.39

31

1.17

224

1.46

HOWARD ENERGY COMPANY, INC.

AIG Trading Corporation

Howard Energy Company

Grand Island, NY

$3 \quad 3.20$

$0 \quad$ N/A

O N/A

3

3.20

HOWARD ENERGY COMPANY, INC.

AIG Trading Corporation

Howard Energy Company

Niagara Falls, NY

3.20

$0 \quad$ N/A

- N/A

32

3.20

HOWARD ENERGY COMPANY, INC.

SEMCO

Howard Energy Company

Grand Island, NY

O N/A

$84 \quad 3.64$

93

2.43

177

3.00

HOWARD ENERGY COMPANY, INC.

TransCanada Gas Services Limited

Howard Energy Company

Grand Island, NY

- N/A

33

3.40

- N/A

33

3.40

HOWARD ENERGY COMPANY, INC.

Wascana Energy Marketing Inc.

Howard Energy Company

Grand Island, NY

$46 \quad 3.65$

$2 \quad 3.27$

o N/A

47

3.63

HUSKY GAS MARKETING, INC.

Husky Oil

Cibola Corporation

Port of Morgan, MT

195

3.37

230

2.12

15

1.25

581

2.30

HUSKY GAS MARKETING, INC.

Husky Oil
Coastal Gas Marketing Company

HUSKY GAS MARKETING, INC.

Husky Oil

Enron Capital \& Trade Resources

Sumas, Washington

306

4.17

277

2.79

306

1.80

889

2.92

SP Interruptible/special purchase sale made under Long-term authorization.

$\begin{array}{llllllll}0 & \text { N/A } & 3 & \text { N/A } & 0 & \text { N/A } & 3 & 0.00\end{array}$


SHORT-TERM SALES

YEAR: 1997 Quarter: One

Estimated Volumes (MMCF) \& Prices (\$ / MMBTU)
Importer

Seller

Purchaser / End User

HUSKY GAS MARKETING, INC. Husky Oil

IGI Resources

HUSKY GAS MARKETING, INC.

Husky Oil

Interenergy Corporation

HUSKY GAS MARKETING, INC.

Husky Oil

Koch Gas Services Company

HUSKY GAS MARKETING, INC.

Husky Oil

Mock Resources

HUSKY GAS MARKETING, INC.

Husky Oil

Natural Gas Transmission

Sumas, Washington

$11 \quad 1.18$

$26 \quad 2.05$

$63 \quad 1.13$

$100 \quad 1.37$

HUSKY GAS MARKETING, INC

Husky Oil

Omaha Utilities District

Port of Morgan, MT

158

3.37

142

2.12

145

March
Vol. Price

Quarterty

Avg.

Vol. Price

HUSKY GAS MARKETING, INC.

Husky Oil

Peoples Gas, Light, \& Coke

Port of Morgan, MT

306

3.37

284

2.12

31

O N/A

$48 \quad 1.18$

HUSKY GAS MARKETING, INC.

Husky Oil

Phoenix Chemical

Port of Morgan, MT

155

3.37

140

2.12

$154 \quad 1.25$

448

2.25

HUSKY GAS MARKETING, INC. Husky Oil

Southwest Gas Corporation

Sumas, Washington

$11 \quad 1.18$

77 N/A

- N/A

88

0.15

HUSKY GAS MARKETING, INC.

Husky Oil

Tenaska Gas Company

Port of Morgan, MT

3.37

175

2.12

$96 \quad 1.25$

447

2.43

HUSKY GAS MARKETING, INC. Husky Oil

Terra International
Port of Morgan, MT
316

3.37

285

2.12

274 


\section{SHORT-TERM SALES \\ YEAR: 1997 Quarter: One \\ Estimated Volumes (MMCF) \& Prices (\$ / MMBTU)}

Importer

Seller

Purchaser / End User

HUSKY GAS MARKETING, INC.

Husky Oil

U.S. Energy Services

HUSKY GAS MARKETING, INC.

Husky Oil

U.S. Gas Transportation Inc.

HUSKY GAS MARKETING, INC.

Husky Oil

Utilicorp United

Noyes, Minnesota

Port of Morgan, MT

$391 \quad 3.37$

324

2.12

$372 \quad 1.25$

1087

2.27

HUSKY GAS MARKETING, INC.

Husky Oil

Valero Gas Marketing

Port of Morgan, MT

$127 \quad 3.37$

22

2.12

166

1.25

315

2.17

HUSKY GAS MARKETING, INC.

Husky Oil

Washington Natural Gas Company

HUSKY GAS MARKETING, INC.

Husky Oil

Westcoast Gas Services (U.S.A.) Inc

Port of Morgan, MT

$155 \quad 3.37$

208

2.12

$187 \quad 1.25$

550

2.18

HUSKY GAS MARKETING, INC.

Husky Oil

Western Gas Resources

Sumas, Washington

- N/A

O N/A

$4 \quad 1.13$

4

1.13

IGI RESOURCES INC.

Amoco Canada Petroleum Company Ltd. Aluminum Company of America

Eastport, Idaho

$34 \quad 1.67$

$25 \quad 2.11$

$24 \quad 1.30$

83

1.70

IGI RESOURCES INC.

Amoco Canada Petroleum Company Ltd.

American Fine Foods

Eastport, idaho

11.66

$1 \quad 2.11$

$0 \quad$ N/A

$2 \quad 1.87$

IGI RESOURCES INC.

Amoco Canada Petroleum Company Ltd. Barret Fuels
Eastport, Idaho
O N/A

$35 \quad 1.30$

35

1.30 


\section{SHORT-TERM SALES \\ YEAR: 1997 Quarter: One \\ Estimated Volumes (MMCF) \& Prices (\$ / MMBTU)}

Importer

Seller

Purchaser/End User

IGI RESOURCES INC.

Amoco Canada Petroleum Company Ltd. Basic American Foods

IGI RESOURCES INC.

Amoco Canada Petroleum Company Ltd. Boise Cascade Corporation

IGI RESOURCES INC.

Amoco Canada Petroleum Company Ltd. Borden

Eastport, Idaho

IGI RESOURCES INC.

Amoco Canada Petroleum Company Ltd. Bullseye Glass

IGI RESOURCES INC.

Amoco Canada Petroleum Company Ltd. Cascade Natural Gas

Point

of

Eastport, Idaho

Eastport, Idaho

$1 \quad 1.66$

$1 \quad 1.66$

- N/A

21.30

$2 \quad 1.41$

Eastport, Idaho

Eastport, Idaho

$105 \quad 1.67$

81

2.10

79

1.30

266

1.69

IGI RESOURCES INC.

Amoco Canada Petroleum Company Ltd. Chevron U.S.A. Inc.

Eastport, Idaho

IGI RESOURCES INC.

Amoco Canada Petroleum Company Ltd. ConAgra Energy

Eastport, Idaho

IGI RESOURCES INC

Amoco Canada Petroleum Company Ltd. ESCO Comoration

Eastport, Idaho

$2 \quad 1.67$

$1 \quad 2.11$

$1 \quad 1.30$

$4 \quad 1.75$

IGI RESOURCES INC

Amoco Canada Petroleum Company Ltd. Eagle Picher

Eastport, Idaho

$1 \quad 1.66$

$2 \quad 2.11$

$1 \quad 1.30$

3

1.84

IGI RESOURCES INC.

Amoco Canada Petroleum Company Ltd.

Evanite Fiber

Eastport, Idaho

$1 \quad 1.66$

$2 \quad 2.11$

$8 \quad 1.30$

$11 \quad 1.49$

IGI RESOURCES INC

Amoco Canada Petroleum Company Ltd. J.R. Simplot Company
Eastport, Idaho
227

2.10

200

1.30

869

1.70 


\section{SHORT-TERM SALES \\ YEAR: 1997 Quarter: One \\ Estimated Volumes (MMCF) \& Prices (\$ / MMBTU)}

Importer

Seller

Purchaser/End User

IGI RESOURCES INC.

Amoco Canada Petroleum Company Ltd. Jorgenson Forge

IGI RESOURCES INC.

Amoco Canada Petroleum Company Ltd. Lakeside

Eastport, Idaho

Eastport, Idaho

Amoco Canada Petroleum Company Ltd. Lamb Weston

IGI RESOURCES INC.

Amoco Canada Petroleum Company Ltd.

Point
of
Entry

Eastport, Idaho

January
Vol. Price

February

Vol. Price

March

Vol. Price

$\begin{array}{ll}1 & 2.11\end{array}$

O N/A

$1 \quad 1.66$

$2 \quad 2.11$

0. N/A

$1 \quad 1.66$

$1 \quad 2.11$

$0 \quad$ N/A

11.66

$1 \quad 2.11$

$1 \quad 1.30$ Legacy Health System

Eastport, Idaho

Eastport, Idaho

Eastport, Idaho

Amoco Canada Petroleum Company Ltd. NORPAC

IGI RESOURCES INC.

Amoco Canada Petroleum Company Ltd. Oroweat

Eastport, Idaho

[G] RESOURCES INC.

Amoco Canada Petroleum Company Ltd. PITCO

Eastport, Idaho

IGI RESOURCES INC.

Amoco Canada Petroleum Company Ltd. Pacific Gas \& Electric (UEG)

IGI RESOURCES INC.

Amoco Canada Petroleum Company Lid. PanEnergy Trading \& Marketing Svcs.

IGI RESOURCES INC.

Amoco Canada Petroleum Company Ltd. Pendelton

Eastport, Idaho

Eastport, Idaho
- N/A

1

$3 \quad 1.97$

$2 \quad 1.91$

$3 \quad 1.82$

O N/A

$49 \quad 2.10$

$0 \quad$ N/A

49

2.10

0. N/A

$0 \quad$ N/A

$52 \quad 1.30$

52

1.30

O N/A

ON/A

123

1.30

123

1.30

11.66

0

N/A

21.30

$2 \quad 1.39$ 


\section{SHORT-TERM SALES \\ YEAR: 1997 Quarter: One \\ Estimated Volumes (MMCF) \& Prices (\$ / MMBTU)}

Importer

Seller

Purchaser / End User

IGI RESOURCES INC.

Amoco Canada Petroleum Company Ltd. WP Natural Gas

IGI RESOURCES INC.

Amoco Canada Petroleum Company Ltd. Washington Natural Gas Company

IGI RESOURCES INC.

Amoco Canada Petroleum Company Ltd. Washington Water Power Company

IGI RESOURCES INC.

Amoco Canada Petroleum Company Ltd. Weyerhauser

IGI RESOURCES INC.

Amoco Canada Petroleum Company Ltd. Willamette

Eastport, Idaho

Eastport, Idaho

IGI RESOURCES INC.

B.C. Gas Inc.

Aluminum Company of America

IGI RESOURCES INC.

B.C. Gas Inc.

Barret Fuels

Eastport, Idaho

Eastport, Idaho

B.C. Gas Inc.

Basic American Foods

IGI RESOURCES INC.

B.C. Gas Inc.

Boise Cascade Corporation

IGI RESOURCES INC.

B.C. Gas Inc.

Cascade Natural Gas

IGI RESOURCES INC.

B.C. Gas Inc.

Evanite Fiber

Point

Entry

Eastport, Idaho

Eastport, Idaho

Eastport, Idaho

Eastport, Idaho

Eastport, Idaho

Eastport, Idaho
Eastport, Idaho

$53 \quad 1.67$

- N/A

$31 \quad 1.30$

$84 \quad 1.54$

o N/A

$2 \quad 2.11$

- N/A

$2 \quad 2.11$

$4 \quad 1.67$

$2 \quad 2.11$

$4 \quad 1.30$

$10 \quad 1.59$

$6 \quad 1.67$

40

2.11

$50 \quad 1.30$

$96 \quad 1.66$

$114 \quad 1.67$

$39 \quad 2.11$

$40 \quad 1.30$

193

1.68

- N/A

o N/A

$6 \quad 1.18$

$6 \quad 1.18$

- N/A

o. N/A

1

$9 \quad 1.18$

$\begin{array}{ll}9 & 1.18\end{array}$

o N/A

- N/A

o N/A

1

1.18

1

1.18

- N/A

- N/A

20

1.18

20

1.18
- N/A

0

N/A

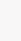

$2 \quad 1.18$

$2 \quad 1.18$ 
SHORT-TERM SALES

YEAR: 1997 Quarter: One

Estimated Volumes (MMCF) \& Prices (\$ / MMBTU)
Importer

Seller

Purchaser/ End User

IGI RESOURCES INC.

B.C. Gas Inc

J.R. Simplot Company

IGI RESOURCES INC.

B.C. Gas Inc.

Pacific Gas \& Electric (UEG)

IGI RESOURCES INC.

B.C. Gas Inc.

PanEnergy Trading \& Marketing Svcs.

IGI RESOURCES INC.

B.C. Gas Inc.

WP Natural Gas

IGI RESOURCES INC.

B.C. Gas Inc.

Washington Water Power Company

Eastport, Idaho

B.C. Gas Inc.

Willamette

IGI RESOURCES INC.

CanWest Gas Supply Inc.

Aluminum Company of America

IGI RESOURCES INC.

CanWest Gas Supply Inc.

Barret Fuels

IGI RESOURCES INC.

CanWest Gas Supply inc.

Cascade Natural Gas

IGI RESOURCES INC.

CanWest Gas Supply inc.

J.R. Simplot Company

Eastport, Idaho

ESINC.

B.C. Gas Ino.
Eastport, Idaho

Eastport, Idaho

Eastport, Idaho

Eastport, Idaho

Point
of

Eastport, Idaho

Eastport, Idaho

Eastport, Idaho
Eastport, Idaho

January
Vol. Price

March
Vol. Price

Quarterly

Total

Avg. ${ }^{\text {Avgice }}$

- N/A

- N/A

$52 \quad 1.18$

52

1.18

- N/A

- N/A

$13 \quad 1.18$

$13 \quad 1.18$

- N/A

- N/A

$13 \quad 1.18$

$13 \quad 1.18$

- N/A

- N/A

$10 \quad 1.18$

$10 \quad 1.18$

$0 \quad$ N/A

$2 \quad 1.09$

$2 \quad 1.09$

o N/A

- N/A

$5 \quad 1.09$

$5 \quad 1.09$

- N/A

$13 \quad 1.09$

13

1.09 


\section{SHORT-TERM SALES \\ YEAR: 1997 Quarter: One \\ Estimated Volumes (MMCF) \& Prices (\$ / MMBTU)}

Importer

Seller

Purchaser / End User

IGI RESOURCES INC.

CanWest Gas Supply inc.

J.R. Simplot Company

IGI RESOURCES INC.

CanWest Gas Supply Inc.

Pacific Gas \& Electric (UEG)

IGI RESOURCES INC.

CanWest Gas Supply inc.

PanEnergy Trading \& Marketing Svcs. Eastport, Idaho

Point

of

Entry

January

Vol. Price

February

Vol. Price

March

Vol. Price

Quarterly

Total

Avg.

Vol. Price

Sumas, Washington

$14 \quad 3.45$

- N/A

20

1.21

33

2.12

Eastport, Idaho

$0 \quad$ N/A

$0 \quad$ N/A

$3 \quad 1.09$

$3 \quad 1.09$

IGI RESOURCES INC.

CanWest Gas Supply Inc.

WP Natural Gas

Eastport, Idaho

- N/A

- N/A

21.09

2

1.09

IGI RESOURCES INC.

CanWest Gas Supply Inc.

Weyerhauser

Eastport, Idaho

o N/A

- N/A

$3 \quad 1.09$

$3 \quad 1.09$

IGI RESOURCES INC.

CanWest Gas Supply Inc.

Willamette

Eastport, Idaho

- N/A

- N/A

$3 \quad 1.09$

$3 \quad 1.09$

IGI RESOURCES INC.

Cibola Canada Energy Marketing Co.

Aluminum Company of America

Eastport, tdaho

O N/A

$3 \quad 2.20$

- N/A

3

IGI RESOURCES INC.

Cibola Canada Energy Marketing Co.

Boise Cascade Corporation

Eastport, Idaho

$0 \quad$ N/A

$1 \quad 2.20$

- N/A

1

2.20

IGI RESOURCES INC.

Cibola Canada Energy Marketing Co. Cascade Natural Gas

Eastport, Idaho

$1 \quad 1.67$

$8 \quad 2.20$

o N/A

9

2.13

IGI RESOURCES INC.

Cibola Canada Energy Marketing Co.

J.R. Simplot Company

Eastport, Idaho

$5 \quad 1.67$

22

2.20

- N/A

27

2.11

IGI RESOURCES INC.

Cibola Canada Energy Marketing Co. Mountain Fuel

Eastport, Idaho
- N/A

$5 \quad 2.20$

o N/A

$5 \quad 2.20$ 
SHORT-TERM SALES

YEAR: 1997 Quarter: One

Estimated Volumes (MMCF) \& Prices (\$ / MMBTU)
Importer

Seller

Purchaser / End User

IGI RESOURCES INC.

Cibola Canada Energy Marketing Co. PITCO

IGI RESOURCES INC

Cibola Canada Energy Marketing Co.

WP Natural Gas

Point

Entr

January

Vol. Price

February

Vol. Price

March
Vol. Price

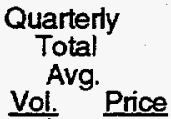

Eastport, Idaho

$0 \quad$ N/A

14

2.20

$1 \quad 1.67$

$0 \quad$ N/A

o N/A

$1 \quad 1.67$

IGI RESOURCES INC

Cibola Canada Energy Marketing Co.

Weyerhauser

Eastport, Idaho

- N/A

$4 \quad 2.20$

o N/A

$4 \quad 2.20$

IGI RESOURCES INC

Cibola Canada Energy Marketing Co. Willamette

Eastport, Idaho

$1 \quad 1.67$

$4 \quad 2.20$

- N/A

$5 \quad 2.07$

IGI RESOURCES INC

Cook Inlet Enercy Supply

Cascade Natural Gas

Eastport, Idaho

o N/A

$1 \quad 1.05$

o N/A

11.05

IGI RESOURCES INC

Cook Inlet Energy Supply

J.R. Simplot Company

IG! RESOURCES INC.

Cook Inlet Energy Supply

Mountain Fuel

Eastport, Idaho

- N/A

$1 \quad 1.05$

- N/A

$1 \quad 1.05$

IGI RESOURCES INC.

Cook Inlet Energy Supply

PITCO

Eastport, Idaho

$0 \quad N / A$

$2 \quad 1.05$

$0 \quad$ N/A

$2 \quad 1.05$

IGI RESOURCES INC.

Cook Inlet Eneroy Supply

Weyerhauser

Eastport, Idaho

o N/A

$1 \quad 1.05$

$0 \quad$ N/A

$1 \quad 1.05$

IGI RESOURCES INC.

Cook Inlet Energy Supply

Willamette

Eastport, Idaho

$0 \quad$ N/A

$1 \quad 1.05$

- N/A

$1 \quad 1.05$

IGI RESOURCES INC.

Crestar Energy

Aluminum Company of America

Eastport, Idaho
$2 \quad 1.73$

$0 \quad$ N/A

- N/A

2

1.73 
SHORT-TERM SALES

YEAR: 1997 Quarter: One

Estimated Volumes (MMCF) \& Prices (\$ / MMBTU)
Importer

Seller

Purchaser / End User

IGI RESOURCES INC.

Crestar Energy

Cascade Natural Gas

IGI RESOURCES INC.

Crestar Energy

J.R. Simplot Company

IGI RESOURCES INC.

Crestar Energy

PITCO

Crestar Energy

Willamette

IGI RESOURCES INC.

El Paso Gas Marketing Canada Inc.

Aluminum Company of America

IGI RESOURCES INC.

El Paso Gas Marketing Canada Inc.

Cascade Natural Gas

IGI RESOURCES INC.

El Paso Gas Marketing Canada Inc. J.R. Simplot Company

IGI RESOURCES INC.

El Paso Gas Marketing Canada Inc. PITCO

Eastport, Idaho

EI Paso Gas Marketing Canada Inc. WP Natural Gas

IGI RESOURCES INC.

El Paso Gas Marketing Canada Inc.

Willamette
Eastport, Idaho

Eastport, Idaho

Eastport, Idaho

Eastport, Idaho

Eastport, Idaho

Eastport, Idaho

Eastport, Idaho

$15 \quad 1.80$

$0 \quad$ N/A

0

N/A

$15 \quad 1.80$

January
Vol. Price

February

Vol. Price

March

Vol. Price

Quarterly

Total

Vol. Price

29

- N/A

- N/A

29

1.73

$0 \quad$ N/A

- N/A

$2 \quad 1.73$

$4 \quad 1.73$

$0 \quad N / A$

- N/A

$4 \quad 1.73$

$4 \quad 1.80$

o N/A

- N/A

$4 \quad 1.80$

$1 \quad 1.80$

- N/A

- N/A

$1 \quad 1.80$
$2 \quad 1.73$
$4 \quad 1.80$

N/A

N/A

4 
SHORT-TERM SALES

YEAR: 1997 Quarter: One

Estimated Volumes (MMCF) \& Prices (\$/MMBTU)
Importer

Seller

Purchaser/End User

IGI RESOURCES INC.

Longview

AIG Trading Corporation

IGI RESOURCES INC.

Longview

Amalgamated Sugar Company

IGI RESOURCES INC

Longview

Amoco Trading

IGI RESOURCES INC

Longview

Armstrong World Industries, Inc.

IGI RESOURCES INC.

Longview

Ash Grove Cement

IGI RESOURCES INC.

Longview

Basic American Foods

IGI RESOURCES INC.

Longview

Birmingham Steel

Sumas, Washington

Sumas, Washington

IGI RESOURCES INC.

Longview

Boeing Company

IGI RESOURCES INC.

Longview

Boise Cascade Corporation

IGI RESOURCES INC.

Longview

Camation Daines

Sumas, Washington

- N/A

o N/A

$3 \quad 1.13$

1.13

58

IGI RESOURCES INC.

Longview

Cascade Natural Gas

$\begin{gathered}\text { January } \\ \text { Vebruary } \\ \text { Vol. Price Price }\end{gathered}$
Vol.

March
Vol. Price

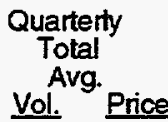

Sumas, Washington

$0 \quad$ N/A

O N/A

1

1.13

1.13

Sumas, Washington

o. N/A

o N/A

$9 \quad 1.13$

$9 \quad 1.13$

Sumas, Washington

O N/A

23.05

$7 \quad 1.13$

10

1.60

Sumas, Washington

o. N/A

$40 \quad 2.85$

18

$6 \quad 0.59$

Sumas, Washington

$\begin{array}{llll}0 & \text { N/A } & 18 & 3.05\end{array}$

$0 \quad N / A$

18

3.05 
SHORT-TERM SALES

YEAR: 1997 Quarter: One

Estimated Volumes (MMCF) \& Prices (\$ / MMBTU)
Importer

Seller

Purchaser/ End User

IGI RESOURCES INC.

Longview

Chevron U.S.A. Inc.

IGI RESOURCES INC.

Longview

Columbia Beverage

IGI RESOURCES INC.

Longview

Daring Delaware

IGI RESOURCES INC.

Longview

Davis Wire

IGI RESOURCES INC.

Longview

Dyno Polymers

IGI RESOURCES INC.

Longview

Evanite Fiber

IGI RESOURCES INC.

Longview

Fircrest Schools

IGI RESOURCES INC.

Longview

General Chemical

IGI RESOURCES INC.

Longview

Inland Natural Gas

IGI RESOURCES INC.

Longview

J.H. Gypsum

IGI RESOURCES INC.

Longview

J.R. Simplot Company
Point

of

Entry

Sumas, Washington

Sumas, Washington

- N/A

- N/A

$1 \quad 1.13$

1

1.13

Sumas, Washington

o N/A

O N/A

$1 \quad 1.13$

1

1.13

Sumas, Washington

- N/A

- N/A

11.13

1

1.13

Sumas, Washington

- N/A

- N/A

$1 \quad 1.13$

1

1.13

Sumas, Washington

- N/A

- N/A

2. 1.13

2

1.13

Sumas, Washington

- N/A

- N/A

21.13

$2 \quad 1.13$

Sumas, Washington

- N/A

o N/A

1

1.13

1

1.13

Sumas, Washington

O N/A

o N/A

15

1.13

15

1.13

Sumas, Washington

- N/A

$0 \quad N / A$

$5 \quad 1.13$

5

1.13

Sumas, Washington
$30 \quad 3.05$

17

1.13

47

2.37 
SHORT-TERM SALES

YEAR: 1997 Quarter: One

Estimated Volumes (MMCF) \& Prices (\$ / MMBTU)

Importer

Seller

Purchaser / End User

IGI RESOURCES INC.

Longview

Jorgenson Forge

IGI RESOURCES INC.

Longview

Kings Meat

IGI RESOURCES INC.

Longview

Lakeside

IGI RESOURCES INC.

Longview

Lamb Weston

Sumas, Washington

IGI RESOURCES INC.

Longview

M.A. Segale

IGI RESOURCES INC.

Longview

MTVHS

[G] RESOURCES INC.

Longview

Mountain Fuel

IGI RESOURCES INC.

Longview

Northwest Cooperage

IGI RESOURCES INC.

Longview

Ore-lda

IGI RESOURCES INC.

Longview

Oregon Potato

Sumas, Washington

IGI RESOURCES INC

Longview

Point
of

Entry

Sumas, Washington

Sumas, Washington

Sumas, Washington

Sumas, Washington

Sumas, Washington

Sumas, Washington

Sumas, Washington

Sumas, Washington
- N/A

13.05

- N/A

13.05

January

Vol. Price

February

Vol. Price

March

Vol. Price

Quarterly
Total

Avg.

Vol. Price

- N/A

- N/A

11.13

$1 \quad 1.13$

O N/A

$1 \quad 1.13$

1

$0 \quad$ N/A

- N/A

10

1.13

10

1.13

- N/A

- N/A

$3 \quad 1.13$

3

1.13

- N/A

- N/A

$1 \quad 1.13$

$1 \quad 1.13$

$0 \quad$ N/A

o N/A

$1 \quad 1.13$

1

1.13

$0 \quad$ N/A

- N/A

$2 \quad 1.13$

$2 \quad 1.13$

o N/A

$2 \quad 1.13$

21.13
Overall Laundry

Sumas, Washington

- N/A

- N/A

1 
SHORT-TERM SALES

YEAR: 1997 Quarter: One

Estimated Volumes (MMCF) \& Prices (\$ / MMBTU)

Importer

Seller

Purchaser / End User

IGI RESOURCES INC.

Longview

PABST

IGI RESOURCES INC

Longview

Pendelton

IGI RESOURCES INC.

Longview

Pierce Transit

IGI RESOURCES INC.

Longview

Portland General Electric Company

Sumas, Washington

IGI RESOURCES INC.

Longview

Rainy River

Sumas, Washington

IGI RESOURCES INC.

Longview

Roseburg Lumber

IGI RESOURCES INC.

Longview

Seattle Snohomish

IGI RESOURCES INC.

Longview

Smurfit Newsprint Corporation

IGI RESOURCES INC.

Longview

Southwest Gas Corporation

IGI RESOURCES INC.

Longview

Teledyne

IGI RESOURCES INC.

Longview

Todds Shipyards

Point

Entry

Sumas, Washington

Sumas, Washington

Sumas, Washington

Sumas, Washington

Sumas, Washington

Sumas, Washington

Sumas, Washington

Sumas, Washington
Sumas, Washington
- N/A

- N/A

11.13

$1 \quad 1.13$

o N/A

$4 \quad 3.05$

$0 \quad$ N/A

$4 \quad 3.05$

- N/A

- N/A

$3 \quad 1.13$

$3 \quad 1.13$

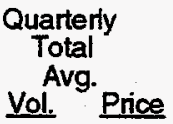

1.13

$1 \quad 1.13$

$8 \quad 3.05$

$2 \quad 1.13$

o N/A

o N/A

1. 1.13

$1 \quad 1.13$

o N/A

$0 \quad$ N/A

$3 \quad 1.13$

3

1.13

$0 \quad$ N/A

o N/A

21.13

2

1.13

$0 \quad$ N/A

1

1.13

1

1.13 


\section{SHORT-TERM SALES \\ YEAR: 1997 Quarter: One \\ Estimated Volumes (MMCF) \& Prices (\$/MMBTU)}

Importer

Seller

Purchaser / End User

IGI RESOURCES INC.

Longview

US Oil \& Refining

IGI RESOURCES INC.

Longview

Valley Medical

Sumas, Washington

IGI RESOURCES INC.

Longview

WP Natural Gas

Sumas, Washington

Sumas, Washington

LOI RESO

Washington Natural Gas Company

IGI RESOURCES INC.

Longview

Washington Water Power Company

IGI RESOURCES INC.

Longview

Weyerhauser

Sumas, Washington

$0 \quad$ N/A

$3 \quad$ N/A

$5 \quad 1.13$

8

0.73

IGI RESOURCES INC.

Longview

Willamette

Sumas, Washington

- N/A

$24 \quad 3.05$

o N/A

24

3.05

IGI RESOURCES INC.

Longview

Woodworth

Sumas, Washington

o. N/A

- N/A

$2 \quad 1.13$

$2 \quad 1.13$

IGI RESOURCES INC

Panhandle Energy Marketing

Aluminum Company of America

IGI RESOURCES INC.

Panhandle Energy Marketing

Barret Fuels

Eastport, Idaho

- N/A

0

N/A

$33 \quad 1.15$

33

1.15

IGI RESOURCES INC.

Panhandle Energy Marketing

Basic American foods
Eastport, Idaho
- N/A

$3 \quad 1.15$
$3 \quad 1.15$ 
SHORT-TERM SALES

YEAR: 1997 Quarter: One

Estimated Volumes (MMCF) \& Prices (\$ / MMBTU)
Importer

Seller

Purchaser / End User

IG! RESOURCES INC.

Panhandle Energy Marketing Boise Cascade Corporation

IGI RESOURCES INC.

Panhandle Energy Marketing Borden

IGI RESOURCES INC.

Panhandle Energy Marketing

Cascade Natural Gas

IGI RESOURCES INC.

Panhandle Energy Marketing Chevron U.S.A. Inc.

IGI RESOURCES INC.

Panhandle Energy Marketing ConAgra Energy

IGI RESOURCES INC

Panhandle Energy Marketing ESCO Corporation

IGI RESOURCES INC.

Panhandle Energy Marketing Eagle Picher

IGI RESOURCES INC.

Panhandle Energy Marketing

Evanite Fiber

Eastport, Idaho

IGI RESOURCES INC

Panhandle Energy Marketing J.R. Simplot Company

IGI RESOURCES INC.

Panhandle Energy Marketing Lakeside

Eastport, Idaho

$0 \quad N / A$

$2 \quad 1.59$

- N/A

$2 \quad 1.59$

IGI RESOURCES INC.

Panhandle Energy Marketing Lamb Weston

Point

Entry

January

o N/A

$5 \quad 1.59$

$5 \quad 1.16$

10

1.38

Eastport, Idaho

o. N/A

$0 \quad$ N/A

21.15

$2 \quad 1.15$

Eastport, Idaho

$56 \quad 1.47$

$67 \quad 1.59$

73

1.15

197

1.39

Eastport, Idaho

$1 \quad 1.47$

- N/A

$1 \quad 1.15$

$3 \quad 1.31$

Eastport, Idaho

$1 \quad 1.24$

$1 \quad 1.24$

- N/A

$1 \quad 1.24$

Eastport, Idaho

Eastport, Idaho

232

1.59

Eastport, Idaho $\begin{array}{llll}0 & \text { N/A } & 1 & 1.24\end{array}$

- N/A

1

1.24 
SHORT-TERM SALES

YEAR: 1997 Quarter: One

Estimated Volumes (MMCF) \& Prices (\$ / MMBTU)
Importer

Seller

Purchaser/ End User

IGI RESOURCES INC.

Panhandle Energy Marketing

Legacy Health System

IGI RESOURCES INC.

Panhandle Energy Marketing

Mountain Fuel

Eastport, Idaho

January

Entry

Eastport, Idaho

$0 \quad$ N/A

February

Vol. Price
March

Vol. Price
Quarterly

Total

Vol. Price

IGI RESOURCES INC

Panhandle Energy Marketing NORPAC

Eastport, Idaho

$0 \quad$ N/A

o N/A

$2 \quad 1.15$

$2 \quad 1.15$

IGI RESOURCES INC

Panhandle Energy Marketing PITCO

Eastport, Idaho

$18 \quad 1.47$

$113 \quad 1.59$

- N/A

131

1.57

IGI RESOURCES INC.

Panhandle Energy Marketing

Pacific Gas \& Electric (UEG)

Eastport, Idaho

$0 \quad$ N/A

O N/A

$48 \quad 1.15$

48

1.15

IGI RESOURCES INC.

Panhandle Energy Marketing

PanEnergy Trading \& Marketing Svcs.

Eastport, Idaho

$0 \quad$ N/A

$0 \quad N / A$

114

1.15

114

1.15

IGI RESOURCES INC

Panhandle Energy Marketing

Pendelton

Eastport, Idaho

$0 \quad N / A$

- N/A

$1 \quad 1.15$

$1 \quad 1.15$

IGI RESOURCES INC

Panhandle Energy Marketing WP Natural Gas

Eastport, Idaho

$29 \quad 1.47$

0. N/A

28

1.15

57. 1.31

IGI RESOURCES INC

Panhandle Energy Marketing

Washington Natural Gas Company

Eastport, Idaho

$0 \quad$ N/A

$2 \quad 1.59$

O N/A

$2 \quad 1.59$

IGI RESOURCES INC

Panhandle Energy Marketing

Washington Water Power Company

Eastport, Idaho

$2 \quad 1.47$

1. 1.59

$4 \quad 1.15$

7

1.32

IGI RESOURCES INC

Panhandle Energy Marketing

Weyerhauser

Eastport, Idaho

$33 \quad 1.59$

46

1.15

83

1.34 


\section{SHORT-TERM SALES \\ YEAR: 1997 Quarter: One \\ Estimated Volumes (MMCF) \& Prices (\$ / MMBTU)}

Importer

Seiler

Purchaser / End User

IGI RESOURCES INC.

Panhandle Energy Marketing

Willamette

Eastport, Idaho

Sumas, Washington

RESOURCES INC.

Shell Canada Limited

AIG Trading Corporation

IGI RESOURCES INC.

Shell Canada Limited

Aluminum Company of America

IGI RESOURCES INC

Shell Canada Limited

Amalgamated Sugar Company

IGI RESOURCES INC

Shell Canada Limited

Amoco Trading

IGI RESOURCES INC

Shell Canada Limited

Amstrong Worid Industries, Inc.

IGI RESOURCES INC

Shell Canada Limited

Ash Grove Cement

IGI RESOURCES INC.

Shell Canada Limited

Basic American Foods

IGI RESOURCES INC.

Shell Canada Limited

Birmingham Steel

IGI RESOURCES INC.

Shell Canada Limited

Boeing Company

Sumas, Washington

$0 \quad$ N/A

o N/A

17

1.07

17

1.07

IGI RESOURCES INC.

Shell Canada Limited

Boise Cascade Corporation

Point

Entry

January

Vol. Price

February

Vol. Price

Vol.

$37 \quad 1.15$

131

1.41

Eastport, Idaho

$4 \quad 2.08$

$0 \quad$ N/A

- N/A

4

2.08

Sumas, Washington

Sumas, Washington

$3 \quad 4.17$

4

$3 \quad 1.07$

20

1.77

Sumas, Washington

Sumas, Washington

Sumas, Washington

$\begin{array}{ll}9 & 4.17\end{array}$

$5 \quad 2.39$

$5 \quad 1.07$

19

2.85

Sumas, Washington
$8 \quad 4.17$

69

2.39

1.07

110

2.13 
SHORT-TERM SALES

YEAR: 1997 Quarter: One

Estimated Volumes (MMCF) \& Prices (\$ / MMBTU)

Importer

Seller

Purchaser / End User

IGI RESOURCES INC. Shell Canada Limited

Camation Dairies

IGI RESOURCES INC. Shell Canada Limited

Cascade Natural Gas

IGI RESOURCES INC. Shell Canada Limited Cascade Natural Gas

IGI RESOURCES INC Shell Canada Limited Cenral Premix

Sumas, Washington

IGI RESOURCES INC Shell Canada Limited Chevron U.S.A. Inc.

IGI RESOURCES INC Shell Canada Limited City of Buckley, OR

IGI RESOURCES INC. Shell Canada Limited Columbia Beverage

IGI RESOURCES INC

Shell Canada Limited

Darting Delaware

IGI RESOURCES INC Shell Canada Limited Davis Wire

Sumas, Washington

IGI RESOURCES INC. Shell Canada Limited Draper Farms

IGI RESOURCES INC Shell Canada Limited Dyno Polymers

Point

Entry

Sumas, Washington

Eastport, Idaho

Sumas, Washington

Sumas, Washington

Sumas, Washington

Sumas, Washington

Sumas, Washington

Sumas, Washington
January

vol. Price

February

Vol. Price

March

Vol. Price

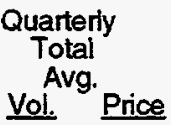

$\begin{array}{ll}9 & 4.17\end{array}$

- N/A

$5 \quad 1.07$

13

3.10

$12 \quad 2.08$

- N/A

- N/A

12

2.08

$\begin{array}{ll}38 & 4.17\end{array}$

31

2.39

- N/A

68

3.37

- N/A

o. N/A

$\begin{array}{ll}7 & 1.07\end{array}$

$7 \quad 1.07$

$0 \quad$ N/A

- N/A

$1 \quad 1.07$

1

- N/A

N/A

$2 \quad 1.07$

$2 \quad 1.07$

- N/A

- N/A

$2 \quad 1.07$

$2 \quad 1.07$

14.17

- N/A

$0 \quad$ N/A

1

4.17
- N/A

- N/A

1

1.07 
SHORT-TERM SALES

YEAR: 1997 Quarter: One

Estimated Volumes (MMCF) \& Prices (\$ / MMBTU)

Importer

Seller

Purchaser / End User

IGI RESOURCES INC.

Shell Canada Limited

Elanbaas

Sumas, Washington

Point

of

Entry

Sumas, Washington

GI RESOURCES INC.

Shell Canada Limited

Evanite Fiber

IGI RESOURCES INC. Shell Canada Limited

Fircrest Schools

[GI RESOURCES INC.

Shell Canada Limited

General Chemical

IGI RESOURCES INC. Shell Canada Limited

Heath

IGI RESOURCES INC Shell Canada Limited

Inland Natural Gas

IGI RESOURCES INC. Shell Canada Limited Interox

Sumas, Washington

13

4.17

- N/A

o N/A

13

4.17

IGI RESOURCES INC. Shell Canada Limited

J.H. Gypsum

Sumas, Washington

o. N/A

0

N/A

10

1.07

10

1.07

IGI RESOURCES INC. Shell Canada Limited J.R. Simplot Company

IGI RESOURCES INC. Shell Canada Limited J.R. Simplot Company

IGI RESOURCES INC. Shell Canada Limited Jorgenson Forge
Sumas, Washington
Quarterly
Total

Avg

Vol. Price

Vol. Price

$3 \quad 1.07$

$4 \quad 1.07$

$1 \quad 1.07$

Eastport, Idaho

$49 \quad 2.08$

- N/A

o N/A

$49 \quad 2.08$

Sumas, Washington

$\begin{array}{ll}69 & 4.17\end{array}$

52

2.39

$30 \quad 1.07$

151

2.94

- N/A

$5 \quad 1.07$

$5 \quad 1.07$ 
SHORT-TERM SALES

YEAR: 1997 Quarter: One

Estimated Volumes (MMCF) \& Prices (\$ / MMBTU)
Importer

Seller

Purchaser / End User

IGI RESOURCES INC.

Shell Canada Limited

Kings Meat

IGI RESOURCES INC.

Shell Canada Limited

Lakeside

Sumas, Washington

[GI RESOURCES INC.

Shell Canada Limited

Lamb Weston

IGI RESOURCES INC.

Shell Canada Limited

M.A. Segale

Sumas, Washington

IGI RESOURCES INC.

Shell Canada Limited

MTVHS

Sumas, Washington

IGI RESOURCES INC.

Shell Canada Limited

Mountain Fuel

Sumas, Washington

Sumas, Washington

IGI RESOURCES INC.

Shell Canada Limited

Noram Energy Services

IGI RESOURCES INC.

Shell Canada Limited

Northstar Energy

IGI RESOURCES INC. Shell Canada Limited Northwest Cooperage

IGI RESOURCES INC Shell Canada Limited Ore-lda
Sumas, Washington

Sumas, Washington

Sumas, Washington

Sumas, Washington
14.17

$0 \quad$ N/A

N/A

1

4.17

$1 \quad 4.17$

$0 \quad$ N/A

$1 \quad 1.07$

1
- N/A

$2 \quad 2.39$
Quarterty

Total Vol.

$1 \quad 1.07$

$2 \quad 1.07$

$31 \quad 2.35$

$1 \quad 1.07$

$2 \quad 1.07$

o. N/A

0

N/A

$3 \quad 1.07$

$3 \quad 1.07$
$0 \quad$ N/A

2 
SHORT-TERM SALES

YEAR: 1997 Quarter: One

Estimated Volumes (MMCF) \& Prices (\$ / MMBTU)

Importer

Seller

Purchaser / End User

IGI RESOURCES INC.

Shell Canada Limited

Oregon Potato

IGI RESOURCES INC.

Shell Canada Limited

Overall Laundry

IGI RESOURCES INC.

Shell Canada Limited

PABST

IGI RESOURCES INC.

Shell Canada Limited

PITCO

Eastport, Idaho

$4 \quad 2.08$

O N/A

o N/A

4

2.08

IGI RESOURCES INC.

Shell Canada Limited

Pendelton

Sumas, Washington

14.17

$0 \quad$ N/A

$2 \quad 1.07$

$3 \quad 2.30$

IGI RESOURCES INC.

Shell Canada Limited

Pierce Transit

Sumas, Washington

$0 \quad$ N/A

- N/A

21.07

$2 \quad 1.07$

IGI RESOURCES INC.

Shell Canada Limited

Portland General Electric Company

Sumas, Washington

$31 \quad 4.17$

13

2.39

0

N/A

44

3.63

IGI RESOURCES INC.

Shell Canada Limited

Rainy River

Sumas, Washington

$0 \quad$ N/A

o N/A

$3 \quad 1.07$

$3 \quad 1.07$

IGI RESOURCES INC.

Shell Canada Limited

Redhook

Sumas, Washington

$0 \quad$ N/A

- N/A

$1 \quad 1.07$

$1 \quad 1.07$

IGI RESOURCES INC.

Shell Canada Limited

Roseburg Lumber

Sumas, Washington

$0 \quad$ N/A

$\begin{array}{ll}7 & 2.39\end{array}$

$1 \quad 1.07$

7

2.25

IGI RESOURCES INC.

Shell Canada Limited

Seattle Snohomish
Sumas, Washington

o N/A

$0 \quad N / A$

21.07

2

1.07 
SHORT-TERM SALES

YEAR: 1997 Quarter: One

Estimated Volumes (MMCF) \& Prices (\$ / MMBTU)
Importer

Seller

Purchaser / End User

IGI RESOURCES INC.

Shell Canada Limited

Smurfit Newsprint Corporation

IGI RESOURCES INC.

Shell Canada Limited

Southwest Gas Corporation

IGI RESOURCES INC.

Shell Canada Limited

Teledyne

IGI RESOURCES INC.

Shell Canada Limited

Texaco Gas Marketing Inc.

IGI RESOURCES INC.

Shell Canada Limited

Todds Shipyards

IGI RESOURCES INC.

Shell Canada Limited

TransOcean Products, Inc.

Sumas, Washington

IGI RESOURCES INC.

Shell Canada Limited

US Oil \& Refining

IGI RESOURCES INC.

Shell Canada Limited

Valley Medical

IGI RESOURCES INC.

Shell Canada Limited

WP Natural Gas

Eastport, Idaho

$6 \quad 2.08$

$0 \quad$ N/A

o N/A

$6 \quad 2.08$

IGI RESOURCES INC.

Shell Canada Limited

WP Natural Gas

IGI RESOURCES INC.

Shell Canada Limited

Washington Energy Gas Marketing Co.

Point

Entry

January

February

Vol. Price

March

Vol. Price

Quarterty

Total

Avg. Price

Sumas, Washington

$0 \quad N / A$

- N/A

$5 \quad 1.07$

$5 \quad 1.07$

Sumas, Washington

$13 \quad 4.17$

O N/A

$4 \quad 1.07$

16

3.50

Sumas, Washington

- N/A

$0 \quad$ N/A

$5 \quad 1.07$

$5 \quad 1.07$

Sumas, Washington

14.17

- N/A

o N/A

14.17

Sumas, Washington

- N/A

- N/A

$2 \quad 1.07$

21.07

Sumas, Washington

- N/A

- N/A

$5 \quad 1.07$

$5 \quad 1.07$

Sumas, Washington

- N/A

$0 \quad$ N/A

$1 \quad 1.07$

$1 \quad 1.07$

Sumas, Washington

$7 \quad 4.17$

0

N/A

1.07

21

Sumas, Washington
$4 \quad 4.17$

- N/A

- NA

4

4.17 
SHORT-TERM SALES

YEAR: 1997 Quarter: One

Estimated Volumes (MMCF) \& Prices (\$ / MMBTU)
Importer

Seller

Purchaser / End User

IGI RESOURCES INC.

Shell Canada Limited

Washington Natural Gas Company

IGI RESOURCES INC.

Shell Canada Limited

Washington Water Power Company

IGI RESOURCES INC.

Shell Canada Limited

Weyerhauser

IGI RESOURCES INC.

Shell Canada Limited

Weyerhauser

Sumas, Washington

$\begin{array}{ll}6 & 4.17\end{array}$

$5 \quad 2.39$

$9 \quad 1.07$

20

2.28

IGI RESOURCES INC.

Shell Canada Limited

Willamette

Eastport, Idaho

$13 \quad 2.08$

- N/A

$0 \quad$ N/A

13

2.08

IGI RESOURCES INC.

Shell Canada Limited

Willamette

Sumas, Washington

$44 \quad 4.17$

42

2.39

$0 \quad$ N/A

85

3.30

IGI RESOURCES INC.

Shell Canada Limited

Woodworth

Sumas, Washington

$0 \quad$ N/A

$0 \quad$ N/A

$3 \quad 1.07$

3

1.07

IGI RESOURCES INC.

Suncor Inc.

Aluminum Company of America

Eastport, Idaho

$2 \quad 1.16$

$3 \quad 1.16$

$2 \quad 1.16$

7

1.16

IGI RESOURCES INC.

Suncor lnc.

Barret Fuels

Eastport, Idaho

$0 \quad$ N/A

- N/A

$4 \quad 1.16$

4

1.16

IGI RESOURCES INC.

Suncor Inc.

Boise Cascade Corporation

Eastport, Idaho

O N/A

$1 \quad 1.16$

$1 \quad 1.16$

1

1.16

IGI RESOURCES INC.

Suncor Inc.

Cascade Natural Gas
Eastport, Idaho
$7 \quad 1.16$

$8 \quad 1.16$

1.16

24

1.16 


\section{SHORT-TERM SALES \\ YEAR: 1997 Quarter: One \\ Estimated Volumes (MMCF) \& Prices (\$ / MMBTU)}

Importer

Seller

Purchaser / End User

IGI RESOURCES INC.

Suncor Inc.

Evanite Fiber

IGI RESOURCES INC

Suncor Inc.

J.R. Simplot Company

IGI RESOURCES INC.

Suncor lnc.

Mountain Fuel

Eastport, Idaho

IGI RESOURCES INC

Suncor Inc.

PITCO

IGI RESOURCES INC

Suncor Inc.

Pacific Gas \& Electric (UEG)

Eastport, Idaho

Eastport, Idaho

Eastport, Idaho

Eastport, Idaho

Eastport, Idaho

Eastport, Idaho

Eastport, Idaho

IGI RESOURCES INC

Suncor lnc.

PanEnergy Trading \& Marketing Svcs.

IGI RESOURCES INC.

Suncor lnc.

WP Natural Gas

IGI RESOURCES INC.

Suncor Inc.

Washington Water Power Company

IGI RESOURCES INC

Suncor Inc.

IGI RESOURCES INC.

Suncor inc.

Wiltamette

IGI RESOURCES INC.

Talisman Energy, Inc.

Cascade Natural Gas

Eastport, Idaho
January

Vol. Price

February

Vol. Price

March

Vol. Price

Quarterly

Total

Avg.

Vol. Price

Weyerhauser
$30 \quad 1.16$

$21 \quad 1.16$

75

1.16

- N/A

$5 \quad 1.16$

$0 \quad N / A$

$5 \quad 1.16$

21.16

$14 \quad 1.16$

- N/A

16

1.16

- N/A

o N/A

$5 \quad 1.16$

$5 \quad 1.16$

$0 \quad$ N/A

- N/A

$13 \quad 1.16$

13

1.16

$4 \quad 1.16$

- N/A

$3 \quad 1.16$

$7 \quad 1.16$

- N/A

$4 \quad 1.16$

$5 \quad 1.16$

$9 \quad 1.16$

$8 \quad 1.16$

$4 \quad 1.16$

$4 \quad 1.16$

16

1.16

- N/A

o N/A

$1 \quad 1.27$
$23 \quad 1.16$ 


\section{SHORT-TERM SALES \\ YEAR: 1997 Quarter: One \\ Estimated Volumes (MMCF) \& Prices ( $/$ MMBTU)}

Importer

Seller

Purchaser / End User

IGI RESOURCES INC.

Talisman Energy, Inc.

J.R. Simplot Company

IGI RESOURCES INC.

Talisman Energy, Inc.

J.R. Simplot Company

IGI RESOURCES INC.

Talisman Energy, Inc.

PanEnergy Trading \& Marketing Svcs.

IGI RESOURCES INC.

TransCanada Gas Services Limited

J.R. Simplot Company

IGI RESOURCES INC.

Wascana Energy Marketing Inc.

Aluminum Company of America

IGI RESOURCES INC.

Wascana Energy Marketing Inc.

American Fine Foods

Eastport, Idaho

IGI RESOURCES INC.

Wascana Energy Marketing Inc.

Barret Fuels

Eastport, Idaho

Eastport, Idaho

IGI RESOURCES INC.

Wascana Energy Marketing Inc.

Basic American Foods

IGI RESOURCES INC

Wascana Energy Marketing Inc.

Boise Cascade Corporation

IGI RESOURCES INC.

Wascana Energy Marketing Inc.

Borden

Eastport, idaho

- N/A

- N/A

$1 \quad 1.28$

$1 \quad 1.28$

IGI RESOURCES INC.

Wascana Energy Marketing Inc.

Bullseye Glass

Point

Entry

January

Vol. Price

February

Vol. Price

March
Vol. Price

Quarterly
Total

Avg.

Vol. Price

Eastport, Idaho

- N/A

- N/A

$1 \quad 1.27$

$1 \quad 1.27$

Sumas, Washington

234

4.36

247

2.50

305

785

2.51

Eastport, Idaho

- N/A

- N/A

$1 \quad 1.27$

$1 \quad 1.27$

Eastport, Idaho

- N/A

$1 \quad 1.09$

- N/A

1

1.09

Eastport, Idaho

$11 \quad 1.70$

$13 \quad 2.07$

$12 \quad 1.28$

36

1.69

$0 \quad 1.70$

$0 \quad 2.07$

- N/A

1

1.87

Eastport, Idaho

o N/A

$3 \quad 2.07$

$3 \quad 1.28$

$6 \quad 1.68$

Eastport, Idaho
$0 \quad 1.70$

0.07
0

$\begin{array}{ll}0 & 1.28\end{array}$

1

1.82 
SHORT-TERM SALES

YEAR: 1997 Quarter: One

Estimated Volumes (MMCF) \& Prices (\$ / MMBTU)
Importer

Seller

Purchaser / End User

IGI RESOURCES INC.

Wascana Energy Marketing inc.

Cascade Natural Gas

IGI RESOURCES INC.

Wascana Energy Marketing Inc.

Chevron U.S.A. Inc.

IGI RESOURCES INC.

Wascana Energy Marketing inc. ConAgra Energy

Eastport, Idaho

IGI RESOURCES INC.

Wascana Energy Marketing Inc. ESCO Corporation

IGI RESOURCES INC.

Wascana Energy Marketing Inc. Eagle Picher

Eastport, Idaho

IGI RESOURCES INC.

Wascana Energy Marketing Inc.

Evanite Fiber

Eastport, Idaho

IGI RESOURCES INC.

Wascana Energy Marketing Inc.

J.R. Simplot Company

IGI RESOURCES INC.

Wascana Energy Marketing Inc. Lakeside

Eastport, Idaho

IGI RESOURCES INC.

Wascana Energy Marketing Inc.

Lamb Weston

Eastport, Idaho

- N/A

12.07

2.07

- N/A

1

2.07

IGI RESOURCES INC.

Wascana Energy Marketing Inc.

Legacy Health System

Eastport, Idaho

$0 \quad$ N/A

12.07

- N/A

12.07

IGI RESOURCES INC.

Wascana Energy Marketing Inc.

Mountain Fuel

Eastport, Idaho

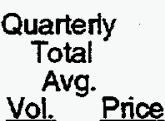

16

1.68

$2 \quad 1.51$

Eastport, Idaho

$147 \quad 1.70$

112

1.28

364

1.69

- N/A

(.)

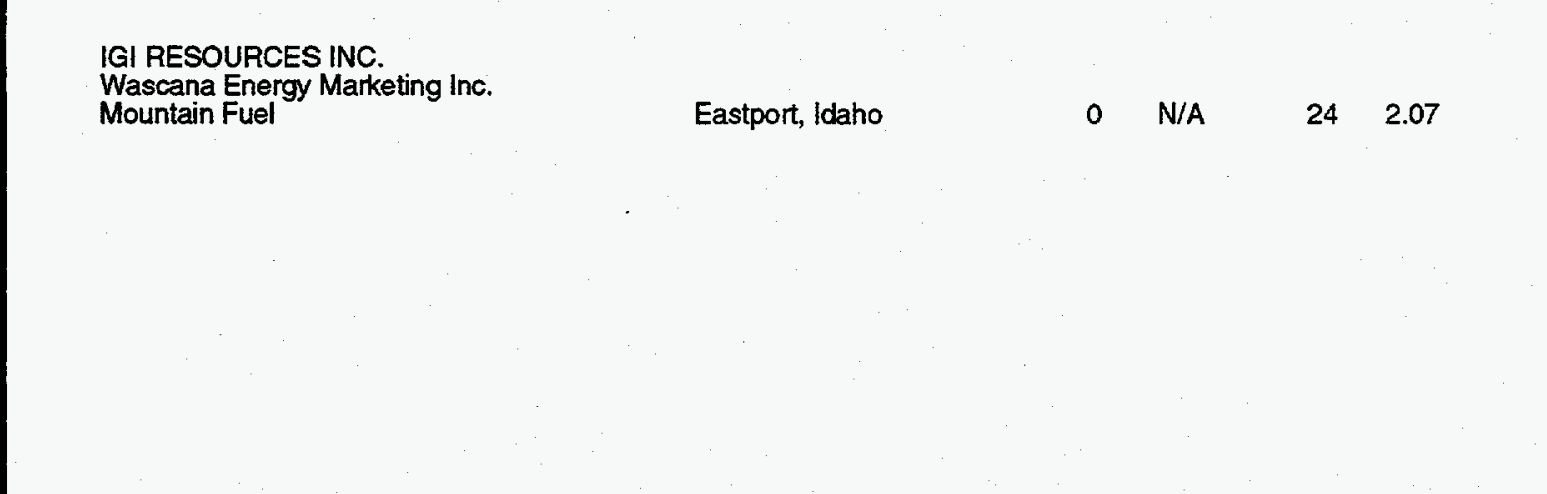


SHORT-TERM SALES

YEAR: 1997 Quarter: One

Estimated Volumes (MMCF) \& Prices (\$/MMBTU)
Importer

Seller

Purchaser / End User

IGI RESOURCES INC.

Wascana Energy Marketing inc. NORPAC

IGI RESOURCES INC.

Wascana Energy Marketing Inc.

PITCO

IGI RESOURCES INC.

Wascana Energy Marketing Inc.

Pacific Gas \& Electric (UEG)

IGI RESOURCES INC.

Wascana Energy Marketing Inc.

PanEnergy Trading \& Marketing Sves. Eastport, Idaho

- N/A

$0 \quad$ N/A

$61 \quad 1.28$

$61 \quad 1.28$

IGI RESOURCES INC.

Wascana Energy Marketing inc.

Pendelton

Eastport, Idaho

- N/A

- N/A

$1 \quad 1.28$

$1 \quad 1.28$

IGI RESOURCES INC.

Wascana Energy Marketing Inc.

WP Natural Gas

Eastport, Idaho

$18 \quad 1.70$

O N/A

$15 \quad 1.28$

33

1.51

IGI RESOURCES INC.

Wascana Energy Marketing Inc.

Washington Natural Gas Company

IGI RESOURCES INC.

Wascana Energy Marketing Inc.

Washington Water Power Company

Eastport, Idaho

$1 \quad 1.70$

$1 \quad 2.07$

$2 \quad 1.28$

4

1.57

IGI RESOURCES INC.

Wascana Energy Marketing Inc.

Weyerhauser

Eastport, Idaho

$2 \quad 1.70$

20

2.07

25

1.28

47

1.63

IGI RESOURCES INC.

Wascana Energy Marketing Inc.

Willamette

Eastport, Idaho

$39 \quad 1.70$

19

2.07

20

1.28

$78 \quad 1.68$

IGI RESOURCES INC.

Westcoast Gas Services Inc.

AIG Trading Corporation
Sumas, Washington
- N/A

o N/A

43

1.07

43

1.07 


\section{SHORT-TERM SALES \\ YEAR: 1997 Quarter: One \\ Estimated Volumes (MMCF) \& Prices (\$ / MMBTU)}

Importer

Seller

Purchaser / End User

IGI RESOURCES INC.

Westcoast Gas Services Inc.

Amalgamated Sugar Company

IGI RESOURCES INC.

Westcoast Gas Services Inc.

Amoco Trading

Point

of

January

Vol. Price

February

Vol. Price

March

Vol. Price

Quarterly

Total

Avg.

Vol. Price

Sumas, Washington

0 N/A

$0 \quad$ N/A

$7 \quad 1.07$

$\begin{array}{ll}7 & 1.07\end{array}$

Sumas, Washington

- N/A

o N/A

$64 \quad 1.07$

$64 \quad 1.07$

IGI RESOURCES INC.

Westcoast Gas Services Inc.

Amnstrong World Industries, Inc.

IGI RESOURCES INC.

Westcoast Gas Senvices Inc.

Ash Grove Cement

Sumas, Washington

18

4.17

$12 \quad 2.39$

$11 \quad 1.07$

$42 \quad 2.82$

IGI RESOURCES INC.

Westcoast Gas Services Inc.

Basic American Foods

Sumas, Washington

- N/A

O N/A

$21 \quad 1.07$

$21 \quad 1.07$

IGI RESOURCES INC.

Westcoast Gas Services Inc.

Birmingham Steel

Sumas, Washington

36

4.17

14

2.39

$22 \quad 1.07$

72

2.88

IGI RESOURCES INC.

Westcoast Gas Services Inc.

Boeing Company

Sumas, Washington

$0 \quad$ N/A

- N/A

$67 \quad 1.07$

$67 \quad 1.07$

IGI RESOURCES INC.

Westcoast Gas Services Inc.

Boise Cascade Comoration

IGI RESOURCES INC.

Westcoast Gas Services inc.

Camation Dairies

Sumas, Washington

35

4.17

$0 \quad$ N/A

$18 \quad 1.07$

54

IGI RESOURCES INC.

Westcoast Gas Services Inc.

Cascade Natural Gas

Sumas, Washington

$151 \quad 4.17$

$90 \quad 2.39$

- N/A

240

IGI RESOURCES INC.

Westcoast Gas Services inc.

Cenral Premix

Sumas, Washington
$0 \quad$ N/A

0

N/A

$3 \quad 1.07$

$3 \quad 1.07$ 


\section{SHORT-TERM SALES \\ YEAR: 1997 Quarter: One \\ Estimated Volumes (MMCF) \& Prices (\$ / MMBTU)}

Importer

Seller

Purchaser / End User

IGI RESOURCES INC.

Westcoast Gas Services Inc.

Chevron U.S.A. Inc.

IGI RESOURCES INC.

Westcoast Gas Services Inc. Cintas

Sumas, Washington

Sumas, Washington

Westcoast Gas Services Inc.

City of Buckley, OR

IGI RESOURCES INC.

Westcoast Gas Services Inc.

Columbia Beverage

IGI RESOURCES INC.

Westcoast Gas Services Inc. Darling Delaware

Sumas, Washington

$0 \quad N / A$

$7 \quad 1.07$

$7 \quad 1.07$

IGI RESOURCES INC.

Westcoast Gas Services Inc.

Davis Wire

Sumas, Washington

$0 \quad N / A$

$0 \quad$ N/A

$6 \quad 1.07$

$6 \quad 1.07$

IGI RESOURCES INC.

Westcoast Gas Services Inc.

Draper Farms

Sumas, Washington

$2 \quad 4.17$

$0 \quad$ N/A

$2 \quad 1.07$

$4 \quad 2.82$

IGI RESOURCES INC.

Westcoast Gas Services Inc.

Dyno Polymers

Sumas, Washington

- N/A

$0 \quad$ N/A

$4 \quad 1.07$

$\begin{array}{ll}4 & 1.07\end{array}$

IGI RESOURCES INC.

Westcoast Gas Services Inc. Elanbaas

Sumas, Washington

14.17

$0 \quad$ N/A

$1 \quad 1.07$

$2 \quad 2.71$

IGI RESOURCES INC.

Westcoast Gas Services inc.

Evanite Fiber

Sumas, Washington

$0 \quad$ N/A

- N/A

11

1.07

$11 \quad 1.07$

IGI RESOURCES INC.

Westcoast Gas Services inc.

Fircrest Schools
Sumas, Washington
- N/A

$15 \quad 1.07$

$15 \quad 1.07$ 
SHORT-TERM SALES

YEAR: 1997 Quarter: One

Estimated Volumes (MMCF) \& Prices (\$ / MMBTU)

Importer

Seller

Purchaser / End User

IGI RESOURCES INC.

Westcoast Gas Services Inc.

General Chemical

IGI RESOURCES INC.

Westcoast Gas Services Inc.

Glass Eye

IGI RESOURCES INC.

Westcoast Gas Services Inc. Heath

Sumas, Washington

o N/A

o N/A

$3 \quad 1.07$

1.07

IGI RESOURCES INC

Westcoast Gas Services Inc.

Inland Natural Gas

Sumas, Washington

- N/A

- N/A

108

1.07

108

1.07

IGI RESOURCES INC

Westcoast Gas Services inc. Interox

Sumas, Washington

52

4.17

- N/A

O N/A

52

4.17

IGI RESOURCES INC.

Westcoast Gas Services Inc. J.H. Gypsum

Sumas, Washington

- N/A

- N/A

38

1.07

38

1.07

IGI RESOURCES INC.

Westcoast Gas Services Inc. J.R. Simplot Company

IGI RESOURCES INC.

Westcoast Gas Services Inc.

J.R. Simplot Company

Sumas, Washington

275

4.17

151

2.39

121

1.07

547

2.99

IGI RESOURCES INC.

Westcoast Gas Services inc. Jorgenson Forge

IGI RESOURCES INC.

Westcoast Gas Services Inc. Kings Meat

Sumas, Washington

IGI RESOURCES INC.

Westcoast Gas Services Inc.

Lakeside
$0 \quad$ N/A

O N/A

$5 \quad 1.07$

$5 \quad 1.07$

$0 \quad$ N/A

- N/A

20

1.07

20

1.07

Sumas, Washington
- N/A

- N/A

$\begin{array}{ll}7 & 1.07\end{array}$

7 


\section{SHORT-TERM SALES \\ YEAR: 1997 Quarter: One \\ Estimated Volumes (MMCF) \& Prices (\$ / MMBTU)}

Importer

Seller

Purchaser / End User

IGI RESOURCES INC

Westcoast Gas Services inc.

Lamb Weston

IGI RESOURCES INC.

Westcoast Gas Services Inc.

M.A. Segale

IGI RESOURCES INC

Westcoast Gas Services Inc. MTVHS

IGI RESOURCES INC.

Westcoast Gas Services Inc. Mountain Fuel

Sumas, Washington

IGI RESOURCES INC.

Westcoast Gas Services Inc. Mt. Vemon

Sumas, Washington

IGI RESOURCES INC

Westcoast Gas Services Inc.

Naumes

Sumas, Washington

IGI RESOURCES INC

Westcoast Gas Services Inc. Noram Energy Services

IGI RESOURCES INC.

Westcoast Gas Services Inc. Northstar Energy

IGI RESOURCES INC

Westcoast Gas Services Inc. Northwest Cooperage

IGI RESOURCES INC

Westcoast Gas Services Inc.

Ocean K

Sumas, Washington

o N/A

$0 \quad N / A$

of

January

Vol. Price

February

Vol. Price

March

Vol. Price

Quarterty
Total
Avg.
Vol. Price

Sumas, Washington

42.17

$\begin{array}{ll}73 & 1.07\end{array}$

125

2.35

Sumas, Washington

Sumas, Washington

Sumas, Washington

Sumas, Washington

(

IGI RESOURCES INC

Westcoast Gas Services Inc. Ore-Ida
Sumas, Washington $\begin{array}{ll}7 & 2.39\end{array}$

- N/A

7 


\section{SHORT-TERM SALES \\ YEAR: 1997 Quarter: One \\ Estimated Volumes (MMCF) \& Prices (\$ / MMBTU)}

Importer

Seller

Purchaser/End User

IGI RESOURCES INC.

Westcoast Gas Services inc. Oregon Potato

Sumas, Washington

Sumas, Washington

Westcoast Gas Services inc. Overall Laundry

IGI RESOURCES INC.

Westcoast Gas Services Inc. PABST

IGI RESOURCES INC

Westcoast Gas Services inc. Pendelton

Sumas, Washington

IGI RESOURCES INC.

Westcoast Gas Services Inc. Pierce Transit

IGI RESOURCES INC.

Westcoast Gas Services Inc.

Ponderosa

Sumas, Washington

Sumas, Washington

123

4.17

$39 \quad 2.39$

o N/A

162

3.74

IGI RESOURCES INC.

Westcoast Gas Services Inc.

Rainy River

Sumas, Washington

$2 \quad 4.17$

- N/A

$12 \quad 1.07$

$14 \quad 1.48$

IGI RESOURCES INC.

Westcoast Gas Services inc.

Redhook

Sumas, Washington

- N/A

$0 \quad N / A$

$3 \quad 1.07$

$3 \quad 1.07$

IGI RESOURCES INC.

Westcoast Gas Services Inc.

Roseburg Lumber

Sumas, Washington

- N/A

$20 \quad 2.39$

$3 \quad 1.07$

23

2.20

IGI RESOURCES INC.

Westcoast Gas Services Inc.

Seattle Snohomish

Sumas, Washington
- N/A

- N/A

$6 \quad 1.07$

$6 \quad 1.07$ 


\section{SHORT-TERM SALES \\ YEAR: 1997 Quarter: One \\ Estimated Volumes (MMCF) \& Prices (\$ / MMBTU)}

Importer

Seller

Purchaser / End User

IGI RESOURCES INC.

Westcoast Gas Services Inc.

Smurfit Newsprint Corporation

IGI RESOURCES INC.

Westcoast Gas Services Inc.

Southwest Gas Corporation

IGI RESOURCES INC.

Westcoast Gas Services Inc. Teledyne

IGI RESOURCES INC.

Westcoast Gas Services Inc.

Texaco Gas Marketing Inc.

IGI RESOURCES INC.

Westcoast Gas Services Inc.

Todds Shipyards

IGI RESOURCES INC.

Westcoast Gas Services Inc.

TransOcean Products, Inc.

IGI RESOURCES INC.

Westcoast Gas Services Inc. US Oil \& Refining

IGI RESOURCES INC.

Westcoast Gas Services Inc. Unisea Foods

Sumas, Washington

o N/A

$0 \quad$ N/A

$2 \quad 1.07$

$2 \quad 1.07$

IGI RESOURCES INC.

Westcoast Gas Services Inc.

Valley Medical

Sumas, Washington

$0 \quad$ N/A

O N/A

$5 \quad 1.07$

$5 \quad 1.07$

IGI RESOURCES INC.

Westcoast Gas Services Inc.

WP Natural Gas

Sumas, Washington

$28 \quad 4.17$

O N/A

$54 \quad 1.07$

82

2.13

IGI RESOURCES INC.

Westcoast Gas Services Inc.

Washington Energy Gas Marketing Co.

Sumas, Washington
$17 \quad 4.17$

- N/A

o N/A

17

4.17 


\section{SHORT-TERM SALES \\ YEAR: 1997 Quarter: One \\ Estimated Volumes (MMCF) \& Prices (\$ / MMBTU)}

Importer

Seller

Purchaser / End User

IGI RESOURCES INC.

Westcoast Gas Services inc.

Washington Natural Gas Company

IGI RESOURCES INC.

Westcoast Gas Services Inc.

Washington Water Power Company

IGI RESOURCES INC.

Westcoast Gas Services inc.

Weyerhauser

Sumas, Washington

23

4.17

14

2.39

37

1.07

74

2.27

IGI RESOURCES INC

Westcoast Gas Services Inc.

Willamette

Sumas, Wastington

175

4.17

122

2.39

$2 \quad 1.07$

299

3.42

IGI RESOURCES INC.

Westcoast Gas Services inc.

Woodworth

Sumas, Washington

$0 \quad$ N/A

O N/A

$11 \quad 1.07$

11

1.07

INDECK ENERGY SERVICES, INC.

El Paso Gas Marketing Canada Inc.
Indeck Energy Services of Oswego

Niagara Falls, NY

INLAND PACIFIC ENERGY SERVICES CORP.

Wainoco Oil Corporation

Eastem State Hospital

Sumas, Washington

INLAND PACIFIC ENERGY SERVICES CORP.

Wainoco Oil Corporation

Eastem Washington University

Sumas, Washington

$9 \quad 1.35$

$7 \quad 1.35$

$9 \quad 1.35$

25

1.35

INLAND PACIFIC ENERGY SERVICES CORP.

Wainoco Oil Corporation

Kaiser - Mead

Sumas, Washington

$11 \quad 1.35$

$9 \quad 1.35$

$11 \quad 1.35$

31

1,35

INLAND PACIFIC ENERGY SERVICES CORP.

Wainoco Oil Corporation

Kaiser-T.W

Sumas, Washington

$23 \quad 1.35$

$19 \quad 1.35$

23

1.35

$64 \quad 1.35$

INLAND PACIFIC ENERGY SERVICES CORP

Wainoco Oil Corporation

Lakeland Village

Sumas, Washington
$4 \quad 1.35$

$4 \quad 1.35$

$5 \quad 1.35$

13

1.35 
SHORT-TERM SALES

YEAR: 1997 Quarter: One

Estimated Volumes (MMCF) \& Prices (\$ / MMBTU)

Importer

Seller

Purchaser / End User

INLAND PACIFIC ENERGY SERVICES CORP.

Wainoco Oil Corporation

Lane Mountain Silica

Sumas, Washington

$7 \quad 1.35$

$6 \quad 1.35$

$8 \quad 1.35$

$20 \quad 1.35$

INLAND PACIFIC ENERGY SERVICES CORP.

Wainoco Oil Corporation

Travis Pattem

Sumas, Washington

$8 \quad 1.35$

$7 \quad 1.35$

$9 \quad 1.35$

$24 \quad 1.35$

INLAND PACIFIC ENERGY SERVICES CORP.

Wainoco Oil Corporation

Washington Potato Company

Sumas, Washington

INLAND PACIFIC ENERGY SERVICES LTD.

Inland Pacific Energy Services Ltd.

Aquila Energy Marketing

Sumas, Washington

$3 \quad 3.42$

$0 \quad$ N/A

$0 \quad$ N/A

$3 \quad 3.42$

INLAND PACIFIC ENERGY SERVICES LTD. Inland Pacific Energy Services Ltd.

Cabot Oil

Sumas, Washington

22

2.38

3

2.15

$91 \quad 1.09$

116

1.36

INLAND PACIFIC ENERGY SERVICES LTD. Inland Pacific Energy Services Ltd.

Cascade Natural Gas

Sumas, Washington

INLAND PACIFIC ENERGY SERVICES LTD.

inland Pacific Energy Services Ltd.

Enron Gas Marketing

Sumas, Washington

$2 \quad 2.55$

126

1.84

155

1.84

283

1.85

INLAND PACIFIC ENERGY SERVICES LTD.

Intand Pacific Energy Services Ltd.

Inland Pacific Energy Services Corp

Sumas, Washington

605

3.62

535

2.19

606

1.11

1746

2.31

INLAND PACIFIC ENERGY SERVICES LTD. Inland Pacific Energy Services Ltd.

Northwest Natural Gas Company

Sumas, Washington

3.27

- N/A

- N/A

70

3.27

INLAND PACIFIC ENERGY SERVICES LTD. Inland Pacific Energy Services Ltd.

PanEnergy Trading \& Marketing Svcs.

Sumas, Washington

INLAND PACIFIC ENERGY SERVICES LTD.

Inland Pacific Energy Services Ltd.

Questar Energy Trading Co.

Sumas, Washington
$5 \quad 2.14$

48

1.26

$3 \quad 1.12$

56

1.33
$0 \quad N / A$

O N/A
$3 \quad 1.17$

$\begin{array}{ll}3 & 1.17\end{array}$ 
SHORT-TERM SALES

YEAR: 1997 Quarter: One

Estimated Volumes (MMCF) \& Prices (\$ / MMBTU)
Importer

Seller

Purchaser / End User

INLAND PACIFIC ENERGY SERVICES LTD.

Inland Pacific Energy Services Litd.

Sierra Pacific Power Company

INLAND PACIFIC ENERGY SERVICES LTD.

Inland Pacific Energy Services Ltd.

Southwest Gas Corporation

INTALCO ALUMINUM CORPORATION

CanWest Gas Supply Inc.

Intalco Aluminum Corporation

Point

of

Entry

Sumas, Washington

103

4.17

Sumas, Washington

$5 \quad 3.63$

$8 \quad 1.08$

$3 \quad 1.26$

15

1.94

Sumas, Washington

$48 \quad 1.51$

$46 \quad 1.46$

$46 \quad 1.31$

140

1.43

INTERENERGY CORPORATION

Husky Gas Marketing Inc.

Interenergy Corporation

INTERENEAGY CORPORATION

Ocelot Energy, Inc.

Interenergy Corporation

Portal, ND

4.06

280

2.36

$0 \quad$ N/A

590

3.25

INTERENERGY CORPORATION

Poco Marketing Ltd.

Interenergy Corporation

Portal, ND

o N/A

o N/A

$310 \quad 1.28$

310

1.28

INTERENERGY CORPORATION

TransCanada Gas Services Limited

Interenergy Corporation

Babb, Montana

$14 \quad 1.86$

D N/A

$71 \quad 1.23$

85

1.34

IROQUOIS ENERGY MANAGEMENT, INC.

Renaissance Energy Ltd.

SUNY - N.Y.

Niagara Falls, NY

$349 \quad 3.25$

339

2.70

280

2.40

968

2.81

JONAN GAS MARKETING

Jonan Gas Marketing Ltd.

Mock Resources

Eastport, Idaho

$6 \quad 3.32$

0

N/A

0

N/A

$6 \quad 3.32$

JONAN GAS MARKETING

Jonan Gas Marketing Ltd.

Pacific Gas \& Electric Company

Eastport, Idaho

$5 \quad 3.32$

0

N/A

0

N/A

5

3.32

KCS ENERGY MARKETING, INC.

Cibola Canada Energy Marketing Co.

Milford Power, L.P.
Niagara Falls, NY

188

2.10

168

2.15

188

2.06

544

2.10 
SHORT-TERM SALES

YEAR: 1997 Quarter: One

Estimated Volumes (MMCF) \& Prices (\$ / MMBTU)
Importer

Seller

Purchaser / End User

KCS ENERGY MARKETING, INC.

Renaissance Energy Ltd.

Various NY State Markets

KCS ENERGY MARKETING, INC.

Renaissance Energy Ltd.

Various NY State Markets

KCS ENERGY MARKETING, INC.

Renaissance Energy Ltd.

Various NY State Markets

KCS ENERGY MARKETING, INC.

Renaissance Energy Ltd.

Various NY State Markets

KCS ENERGY MARKETING, INC SEMCO

Various NY State Markets

Grand island, NY

$0 \quad$ N/A

15

2.72

January

Vol. Price

February

Vol. Price

March

Vol. Price

Quarterly

Total

Avg.

Vol. Price

Grand Island, NY

$53 \quad 5.14$

$30 \quad 3.65$

$9 \quad 2.54$

91

4.40

Niagara Falls, NY

$17 \quad 6.82$

$0 \quad$ N/A

$0 \quad$ N/A

17

6.82

Noyes, Minnesota

$440 \quad 2.15$

$173 \quad 2.58$

184

1.59

796

2.11

KCS ENERGY MARKETING, INC.

SEMCO

Various NY State Markets

Waddington, NY

14.90

$0 \quad N / A$

$0 \quad N / A$

1

4.90

KOCH ENERGY TRADING, INC

PEMEX

Koch Energy Trading

Hidalgo, Texas

$0 \quad N / A$

$11 \quad 2.29$

$40 \quad 1.76$

51

1.87

KOCH GAS SERVICES COMPANY

Koch Gas Services Canada

Koch Gas Services Company

LG\&E NATURAL MARKETING INC.

LG\&E Natural Marketing Canada Inc.

AIG Trading Corporation

Niagara Falls, NY

140

3.55

304

2.27

599

3.14

LG\&E NATURAL MARKETING INC.

LG\&E Natural Marketing Canada Inc.

Aquila Energy Marketing

Niagara Falls, NY

$0 \quad$ N/A

140

3.41

O N/A

140

3.41

LG\&E NATURAL MARKETING INC.

LG\&E Natural Marketing Canada Inc.

Enron Gas Marketing
Niagara Falls, NY
$0 \quad$ N/A

14

3.39
- N/A

142

3.39 
SHORT-TERM SALES

YEAR: 1997 Quarter: One

Estimated Volumes (MMCF) \& Prices (\$ / MMBTU)
Importer

Seller

Purchaser / End User

LG\&E NATURAL MARKETING INC.

LG\&E Natural Marketing Canada Inc.

Enserch Energy Services, Inc.

LG\&E NATURAL MARKETING INC.

LG\&E Natural Marketing Canada inc.

Michigan Consolidated Gas Company

LG\&E NATURAL MARKETING INC.

LG\&E Natural Marketing Canada Inc.

SEMCO Energy Services

Point

of

Entry

Grand Island, NY

St. Clair, Michigan

Grand Island, NY

$310 \quad 4.71$

- N/A

310

2.27

620

3.49

LG\&E NATURAL MARKETING INC.

LG\&E Natural Marketing Canada Inc.

Wascana Energy Marketing

Niagara Falls, NY

$311 \quad 5.31$

$0 \quad$ N/A

N/A

311

5.31

MASSPOWER

ProGas Limited

Orchard Gas

Niagara Falls, NY

O N/A

2.09

183

2.15

247

MURPHY GAS GATHERING

Murphy Oil Company Ltd.

Murphy Gas Gathening

Noyes, Minnesota

$101 \quad 4.07$

$91 \cdot 2.89$

10

1.46

293

2.80

N.Y.STATE ELECTRIC \& GAS CORPORATION

Shell Canada Limited

Various NY State Markets

Champlain, NY

$57 \quad 3.68$

$63 \quad 2.58$

84

2.19

205

2.73

NATIONAL FUEL GAS DISTRIBUTION

CNG Enercy

Various NYS State Markets

Niagara Falls, NY

$0 \quad N / A$

118

2.43

$241 \quad 2.09$

359

2.20

NATIONAL FUEL GAS DISTRIBUTION Howard Energy Company, Inc.

Various NY State Markets

Niagara Falls, NY

$266 \quad 3.00$

174

3.17

124

2.05

564

2.84

NATIONAL FUEL GAS DISTRIBUTION

KCS Energy Management Services

Various NY State Markets

Niagara Falls, NY

$30 \quad 3.50$

- N/A

o N/A

30

3.50

NATIONAL FUEL GAS DISTRIBUTION

Renaissance Energy Ltd.

Various NY State Markets
Niagara Falls, NY
$27 \quad 2.22$

20

2.09

122

3.07 
SHORT-TERM SALES

YEAR: 1997 Quarter: One

Estimated Volumes (MMCF) \& Prices ( $\$$ / MMBTU)
Importer

Seller

Purchaser / End User

NATIONAL FUEL GAS DISTRIBUTION SEMCO

Various NY State Markets

NATIONAL FUEL GAS DISTRIBUTION SEMCO

Various NY State Markets

Point

of

Entry

Grand Island, NY

$5 \quad 3.15$

O N/A

O N/A

5

3.15

Niagara Falls, NY

O N/A

$13 \quad 2.11$

$15 \quad 2.10$

29

2.10

NATIONAL FUEL GAS DISTRIBUTION Stamp Gas Inc.

Various NY State Markets

Niagara Falls, NY

$10 \quad 3.23$

- N/A

- N/A

10

3.23

NATIONAL FUEL GAS DISTRIBUTION

Temco

Various NY State Markets

Niagara Falls, NY

- N/A

$3 \quad 2.07$

- N/A

$3 \quad 2.07$

NATIONAL FUEL GAS DISTRIBUTION Tenneco

Various NY State Markets

Grand Island, NY

$18 \quad 3.05$

O N/A

- N/A

18

3.05

NATIONAL FUEL GAS DISTRIBUTION Texaco Gas Marketing Inc.

Various NY State Markets

NATIONAL FUEL GAS DISTRIBUTION TransCanada Gas Services Limited

Various NY State Markets

Grand Istand, NY

- N/A

10

2.12

- N/A

10

2.12

NATIONAL FUEL GAS DISTRIBUTION TransCanada Gas Services Limited Various NY State Markets

Niagara Falls, NY

$0 \quad$ N/A

$0 \quad$ N/A

$9 \quad 2.12$

9

2.12

NATIONAL FUEL GAS DISTRIBUTION Union Oil Company of Califomia

Various NY State Markets

Niagara Falls, NY

$10 \quad 3.15$

12.08

- N/A

11

3.04

NATIONAL FUEL GAS DISTRIBUTION Wascana Energy Marketing Inc.

Various NY State Markets

Grand Island, NY

$118 \quad 3.85$

O N/A

N/A

118

3.85

NATIONAL FUEL GAS DISTRIBUTION Wascana Energy Marketing Inc.

Various NY State Markets
Niagara Falls, NY
$0 \quad$ N/A

2.34

$29 \quad 2.07$

31

2.08 
SHORT-TERM SALES

YEAR: 1997 Quarter: One

Estimated Volumes (MMCF) \& Prices (\$ / MMBTU)
Importer

Seller

Purchaser / End User

NATIONAL FUEL GAS DISTRIBUTION

Western Gas Resources

Various NY State Markets

NATIONAL FUEL RESOURCES, INC.

Coastal Gas Marketing, Canada

Various NY State Markets

NATIONAL FUEL RESOURCES, INC.

Noram Energy Services

Various NY State Markets

NATIONAL FUEL RESOURCES, INC.

Renaissance Energy Ltd.

Various NY State Markets

NATIONAL FUEL RESOURCES, INC.

Renaissance Energy Ltd.

Various NY State Markets

NATIONAL FUEL RESOURCES, INC. SEMCO

Various NY State Markets

Niagara Falls, NY

$5 \quad 4.00$

- N/A

o N/A

5. 4.00

NATIONAL FUEL RESOURCES, INC.

Stampeder Energy

Various NY State Markets

Grand Island, NY

$9 \quad 5.25$

- N/A

$6 \quad 2.05$

15

3.87

NATIONAL FUEL RESOURCES, INC.

Stampeder Energy

Various NY State Markets

Niagara Falls, NY

205

5.09

138

3.36

$146 \quad 2.03$

488

3.69

NATIONAL FUEL RESOURCES, INC.

Wascana Energy Marketing Inc.

Various NY State Markets

Grand Island, NY

O N/A

0

N/A

$7 \quad 2.04$

$7 \quad 2.04$

NATIONAL FUEL RESOURCES, INC. Wascana Energy Marketing Inc.

Various NY State Markets

Niagara Falls, NY

$20 \quad 4.37$

$5 \quad 3.10$

$140 \cdot 2.04$

165

2.35

NATIONAL FUEL RESOURCES, INC.

Westcoast Gas Services Inc.

Various NY State Markets

Niagara Falls, NY
366

4.89
296

3.18 


\section{SHORT-TERM SALES \\ YEAR: 1997 Quarter: One \\ Estimated Volumes (MMCF) \& Prices (\$ / MMBTU)}

Importer

Seller

Purchaser / End User

NATIONAL FUEL RESOURCES, INC. Williams Energy Services Company Various NY State Markets

NATIONAL STEEL CORPORATION Direct Energy Marketing Limited National Steel Corporation

NATIONAL STEEL CORPORATION Kimball Energy Corporation National Steel Corporation

NATIONAL STEEL CORPORATION PanEnergy Marketing Canada

National Steel Corporation

NATURAL GAS CLEARINGHOUSE PEMEX

Natural Gas Clearinghouse

NEW ENGLAND POWER COMPANY

Canadian Natural Resources Limited New England Power Company

NMU GAS PURCHASING INC.

ProGas Limited

NMU Gas Purchasing Inc.

NMU GAS PURCHASING INC.

TransCanada Gas Services Limited NMU Gas Purchasing Inc.

Warroad, Minnesota

20

3.54

15

2.11

14

1.18

49

2.42

NORAM ENERGY SERVICES, INC. PEMEX

Noram Energy Services

NORSTAR ENERGY, L.P.

TransCanada Gas Services Limited Medina Power Company

NORTH AMERICAN ENERGY, INC.

Various Suppliers

North American Energy inc.

Point

Entry

January

Vol. Price

Vol. Price

Vol. Price

Quarterty

Vol. Prgice

Niagara Falls, NY

$\begin{array}{llll}0 & \text { N/A } & 12 \quad 3.06\end{array}$

- N/A

12

3.06

Detroit, Michigan

- N/A

$0 \quad$ N/A

15

2.36

15

2.36

Detroit, Michigan

- N/A

$5 \quad 2.85$

- N/A

5

2.85

Detroit, Michigan

35

292

.05

2.04

901

2.03

Waddington, NY

$230 \quad 1.65$

- N/A

0

N/A

230

1.65

Hidaigo, Texas

$28 \quad 3.02$

110

2.73

1.80

195

2.50

Niagara Falls, NY

78

4.49

68

2.47

78

2.11

223

3.05

Grand Island, NY $\begin{array}{llll}19 & 4.04 & 16 & 3.68\end{array}$

15

2.70

49

3.52 


\section{SHORT-TERM SALES \\ YEAR: 1997 Quarter: One \\ Estimated Volumes (MMCF) \& Prices (\$ / MMBTU)}

Importer

Seller

Purchaser / End User

NORTH AMERICAN ENERGY, INC.

Various Suppliers

North Amencan Energy inc.

\section{Point \\ of \\ Entry}

January
Vol. Price
February

Vol. Price
March

Vol. Price

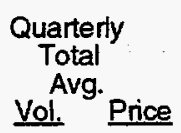

Vol. Price

NORTH AMERICAN RESOURCES COMPANY

Altana Exploration

Various CO State Markets

Babb, Montana

- N/A

- N/A

$92 \quad 1.80$

$92 \quad 1.80$

NORTH CANADIAN MARKETING CORPORATION

Norcen Energy Resources

Cibola Corporation

Port of Morgan, MT

$309 \quad 2.83$

28

1.92

314

1.18

905

1.97

NORTH CANADIAN MARKETING CORPORATION

Norcen Energy Resources

Coastal Gas Marketing Company

Port of Morgan, MT

$618 \quad 2.83$

$559 \quad 1.92$

619

1.18

1795

1.98

NORTH CANADIAN MARKETING CORPORATION

Norcen Energy Resources

Michigan Consolidated Gas Company

Noyes, Minnesota

221

3.19

199

3.19

220

3.19

640

3.19

NORTH CANADIAN MARKETING CORPORATION

Various Suppliers

AIG Trading Corporation

Eastport, Idaho

$706 \quad 1.54$

$606 \quad 1.25$

$660 \quad 1.07$

1972

1.29

NORTH CANADIAN MARKETING CORPORATION

Various Suppliers

Coastal Gas Marketing Company

Eastport, Idaho

$265 \quad 1.54$

$227 \quad 1.25$

23

1.07

729

1.30

NORTH CANADIAN MARKETING CORPORATION

Various Suppliers

Cook Inlet

Eastport, Idaho

- N/A

$3 \quad 1.25$

$0 \quad$ N/A

3

1.25

NORTH CANADIAN MARKETING CORPORATION

Various Suppliers

Coral Energy Resources

Eastport, Idaho

$176 \quad 1.54$

151

1.25

158

1.07

$486 \quad 1.30$

NORTH CANADIAN MARKETING CORPORATION

Various Suppliers

Enron Capital \& Trade Resources Eastport, Idaho

O N/A

$0 \quad$ N/A

163

1.07

163

1.07

NORTH CANADIAN MARKETING CORPORATION

Various Suppliers

Enron Gas Marketing

Eastport, Idaho

$\begin{array}{llll}267 & 1.54 & 278 & 1.25\end{array}$

$0 \quad$ N/A

545

1.39 
SHORT-TERM SALES

YEAR: 1997 Quarter: One

Estimated Volumes (MMCF) \& Prices ( $\$$ / MMBTU)

Importer

Seller

Purchaser / End User

Point

Point
of

Entry

\author{
January \\ Vol. Price \\ February
Vol. Price
}

Quarterty
Total

March

Vol. Price

NORTH CANADIAN MARKETING CORPORATION

Various Suppliers

Equitable Resources Marketing Co.

Eastport, Idaho

$176 \quad 1.54$

$39 \quad 1.25$

$83 \quad 1.07$

298

1.37

NORTH CANADIAN MARKETING CORPORATION

Various Suppliers

Natural Gas Clearinghouse

Eastport, Idaho

336

1.54

32

1.25

$332 \quad 1.07$

989

1.29

NORTH CANADIAN MARKETING CORPORATION

Various Suppliers

PanEnergy Trading \& Marketing Svcs. Eastport Idaho

681

1.54

832

1.25

$914 \quad 1.07$

2427

1.26

NORTH CANADIAN MARKETING CORPORATION

Various Suppliers

TransCanada Gas Services

Eastport, Idaho

18

1.54

$204 \quad 1.25$

$162 \quad 1.07$

$547 \quad 1.29$

NORTH CANADIAN MARKETING CORPORATION

Various Suppliers

Washington Water Power Company

Eastport, Idaho

$459 \quad 1.54$

39

1.25

405

1258

1.30

NORTH CANADIAN MARKETING CORPORATION

Various Suppliers

Wickford Energy Marketing inc.

Eastport, Idaho

- N/A

- N/A

$31 \quad 1.07$

$31 \quad 1.07$

NUMAC ENERGY (U.S.) INC.

Numac Energy

Enron Capital \& Trade Resources

Port of Morgan, MT

300

2.32

280

2.10

$310 \quad 1.58$

890

1.99

NUMAC ENERGY (U.S.) INC.

Numac Energy

National Gas \& Electric

Eastport, Idaho

156

3.71

141

2.07

156

1.21

454

2.34

NUMAC ENERGY (U.S.) INC.

Numac Energy

Natural Gas Clearinghouse

Port of Morgan, MT

155

3.60

14

2.31

155

1.12

450

2.34

NUMAC ENERGY (U.S.) INC.

Numac Energy

TransCanada Gas Services

Eastport, Idaho

$155 \quad 2.73$

14

2.06

$155 \quad 1.22$

450

2.00

NUMAC ENERGY (U.S.) INC.

Numac Energy

Wascana Energy Marketing

Port of Morgan, MT

$152 \quad 3.59$

140

2.31

155

1.12

447

2.33 
SHORT-TERM SALES

YEAR: 1997 Quarter: One

Estimated Volumes (MMCF) \& Prices (\$ / MMBTU)
Importer

Seller

Purchaser / End User

ONYX GAS MARKETING COMPANY, L.C.

\section{PEMEX}

Onyx Gas Marketing Company

PAN-ALBERTA GAS (U.S.), INC.

Various Suppliers

Amoco Trading

PAN-ALBERTA GAS (U.S.), INC.

Various Suppliers

Aquila Energy Marketing

PAN-ALBERTA GAS (U.S.), INC.

Various Suppliers

Cibola Corporation

PAN-ALBERTA GAS (U.S.), INC.

Various Suppliers

Cook Inlet

Eastport, Idaho

Sumas, Washington

$148 \quad 1.64$

$134 \cdot 1.62$

148

1.62

430

1.63

PAN-ALBERTA GAS (U.S.), INC.

Various Suppliers

Minnegasco

PAN-ALBERTA GAS (U.S.), INC

Various Suppliers

Natural Gas Clearinghouse

PAN-ALBERTA GAS (U.S.), INC

Various Suppliers

Natural Gas Clearinghouse

PAN-ALBERTA GAS (U.S.), INC.

Various Suppliers

Pacific Gas \& Electric Company

PAN-ALBERTA GAS (U.S.), INC.

Various Suppliers

PanEnergy †rading \& Marketing Svcs.
Eastport, Idaho

1809

1.73

Port of Morgan, MT

$188 \quad 3.78$

O N/A

O N/A

188

3.78

Sumas, Washington

$263 \quad 1.64$

23

1.62

26

1.62

765

1.63

Eastport, Idaho

$\begin{array}{llll}25 & 1.73 & 88 & 1.58\end{array}$

- N/A

113

1.61

Eastport, Idaho

$90 \quad 1.58$

o N/A

90

1.58 
SHORT-TERM SALES

YEAR: 1997 Quarter: One

Estimated Volumes (MMCF) \& Prices (\$ / MMBTU)
Importer

Seller

Purchaser / End User

PAN-ALBERTA GAS (U.S.), INC.

Various Suppliers

PanEnergy trading \& Marketing Svcs.

PAN-ALBERTA GAS (U.S.), INC.

Various Suppliers

Poco Petroleum

PAN-ALBERTA GAS (U.S.), INC

Various Suppliers

Sierra Pacific Power Company

PAN-ALBERTA GAS (U.S.), INC

Various Suppliers

Tenaska Marketing Ventures

PAN-ALBERTA GAS (U.S.), INC.

Various Suppliers

U.S. Gas Transportation Inc.

PAN-ALBERTA GAS (U.S.), INC.

Various Suppliers

U.S. Gas Transportation Inc.

PAN-ALBERTA GAS (U.S.), INC.

Various Suppliers

Utilicorp United

Port of Morgan, MT

- N/A

O N/A

$4 \quad 1.38$

$4 \quad 1.38$

PAN-ALBERTA GAS (U.S.), INC.

Various Suppliers

Valero Gas Marketing

PAN-ALBERTA GAS (U.S.), INC.

Various Suppliers

Westcoast Gas Services (U.S.A.) Inc

Port of Morgan, MT

$26 \quad 3.78$

$0 \quad$ N/A

$0 \quad$ N/A

26

3.78

PANCANADIAN PETROLEUM COMPANY PanCanadian Petroleum Ltd.

Exxon Corporation

Eastport, Idaho

$880 \quad 3.64$

522

1.99

62

0.98

2024

2.40

PANCANADIAN PETROLEUM COMPANY

PanCanadian Petroleum Ltd.

National Gas \& Electric
Eastport, Idaho 


\section{SHORT-TERM SALES \\ YEAR: 1997 Quarter: One \\ Estimated Volumes (MMCF) \& Prices (\$ / MMBTU)}

Importer

Seller

Purchaser/End User

PANCANADIAN PETROLEUM COMPANY

PanCanadian Petroleum Ltd.

National Gas \& Electric

PANCANADIAN PETROLEUM COMPANY

PanCanadian Petroleum Ltd.

Northem Califomia Power Agency

Eastport, Idaho

Lake Charles, LA

PANENERGY LNG SALES, INC.

Sonatrading

PanEnergy Trading \& Marketing Svcs.
Point

of

Port of Morgan, MT

$1912 \quad 3.64$

174

February

Vol. Price

January

March

Vol. Price

Quarterly
Total
Avg.
Vol. Price

\section{PANENERGY TRADING AND MARKET SERVICES L.L.C.}

\section{PEMEX}

PanEnergy Trading \& Marketing Svcs.

Hidalgo, Texas

$89 \quad 3.08$

O N/A

$71 \quad 1.77$

160

2.50

PANENERGY TRADING AND MARKET SERVICES L.L.C.

PanEnergy Marketing Canada

PanEnergy Trading \& Marketing Svcs. Eastport, ldaho

$1750 \quad 3.74$

3.74

$1562 \quad 2.32$

189

1.19

5203

2.39

PANENERGY TRADING AND MARKET SERVICES L.L.C.

PanEnergy Marketing Canada

PanEnengy Trading \& Marketing Svas.

Grand Island, NY

$94 \quad 4.65$

$0 \quad$ N/A

$0 \quad N / A$

$94 \quad 4.65$

PANENERGY TRADING AND MARKET SERVICES L.L.C.

PanEnergy Marketing Canada

PanEnergy Trading \& Marketing Svcs. Noyes, Minnesota

$702 \quad 4.11$

$664 \quad 2.56$

$509 \quad 1.34$

1875

2.81

PANENERGY TRADING AND MARKET SERVICES L.L.C.

PanEnergy Marketing Canada

PanEnergy Trading \& Marketing Svcs.

St. Clair, Michigan

$85 \quad 3.50$

O N/A

O N/A

85

3.50

PANENERGY TRADING AND MARKET SERVICES L.L.C.

PanEnergy Marketing Canada

PanEnergy Trading \& Marketing Svcs.

Sumas, Washington

$6312 \quad 4.15$

$3673 \quad 2.37$

$5022 \quad 1.05$

15007

2.68

PANENERGY TRADING AND MARKET SERVICES L.L.C.

PanEnergy Marketing Canada

PanEnergy Trading \& Marketing Svcs. Waddington, NY

$620 \quad 4.79$

$520 \quad 3.41$

$655 \quad 2.07$

$1794 \quad 3.40$

PAWTUCKET POWER ASSOCIATES L.P.

Pawtucket Power Associates

CNG Energy Services

Waddington, NY

O N/A

$5 \quad 1.24$

$0 \quad$ N/A

5

1.24 


\section{SHORT-TERM SALES \\ YEAR: 1997 Quarter: One \\ Estimated Volumes (MMCF) \& Prices (\$ / MMBTU)}

Importer

Seller

Purchaser/End User

Point

of

January

Vol. Price

February

Vol. Price

March

Vol. Price

Quarterty

Total

Entn

Waddington, NY

$1 \quad 2.33$

O N/A

O N/A

12.33

Pawtucket Power Associates

CNG Gas Services

PAWTUCKET POWER ASSOCIATES L.P.

Pawtucket Power Associates

CoEnergy Trading Company

Waddington, NY

$5 \quad 2.33$

$13 \quad 1.24$

$5 \quad 1.26$

22

1.49

PAWTUCKET POWER ASSOCIATES L.P.

Pawtucket Power Associates

Northeast Utilities

Waddington, NY

$0 \quad$ N/A

O N/A

$84 \quad 1.25$

$84 \quad 1.25$

PAWTUCKET POWER ASSOCIATES L.P.

Pawhucket Power Associates

PanEnergy Gas Services, Inc.

Waddington, NY

- N/A

O N/A

$3 \quad 1.25$

$3 \quad 1.25$

PETRO-CANADA HYDROCARBONS, INC. Petro-Canada Hydrocarbons Inc.

Arco Products Company

Sumas, Washington

$\begin{array}{ll}70 & 2.87\end{array}$

$10 \quad 2.07$

$0 \quad$ N/A

80

2.77

PETRO-CANADA HYDROCARBONS, INC. Petro-Canada Hydrocarbons inc.

Barret Fuels

Sumas, Washington

$0 \quad$ N/A

$0 \quad$ N/A

$90 \quad 1.05$

90

1.05

PETRO-CANADA HYDROCARBONS, INC.

Petro-Canada Hydrocarbons inc.

Coastal Gas Marketing Company

Eastport, Idaho

$0 \quad$ N/A

68

1.88

$746 \quad 1.41$

1432

1.64

PETRO-CANADA HYDROCARBONS, INC.

Petro-Canada Hydrocarbons inc.

Coastal Gas Marketing Company

Sumas, Washington

$576 \quad 2.39$

O N/A

o. N/A

576

2.39

PETRO-CANADA HYDROCARBONS, INC Petro-Canada Hydrocarbons Inc.

|G| Resources

Sumas, Washington

272

4.33

260

2.41

3341.08

866

2.50

PETRO-CANADA HYDROCARBONS, INC Petro-Canada Hydrocarbons Inc.

Noram Energy Services

Sumas, Washington

$7 \quad 2.85$

$0 \quad$ N/A

$0 \quad$ N/A

$7 \quad 2.85$

PETRO-CANADA HYDROCARBONS, INC

Petro-Canada Hydrocarbons Inc.

Questar Energy Trading Co.

Sumas, Washington

- N/A

$16 \quad 1.44$

- N/A

$16 \quad 1.44$ 


\section{SHORT-TERM SALES \\ YEAR: 1997 Quarter: One \\ Estimated Volumes (MMCF) \& Prices (\$ / MMBTU)}

Importer

Seller

Purchaser/End User

PETRO-CANADA HYDROCARBONS, INC.

Petro-Canada Hydrocarbons Inc.

Southwest Gas Corporation

PETRO-CANADA HYDROCARBONS, INC.

Petro-Canada Hydrocarbons Inc.

Washington Natural Gas Company

PETRO-CANADA HYDROCARBONS, INC.

Petro-Canada Hydrocarbons Inc.

Washington Water Power Company

Point

of

Entry

Sumas, Washington

132

2.79

$127 \quad 1.63$

$84 \quad 1.20$

343

1.97

Sumas, Washington

$299 \quad 3.02$

268

2.10

$270 \quad 0.96$

838

2.06

PETRO-CANADA HYDROCARBONS, INC.

Petro-Canada Hydrocarbons inc.

Washington Water Power Company

PETRO-CANADA HYDROCARBONS, INC.

Petro-Canada Hydrocarbons Inc.

Western Gas Resources

Sumas, Washington

$10 \quad 2.47$

$0 \quad$ N/A

$0 \quad$ N/A

10

2.47

PG\&E GAS SUPPLY BUSINESS UNIT

AEC Oil \& Gas Company

PG\&E Gas Supply Business Unit

Eastport, Idaho

$\begin{array}{ll}68 & 2.31\end{array}$

$1229 \quad 1.06$

$19 \quad 1.26$

1317

1.13

Eastport, Idaho

$906 \quad 1.70$

o N/A

1042

1.26

1948

1.46

PG\&E GAS SUPPLY BUSINESS UNIT Amoco Canada Petroleum Company Ltd. PG\&E Gas Supply Business Unit

Eastport, Idaho

$1381 \quad 1.67$

204

1.42

2437

1.30

5866

1.43

PG\&E GAS SUPPLY BUSINESS UNIT

Aquila Canada Corporation

PG\&E Gas Supply Business Unit

Eastport, Idaho

$340 \quad 2.10$

80

1.42

50

1.12

1194

1.60

PG\&E GAS SUPPLY BUSINESS UNIT B.C. Gas Inc.

PG\&E Gas Supply Business Unit

Eastport, Idaho

O N/A

- N/A

1.18

170

1.18

PG\&E GAS SUPPLY BUSINESS UNIT

Canadian 88 Energy

PG\&E Gas Supply Business Unit

Eastport, ldaho
$5 \quad 1.61$

- N/A

$40 \quad 1.08$

44

1.14 


\section{SHORT-TERM SALES \\ YEAR: 1997 Quarter: One \\ Estimated Volumes (MMCF) \& Prices (\$/MMBTU)}

Importer

Seller

Purchaser / End User

PG\&E GAS SUPPLY BUSINESS UNIT

Canadian Hunter Exploration Ltd.

PG\&E Gas Supply Business Unit

PG\&E GAS SUPPLY BUSINESS UNIT

Chevron Canada Resources

PG\&E Gas Supply Business Unit

PG\&E GAS SUPPLY BUSINESS UNIT Cibola Canada Energy Marketing Co.

PG\&E Gas Supply Business Unit

Eastport, Idaho

$\begin{array}{llll}0 & \text { N/A } & 219 & 1.87\end{array}$

$0 \quad$ N/A

219

1.87

PG\&E GAS SUPPLY BUSINESS UNIT Cook Inlet Energy Supply

PG\&E Gas Supply Business Unit

PG\&E GAS SUPPLY BUSINESS UNIT Direct Energy Marketing Limited

PG\&E Gas Supply Business Unit

PG\&E GAS SUPPLY BUSINESS UNIT EI Paso Energy Markeing Canada

PG\&E Gas Supply Business Unit

PG\&E GAS SUPPLY BUSINESS UNIT EI Paso Gas Marketing Canada Inc.

PG\&E Gas Supply Business Unit

PG\&E GAS SUPPLY BUSINESS UNIT Energy Source Canada, Inc.

PG\&E Gas Supply Business Unit

Eastport, Idaho

$66 \quad 2.22$

$546 \quad 1.99$

O N/A

612

2.01

PG\&E GAS SUPPLY BUSINESS UNIT Enron Capital \& Trade Resources Can PG\&E Gas Supply Business Unit

Eastport, Idaho

$336 \quad 1.70$

1093

1.72

54

1.20

1974

1.57

PG\&E GAS SUPPLY BUSINESS UNIT Ensource

PG\&E Gas Supply Business Unit

Eastport, Idaho

$755 \quad 1.67$

172

2.20

$302 \quad 1.30$

2783

1.96

PG\&E GAS SUPPLY BUSINESS UNIT Equitable Resources Marketing Canad PG\&E Gas Supply Business Unit
Eastport, Idaho
$563 \quad 1.07$

$49 \quad 1.81$

1025

1.15

1637

1.14 


\section{SHORT-TERM SALES \\ YEAR: 1997 Quarter: One \\ Estimated Volumes (MMCF) \& Prices (\$ / MMBTU)}

Importer

Seller
Punchaser / End User

PG\&E GAS SUPPLY BUSINESS UNIT IGI Resources

PG\&E Gas Supply Business Unit

Eastport, Idaho

PG\&E GAS SUPPLY BUSINESS UNIT $J$. Aron \& Company

PG\&E Gas Supply Business Unit

PG\&E GAS SUPPLY BUSINESS UNIT Koch Gas Services Canada

PG\&E Gas Supply Business Unit

PG\&E GAS SUPPLY BUSINESS UNIT LG\&E Natural Marketing Canada Inc. PG\&E Gas Supply Business Unit

PG\&E GAS SUPPLY BUSINESS UNIT Louis Dreyfus Energy Corporation PG\&E Gas Supply Business Unit

PG\&E GAS SUPPLY BUSINESS UNIT Northstar Energy Comporation

PG\&E Gas Supply Business Unit

Eastport, Idaho

$588 \quad 1.83$

$589 \quad 1.54$

$425 \quad 1.20$

$1602 \quad 1.56$

PG\&E GAS SUPPLY BUSINESS UNIT Novagas Clearinghouse Ltd.

PG\&E Gas Supply Business Unit

Eastport, Idaho

$1450 \quad 1.59$

$178 \quad 2.05$

$648 \quad 1.20$

$2277 \quad 1.52$

PG\&E GAS SUPPLY BUSINESS UNIT

Pan-Alberta Gas Ltd.

PG\&E Gas Supply Business Unit

Eastport, Idaho

$767 \quad 1.67$

693

2.12

903

1.26

2363

1.65

PG\&E GAS SUPPLY BUSINESS UNIT

PanCanadian Petroleum Lid.

PG\&E Gas Supply Business Unit

Eastport, Idaho

O N/A

$691 \quad 1.08$

$146 \quad 1.14$

837. $\quad 1.09$

PG\&E GAS SUPPLY BUSINESS UNIT Petro-Canada Hydrocarbons Inc.

PG\&E Gas Supply Business Unit

Eastport, Idaho

$614 \quad 1.68$

703

2.11

$302 \quad 1.30$

$1619 \quad 1.80$

PG\&E GAS SUPPLY BUSINESS UNIT ProGas Limited

PG\&E Gas Supply Business Unit
Eastport, Idaho $\begin{array}{llll}388 & 1.68 & 183 & 1.52\end{array}$

495

1.13

1066

1.40
Total Vol.

581

1.72

.41

.09

Quarterly 
SHORT-TERM SALES

YEAR: 1997 Quarter: One

Estimated Volumes (MMCF) \& Prices (\$ / MMBTU)
Importer

Seller

Purchaser / End User

PG\&E GAS SUPPLY BUSINESS UNIT

Producer Marketing Ltd.

PG\&E Gas Supply Business Unit

PG\&E GAS SUPPLY BUSINESS UNIT Renaissance Energy Ltd.

PG\&E Gas Supply Business Unit

PG\&E GAS SUPPLY BUSINESS UNIT Stampeder Energy

PG\&E Gas Supply Business Unit

PG\&E GAS SUPPLY BUSINESS UNIT Talisman Energy, Inc.

PG\&E Gas Supply Business Unit

Eastport, Idaho

PG\&E GAS SUPPLY BUSINESS UNIT TransCanada Gas Services Limited PG\&E Gas Supply Business Unit

Eastport, idaho

PG\&E GAS SUPPLY BUSINESS UNIT U.S. Gas Transportation Inc.

PG\&E Gas Supply Business Unit

PG\&E GAS SUPPLY BUSINESS UNIT

Utilicorp Energy Services Ltd.

PG\&E Gas Supply Business Uni

PG\&E GAS SUPPLY BUSINESS UNIT

Valero Gas Marketing Canada inc.

PG\&E Gas Supply Business Unit

Eastport, Idaho

- N/A

283

2.03

117

1.14

400

1.77

PG\&E GAS SUPPLY BUSINESS UNIT Westcoast Gas Services Inc.

PG\&E Gas Supply Business Unit

Eastport, Idaho

$1248 \quad 1.65$

20

1.83

$1404 \quad 1.28$

$2853 \quad 1.48$

PG\&E GAS SUPPLY BUSINESS UNIT Western Gas Resources

PG\&E Gas Supply Business Unit

Eastport, Idaho

$302 \quad 1.70$

$15 \quad 2.11$

$151 \quad 1.12$

$468 \quad 1.53$

PG\&E GAS SUPPLY BUSINESS UNIT

Williams Energy Services Company

PG\&E Gas Supply Business Unit
Eastport, Idaho
Total

Avg. Price

$58 \quad 1.07$

$189 \quad 2.09$

$252 \quad 1.45$

$315 \quad 1.43$

$429 \quad 1.60$

$63 \quad 2.07$

Quarterly 
SHORT-TERM SALES

YEAR: 1997 Quarter: One

Estimated Volumes (MMCF) \& Prices (\$ / MMBTU)
Importer

Seller

Purchaser / End User

PHIBRO INC.

CoEnergy Trading Company

CNG Producing Company

PHIBRO INC.

Coastal Gas Marketing, Canada

Aquila Energy Marketing

PHIBRO INC.

Coastal Gas Marketing, Canada CNG Producing Company

PHIBRO INC

Coastal Gas Marketing, Canada

TransCanada Gas Services

PHIBRO INC.

Noram Energy Services

Aquila Energy Marketing

PHIBRO INC.

Noram Energy Services

CNG Producing Company

PHIBRO INC.

Noram Energy Services

Koch Gas Services Company

$84 \quad 4.80$

$0 \quad$ N/A

$0 \quad N / A$

84

4.80

PHIBRO INC.

SEMCO

CNG Producing Company

PHIBRO INC.

TransCanada Gas Services Limited CNG Producing Company

PHIBRO INC.

Wascana Energy Marketing Inc. CNG Producing Company

PHIBRO INC.

Wascana Energy Marketing inc.

Noram Energy Services

Point

Entry

January

Vol. Price

February

Vol. Price

March

Vol. Price

o N/A

138

3.64

Niagara Falls, NY

133

3.14

$0 \quad$ N/A

133

3.14

Niagara Falls, NY

$171 \quad 4.74$

$0 \quad N / A$

15

2.15

186

4.53

Niagara Falls, NY

$139 \quad 4.74$

$0 \quad N / A$

o N/A

139

4.74

Niagara Falls, NY

$81 \quad 4.80$

- N/A

$0 \quad$ N/A

$81 \quad 4.80$

Niagara Falls, NY

$138 \quad 3.41$

o N/A

138

Niagara Falls, NY

O N/A

o N/A

122

1.94

122

1.94

Niagara Falls, NY

$0 \quad$ N/A

$0 \quad$ N/A

$57 \quad 2.25$

57

Niagara Falls, NY

- N/A

138

3.55

o. N/A

138

3.55

Niagara Falls, NY

$84 \quad 4.97$

- N/A

o NIA

84

4.97 
Importer

Seller

Purchaser / End User

PHIBRO INC.

Wascana Energy Marketing Inc.

Piedmont Natural Gas Company

PHIBRO INC.

Wascana Energy Marketing inc.

Williams Energy Company

Niagara Falls, NY

$13 \quad 4.97$

- N/A

$0 \quad$ N/A

13

4.97

POCO MARKETING LTD.

Poco Marketing Ltd.

AIG Trading Corporation

POCO MARKETING LTD.

Poco Marketing Ltd.

AmGas Inc.

POCO MARKETING LTD.

Poco Marketing Ltd.

Cibola Corporation

POCO MARKETING LTD.

Poco Marketing Ltd.

Coastal Gas Marketing Company

Eastport, Idaho

$\begin{array}{ll}75 & 3.91\end{array}$

$17 \quad 1.18$

$0 \quad$ N/A

92

3.41

POCO MARKETING LTD.

Poco Marketing Ltd.

Coastal Gas Marketing Company

Noyes, Minnesota

$12 \quad 3.65$

$12 \quad 2.87$

$12 \quad 1.57$

36

2.71

POCO MARKETING LTD.

Poco Marketing Ltd.

Cook Inlet

Eastport, Idaho

$53 \quad 2.34$

$5 \quad 1.21$

$0 \quad$ N/A

58

2.24

POCO MARKETING LTD.

Poco Marketing Ltd.

Enserch Energy Services, Inc.

Port of Morgan, MT

$23 \quad 3.43$

$43 \quad 2.50$

$48 \quad 1.13$

114

2.11

POCO MARKETING LTD.

Poco Marketing Ltd.

IGl Resources

Eastport, Idaho

$140 \quad 1.92$

127

2.34

$141 \quad 1.51$

408

1.91

POCO MARKETING LTD.

Poco Marketing Ltd.

IGI Resources
Sumas, Washington

$603 \quad 3.61$

524

2.28

602

1.29

1729

2.39 
SHORT-TERM SALES

YEAR: 1997 Quarter: One

Estimated Volumes (MMCF) \& Prices (\$ / MMBTU)
Importer

Seller

Purchaser/End User

POCO MARKETING LTD.

Poco Marketing Ltd.

Interenergy Corporation

POCO MARKETING LTD.

Poco Marketing Ltd.

Kaztec Energy

POCO MARKETING LTD.

Poco Marketing Ltd.

Midland Cogeneration Venture

Noyes, Minnesota

- N/A

- N/A

$230 \quad 1.52$

230

1.52

POCO MARKETING LTD.

Poco Marketing Ltd.

Mock Resources

POCO MARKETING LTD.

Poco Marketing Ltd.

Mock Resources

Sumas, Washington

$6 \quad 4.11$

- N/A

- N/A

6

4.11

POCO MARKETING LTD.

Poco Marketing Ltd.

National Gas \& Electric

POCO MARKETING LTD.

Poco Marketing Ltd.

Natural Gas Clearinghouse

POCO MARKETING LTD.

Poco Marketing Ltd.

Noram Energy Services

Noyes, Minnesota

4.60

339

3.10

37

1.8

1090

3.19

POCO MARKETING LTD.

Poco Marketing Ltd

Pacific Gas \& Électric Company

Eastport, Idaho

492

1.62

69

1.08

139

1.52

POCO MARKETING LTD.

Poco Marketing Ltd.

Questar Energy Trading Co.

POCO MARKETING LTD.

Poco Marketing Ltd.

Redwood Resources Inc.
Eastport, Idaho
$64 \quad 1.39$

69

1.10

277

2.20 
SHORT-TERM SALES

YEAR: 1997 Quarter: One

Estimated Volumes (MMCF) \& Prices (\$ / MMBTU)

Importer

Seller

Purchaser / End User

POCO MARKETING LTD.

Poco Marketing Ltd.

Sacramento Municipal Utility Dist.

POCO MARKETING LTD.

Poco Marketing Ltd.

Sierra Pacific Power Company

POCO MARKETING LTD.

Poco Marketing Ltd.

Sierra Pacific Power Company

POCO MARKETING LTD.

Poco Marketing Ltd.

TransCanada Gas Services

POCO MARKETING LTD.

Poco Marketing Ltd.

Westcoast Energy inc.

Port of Morgan, MT

78

2.73

February

Vol uary

En

Eastport, Idaho

Eastport, Idaho

Sumas, Washington

Eastport, Idaho

$21 \quad 2.93$

- N/A

O N/A

21

2.93

POCO MARKETING LTD.

Poco Marketing Ltd.

Westem Gas Resources

Sumas, Washington

- N/A

o N/A

$30 \quad 1.08$

$30 \quad 1.08$

PORTLAND GENERAL ELECTRIC COMPANY

Amoco Canada Petroleum Company Ltd.

Portland General Electric Company

Eastport, Idaho

29

1.30

288

2.07

$323 \quad 1.27$

641

1.63

PORTLAND GENERAL ELECTRIC COMPANY

Crestar Energy

Portland General Electric Company

Eastport, Idaho

1.77

301

2.06

323

1.27

959

1.69

PORTLAND GENERAL ELECTRIC COMPANY

El Paso Gas Marketing Canada Inc.

Portland General Electric Company

Eastport, Idaho

$51 \quad 0.96$

62

0.72

$52 \quad 0.93$

166

0.86

PORTLAND GENERAL ELECTRIC COMPANY

Gulf Canada Resources Limited

Portland General Electric Company

Eastport, Idaho

PORTLAND GENERAL ELECTRIC COMPANY

IGI Resources

Portland General Electric Company

Eastport, Idaho
$95 \quad 1.00$

86

0.99

9

0.88

277

0.99 
SHORT-TERM SALES

YEAR: 1997 Quarter: One

Estimated Volumes (MMCF) \& Prices (\$ / MMBTU)

Importer

Seller

Purchaser/End User

PORTLAND GENERAL ELECTRIC COMPANY

Renaissance Energy Ltd.

Portland General Electric Company

Eastport, Idaho

PORTLAND GENERAL ELECTRIC COMPANY

Valero Gas Marketing Canada Inc.

Portiand General Electric Company

Eastport, Idaho

Port of Morgan, MT

Eastport, Idaho

PROGAS U.S.A., INC

ProGas Limited

ERI Services

Point

Entry

PROGAS U.S.A., INC.

ProGas Limited

Cibola Corporation

PROGAS U.S.A., INC.

ProGas Limited

Ensource

Eastport, Idaho

Noyes, Minnesota

ProGas Limited

Kimball Trading Company, L.L.C.

Niagara Falls, NY

ProGas Limited

Koch Gas Services Company

PROGAS U.S.A., INC.

ProGas Limited

Michigan Consolidated Gas Company

Noyes, Minnesota

$306 \quad 3.07$

331

2.71

$373 \quad 1.59$

1010

2.41

PROGAS U.S.A., INC.

ProGas Limited

Natural Gas Clearinghouse

Eastport, Idaho

- N/A

$0 \quad N / A$

73

1.35

73

1.35

PROGAS U.S.A., INC.

ProGas Limited

Petro Source Gas Ventures

Waddington, NY

$180 \quad 5.03$

162

4.00

180

2.79

522

3.94

PROGAS U.S.A., INC

ProGas Limited

StampGas

Port of Morgan, MT
215

1.53

622

2.12 $\begin{array}{llll}215 & 2.80 & 192 & 2.02\end{array}$ 


\section{SHORT-TERM SALES \\ YEAR: 1997 Quarter: One}

Estimated Volumes (MMCF) \& Prices ( $/$ MMBTU)

Importer

Seller

Purchaser/End User

PROGAS U.S.A., INC.

ProGas Limited

Tenaska Marketing Ventures

PROGAS U.S.A., INC.

ProGas Limited

Tenaska Marketing Ventures

PUGET SOUND ENERGY

Talisman LT

Puget Sound Energy

QUESTAR ENERGY TRADING COMPANY

\section{B.C. Gas Inc.}

Questar Energy Trading Co.

RENAISSANCE ENERGY (U.S.), INC.

Renaissance Energy Ltd.

Renaissance Energy (U.S.) Inc.

Niagara Falls, NY

$91 \quad 3.32$

$91 \quad 3.32$

$90 \quad 2.32$

272

2.99

RENAISSANCE ENERGY (U.S.), INC.

Renaissance Energy Ltd.

Renaissance Energy (U.S.) Inc.

Noyes, Minnesota

814

2.94

81

2.94

735

1.79

2362

2.58

RENAISSANCE ENERGY (U.S.), INC.

Renaissance Energy Ltd.

Renaissance Energy (U.S.) Inc.

Port of Morgan, MT

1625

3.11

1518

2.35

168

1.24

4824

2.22

RENAISSANCE ENERGY (U.S.), INC.

Renaissance Energy Ltd.

Renaissance Energy (U.S.) Inc.

Waddington, NY

699

3.13

699

3.13

367

2.23

1764

2.94

ROCHESTER GAS \& ELECTRIC CORPORATION

Coastal Gas Marketing, Canada

Rochester Gas \& Electric Company

Grand Island, NY

30

281

3.61

$321 \quad 2.88$

905

3.81

ROCHESTER GAS \& ELECTRIC CORPORATION

Westcoast Gas Services Inc.

Rochester Gas \& Electric Company

Grand Island, NY

281

3.60

320

2.87

905

3.80

ROCHESTER GAS \& ELECTRIC CORPORATION

Western Gas Resources

Rochester Gas \& Electric Company

Grand Island, NY
4.98

281

3.61

321

2.88

905

3.81 
Importe

Seller

Purchaser/End User

SALMON RESOURCES LTD

Shell Canada Limited

City of Ellensburg, WA

SALMON RESOURCES LTD.

Shell Canada Limited

Sierra Pacific Power Company

SALMON RESOURCES LTD.

Shell Canada Limited

Various CA State Markets

SAN DIEGO GAS \& ELECTRIC

AIG Trading Corporation

San Diego Gas \& Electric Company

SAN DIEGO GAS \& ELECTRIC

Amoco Canada Petroleum Company Ltd.

San Diego Gas \& Electric Company

SAN DIEGO GAS \& ELECTRIC

B.C. Gas inc.

San Diego Gas \& Electric Company

SAN DIEGO GAS \& ELECTRIC

Imperial Oil Resources

San Diego Gas \& Electric Company

SAN DIEGO GAS \& ELECTRIC

TransCanada Gas Services Limited

San Diego Gas \& Electric Company

SELKIRK COGEN PARTNERS, L.P.

AIG Trading Corporation

Selkirk Cogeneration Partners

SELKIRK COGEN PARTNERS, L.P.

CoEnergy Trading Company

Selkirk Cogeneration Partners

SELKIRK COGEN PARTNERS, L.P.

Howard Energy Company, inc.

Selkirk Cogeneration Partners
Niagara Falls, NY

Sumas, Washington

Sumas, Washington

Eastport, Idaho

Eastport, Idaho

Eastport, Idaho

Eastport, Idaho

Eastport, Idaho

Niagara Falls, NY

1.83
Niagara Falls, NY
$305 \quad 5.03$

$11 \quad 2.18$

O N/A

316

4.93

$0 \quad$ N/A

- N/A

2.10

31

2.10

Quarterly

Total Avg.

133

2.03

401

52

1.19

82

2.28

$486 \quad 1$

$342 \quad 1.55$

359

1187

1.25

$0 \quad$ N/A

$0 \quad$ N/A

30

3.07
SHORT-TERM SALES

YEAR: 1997 Quarter: One

Estimated Volumes (MMCF) \& Prices (\$ / MMBTU) 


\section{SHORT-TERM SALES \\ YEAR: 1997 Quarter: One}

Estimated Volumes (MMCF) \& Prices (\$ / MMBTU)

Importer

Seller

Purchaser / End User

SELKIRK COGEN PARTNERS, L.P.

Ronaissance Energy Ltd.

Selkirk Cogeneration Partners

SELKIRK COGEN PARTNERS, L.P.

Renaissance Energy Ltd.

Selkirk Cogeneration Partners

Point

of

Entry

January

Vol. Price

February

Vol. Price

March

Vol. Price

Quarterly

Total

Vol. Prg. Price

Niagara Falls, NY

$28 \quad 4.97$

$0 \quad$ N/A

$0 \quad$ N/A

28

4.97

Waddington, NY

$18 \quad 2.96$

O N/A

O N/A

18

2.96

SELKIRK COGEN PARTNERS, L.P.

Wascana Energy Marketing Inc.

Selkirk Cogeneration Partners

Waddington, NY

$0 \quad$ N/A

O N/A

$11 \quad 2.47$

11

2.47

SEMCO ENERGY SERVICES

CC Pace

NUI Corporation

Niagara Falls, NY

- N/A

$0 \quad$ N/A

$4 \quad 1.95$

$4 \quad 1.95$

SEMCO ENERGY SERVICES

Consumers' Gas Company Limited Noram Energy Services

Niagara Falls, NY

$6 \quad 4.76$

O N/A

O N/A

$6 \quad 4.76$

SEMCO ENERGY SERVICES

El Paso Gas Marketing Canada Inc.

NUI Corporation

Niagara Falls, NY

- N/A

$0 \quad$ N/A

$\begin{array}{ll}76 & 2.01\end{array}$

76

2.01

SEMCO ENERGY SERVICES

Midoon Gas Services Corporation

National Fuel Resources

Niagara Falls, NY

$5 \quad 3.90$

$0 \quad$ N/A

$0 \quad$ N/A

$5 \quad 3.90$

SEMCO ENERGY SERVICES

TransCanada Gas Services Limited

National Fuel Gas Distribution

Niagara Falls, NY

$0 \quad$ N/A

$0 \quad$ N/A

$16 \quad 2.09$

$16 \quad 2.09$

SEMCO ENERGY SERVICES

Wascana Energy Marketing Inc.

National Fuel Gas Distribution

Niagara Falls, NY

o. N/A

$14 \quad 2.09$

$0 \quad N / A$

14

2.09

SEMCO ENERGY SERVICES

Wascana Energy Marketing inc.

Noram Energy Services

Niagara Falls, NY

$20 \quad 4.70$

0

N/A

$0 \quad N / A$

20

4.70

SEMCO ENERGY SERVICES

Wascana Energy Marketing Inc.

Williams Energy Company
Niagara Falls, NY
$5 \quad 2.58$

N/A

5

2.58 


\section{SHORT-TERM SALES \\ YEAR: 1997 Quarter: One \\ Estimated Volumes (MMCF) \& Prices (\$ / MMBTU)}

Importer

Seller

Purchaser / End User

SIERRA PACIFIC POWER COMPANY Amoco Canada Marketing Corporation Sierra Pacific Power Company

SIERRA PACIFIC POWER COMPANY Chevron Canada Resources

Sierra Pacific Power Company

SIERRA PACIFIC POWER COMPANY IGI Resources

Sierra Pacific Power Company

SIERRA PACIFIC POWER COMPANY PanEnergy Gas Services, inc.

Sierra Pacific Power Company

SIERRA PACIFIC POWER COMPANY

Salmon Resources Ltd.

Sierra Pacific Power Company

SIERRA PACIFIC POWER COMPANY

Westcoast Gas Services Inc.

Sierra Pacific Power Company

SONAT MARKETING COMPANY

EC\&T Resources Canada

Buffalo Paperboard Corporation

SONAT MARKETING COMPANY

EC\&T Resources Canada

Commonwealth Energy Services

SONAT MARKETING COMPANY

Selkirk Cogeneration Partners, L.P.

CNG Energy Services

Waddington, NY

$0 \quad$ N/A

O N/A

$5 \quad 2.42$

$5 \quad 2.42$

SONAT MARKETING COMPANY Selkirk Cogeneration Partners, L.P. New England Power Company

January
Vol. Pric

$612 \quad 1.32$

$307 \quad 1.12$

$277 \quad 1.13$

307

1.12

890

1.12

Eastport, Idaho

$153 \quad 1.32$

$138 \quad 1.32$

154

1.32

446

1.32

Eastport, Idaho

$458 \quad 1.34$

$414 \quad 1.34$

459

1332

1.34

Sumas, Washington

$153 \quad 1.71$

$124 \quad 1.71$

138

416

1.71

Eastport, Idaho

$307 \quad 1.11$

277

1.12

30

1.10

890

1.11

Grand Island, NY

O N/A

$55 \quad 3.53$

$0 \quad N / A$

55

3.53

Grand Island, NY

$0 \quad$ N/A

$12 \quad 3.53$

- N/A

12

3.53

Wadidington, NY

- N/A

- N/A

15

2.42

15

2.42

SOUTHERN CALIFORNIA EDISON COMPANY

Amoco Canada Petroleum Company Ltd.

Southem Califomia Edison Company

Eastport, Idaho
$0 \quad$ N/A

0

N/A

190

1.29

190

1.29 


\section{SHORT-TERM SALES \\ YEAR: 1997 Quarter: One \\ Estimated Volumes (MMCF) \& Prices ( $\$$ / MMBTU)}

Importer

Seller

Purchaser/End User

Point

of

Entry
January

Vol. Price
Eastport, Idaho

CRN CALIFORNIA EDISON COMPANY

Aquila Canada Corporation

Southem Califomia Edison Company

SOUTHERN CALIFORNIA EDISON COMPANY

Cook Inlet Energy Supply

Southem Califomia Edison Company

Eastport; Idaho

SOUTHERN CALIFORNIA EDISON COMPANY

Direct Canada

Southem California Edison Company

Eastport, Idaho

$96 \quad 2.59$

$173 \quad 2.04$

$10 \quad 1.20$

279

2.20

SOUTHERN CALIFORNIA EDISON COMPANY

Equitable Resources Marketing Canad

Southem Califomia Edison Company

Eastport, Idaho

$0 \quad$ N/A

$135 \quad 2.27$

Eastport, Idaho

SOUTHERN CALIFORNIA EDISON COMPANY

Pan-Alberta Gas Ltd.

Southem Califomia Edison Company

SOUTHERN CALIFORNIA EDISON COMPANY

TransCanada Gas Services Inc.

Southem California Edison Company

Eastport, Idaho

125

2.08

- N/A

0 N/A

125

2.08

ST. CLAIR PIPELINES LTD.

St. Clair Pipelines Ltd.

Williams Energy Company

Niagara Falls, NY

O N/A

$10 \quad 2.99$

$226 \quad 2.00$

235

2.04

ST. LAWRENCE GAS COMPANY, INC.

AEC Oil \& Gas Company

Various NY State Markets

Massena, New York

O N/A

$0 \quad$ N/A

$33 \quad 1.92$

33

1.92

ST. LAWRENCE GAS COMPANY, INC.

Cibola Canada Energy Marketing Co.

Various NY State Markets

Massena, New York

$33 \quad 2.65$

O N/A

O N/A

33

2.65

ST. LAWRENCE GAS COMPANY, INC Enron Capital \& Trade Resources Can Various NY State Markets

Massena, New York

- N/A

$30 \quad 3.09$

- N/A

30

3.09

ST. LAWRENCE GAS COMPANY, INC PanEnergy Marketing Canada

Various NY State Markets
Massena, New York
$140 \quad 3.09$

104

2.84

399

3.16 


\section{SHORT-TERM SALES \\ YEAR: 1997 Quarter: One \\ Estimated Volumes (MMCF) \& Prices (\$ / MMBTU)}

Importer

Seller

Purchaser / End User

STAMPGAS (U.S.) INC.

Stampeder Energy

Alliance Energy Services

STAMPGAS (U.S.) INC.

Stampeder Energy

Utilicorp United

SUMAS COGENERATION COMPANY, LP (SP)

Westcoast Gas Services inc.

Sumas Cogeneration

Sumas, Washington

SUNCOR INC.

Suncor inc.

AIG Trading Corporation

Eastport, Idaho

Port of Morgan, MT

Eastport, Idaho

Port of Morgan, MT

Suncor Inc.

Inland Steel Company

SUNCOR INC.

Suncor inc.

Minnegasco

SUNCOR INC.

Suncor Inc.

Noram Energy Services

Eastport, Idaho

Port of Morgan, MT

SUNCOR INC

Suncor inc.

TransCanada Gas Services
Eastport, Idaho
$23 \quad 2.11$

10

2.50

$11 \quad 2.50$

44

2.29

$155 \quad 3.91$

140

2.45

155

1.33

450

2.57

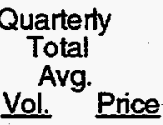

$10 \quad 1.55$

$900 \quad 1.59$

$641 \quad 2.53$

$520 \quad 2.59$

$900 \quad 2.57$

$900 \quad 1.59$

$\begin{array}{llllllll}248 & 2.32 & 234 & 1.43 & 259 & 0.99 & 741 & 1.57\end{array}$

SP Intermuptible/special purchase sale made under Long-term authorization. 


\section{SHORT-TERM SALES \\ YEAR: 1997 Quarter: One \\ Estimated Volumes (MMCF) \& Prices (\$ / MMBTU)}

Importer

Seller

Purchaser / End User

SUNCOR INC.

Suncor Inc.

Vaiero Gas Marketing

SUNCOR INC.

Suncor inc.

Wisconsin Power \& Light

TENASKA MARKETING VENTURES

Coastal Gas Marketing, Canada

Various MN State Markets

TENASKA MARKETING VENTURES

Tenaska Marketing Canada

Various IA State Markets

TENASKA MARKETING VENTURES

Tenaska Marketing Canada

Various MN State Markets

TENASKA MARKETING VENTURES

Wascana Energy Marketing Inc.

Various MN State Markets

Noyes, Minnesota

Hidalgo, Texas

PEMEX

Texaco Natural Gas

THE BROOKLYN UNION GAS COMPANY AIG Trading Corporation

Brooklyn Union Gas

Niagara Falis, NY

- N/A

10

2.15

$0 \quad$ N/A

10

2.15

THE BROOKLYN UNION GAS COMPANY Natural Gas Clearinghouse

Brooklyn Union Gas

Niagara Falls, NY

- N/A

$11 \quad 2.19$

- N/A

11

2.19

THE BROOKLYN UNION GAS COMPANY Renaissance Energy Ltd.

Brooklyn Union Gas

Niagara Falls, NY

- N/A

10

2.10

$0 \quad$ N/A

10

2.10

THE BROOKLYN UNION GAS COMPANY TransCanada Gas Services Limited Brooklyn Union Gas
Niagara Falls, NY
20

2.12
Quarterly

Tota

Vol. ${ }^{\text {Avg. }}$ Price

$333 \quad 2.55$

$003 \quad 2.57$

$12 \quad 2.00$

2.21
$4 \quad 1.90$ 
SHORT-TERM SALES

YEAR: 1997 Quarter: One

Estimated Volumes (MMCF) \& Prices (\$ / MMBTU)
Importer

Seller

Purchaser / End User

THE MONTANA POWER COMPANY

TransCanada Gas Services Inc.

Montana Power

TRANSCANADA GAS SERVICES INC.

CanStates Gas Marketing

TransCanada Gas Services

TRANSCANADA GAS SERVICES INC. TransCanada Gas Services Limited ADP

Point
of

Babb, Montana

Eastport, Idaho

Port of Morgan, MT

Eastport, Idaho

TRANSCANADA GAS SERVICES INC. TransCanada Gas Services Limited AlG Trading Corporation

TRANSCANADA GAS SERVICES INC. TransCanada Gas Services Limited AlG Trading Corporation

TRANSCANADA GAS SERVICES INC. TransCanada Gas Services Limited

Amerada Hess

Port of Morgan, MT

$13 \quad 3.75$

$11 \quad 2.41$

$15 \quad 1.21$

39

2.38

TRANSCANADA GAS SERVICES INC TransCanada Gas Services Limited Amoco Trading

Eastport, Idaho

Eastport, Idaho

TRANSCANADA GAS SERVICES INC.

TransCanada Gas Services Limited

Aquila Energy Marketing

TRANSCANADA GAS SERVICES INC.

TransCanada Gas Services Limited

Aquila Energy Marketing

Port of Morgan, MT

O N/A

O N/A

$79 \quad 1.21$

79

TRANSCANADA GAS SERVICES INC. TransCanada Gas Services Limited

Asarco Inc.

Babb, Montana

$0 \quad$ N/A

$25 \quad 1.18$

$41 \quad 1.17$

65

1.17

TRANSCANADA GAS SERVICES INC

TransCanada Gas Services Limited

Austin 


\section{SHORT-TERM SALES \\ YEAR: 1997 Quarter: One \\ Estimated Volumes (MMCF) \& Prices (\$ / MMBTU)}

Importer

Seller

Purchaser/End User

TRANSCANADA GAS SERVICES INC. TransCanada Gas Services Limited Barret Fuels

TRANSCANADA GAS SERVICES INC. TransCanada Gas Services Limited Barretts Minerals

TRANSCANADA GAS SERVICES INC. TransCanada Gas Services Limited Cibola Corporation

TRANSCANADA GAS SERVICES INC. TransCanada Gas Services Limited CoEnergy Trading Company

TRANSCANADA GAS SERVICES INC. TransCanada Gas Services Limited CoEnergy Trading Company

TRANSCANADA GAS SERVICES INC. TransCanada Gas Services Limited Coastal Gas Marketing Company

TRANSCANADA GAS SERVICES INC. TransCanada Gas Services Limited

Coastal Gas Marketing Company

TRANSCANADA GAS SERVICES INC TransCanada Gas Services Limited Coastal Gas Marketing Company

TRANSCANADA GAS SERVICES INC. TransCanada Gas Services Limited Coastal Gas Marketing Company

TRANSCANADA GAS SERVICES INC. TransCanada Gas Services Limited ConAgra Energy

Port of Morgan, MT

$111 \quad 3.75$

$101 \quad 2.41$

o N/A

212

3.11

TRANSCANADA GAS SERVICES INC

TransCanada Gas Services Limited

Consumers Power Company

Point

Entry

anuary

Vol. Price

Vol. Price

March

Vol. Price

Quarterly

Total

Vol. Price

Sumas, Washington

$1405 \quad 3.75$

O N/A

o N/A

1405

3.75

Waddington, NY

$50 \quad 4.27$

o N/A

o N/A

50

4.27

Eastport, Idaho

Noyes, Minnesota

347

3.75

$3130 \quad 2.67$

$3483 \quad 1.65$

10089

2.69

Port of Morgan, MT

o N/A

153

2.41

$155 \quad 1.21$

308

1.81

Sumas, Washington

162

3.97

$0 \quad N / A$

$65 \quad 1.25$

227

3.19

Noyes, Minnesota

$1395 \quad 3.75$

121

2.67

$1479 \quad 1.65$

4092

2.67 


\section{SHORT-TERM SALES \\ YEAR: 1997 Quarter: One \\ Estimated Volumes (MMCF) \& Prices (\$ / MMBTU)}

Importer

Seller

Purchaser / End User

TRANSCANADA GAS SERVICES INC. TransCanada Gas Services Limited Cook Inlet

TRANSCANADA GAS SERVICES INC. TransCanada Gas Services Limited Cook Inlet

TRANSCANADA GAS SERVICES INC. TransCanada Gas Services Limited ERI Services

TRANSCANADA GAS SERVICES INC. TransCanada Gas Services Limited Eastem Energy Marketing

TRANSCANADA GAS SERVICES INC. TransCanada Gas Services Limited Enron Capital \& Trade Resources

TRANSCANADA GAS SERVICES INC. TransCanada Gas Services Limited Enron Capital \& Trade Resources

TRANSCANADA GAS SERVICES INC. TransCanada Gas Services Limited Enron Capital \& Trade Resources

TRANSCANADA GAS SERVICES INC. TransCanada Gas Services Limited Enserch Energy Services, Inc.

Eastport, Idaho

$18 \quad 3.38$

O N/A

36

1.55

TRANSCANADA GAS SERVICES INC. TransCanada Gas Services Limited Enserco Energy, Inc.

Eastport, Idaho

$0 \quad$ N/A

O N/A

$1 \quad 1.55$

TRANSCANADA GAS SERVICES INC. TransCanada Gas Services Limited Ensource

Eastport, idaho

- N/A

157

2.11

$0 \quad$ N/A

157

TRANSCANADA GAS SERVICES INC. TransCanada Gas Services Limited Fuel Imbalance
$26 \quad 3.38$

31

2.11

40

1.55

96

2.22
54

$1 \quad 1.55$

Quarterly
Total
Avg. Price
Vol. Price

254

2.48

4.10 3.97 


\section{SHORT-TERM SALES \\ YEAR: 1997 Quarter: One \\ Estimated Volumes (MMCF) \& Prices (\$ / MMBTU)}

Importer

Seller

Purchaser / End User

TRANSCANADA GAS SERVICES INC TransCanada Gas Services Limited Fuel imbalance

TRANSCANADA GAS SERVICES INC. TransCanada Gas Services Limited Fuel imbalance

TRANSCANADA GAS SERVICES INC. TransCanada Gas Services Limited Holnam Inc.

Babb, Montana

Noyes, Minnesota

Port of Morgan, MT

TransCanada Gas Services Limited

Hutchinson

Point

of

Noyes, Minnesota

Waddington, NY

$8 \quad 4.27$

$\begin{array}{ll}7 & 3.34\end{array}$

$8 \quad 2.75$

22

3.46

TRANSCANADA GAS SERVICES INC TransCanada Gas Services Limited Howand Energy Company

TRANSCANADA GAS SERVICES INC. TransCanada Gas Services Limited

IGI Resources

Eastport, Idaho

Babb, Montana

TransCanada Gas Services Limited

Interenergy Corporation

TRANSCANADA GAS SERVICES INC. TransCanada Gas Services Limited Interenergy Corporation

Sumas, Washington

- N/A

$0 \quad$ N/A

$3 \quad 1.25$

3

1.25

TRANSCANADA GAS SERVICES INC. TransCanada Gas Services Limited

J. Aaron Company

Eastport, ldaho

O N/A

196

2.11

$0 \quad$ N/A

196

2.11

TRANSCANADA GAS SERVICES INC. TransCanada Gas Services Limited Kaztex Energy Management

Port of Morgan, MT

$0 \quad N / A$

0

N/A

126

1.21

126

1.21

TRANSCANADA GAS SERVICES INC. TransCanada Gas Services Limited Koch Gas Services Company
Port of Morgan, MT

310

3.75

0

N/A

309

1.21

619

2.48 


\section{SHORT-TERM SALES \\ YEAR: 1997 Quarter: One \\ Estimated Volumes (MMCF) \& Prices (\$ / MMBTU)}

Importer

Seller

Purchaser/End User

TRANSCANADA GAS SERVICES INC.

TransCanada Gas Services Limited

Long Island Lighting Company

TRANSCANADA GAS SERVICES INC.

TransCanada Gas Services Limited

Louisiana Pacific

TRANSCANADA GAS SERVICES INC.

TransCanada Gas Services Limited

Michigan Consolidated Gas Company

TRANSCANADA GAS SERVICES INC.

TransCanada Gas Services Limited

MidAmerican Energy Company

TRANSCANADA GAS SERVICES INC.

TransCanada Gas Services Limited

Minnegasco

TRANSCANADA GAS SERVICES INC.

TransCanada Gas Senvices Limited

Mock Resources

Eastport, Idaho

$15 \quad 3.38$

- N/A

$0 \quad$ N/A

15

3.38

TRANSCANADA GAS SERVICES INC.

TransCanada Gas Services Limited

Mock Resources

Sumas, Washington

- N/A

$\begin{array}{ll}63 & 2.28\end{array}$

$133 \quad 1.25$

196

1.58

TRANSCANADA GAS SERVICES INC.

TransCanada Gas Services Limited

Montana Power

Babb, Montana

$609 \quad 1.32$

550

1.18

609

1.17

1769

1.22

TRANSCANADA GAS SERVICES INC.

TransCanada Gas Services Limited

Montana Resources

Babb, Montana

$35 \quad 1.32$

22

1.18

23

1.17

80

1.24

TRANSCANADA GAS SERVICES INC. TransCanada Gas Services Limited

NPGA

Port of Morgan, MT

- N/A

0

N/A

$27 \quad 1.21$

27

TRANSCANADA GAS SERVICES INC. TransCanada Gas Services Limited NSP 


\section{SHORT-TERM SALES \\ YEAR: 1997 Quarter: One \\ Estimated Volumes (MMCF) \& Prices (\$ / MMBTU)}

Importer
Seller

Purchaser / End User

TRANSCANADA GAS SERVICES INC

TransCanada Gas Services Limited

National Gas \& Electric

Point

of

Entry

Noyes, Minnesota

$22 \quad 3.94$

- N/A

- N/A

22

3.94

TRANSCANADA GAS SERVICES INC. TransCanada Gas Services Limited National Gas \& Electric

Port of Morgan, MT

139

3.75

1260

2.41

411

1.21

3066

2.86

TRANSCANADA GAS SERVICES INC. TransCanada Gas Services Limited Natural Gas Clearinghouse

Eastport, Idaho

Eastport, Idaho

TransCanada Gas Services Limited Noram Energy Services

TRANSCANADA GAS SERVICES INC. TransCanada Gas Services Limited Noram Energy Services

Port of Morgan, MT

$0 \quad$ N/A

140

2.41

- N/A

140

2.41

TRANSCANADA GAS SERVICES INC. TransCanada Gas Services Limited Northem States Power Company

Noyes, Minnesota

489

3.75

414

2.67

O N/A

903

3.26

TRANSCANADA GAS SERVICES INC. TransCanada Gas Services Limited Northshore Gas Company

Noyes, Minnesota

1108

3.75

998

2.67

111

1.65

3217

2.69

TRANSCANADA GAS SERVICES INC. TransCanada Gas Services Limited Northwest Natural Gas Company

Eastport, idaho

TRANSCANADA GAS SERVICES INC. TransCanada Gas Services Limited Northwest Natural Gas Company

TRANSCANADA GAS SERVICES INC. TransCanada Gas Services Limited Northwest Public Service

Port of Morgan, MT

29

3.75

$214 \quad 2.41$

$62 \quad 1.21$

576

2.98

TRANSCANADA GAS SERVICES INC. TransCanada Gas Services Limited Pacific Gas \& Electric Company
Eastport, Idaho
$284 \quad 3.38$

$357 \quad 2.11$

63

1.55

704

2.57 


\section{SHORT-TERM SALES \\ YEAR: 1997 Quarter: One \\ Estimated Volumes (MMCF) \& Prices (\$ / MMBTU)}

Importer

Seller

Purchaser/End User

TRANSCANADA GAS SERVICES INC.

TransCanada Gas Services Limited

PanEnergy Trading \& Marketing Svcs.

TRANSCANADA GAS SERVICES INC. TransCanada Gas Services Limited

PanEnergy Trading \& Marketing Svcs.

TRANSCANADA GAS SERVICES INC.

TransCanada Gas Services Limited

PanEnergy Trading \& Marketing Svcs.

TRANSCANADA GAS SERVICES INC.

TransCanada Gas Services Limited

Peoples Gas, Light, \& Coke

TRANSCANADA GAS SERVICES INC. TransCanada Gas Services Limited

Phibro Oil \& Gas Inc.

Waddington, NY

$111 \quad 4.27$

O N/A

O N/A

111

4.27

TRANSCANADA GAS SERVICES INC. TransCanada Gas Services Limited Quick Trade

Port of Morgan, MT

o N/A

112

2.41

- N/A

112

2.41

TRANSCANADA GAS SERVICES INC. TransCanada Gas Services Limited Rhone-Poulenc

Babb, Montana

o. N/A

O N/A

28

1.17

28

1.17

TRANSCANADA GAS SERVICES INC. TransCanada Gas Services Limited San Diego Gas \& Electric Company

TRANSCANADA GAS SERVICES INC. TransCanada Gas Services Limited Sierra Pacific Power Company

Eastport, Idaho

- N/A

- N/A

$7 \quad 1.55$

$7 \quad 1.55$

TRANSCANADA GAS SERVICES INC. TransCanada Gas Services Limited Sierra Pacific Power Company

Sumas, Washington

$3 . \quad 3.97$

- N/A

o N/A

$3 \quad 3.97$

TRANSCANADA GAS SERVICES INC. TransCanada Gas Services Limited Southwest Gas Compration
Eastport, Idaho
O N/A

O N/A

29 


\section{SHORT-TERM SALES \\ YEAR: 1997 Quarter: One \\ Estimated Volumes (MMCF) \& Prices (\$ / MMBTU)}

Importer

Seller

Purchaser / End User

TRANSCANADA GAS SERVICES INC.

TransCanada Gas Services Limited

Southwest Gas Corporation

TRANSCANADA GAS SERVICES INC

TransCanada Gas Services Limited

Stone Container Corporation

TRANSCANADA GAS SERVICES INC.

TransCanada Gas Services Limited

Superior

Point

of

Entry

Sumas, Washington

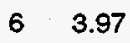

o N/A

- N/A

6

3.97

Babb, Montana

$289 \quad 1.32$

o N/A

107

1.17

396

1.28

Port of Morgan, MT

- N/A

$0 \quad$ N/A

14

1.2

142

1.21

TRANSCANADA GAS SERVICES INC.

TransCanada Gas Services Limited

Tenaska Marketing Ventures

Port of Morgan, MT

$0 \quad$ N/A

$0 \quad$ N/A

$310 \quad 1.21$

310

1.21

TRANSCANADA GAS SERVICES INC.

TransCanada Gas Services Limited

Tenaska Marketing Ventures

Sumas, Washington

0. N/A

$0 \quad$ N/A

$\begin{array}{ll}73 & 1.25\end{array}$

73

1.25

TRANSCANADA GAS SERVICES INC. TransCanada Gas Services Limited

Terra International

Port of Morgan, MT

484

3.75

429

2.41

422

1.21

1334

2.52

TRANSCANADA GAS SERVICES INC. TransCanada Gas Services Limited

Texaco Natural Gas

Niagara Falls, NY

$15 \quad 4.10$

$0 \quad$ N/A

$0 \quad$ N/A

15

4.10

TRANSCANADA GAS SERVICES INC. TransCanada Gas Services Limited U.S. Gas Transportation inc.

Eastport, Idaho

$13 \quad 3.38$

45

2.11

517

1.55

575

1.64

TRANSCANADA GAS SERVICES INC. TransCanada Gas Services Limited

U.S. Gas Transportation Inc.

Sumas, Washington

$\begin{array}{ll}63 & 3.97\end{array}$

247

2.28

- N/A

310

2.62

TRANSCANADA GAS SERVICES INC. TransCanada Gas Services Limited

Universal Resources

Eastport, Idaho

- N/A

$7 \quad 2.11$

O N/A

7

2.11

TRANSCANADA GAS SERVICES INC. TransCanada Gas Services Limited Universal Resources
Sumas, Washington
59

2.28
0

N/A

209

3.49 
SHORT-TERM SALES

YEAR: 1997 Quarter: One

Estimated Volumes (MMCF) \& Prices (\$ / MMBTU) importer

Seller

Purchaser/End User

TRANSCANADA GAS SERVICES INC TransCanada Gas Services Limited Utilicorp United

TRANSCANADA GAS SERVICES INC. TransCanada Gas Services Limited Wascana Energy Marketing

TRANSCANADA GAS SERVICES INC. TransCanada Gas Services Limited Washington Water Power Company

TRANSCANADA GAS SERVICES INC. TransCanada Gas Services Limited Westcoast Gas Services (U.S.A.) Inc

TRANSCANADA GAS SERVICES INC. TransCanada Gas Services Limited Westcoast Gas Services Inc.

Noyes, Minnesota

261

3.75

- N/A

- N/A

261

3.75

TRANSCANADA GAS SERVICES INC. TransCanada Gas Services Limited Western Gas Resources

Eastport, Idaho

15

3.38

$0 \quad$ N/A

- N/A

154

TRANSCANADA GAS SERVICES INC. TransCanada Gas Services Limited Western Gas Resources

Niagara Falls, NY

4.10

$0 \quad N / A$

o. N/A

10

4.10

TRANSCANADA GAS SERVICES INC. TransCanada Gas Services Limited Western Gas Resources

Sumas, Washington

$5 \quad 3.97$

O N/A

$0 \quad$ N/A

$5 \quad 3.97$

TRANSCANADA GAS SERVICES INC TransCanada Gas Services Limited Wickford Energy Marketing Inc.

Eastport, Idaho

$0 \quad$ N/A

32

2.11

$0 \quad$ N/A

32

2.11

TRANSCANADA GAS SERVICES INC. TransCanada Gas Services Limited Wickford Energy Marketing Inc.

Niagara Falls, NY

$64 \quad 4.10$

$7 \quad 3.29$

$13 \quad 2.20$

84

TRANSCANADA GAS SERVICES INC TransCanada Gas Services Limited Wickford Energy Marketing Inc.
Waddington, NY o N/A

N/A 
SHORT-TERM SALES

YEAR: 1997 Quarter: One

Estimated Volumes (MMCF) \& Prices (\$/MMBTU)
Importer

Seller

Purchaser / End User

TRANSCANADA GAS SERVICES INC.

TransCanada Gas Services Limited

Williams Energy Company

TRANSCANADA GAS SERVICES INC.

TransCanada Gas Services Limited

Wisconsin Electric

Point

Entry

January
Vol. Price

Port of Morgan, MT

- N/A

$84 \quad 2.41$

$62 \quad 1.21$

146

1.90

Port of Morgan, MT

$\begin{array}{ll}75 & 3.75\end{array}$

$\begin{array}{ll}68 & 2.41\end{array}$

$75 \quad 1.21$

217

2.46

TRANSCANADA GAS SERVICES INC.

TransCanada Gas Services Limited

Wisconsin Gas

Noyes, Minnesota

$31 \quad 3.75$

$28 \quad 2.67$

$31 \quad 1.65$

91

2.69

TRANSCANADA GAS SERVICES INC.

TransCanada Gas Services Limited

Wisconsin Power \& Light

Noyes, Minnesota

$310 \quad 3.75$

- N/A

o N/A

310

3.75

TRANSCANADA GAS SERVICES INC.

TransCanada Gas Services Limited

Wisconsin Public Service Corp.

Port of Morgan, MT

- N/A

- N/A

$13 \quad 1.21$

13

1.21

TRANSCO ENERGY MARKETING COMPANY

CanStates Gas Marketing

CoEnergy Trading Company

Niagara Falls, NY

O N/A

19

2.14

0

N/A

19

2.14

TRANSCO ENERGY MARKETING COMPANY

CanStates Gas Marketing

National Fuel Gas Distribution

Niagara Falls, NY

O N/A

$3 \quad 2.07$

- N/A

3

2.07

TRANSCO ENERGY MARKETING COMPANY CanStates Gas Marketing

National Fuel Resources

Niagara Falls, NY

$0 \quad$ N/A

23.08

- N/A

2

3.08

TRANSCO ENERGY MARKETING COMPANY

CanStates Gas Marketing

TransCanada Gas Services

Niagara Falls, NY

O N/A

19

3.17

O N/A

19

3.17

TRANSCO ENERGY MARKETING COMPANY

CanStates Gas Marketing

Wascana Energy Marketing

Niagara Falls, NY

$14 \quad 2.06$

o N/A

17

2.23

TRANSCO ENERGY MARKETING COMPANY

Cowest Energy

Continental Natural Gas

Niagara Falls, NY

$2.3 .35 \quad 14 \quad 2.06$

o N/A

O N/A

34

2.32

34

2.32 
SHORT-TERM SALES

YEAR: 1997 Quarter: One

Estimated Volumes (MMCF) \& Prices (\$/MMBTU)
Importer

Seller

Purchaser / End User

TRANSCO ENERGY MARKETING COMPANY

Cowest Energy

TransCanada Gas Services

Niagara Falis, NY

TRANSCO ENERGY MARKETING COMPANY

Cowest Energy

Wascana Energy Marketing

Niagara Falls, NY

UNION PACIFIC FUELS, INC.

PEMEX

Union Pacific Fuels

Hidalgo, Texas

Hidalgo, Texas

PEMEX

Valero Gas Marketing

VASTAR GAS MARKETING

Wascana Energy Marketing inc.

Energy Source Inc.

Noyes, Minnesota

$117 \quad 4.27$

O N/A

$0 \quad$ N/A

VASTAR GAS MARKETING

Wascana Energy Marketing inc.

LG\&E Natural Marketing Inc.

VASTAR GAS MARKETING

Wascana Energy Marketing Inc.

Northem Indiana Public Service

VASTAR GAS MARKETING

Wascana Energy Marketing Inc.

Quick Trade

Noyes, Minnesota

Highgate Spr., VT

CNG Energy

Vermont Gas Systems, Inc.

VERMONT GAS SYSTEMS, INC.

Enron Capital \& Trade Resources Can

Vermont Gas Systems, Inc.

Highgate Spr., VT

$8 \quad 3.38$

$8 \quad 2.34$

134

2.17

150

VERMONT GAS SYSTEMS, INC.

Semco Energy Services, inc.

Vemont Gas Systems, Inc.
Highgate Spr., VT
117

4.27

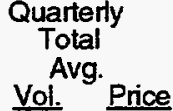

19

$18 \quad 2.84$

$61 \quad 2.04$

$251 \quad 2.25$

$65 \quad 3.84$

$31 \quad 2.88$
- N/A

$10 \quad 2.41$

10

2.41 


\section{SHORT-TERM SALES \\ YEAR: 1997 Quarter: One \\ Estimated Volumes (MMCF) \& Prices (\$ / MMBTU)}

Importer

Seller

Purchaser / End User

VERMONT GAS SYSTEMS, INC.

TransCanada Gas Services Limited

Vermont Gas Systems, Inc.

VERMONT GAS SYSTEMS, INC.

Westcoast Energy Marketing, Ltd.

Vermont Gas Systems, Inc.
January

of

Entry

Highgate Spr., VT
$0 \quad$ N/A

WASCANA ENERGY MARKETING (U.S.) INC.

Wascana Energy Marketing Inc.

Amoco Trading

Noyes, Minnesota

WASCANA ENERGY MARKETING (U.S.) INC.

Wascana Energy Marketing Inc.

Cibola Corporation

Port of Morgan, MT

211

2.27

176

1.96

196

2.32

584

2.19

WASCANA ENERGY MARKETING (U.S.) INC.

Wascana Energy Marketing Inc.

CoEnergy Trading Company

Niagara Falls, NY

- N/A

165

3.78

0

N/A

165

3.78

WASCANA ENERGY MARKETING (U.S.) INC. Wascana Energy Marketing Inc.

Colonial Energy, Inc.

Waddington, NY

$32 \quad 4.03$

$0 \quad N / A$

23

2.26

55

3.28

WASCANA ENERGY MARKETING (U.S.) INC. Wascana Energy Marketing Inc.

EPEC Marketing Company

Grand island, NY

$3 \quad 3.10$

O N/A

O N/A

3

3.10

WASCANA ENERGY MARKETING (U.S.) INC.

Wascana Energy Marketing Inc.

El Paso Gas Marketing

Port of Morgan, MT

152

3.81

O N/A

151

1.31

303

2.56

WASCANA ENERGY MARKETING (U.S.) INC.

Wascana Energy Marketing inc.

Enron Capital \& Trade Resources

Eastport, Idaho

$0 \quad$ N/A

O N/A

302

1.24

302

1.24

WASCANA ENERGY MARKETING (U.S.) INC.

Wascana Energy Marketing Inc.

Enserch Energy Services, lnc.

Grand Island, NY

1524.98

138

3.59

152

2.61

442

3.73

WASCANA ENERGY MARKETING (U.S.) INC.

Wascana Energy Marketing Inc.

Global Petroleum

Grand Island, NY $\begin{array}{llll}0 & \text { N/A } & 3 & 3.26\end{array}$

$2 \quad 2.06$

5

2.73 


\section{SHORT-TERM SALES \\ YEAR: 1997 Quarter: One \\ Estimated Volumes (MMCF) \& Prices (\$/MMBTU)}

Importer

Seller

Purchaser / End User
Point

Entry
January

Vol. Price
February

Vol. Price
Quarterly

Total

Avg.

Vol. Price
WASCANA ENERGY MARKETING (U.S.) INC.

Wascana Energy Marketing Inc.

Howard Energy Company

Grand Island, NY

$45 \quad 3.65$

$5 \quad 3.27$

- N/A

50

3.61

WASCANA ENERGY MARKETING (U.S.) INC.

Wascana Energy Marketing Inc.

Howand Energy Company

Niagara Falls, NY

O N/A

$7 \quad 2.89$

O N/A

$7 \quad 2.89$

WASCANA ENERGY MARKETING (U.S.) INC Wascana Energy Marketing Inc.

Howard Energy Company

Noyes, Minnesota

$20 \quad 2.87$

142

3.11

O N/A

161

3.08

WASCANA ENERGY MARKETING (U.S.) INC.

Wascana Energy Marketing Inc.

Kumball Trading Company, L.L.C.

Noyes, Minnesota

$10 \quad 2.80$

- N/A

- N/A

10

2.80

WASCANA ENERGY MARKETING (U.S.) INC

Wascana Energy Marketing Inc.

Koch Gas Services Company

Noyes, Minnesota

$67 \quad 3.30$

$22 \quad 2.39$

$88 \quad 2.02$

176

2.55

WASCANA ENERGY MARKETING (U.S.) INC.

Wascana Energy Marketing Inc.

Midcon Gas Senvices Corporation

Noyes, Minnesota

$168 \quad 3.20$

$0 \quad$ N/A

$0 \quad$ N/A

168

WASCANA ENERGY MARKETING (U.S.) INC.

Wascana Energy Marketing Inc.

Minnesota Corm Processors

Port of Morgan, MT

$91 \quad 1.78$

163

2.15

182

1.17

436

1.66

WASCANA ENERGY MARKETING (U.S.) INC.

Wascana Energy Marketing Inc.

National Fuel Gas Distribution

Grand Island, NY

$81 \quad 3.89$

15

2.10

- N/A

96

3.61

WASCANA ENERGY MARKETING (U.S.) INC

Wascana Energy Marketing Inc.

National Fuel Gas Distribution

Niagara Falls, NY

$0 \quad$ N/A

$81 \quad 2.43$

- N/A

$81 \quad 2.43$

WASCANA ENERGY MARKETING (U.S.) INC. Wascana Energy Marketing Inc.

National Fuel Resources

Niagara Falls, NY

- N/A

O N/A

$20 \quad 2.08$

20

2.08

WASCANA ENERGY MARKETING (U.S.) INC.

Wascana Energy Marketing Inc.

PanEnergy Trading \& Marketing Svcs.

Eastport, Idaho

$514 \quad 3.99$

462

2.35

207

0.91

1183

2.81 
SHORT-TERM SALES

YEAR: 1997 Quarter: One

Estimated Volumes (MMCF) \& Prices (\$ / MMBTU)
Importer

Seller

Purchaser/End User
Point

of

Entry
Vol. Price
January

Grand Island, NY

Wascana Energy Marketing Inc.

PanEnergy Trading \& Marketing Svcs.

WASCANA ENERGY MARKETING (U.S.) INC.

Wascana Energy Marketing Inc.

PanEnergy Trading \& Marketing Svcs.

Noyes, Minnesota

709

3.88

126

3.20

Niagara Falls, NY

Wascana Energy Marketing Inc.

Penn Union Energy

WASCANA ENERGY MARKETING (U.S.) INC.

Wascana Energy Marketing Inc.

SEMCO Energy Services

Noyes, Minnesota

WASCANA ENERGY MARKETING (U.S.) INC.

Wascana Energy Marketing Inc.

Sierra Pacific Power Company

Eastport, Idaho

34

342

1.60

393

March

Vol. Price

Vol. Price

Quarterty

Total

Avg.

Vol. Price

WASCANA ENERGY MARKETING (U.S.) INC.

Wascana Energy Marketing inc.

Tenaska Marketing Ventures

Noyes, Minnesota

$\begin{array}{llll}0 & \text { N/A } & 158 & 2.88\end{array}$

- N/A

158

2.88

WASCANA ENERGY MARKETING (U.S.) INC.

Wascana Energy Marketing inc.

Tenaska Marketing Ventures

Port of Morgan, MT

297

340

2.51

$231 \quad 1.40$

869

2.66

WASCANA ENERGY MARKETING (U.S.) INC.

Wascana Energy Marketing Inc.

Texaco Natural Gas

Grand Island, NY

122

4.60

110

4.50

$0 \quad$ N/A

232

4.55

WASCANA ENERGY MARKETING (U.S.) INC.

Wascana Energy Marketing Inc.

Texaco Natural Gas

Niagara Falls, NY

O N/A

$\begin{array}{ll}63 & 3.77\end{array}$

- N/A

63

3.77

WASCANA ENERGY MARKETING (U.S.) INC.

Wascana Energy Marketing Inc.

Utilicorp United

Noyes, Minnesota

10

4.67

$0 \quad N / A$

O N/A

10

4.67

WASCANA ENERGY MARKETING (U.S.) INC.

Wascana Energy Marketing Inc.

Vastar Gas

Noyes, Minnesota
162

3.94

144

2.88

159

1.89

465

2.91 
SHORT-TERM SALES

YEAR: 1997 Quarter: One

Estimated Volumes (MMCF) \& Prices (\$ / MMBTU)
Importer

Seller

Purchaser / End User
Point

of

Entry
January
Vol. Price
February
Vol. Price
March

Vol Price
Quarterty

Total Avg. Vol.

WASCANA ENERGY MARKETING (U.S.) INC.

Wascana Energy Marketing Inc.

WPS Energy Services, Inc.

Noyes, Minnesota

$153 \quad 3.92$

- N/A

0 N/A

153

3.92

WASCANA ENERGY MARKETING (U.S.) INC.

Wascana Energy Marketing Inc.

Westcoast Gas Services (U.S.A.) inc

Noyes, Minnesota

$295 \quad 4.06$

$243 \quad 3.19$

O N/A

538

3.66

WASCANA ENERGY MARKETING (U.S.) INC.

Wascana Energy Marketing inc.

Wickford Energy Marketing Inc.

Niagara Falls, NY

o. N/A

$3 \quad 3.31$

O N/A

3

3.31

WASHINGTON WATER POWER COMPANY

Amoco Canada Petroleum Company Ltd.

Washington Water Power Company

Eastport, Idaho

$310 \quad 1.61$

$140 \quad 1.54$

o. N/A

450

1.58

WASHINGTON WATER POWER COMPANY

B.C. Gas Inc.

Washington Water Power Company

Sumas, Washington

$127 \quad 4.16$

- N/A

$0 \quad$ N/A

127

4.16

WASHINGTON WATER POWER COMPANY El Paso Energy Markeing Canada

Washington Water Power Company

Eastport, Idaho

$0 \quad$ N/A

$130 \quad 1.65$

29

1.42

426

1.49

WASHINGTON WATER POWER COMPANY

Enron Capital \& Trade Resources Can

Washington Water Power Company

Eastport, Idaho

$304 \quad 1.66$

$275 \quad 2.11$

303

1.30

881

1.68

WASHINGTON WATER POWER COMPANY

PanCanadian Petroleum Ltd.

Washington Water Power Company

Eastport, Idaho

$152 \quad 1.02$

$137 \quad 1.02$

$152 \quad 1.02$

441

1.02

WASHINGTON WATER POWER COMPANY

PanEnergy Marketing Canada

Washington Water Power Company

Eastport, Idaho

$0 \quad$ N/A

o. N/A

152

1.21

152

1.21

WASHINGTON WATER POWER COMPANY

Petro-Canada Hydrocarbons Inc.

Washington Water Power Company

Eastport, Idaho

$455 \quad 1.76$

$275 \quad 1.93$

O N/A

730

1.82

WASHINGTON WATER POWER COMPANY

Westcoast Gas Services Inc.

Washington Water Power Company

Eastport, Idaho

152

2.10

N/A

0

N/A

152

2.10 
SHORT-TERM SALES

YEAR: 1997 Quarter: One

Estimated Volumes (MMCF) \& Prices (\$ / MMBTU)

Importer

Seller

Purchaser / End User

Point

Entry

WESTCOAST GAS SERVICES (AMERICA) INC.

Westcoast Gas Services Inc.

Sierra Pacific Power Company

Eastport, Idaho

WESTCOAST GAS SERVICES (AMERICA) INC.

Westcoast Gas Services Inc.

Various WA State Markets

Sumas, Washington

Grand Island, NY

Westcoast Gas Services (U.S.A.), In

Eastem Energy Marketing

WESTCOAST GAS SERVICES (U.S.A.), INC.

Westcoast Gas Senvices (U.S.A.), in

Enserco Energy, Inc.

Grand Island, NY

Massena, New York

16

1.17

14

1.17

17

1.17

47

1.17

WESTCOAST GAS SERVICES INC.

Westcoast Gas Services Inc.

Ranck Oil Company

Port of Morgan, MT

$0 \quad$ N/A

O N/A

$\begin{array}{ll}9 & 1.27\end{array}$

9

1.27

WESTCOAST GAS SERVICES INC.

Westcoast Gas Services Inc.

St. Lawrence University

Massena, New York

$11 \quad 1.12$

10

1.12

12

1.12

33

1.12

WESTCOAST GAS SERVICES INC.

Westcoast Gas Services Inc.

Westcoast Gas Svcs. (America), Inc.

Eastport, Idaho

$83 \quad 1.28$

128

2.26

$91 \quad 1.36$

302

1.72

WESTCOAST GAS SERVICES INC.

Westcoast Gas Services inc.

Westcoast Gas Svcs. (America), Inc.

Port of Morgan, MT

133

3.86

1336

2.53

1437

1.33

4105

2.54

WESTCOAST GAS SERVICES INC.

Westcoast Gas Services Inc.

Westcoast Gas Svcs. (America), Inc.
Sumas, Washington

337

4.15

370

2.37

469

1.05

2.35 
AIG TRADING CORPORATION

AMOCO ENERGY TRADING CORPORATION ANADARKO TRADING COMPANY

BC GAS UTILITY LTD.

BURLINGTON RESOURCES TRADING INC.

CENTRA GAS ONTARIO INC.

CHANNEL GAS MARKETING COMPANY

CHEVRON U.S.A. INC.

COASTAL GAS MARKETING COMPANY

COENERGY TRADING COMPANY

CONOCO INC.

CONSUMERS' GAS COMPANY LIMITED

CORAL ENERGY RESOURCES, L.P.

COWEST ENERGY

EL PASO ENERGY MARKETING COMPANY

EL PASO GAS MARKETING COMPANY

ENRON CAPITAL \& TRADE RESOURCES CORP.

GULF ENERGY MARKETING COMPANY

HOWARD ENERGY COMPANY, INC.

KN MARKETING, L.P.

LG\&E NATURAL MARKETING INC.

LOUIS DREYFUS ENERGY CORPORATION

MIDCON GAS SERVICES CORPORATION

MIDCON TEXAS GAS SERVICES CORPORATION

MOBIL NATURAL GAS INC.

NATIONAL ENERGY \& TRADE, L.L.C.

NATURAL GAS CLEARINGHOUSE

NORAM ENERGY SERVICES, INC.

NORTH AMERICAN RESOURCES COMPANY

ONYX GAS MARKETING COMPANY, L.C.

PANENERGY GAS SERVICES, INC.

PANENERGY TRADING AND MARKET SERVICES L.L.C.

PHIBRO INC.

PHILLIPS ALASKAMMARATHON OIL

QUESTAR ENERGY TRADING COMPANY

ST. CLAIR PIPELINES LTD.

TARPON GAS MARKETING LTD.

TENASKA MARKETING VENTURES

TENNECO GAS MARKETING COMPANY

TEXACO NATURAL GAS INC.

TRANSCANADA GAS SERVICES INC.

TRISTAR GAS MARKETING COMPANY

UNION GAS LIMITED

UNION PACIFIC FUELS, INC.

VALERO GAS MARKETING, L.P.

VALERO INDUSTRIAL GAS, L.P.

WASCANA ENERGY MARKETING (U.S.) INC.

WEST TEXAS GAS, INC.

WESTCOAST GAS SERVICES (U.S.A.), INC.

WESTERN GAS RESOURCES, INC.

\begin{tabular}{|c|c|c|c|c|}
\hline $\begin{array}{c}1996 \\
\text { Jan,-Mar. }\end{array}$ & $\begin{array}{c}1996 \\
\text { Apr.Jun. }\end{array}$ & $\begin{array}{r}1996 \\
\text { Jul.-Sep. }\end{array}$ & $\begin{array}{c}1996 \\
\text { Oct.-Dec. }\end{array}$ & $\begin{array}{c}1997 \\
\text { Jan.-Mar. }\end{array}$ \\
\hline 1569 & 1509 & 908 & 424 & 1900 \\
\hline 2085 & 1323 & 1860 & 915 & 67 \\
\hline 0 & 0 & 11 & 134 & 154 \\
\hline 434 & 0 & 0 & 999 & 0 \\
\hline 0 & 111 & 0 & 65 & 54 \\
\hline 1741 & 0 & 0 & 1218 & 1729 \\
\hline 58 & 317 & 222 & 0 & 0 \\
\hline 266 & 68 & 252 & 0 & 0 \\
\hline 0 & 0 & 2366 & 685 & 0 \\
\hline 4370 & 345 & 790 & 3970 & 3857 \\
\hline 1292 & 492 & 1208 & 760 & 1957 \\
\hline 1691 & 520 & 0 & 1034 & 2012 \\
\hline 0 & 0 & 995 & 0 & 0 \\
\hline 69 & 0 & 0 & 315 & 33 \\
\hline 0 & 219 & 0 & 0 & 320 \\
\hline 0 & 0 & 1189 & 0 & 0 \\
\hline 121 & 1325 & 331 & 100 & 399 \\
\hline 60 & 270 & 0 & 0 & 0 \\
\hline 530 & 50 & 0 & 0 & 185 \\
\hline 871 & 0 & 1011 & 547 & 145 \\
\hline 0 & 264 & 31 & 0 & 0 \\
\hline 0 & 0 & 0 & 16 & 233 \\
\hline 0 & 0 & 250 & 302 & 105 \\
\hline 0 & 0 & 20 & 0 & 0 \\
\hline 0 & 0 & 486 & 0 & 0 \\
\hline 0 & 0 & 53 & 0 & 0 \\
\hline 107 & 251 & 448 & 518 & 442 \\
\hline 2506 & 1114 & 636 & 338 & 460 \\
\hline 0 & 0 & 0 & 91 & 171 \\
\hline 0 & 2 & 0 & 0 & 0 \\
\hline 0 & 160 & 861 & 0 & 0 \\
\hline 0 & 0 & 0 & 0 & 19 \\
\hline 0 & 0 & 157 & 0 & 0 \\
\hline 0 & 0 & 0 & 0 & 104 \\
\hline 18 & 0 & 0 & 0 & 0 \\
\hline 583 & 0 & 0 & 0 & 0 \\
\hline 4 & 0 & 0 & 0 & 0 \\
\hline 71 & 0 & 188 & 470 & 1422 \\
\hline 176 & 0 & 0 & 0 & 0 \\
\hline 0 & 0 & 0 & 952 & 48 \\
\hline 2431 & 3005 & 1056 & 0 & 0 \\
\hline 154 & 643 & 26 & 59 & 683 \\
\hline 6521 & 6605 & 6617 & 7507 & 5998 \\
\hline 0 & 95 & 30 & 0 & 0 \\
\hline 0 & 0 & 0 & 0 & 11 \\
\hline 305 & 491 & 1814 & 0 & 0 \\
\hline 175 & 0 & 0 & 552 & 1857 \\
\hline 0 & 56 & 162 & 288 & 358 \\
\hline 579 & 0 & 0 & 0 & 0 \\
\hline 0 & 0 & 0 & 0 & 110 \\
\hline
\end{tabular}




\section{SHORT-TERM EXPORTERS}

Estimated Volumes (MMCF)

1996

Jan.-Mar.

0

28791
1996

Apr.-Jun.

272

19506
1996

Jul.-Sep.

488

24467
1996

Oct.-Dec.

193

$\underline{22452}$
1997 Jan.-Mar.

184

25015 


\section{PURCHASERS OF SHORT-TERM EXPORTS \\ Estimated Volumes Exported (MMCF)}

\begin{tabular}{|c|c|c|c|c|}
\hline $\begin{array}{c}1996 \\
\text { Jan.-Mar. }\end{array}$ & $\begin{array}{c}1996 \\
\text { Apr.-Jun. }\end{array}$ & $\begin{array}{c}1996 \\
\text { Jul.-Sep. }\end{array}$ & $\begin{array}{c}1996 \\
\text { Oct.-Dec. }\end{array}$ & $\begin{array}{c}1997 \\
\text { Jan.-Mar. }\end{array}$ \\
\hline 1225 & 0 & 0 & 0 & 313 \\
\hline 0 & 0 & 0 & 600 & 0 \\
\hline 452 & 0 & 0 & 999 & 0 \\
\hline 1997 & 0 & 0 & 450 & 15 \\
\hline 0 & 0 & 0 & 0 & 9 \\
\hline 1691 & 865 & 0 & 0 & 0 \\
\hline 220 & 0 & 0 & 0 & 33 \\
\hline 18 & 0 & 0 & 0 & 0 \\
\hline 0 & 0 & 0 & 0 & 459 \\
\hline 0 & 0 & 0 & 94 & 0 \\
\hline 176 & 0 & 0 & 0 & 475 \\
\hline 117 & 0 & 0 & 0 & 0 \\
\hline 0 & 0 & 0 & 1620 & 1822 \\
\hline 234 & 0 & 0 & 0 & 280 \\
\hline 0 & 290 & 31 & 0 & 0 \\
\hline 0 & 14 & 0 & 300 & 0 \\
\hline C. & 0 & 0 & 15 & 0 \\
\hline 6467 & 7309 & 14627 & 5436 & 5380 \\
\hline 145 & 0 & 0 & 0 & 0 \\
\hline 583 & 0 & 0 & 0 & 0 \\
\hline 0 & 0 & 306 & 0 & 0 \\
\hline 4 & 0 & 0 & 0 & 0 \\
\hline 0 & 0 & 0 & 0 & 104 \\
\hline 2431 & 3005 & 1056 & 0 & 0 \\
\hline 10463 & 8014 & 790 & 800 & 152 \\
\hline 0 & 0 & 0 & 200 & 0 \\
\hline 0 & 0 & 0 & 91 & 171 \\
\hline 1812 & 0 & 7656 & 11270 & 13166 \\
\hline 175 & 0 & 0 & 577 & 2009 \\
\hline 579 & 0 & 0 & 0 & 611 \\
\hline 0 & 0 & 0 & 0 & 15 \\
\hline 28791 & 19506 & 24467 & 22452 & 25015 \\
\hline
\end{tabular}

AlG Trading Corporation

Alberta Energy Company

BC Gas Inc.

Centra Gas Ontario Inc.

Cibola Canada

Consumers' Gas Company Limited

Cowest Energy

Dawn Storage

Energy Source Inc.

Enron Capital \& Trade Resources Canada

Enron Gas Marketing Canada, Inc.

Enserch Energy Services, inc.

Gaz Metropolitain

Howard Energy Company

LG\&E Natural Marketing Canada Inc.

National Steel Corporation

PanEnergy Trading \& Marketing Svcs., Inc., L.L.C. 0

PEMEX

Phibro Oil \& Gas Inc.

St. Clair Pipelines Ltd.

Storage in Ontario

Tarpon Gas Marketing Ltd.

Tokyo Gas Co.JTokyo Electric Power Co.

TransCanada Gas Services Limited

Union Gas Limited

Utilicorp United

Various Alberta Customers

Various Ontario Customers

Wascana Energy Marketing

Westcoast Gas Services Inc.

Wickford Energy Marketing Inc.

Totals
19506 
SHORT-TERM SALES

YEAR: 1997 Quarter: One

Estimated Volumes (MMCF) \& Prices (\$ / MMBTU)
Exporter

Seller

Purchaser / End User

AIG TRADING CORPORATION

AIG Trading Corporation

Various Ontario Customers

AIG TRADING CORPORATION

AIG Trading Corporation

Various Ontario Customers

AIG TRADING CORPORATION

AIG Trading Corporation

Various Ontario Customers

St. Clair, Michigan

Hidalgo, Texas

Anadarko Trading Company

PEMEX

Hidalgo, Texas

$7 \quad 2.86$

$58 \quad 2.36$

21.68

$67 \quad 2.40$

ANADARKO TRADING COMPANY

Anadarko Trading Company

Union Gas Limited

St. Clair, Michigan

BURLINGTON RESOURCES TRADING INC.

Burlington Resources Trading inc.

PEMEX

El Paso, Texas

$54 \quad 4.09$

O N/A

o N/A

54

4.09

CENTRA GAS ONTARIO INC.

Centra Gas Ontario Inc.

Various Ontario Customers

Detroit, Michigan

559

3.95

549

2.75

621

1.83

1729

2.81

COENERGY TRADING COMPANY

CoEnergy Trading Company

AIG Trading Corporation

St. Clair, Michigan

COENERGY TRADING COMPANY

CoEnergy Trading Company

Centra Gas Ontario Inc.

St. Clair, Michigan

- N/A

O N/A

15

2.14

15

2.14

COENERGY TRADING COMPANY

CoEnergy Trading Company

Cibola Canada

St. Clair, Michigan
- N/A

- N/A

9

2.14

2.14 
SHORT-TERM SALES

YEAR: 1997 Quarter: One

Estimated Volumes (MMCF) \& Prices (\$/MMBTU)
Exporter

Seller

Purchaser / End User

COENERGY TRADING COMPANY CoEnergy Trading Company

Energy Source inc.

COENERGY TRADING COMPANY

CoEnergy Trading Company

Enron Gas Marketing Canada, Inc.

COENERGY TRADING COMPANY

CoEnergy Trading Company

Gaz Metropolitain

St. Clair, Michigan

January

of
Exit

St. Clair, Michigan

St. Clair, Michigan

$108 \quad 3.70$

o N/A

367

March

Vol. Price

February

Vol. Price

Vol. Price

Quarterty

Total

Avg.

Vol.

$0 \quad$ N/A

$0 \quad$ N/A

459

2.14

459

2.14

COENERGY TRADING COMPANY

CoEnergy Trading Company

Wascana Energy Marketing

COENERGY TRADING COMPANY

CoEnergy Trading Company

Westcoast Gas Services inc.

COENERGY TRADING COMPANY

LG\&E Natural Marketing Canada Inc.

Westcoast Gas Services Inc.

St. Clair, Michigan

$0 \quad$ N/A

O N/A

306

2.14

306

2.14

CONOCO INC.

Conoco Inc.

PEMEX

Douglas, Arizona

4.04

759

2.37

778

1.47

1957

2.37

CONSUMERS' GAS COMPANY LIMITED American Hunter Exploration Ltd.

Various Ontario Customers

Marysville, Michigan

- N/A

$149 \quad 2.88$

166

1.55

315

2.18

CONSUMERS' GAS COMPANY LIMITED Aquila Energy Marketing

Various Ontario Customers

Marysville, Michigan

- N/A

149

2.88

165

1.66

314

2.24

CONSUMERS' GAS COMPANY LIMITED

Dreyfus

Vanous Ontario Customers

Marysville, Michigan

$325 \quad 4.41$

o N/A

$0 \quad$ N/A

325

4.41

CONSUMERS' GAS COMPANY LIMITED Kimball Energy Corporation

Various Ontario Customers
Marysville, Michigan
313

1.87

313

1.87 
SHORT-TERM SALES

YEAR: 1997 Quarter: One

Estimated Volumes (MMCF) \& Prices (\$/MMBTU)
Exporter

Seller

Purchaser / End User

CONSUMERS' GAS COMPANY LIMITED

Michigan Consolidated

Various Ontario Customers

Point

of

Exit

St. Clair, Michigan

Detroit, Michigan

St. Clair, Michigan

COWEST ENERGY

Cowest Energy

Cowest Energy

CONSUMERS' GAS COMPANY LIMITED

Proliance

Various Ontario Customers

$0 \quad$ N/A

O N/A

$181 \quad 2.08$

181

2.08

EL PASO ENERGY MARKETING COMPANY

El Paso Energy Marketing Company

PEMEX

El Paso, Texas

19

3.28

300

2.52

0

$0 \quad$ N/A

33

3.46

ENRON CAPITAL \& TRADE RESOURCES CORP.

Enron Capital \& Trade Resources

PEMEX

El Paso, Texas

Marysville, Michigan

O N/A$$
\text { N/A }
$$$$
185 \quad 2.23
$$

KN MARKETING, L.P.

KN Marketing, L.P.

PEMEX

El Paso, Texas

82

3.83

$31 \quad 1.84$

$32 \quad 1.88$

145

2.97

LOUIS DREYFUS ENERGY CORPORATION

Various Suppliers

PEMEX

El Paso, Texas

66

3.59

$142 \quad 1.91$

$25 \quad 1.80$

233

2.38

MIDCON GAS SERVICES CORPORATION

Midcon Gas Services Corporation

Various Ontario Customers

St. Clair, Michigan

- N/A

- N/A

105

2.20

105

2.20

NATURAL GAS CLEARINGHOUSE

Natural Gas Clearinghouse

PEMEX

El Paso, Texas

$372 \quad 4.09$

$60 \quad 1.75$

$10 \quad 1.70$

442

3.72

NORAM ENERGY SERVICES, INC.

Noram Energy Services

PEMEX

El Paso, Texas

199

4.03

$33 \quad 1.71$

228

1.52

460

2.62 


\section{SHORT-TERM SALES \\ YEAR: 1997 Quarter: One \\ Estimated Volumes (MMCF) \& Prices (\$ / MMBTU)}

$\begin{array}{ll}\text { Exporter } & \text { Point } \\ \text { Seller } & \text { of } \\ \text { Purchaser / End User } & \text { Exit }\end{array}$

NORTH AMERICAN RESOURCES COMPANY

North American Resources Company

Various Alberta Customers

Babb, Montana

107

January

Vol. Price

February

Vol. Price

March
Vol. Price

Quarterty

Total

Avg

Vol.

Price

PANENERGY TRADING AND MARKET SERVICES L.L.C.

PanEnergy Trading

PEMEX

Hidalgo, Texas

Kenai, Alaska

Kenai, Alaska

Detroit, Michigan

TENASKA MARKETING VENTURES

Tenaska Marketing Ventures

Various Ontario Customers

TENASKA MARKETING VENTURES

Tenaska Marketing Ventures

Various Ontario Customers

TEXACO NATURAL GAS INC.

Texaco Natural Gas Inc.

PEMEX

El Paso, Texas

$2 \quad 3.76$

$5 \quad 1.77$

$41 \quad 1.65$

48

1.75

TRISTAR GAS MARKETING COMPANY Tristar Gas Marketing Company PEMEX

El Paso, Texas

395

4.09

$36 \quad 1.82$

252

1.46

683

3.00

UNION GAS LIMITED

Amoco Energy Trading Corporation

Various Ontano Customers

Detroit, Michigan

$309 \quad 4.01$

$0 \quad N / A$

O N/A

309

4.01

UNION GAS LIMITED

Anadarko Trading Company

Various Ontario Customers

Detroit, Michigan

3.76

$489 \quad 2.82$

696

1.82

1417

2.48

UNION GAS LIMITED

BNG Inc.

Various Ontario Customers

Detroit, Michigan
$309 \quad 4.01$

$0 \quad$ N/A

O N/A

309

4.01 


\section{SHORT-TERM SALES \\ YEAR: 1997 Quarter: One \\ Estimated Volumes (MMCF) \& Prices (\$ / MMBTU)}

Exporter
Seller

Purchaser / End User

UNION GAS LIMITED

Coastal Gas Marketing Company

Various Ontario Customers

Point
of

Exit

Detroit, Michigan

St. Clair, Michigan

Detroit, Michigan

Detroit, Michigan

Detroit, Michigan

$309 \quad 3.96$

O N/A

- N/A

309

3.96

VALERO GAS MARKETING, L.P.

Valero Gas Marketing, L.P.

PEMEX

El Paso, Texas

D N/A

$8 \quad 1.79$

$2 \quad 1.85$

11

1.80

WASCANA ENERGY MARKETING (U.S.) INC.

Comsatec

Wascana Energy Marketing

St. Clair, Michigan

o N/A

$84 \quad 4.06$

O N/A

84

4.06

WASCANA ENERGY MARKETING (U.S.) INC.

Phibro Division of Salomon Inc.

Wascana Energy Marketing

St. Clair, Michigan

$275 \quad 4.47$

$275 \quad 3.40$

$948 \quad 2.06$

1498

2.75

WASCANA ENERGY MARKETING (U.S.) INC.

SEMCO

Wascana Energy Marketing

St. Clair, Michigan

o N/A

$275 \quad 3.38$

- N/A

275

3.38

WEST TEXAS GAS, INC.

West Texas Gas, Inc.

PEMEX

Eagle Pass, Texas

$144 \quad 4.01$

121

2.89

$93 \quad 1.85$

358

3.07

WESTERN GAS RESOURCES, INC.

Western Gas Resources

Howard Energy Company

Detroit, Michigan
- N/A

- N/A

$95 \quad 2.21$

95

2.21 
SHORT-TERM SALES

YEAR: 1997 Quarter: One

Estimated Volumes (MMCF) \& Prices (\$ / MMBTU)

\section{Exporter \\ Seller}

Purchaser / End User

WESTERN GAS RESOURCES, INC.

Western Gas Resources

Wickford Energy Marketing Inc.

Detroit, Michigan

\section{Point \\ Of}

WILLIAMS ENERGY SERVICES COMPANY Williams Energy Services Company

PEMEX
Douglas, Arizona
January

Vol. Price

February

Vol. Price

March

Vol. Price

$0 \quad$ N/A

o N/A

15

2.23

15

2.23
$136 \quad 4.06$

$46 \quad 1.79$

$2 \quad 1.78$

184

3.48 